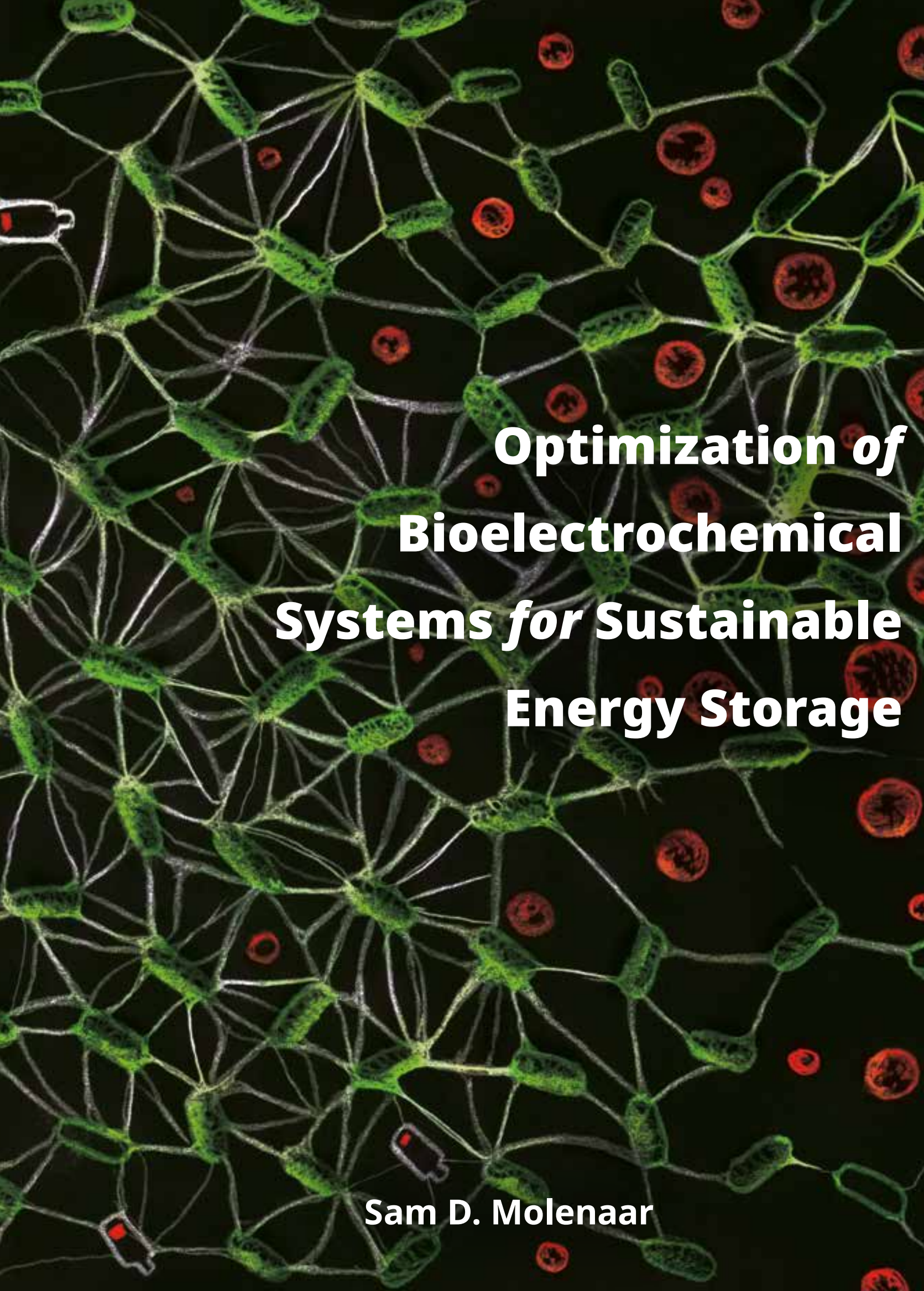





\section{Optimization of Bioelectrochemical Systems for Sustainable Energy Storage}

Sam D. Molenaar 


\section{Thesis committee}

\section{Promotor}

Prof. Dr C.J.N. Buisman

Professor of Biological Recovery and Re-use Technology

Wageningen University \& Research

\section{Co-promotors}

Dr A. ter Heijne

Assistant professor, Sub-department of Environmental Technology

Wageningen University \& Research

Dr T.H.J.A. Sleutels

Researcher in Bioprocess Technology \& (bio-)Electrochemistry

Wetsus, Leeuwarden

\section{Other members}

Prof. Dr K.J. Keesman, Wageningen University \& Research

Prof. Dr K. Rabaey, Ghent University, Belgium

Prof. Dr U. Schröder, Technische Universität Braunschweig, Germany

Dr A. Deeke, Waterschap de Dommel, Boxtel

This research was conducted under the auspices of the Graduate School for Socio-Economic and Natural Sciences of the Environment (SENSE) 


\title{
Optimization of Bioelectrochemical Systems for Sustainable Energy Storage
}

\author{
Sam D. Molenaar
}

Thesis

submitted in fulfilment of the requirements for the degree of doctor

at Wageningen University

by the authority of the Rector Magnificus,

Prof. Dr A.P.J. Mol,

in the presence of the

Thesis Committee appointed by the Academic Board

to be defended in public

on Wednesday 7 November 2018

at 1:30 p.m. in the Aula. 
Optimization of Bioelectrochemical Systems for Sustainable Energy Storage Sam D. Molenaar

188 pages.

PhD thesis, Wageningen University, Wageningen, The Netherlands (2018)

With references, with summary in English

ISBN: 978-94-6343-518-5

DOI: https://doi.org/10.18174/461159 




\section{FOREWORD}

A transition to renewable energy is inevitable. The negative environmental aspects of the use of fossil energy resources and the fact that (known) fossil fuel reserves are finite and depletion is thought to occur in a matter of decades to centuries, force us to use renewable resources like sun and wind. An imminent disadvantage of using these low power density resources is the almost unimaginable scale of harvesting infrastructure that would be required to fully replace the current energy demands met by fossil fuel use, the production and operation of which most likely will cause severe (additional) environmental side-effects. In this sense, the transition to renewable energy cannot be separated from the need for a broader transition to a more sustainable macroeconomic system, in which long term effects of human activities are more adequately addressed and acted upon. This vision has been reinforced by the club of Rome for decades ${ }^{[1]}$, and has very recently been invigorated by the World Scientists' warning to humanity ${ }^{[2]}$. These publications have received overwhelming support by the international scientific community including most living Nobel laureates, and stress the need for urgent and abrupt changes in multiple domains, as the following citation from the latter publication shows:

"The scientists pleaded that we stabilize the human population, describing how our large numbers - swelled by another 2 billion people since 1992, a 35 percent increaseexert stresses on Earth that can overwhelm other efforts to realize a sustainable future. They implored that we cut greenhouse gas (GHG) emissions and phase out fossil fuels, reduce deforestation, and reverse the trend of collapsing biodiversity"

When aiming for a high quality of life for all human beings living now and for future generations, a concerted effort in both technological and societal domains is required. For a more quantitative perspective on how humanity could possibly maneuver itself within safe planetary boundaries, the reader is referred to the recent publication by O'Neill et $\mathrm{al}^{[3]}$. Provided this context, the remainder of this thesis has to be regarded with the clear notion that the transition towards renewable energy sources is thus only a sub-solution to the broader problem of self-caused existential threats humanity is currently facing.

(1) Meadows, D.; Randers, J.; Meadows, D. The Limits to Growth: The 30-Year Update; Chelsea Green Publishing, 2004.

(2) Ripple, W.J.; Wolf, C.; Galetti, M.; Newsome, T. M.; Alamgir, M.; Crist, E.; Mahmoud, M. I.; Laurance, W. F.; Galetti, M.; Alamgir, M.; et al. World Scientists' Warning to Humanity: A Second Notice. BioScience 2017, 12, 1026-1028 DOI: $10.1093 /$ biosci/bix125

(3) O'Neill, D. W.; Fanning, A. L.; Lamb, W. F.; Steinberger, J. K. A good life for all within planetary boundaries. Nat. Sustain. 2018, 1 (2), 88-95 DOI: 10.1038/s41893-018-0021-4. 


\section{TABLE OF CONTENTS}

Foreword

Table of contents

1. General Introduction

1.1. Sustainable energy storage technologies for the renewable energy $\quad \begin{aligned} & 1 \\ & 2\end{aligned}$ transition

1.2. A short history of BES

1.3. What makes a good battery?

1.3.1. What will the mismatch look like?

1.3.2. The primary criteria for mitigation of mismatch: capacity and 5 power

1.3.3. A more in-depth analysis of energy storage performance indicators

1.4. MRB fundamentals

1.4.1. Main factors affecting MRB performance

1.5. Research objective and thesis outline

22

1.6. References

2. The Microbial Rechargeable Battery: Energy Storage and Recovery through Acetate

2.1. Introduction

2.2. Materials and methods

2.2.1. System design

2.2.2. Media and microbial inoculum 29

2.2.3. Reactor start-up and operation 30

2.2.4. Chemical analyses and performance calculations 30

2.3. Results and discussion 31

2.4. Acknowledgements 32

2.5. References 36

2.6. Appendices $\quad 37$

2.6. Appendices 38

3. Low Substrate Loading Limits Methanogenesis and Leads to High Coulombic Efficiency in Bioelectrochemical Systems

3.1. Introduction

3.2. Main factors leading to CE losses

3.3. Substrate loading rate as a control parameter for high $\mathrm{CE}$

3.4. Anode overpotential as a control parameter for high Coulombic Efficiency 
3.5. Reaction kinetics and growth parameters 50

3.6. Anode surface area is key to achieve high rates at low substrate 51 Concentrations

3.7. Conclusions 53

3.8. Acknowledgements 53

3.9. References 54

4. Competition between Methanogens and Acetogens in Biocathodes: 57 a Comparison between Potentiostatic and Galvanostatic Control

4.1. Introduction 58

4.2. Materials and methods 61

4.2.1. System design and reactor assembly 61

4.2.2. Electrolyte composition 61

4.2.3. Inoculum 62

4.2.4. Hydraulic and electrochemical operational conditions 62

4.2.5. Analytical techniques 63

4.2.6. Performance calculations 63

4.3. Results 64

4.3.1. Potential controlled experiments 64

4.3.2. Current controlled experiments $\quad 65$

4.4. Discussion 69

4.4.1. Current density as control parameter for MES selectivity towards 70 acetate

4.4.2. Considerations on the effect of current density on overall reactor 72 performance

4.4.3. Other factors affecting (Monod) growth characteristics of $\quad 73$ methanogens and acetogens

4.5. Conclusions 74

4.6. Acknowledgements $\quad 75$

4.7. References 76

5. In situ Biofilm Quantification in Bioelectrochemical Systems 79 using Optical Coherence Tomography

5.1. Introduction 80

5.2. Experimental procedures 82

5.2.1. Reactor setup 82

5.2.2. Reactor inoculation and operation 83

5.2.3. Experimental design $\quad 83$

5.2.4. OCT acquisition and image processing 84

$\begin{array}{ll}\text { 5.2.5. Chemical analyses } & 87\end{array}$

5.2.6. Calculations and conversions used to obtain biomass growth yield 87

5.3. Results and discussion $\quad 88$ 
5.3.1. OCT analysis allows for accurate and precise in situ biomass quantification

5.3.2. Determination of biomass growth yield using a combination 90 of OCT and chronoamperometry

5.3.3. Continuous measurement confirms application of OCT for non-invasive assessment of biofilm growth and activity

5.4. Conclusions

5.5. Acknowledgements 94

5.6. References 95

5.7. Appendices 98

$\begin{array}{ll}\text { 5.7.1. OCT acquisition settings } & 98\end{array}$

5.7.2. Matlab script for OCT image processing 98

6. Comparison of Two Sustainable Counter Electrodes for Energy 101 Storage in the Microbial Rechargeable Battery

$\begin{array}{ll}\text { 6.1. Introduction } & 102\end{array}$

6.2. Materials and methods 103

$\begin{array}{ll}\text { 6.2.1. General MRB design } & 103\end{array}$

6.2.2. Capacitive counter electrode $\quad 105$

6.2.3. Oxygen/water counter electrode 106

$\begin{array}{ll}\text { 6.2.4. Media composition } & 106\end{array}$

$\begin{array}{ll}\text { 6.2.5. MRB inoculum } & 106\end{array}$

6.2.6. MRB start-up and operation 107

6.2.7. Microbial community analysis 108

$\begin{array}{ll}\text { 6.2.8. Chemical analysis and calculations } & 108\end{array}$

$\begin{array}{ll}\text { 6.3. Results and Discussion } & 109\end{array}$

6.3.1. Across-cycle: Coulombic and energy efficiencies for both $\quad 109$ counter electrodes

6.3.2. Within-cycle: electrochemical charging and discharging 112 dynamics

$\begin{array}{ll}\text { 6.3.3. Microbial community diversity } & 116\end{array}$

6.3.4. Comparison of the counter electrodes tested so far: 117 Implications and future perspective

$\begin{array}{ll}\text { 6.4. Acknowledgements } & 120\end{array}$

6.5. References 121

6.6. Appendices 124

6.6.1. Characterization of the capacitive electrode 124

6.6.2. DNA extraction methods and 16S rRNA sequencing 127

6.6.3. Duplicate plots for Coulombic and energy efficiency 128

$\begin{array}{ll}\text { 6.6.4. } \mathrm{O}_{2} / \mathrm{H}_{2} \mathrm{O} \text { counter electrode characterization } & 129\end{array}$ 
7. General Discussion 131

$\begin{array}{ll}\text { 7.1. MRB: current status } & 132\end{array}$

7.1.1. Charge density of the bidirectional carbon dioxide/acetate 133 bioelectrode

7.1.2. Reaction kinetics of the bidirectional carbon dioxide/acetate 135 bioelectrode

7.1.3. Coulombic efficiencies of the bidirectional carbon dioxide/ 138 acetate bioelectrode

7.1.4. Long term stability of the bidirectional carbon dioxide/acetate 139 bioelectrode

7.1.5. Sustainability of the bidirectional carbon dioxide/acetate 141 bioelectrode

7.2. MRB integrated design considerations

7.3. Future outlook of the bidirectional carbon dioxide/acetate 147 bioelectrode

7.4. The position of energy storage as solution of the energy mismatch, 149 and the need for grassroots implementation

7.5. References

7.6. Appendix: practical exploration of choices in mass transfer 155 characteristics by cell design 


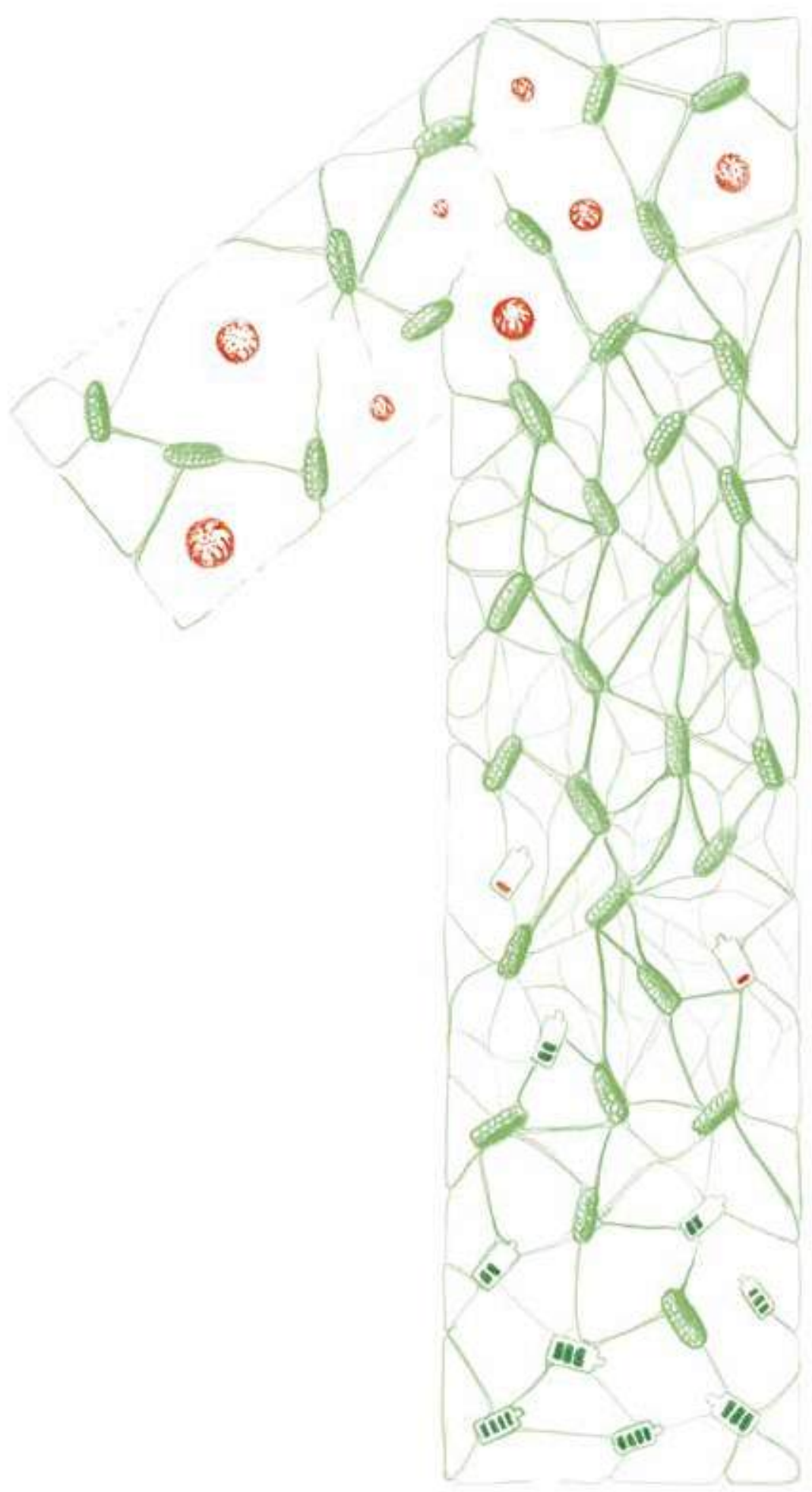




\section{GENERAL INTRODUCTION}

This chapter introduces the reader to the concept of bioelectrochemical systems by providing a short history of their development and a basic technical background on their workings. It contains an analysis regarding the (future) need for sustainable energy storage technologies, allowing the work presented in this thesis to be put in both a technological and societal broader perspective. Furthermore, the aim of the thesis and its outline are described. 


\subsection{SUSTAINABLE ENERGY STORAGE TECHNOLOGIES FOR THE RENEWABLE ENERGY TRANSITION}

A smooth and complete transition towards renewable energy (often referred to as deep-decarbonisation) is challenged by the variability of generation of power from renewable resources, which cannot by themselves function as baseload generation. Under business-as-usual scenarios the integration of renewables beyond $30 \%$ of total grid power generation is generally expected to require additional measures to guarantee baseload providence ${ }^{[1]}$. To certain extent, the future demand for baseload generation will depend on grid flexibility, potentially greatly extending demand-response by creating "smart-grids", and natural balancing arising from harvesting renewable energy sources on a larger spatial scale $^{[2]}$. In addition to this, Energy Storage Technologies (ESTs) which store variable generated renewable energy, could effectively replace the current fossil fuel driven baseload generation. ESTs are therefore widely seen as potential key players in achieving deep decarbonization ${ }^{[3]}$. However, implying such an intensified future role for energy storage technologies, it becomes increasingly important for the production, operation and recommissioning of these storage facilities not to be in conflict with the sustainability endeavors which their intended use is part of. It is therefore essential to include extensive life-cycle assessments (LCA) into the design of these ESTs of the future ${ }^{[4]}$. An LCA assesses "environmental impacts associated with all the stages of a product's life from raw material extraction through materials processing, manufacture, distribution, use, repair and maintenance, and disposal or recycling"[5]. By this definition, conventional electrochemical ESTs may be generally regarded as problematic, as they often rely on substantial inputs of scarce and/or toxic (metallic) compounds, resulting in an overall large environmental impact throughout their life-cycle.

The strong need for energy storage having low environmental impact is what brings relevance to the concept of a Microbial Rechargeable Battery (MRB). In a MRB, electrochemical conversions are catalysed by means of living microbial cells. Together with other technologies exploiting this principle, it is defined as a Bioelectrochemical System (BES).

In BESs, microorganisms are able to sustain their growth and activity by using electrodes either as the electron acceptor or donor required for driving their cellular respiration. Microorganisms are able to do so by exploiting various mechanisms, transferring electrons between an electrode and the microbial cell. This way, a wide variety of complex electron transfer chains (ETCs) naturally present in bacterial communities, carrying out the redox reactions occurring in bacterial catabolism, from oxidation of a wide range of carbohydrates, ammonium 
and fatty acids to the reduction of nitrate, oxygen, carbon dioxide or sulphate, may be ported to the artificial environment of a man-made electrochemical cell[6].

\subsection{A SHORT HISTORY OF BES}

The first account on the ability of microbes interfacing metabolic activities with electrodes is generally ascribed to Potter, who did so as early as $1911^{[7]}$. Initially not leading to substantial follow-up, research in BESs received growing interest during a rediscovery in the late 1950's, as a spin-off from the fast developments occurring at that time in the field of fuel cells ${ }^{[8]}$. Although some interesting fundamental properties (mainly on the varying requirements of electron-mediating compounds) were discovered at this stage, the field then again had a relatively quiet time until the first decade of this century, when the production of electrical power from organic wastes, especially wastewaters, was identified as a unique opportunity for BESs. Production of electrical power from wastes is achieved by oxidation of organics, in combination with reduction of a high-potential, abundant chemical at the cathode ${ }^{[9]}$. Frequent cathode reactions applied were oxygen, ferricyanide and nitrate reduction ${ }^{[10-12]}$. As such, these systems functioned as microbially assisted fuel cells, and hence the name Microbial Fuel Cells (MFC) was born ${ }^{[13]}$.

Although holding great promises, the development of MFCs into successfully implemented technology was hindered by a barrage of practical difficulties, most notably (i) poor cathode performance under the operational restrictions set by microbial anodes ${ }^{[14]}$, (ii) inhibition of microbial activity by the formation of $\mathrm{pH}$ gradients inherent to proton-coupled redox reactions taking place in poorly buffered, low ionic strength electrolytes ${ }^{[15]}$ and (iii) side processes, mainly methanogenesis, desulfurization and oxygen reduction, scavenging substrate intended for production of electrical current ${ }^{[16]}$. Confronted with these challenges, the scientific community focusing on BES research has since then responded with an explosion in published research on these topics.

In addition to the continuing work on MFC improvement, research broadened as experiments were carried out in which a small external voltage was supplied to MFCs, allowing the production of hydrogen at their cathode. Although these systems require a net consumption of electrical power for producing hydrogen (thus not strictly adhering to the definition of a fuel cell), they do so to a lesser extent than conventional electrolysis and achieve this by using the assisting energy released by oxidation of organics at the anode - a concept termed microbial electrolysis ${ }^{[1,17]}$. Operating Microbial Electrolysis cells (MECs), the need for oxygen reduction or other high potential electron acceptors is avoided altogether. Therefore MEC has the advantage of avoiding the suboptimal performance sticking to high potential cathode reactions and also omits the requirements regarding expensive catalysts, especially when biocatalysis of the cathode reaction is applied ${ }^{[15]}$. Due to these 
advantages, interest in MEC research soon caught up with and possibly outpaced the efforts made on optimisation of MFCs. With the cathode not limiting overall reactor performance in MEC as much as it did for MFCs, this put back the research focus largely on anode performance, giving rise to further deepening of knowledge of and explorations on anode materials ${ }^{[18]}$, anode bacterial community composition dynamics $^{[19]}$ and optimization of operational conditions and cell design ${ }^{[16]}$.

After the pioneering work on MECs by Rozendal and Logan ${ }^{[15,17]}$ research also continued on the front of low potential biocathodes, and not long after production of hydrogen was shown to be catalysed by polarity-inversed anodic biofilms, the biocathodic production of reduced carbon compounds like methane and formic acid was reported ${ }^{[20,21]}$. This quickly developed to give rise to a completely reverse application of BESs, in which electrical power is converted into organic commodities using only inorganic compounds as substrate, a concept which then was termed Microbial ElectroSynthesis (MES). With the concept being named first in the context of biocathodic production of acetic acid by reduction of $\mathrm{CO}_{2}$, it is now more broadly used to describe biocathodic microbiomes producing a plethora of carbohydrates ${ }^{[22]}$.

With both production of electricity from organics by MFCs and the reverse conversion of electrical power to organics by MES proven to be possible, this paved the way for integration of the two, which would then act as an EST. This thesis describes the works on a first proof of concept of such a BES-based EST, and further experimental efforts taken for its optimization. As the work presented further will show, integration of MFC and MES in a single setup requires meticulous engineering to host the separate biological processes of both individual systems in a stable manner. Moreover, the main biological processes underlying both charging and discharging are studied in more detail, this in order to acquire useful information on their future optimization. Some of the main pitfalls as they apply to individual MFCs and MESs, apply also to MRBs, and include most importantly (i) sensitivity to changing electrolyte composition and (ii) side reactions as they result from alternative metabolic pathways and bacterial growth. Overcoming these challenges carries importance both for further development of the MRB, as for related BES technologies, and suggestions to this end are made in this thesis on the basis of presented results. At last, exploration of suitable counter electrodes is done to further improve the performance and sustainability of the MRB.

\subsection{WHAT MAKES A GOOD BATTERY?}

A good energy storage technology is one that fits its purpose. With applications in batteries ranging from wrist-watches and pacemakers to back-up UPSs powering complete hospitals, it is clear that there is no "one-size-fits-all" solution where it regards "the energy storage solution of the future". That said, as a generalized 
answer to the question what defines a good storage technology, could serve: one that fixes the mismatch between the demand and supply for electrical power most adequately for the lowest possible (long term) costs. Applying that view to grid energy storage, the mismatch itself becomes the point of attention. What would such a mismatch look like, once we start to (either voluntarily of forcibly) become more reliant on wind, hydro and solar energy?

\subsubsection{What will the mismatch look like?}

An useful exercise to understand the mismatch in terms of the directions it could take, and its consequences for suitable energy storage solutions, is to sketch the mismatch-landscape under hypothetical conditions. To visualize a mismatch in energy supply and demand, it is important to recognize the different time-scales in which mismatch is manifested. To illustrate this, imagine a single household in a temperate region with solar panels on it, for which the installed capacity of solar power is dimensioned to meet overall annual demands. Figure 1 depicts solar panel power yield and domestic power demand for a day both in summer and winter under these hypothetical conditions.

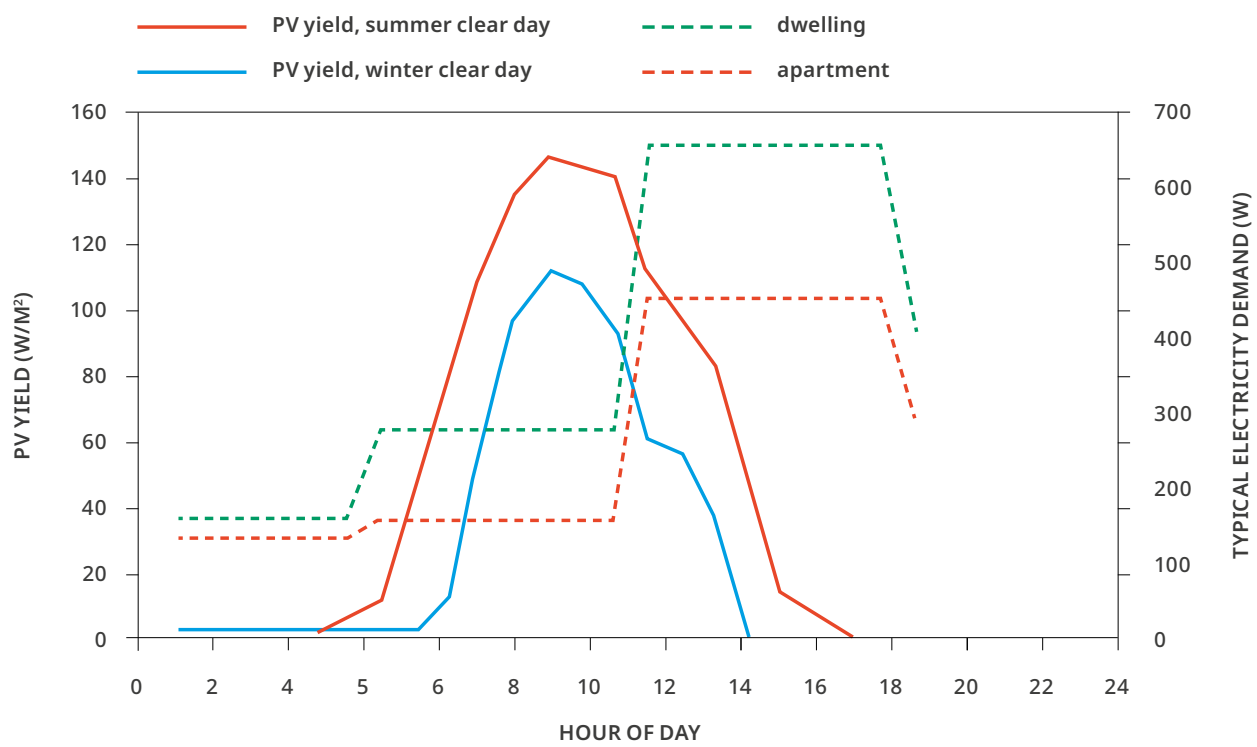

FIGURE 1 | Typical single-house power demand and supply profiles, assuming solar energy as the only electrical power source. Both summer and winter scenario is depicted. 


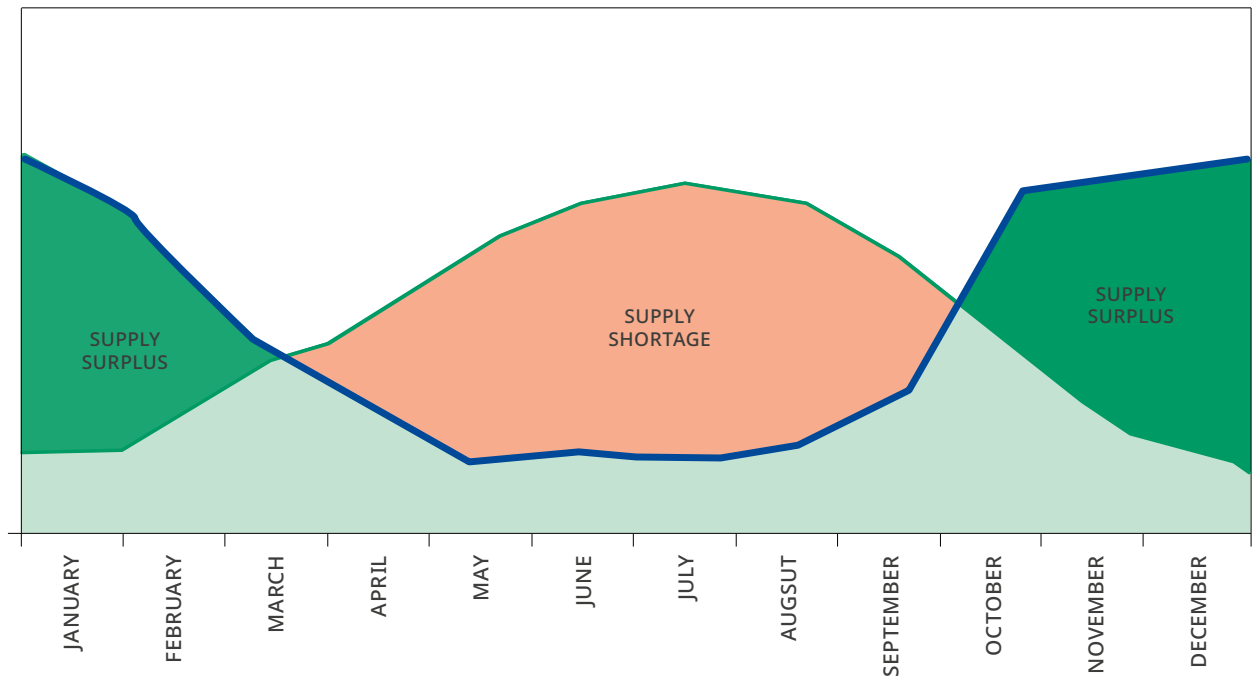

FIGURE 2 | Typical profiles of solar power supplied and heat demand for a single household on an annual basis.

Obviously, the largest part of mismatch is caused by diurnal changes in sunlight. When one would look on a more lengthier scale, e.g. days to months, a different picture results, depicted in Figure 2.

Likewise one can imagine that fluctuations in supply and demand, and thus mismatches, will exist on any time-scale, ranging from femtoseconds to years. The (relative) size of these fluctuations may not be relevant on every timescale: usually, on a femtosecond scale, larger systems have more than enough inertia to buffer, and apart from climate change, climatology has strong recurring annual patterns making analysis for more than 2-5 years less pressing. However, for the timescales that do bear relevance, in selecting the right storage technology the size and periodicity of these mismatches is of utmost importance. Just as the images above were constructed to visualize demand and supply characteristics for an extremely simple system consisting of just one supplier and one consumer, similar data could be compiled on any other spatial scale, and including multiple power sources. To illustrate this a bit further, Figure 3 shows a hypothetical small-scale wind and solar hybrid system, with wind (Pwind) and solar (Ppv) power yields plotted along with power demand for a $24 \mathrm{~h}$ interval.

Compared to the previous examples in which only solar power was included, the above graph shows less pronounced diurnal shortage and surplus. In this specific case, wind power to some extent operates in anti-phase compared to solar power(with most wind occurring at night time or during cloudy days). Further scaling up such observations in time, Figure 4 shows the power mismatch as it 


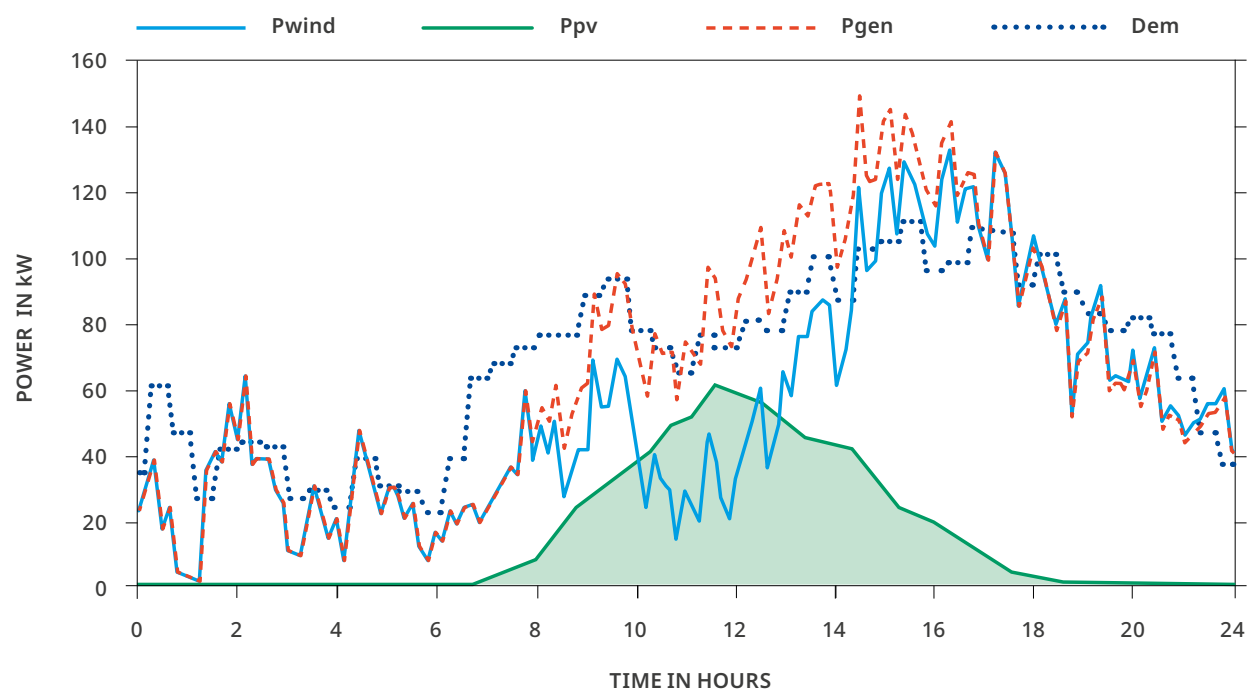

FIGURE 3 | Illustration of power supply and demands in a small scale wind-solar hybrid community. Power supplied by wind (Pwind), solar (Ppv) and combined power production (Pgen) is compared to power demand. Figure adopted from Fathima and Palanisami ${ }^{[32]}$.

results from the difference between total supply (Pgen in above graph) and demand (Dem) for a 10 day period:

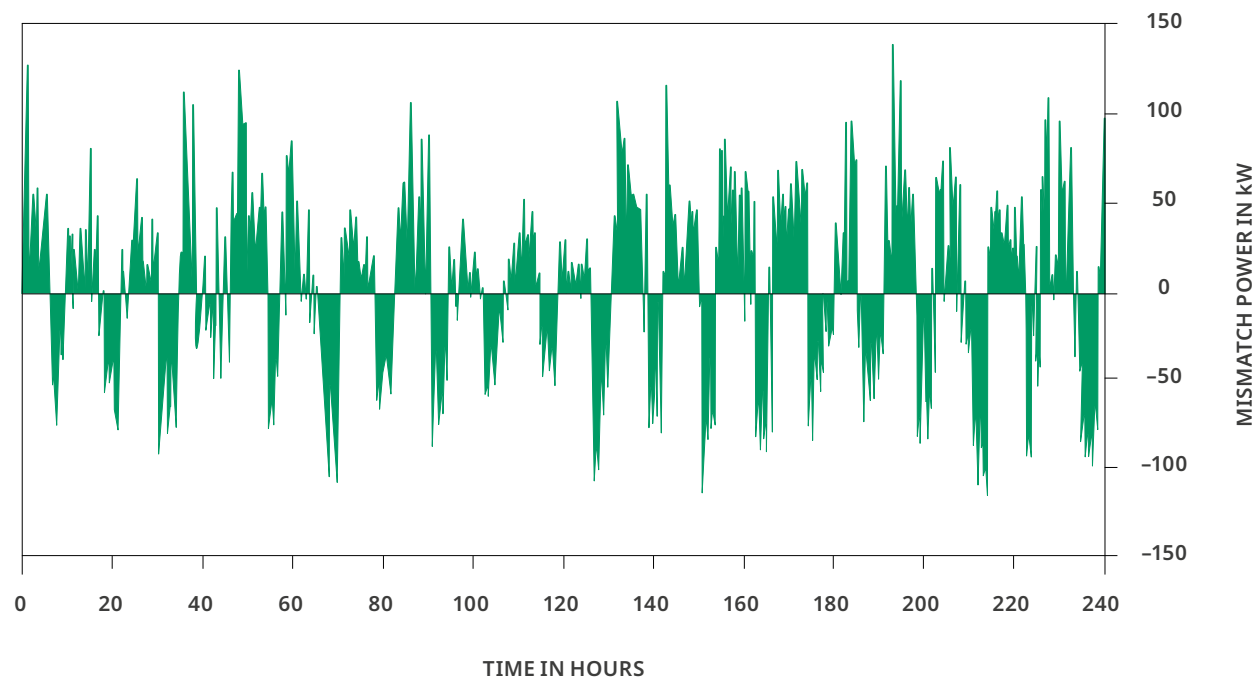

FIGURE 4 | Illustration of energy mismatch plausible to occurr in a small scale hybrid-solar renewable energy system. Figure adopted from Fathima and Palanisami ${ }^{[32]}$. 
It becomes visible that the larger the time scale, the higher the temporal resolution, and the more complex the system of energy suppliers and consumers, the more complex and rich the signal of energy mismatch as a function of time. For the above example for instance, a clear diurnal pattern remains visible (there are power surpluses and power shortages twice a day in an overall alternating pattern). However on top of this "regularity" you find spiky highs and lows, which do not seem so regularly structured.

By conducting time-frequency analysis on such mismatch signals, patterns may be identified and characterized. Although very sophisticated methods are developed to this end, details of which will not be treated here, it is easy to see that the outcome of such analysis will be very specific to the system analyzed. For example: wind power has a strong need for signal smoothing of moderately large drops and peaks in power production in the short frequency domain. As a result of these short frequency and moderate to high power density intermittency in wind generation, this would require small capacity energy storage located close to the turbines, but with a fast response. Typical backup technologies currently in use in combination with wind parks are therefore flywheels and supercapacitors for efficient and fast-ramping short frequency dampening. Additionally, large variation in power production by wind occurs over the course of multiple days, and smoothing of these low frequencies is conventionally achieved by compressed air energy storage (CAES), pumped hydro, or hydrogen storage. For these technologies, energy density weighs in much more than any other aspect, with less emphasis on fast response times. With PV, the most notable variation concerns the diurnal shifts, requiring 1 to 2 day acting, moderate to high power backup storage. The stochastic second-to-hour fluctuations in PV power caused by cloud formation are certainly a factor, but in amplitude less problematic as the gusts and lulls for wind. Especially in smaller scale situations (e.g. off-grid solar-only rural communities) in which PV installations are applied in practice, current available technologies often used for PV therefore are chemical battery technologies, like lithium-ion or lead-acid, to smoothen out both short and mid-term frequencies. In temperate regions, seasonal variation in both demand and supply comes in addition to aforementioned requirements, and hydrogen-based "electrofuels" have been mentioned to cater this future market ${ }^{[23]}$.

\subsubsection{The primary criteria for mitigation of mismatch: capacity and power}

The previous paragraph illustrated how mismatch may be very specific to each system element, with notable differences explicitly mentioned between wind and solar energy. To summarize the matter, Figure 5 provides a rough classification of system challenges as one probably could identify these on an overall mismatch 


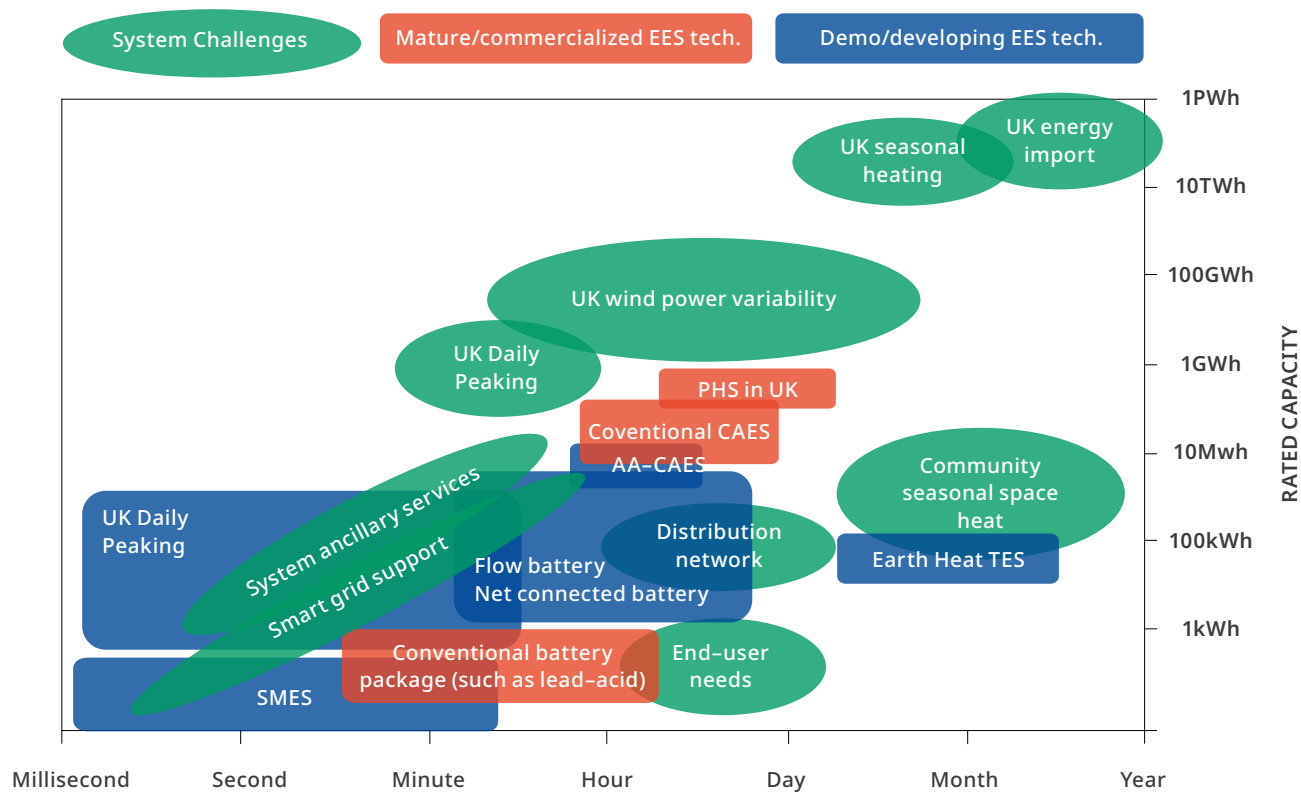

DISCHARGE TIME DURATION AT RATED POWER

FIGURE 5 | System challenges and applicable storage technologies specificied by total required capacity and power, based on a UK model, adopted from Luo et al ${ }^{[24]}$.

analysis for a large size modern-world energy grid with high levels of renewable energy penetration. In addition to the system challenges, ESTs are listed that may be able to address them. In this example, a scenario was worked out for the whole of UK, with the figure adopted from Luo et al ${ }^{[24]}$.

In Figure 5, depicted challenges are plotted as a function of energy capacity and discharge time duration at rated power. It is these two variables that form the primary technical aspects by which ESTs are judged. Challenges (or smoothing requirements) may be identified by conducting frequency analysis of mismatch curves (i.e. by studying their respective power spectral density plot). Doing so, one may obtain average mismatch power as it results from dynamics within certain frequency range(s). By dividing average mismatch power by frequency, one would obtain an estimate for the required capacity. With the inherent condition that the storage technology applied must be able to provide or take in the calculated capacity within the time duration defined by its frequency range, compatible storage technologies and demand modulating technologies can be listed likewise. The system challenges, orange boxes in Figure 5 above, are named after the physical phenomenon that creates most of the fluctuation or mismatch within the integrated frequency range. It has to be noted here that for several frequency 
ranges the required capacity has been diversified by listing multiple challenges (for instance, smart grid support and system ancillary services require technically identical countermeasures).

What is maybe most striking about Figure 5 is that at the moment no feasible technology (seems to) exist that would be able to store up quantities as high as they would be needed in the status-quo for seasonal heating and energy import. This is only partially the case, as theoretically various technologies could be applied at large scales (for instance hydrogen storage) for this purpose. The fact these solutions are not depicted mainly has to do with the fact that currently available technologies for bulk energy storage $>100 \mathrm{GWh}$ at feasible physical scale (which are almost exclusively hydrogen based) are characterized by low cycle efficiencies, typically lower than $40 \%$. As such, they would require massive upscaling on the supply side before becoming successfully implemented. Thus, although theoretically storage facilities with capacities high enough can be built, the authors of above figure did not deem it feasible enough to include them. As a mitigating factor, not necessarily the current demand for house/building heating would have to be met completely using electrical power: by using heat pumps in combination with thermal energy storage (e.g. ATES), waste heat (from industrial processes) and improved insulation, energy required in the form of electrical power for heating can be drastically reduced. However, this would also require infrastructural reforms ${ }^{[23]}$.

\subsubsection{A more in-depth analysis of energy storage performance indicators}

In the above example, a theoretically possible EST (hydrogen storage for seasonal variation) was not listed due to secondary considerations. This already points out there is more to a storage technology than just its capacity and discharge time of duration. In practice, an energy storage facility will take up space and it will require materials and energy both for its production, operation and omission. From these aspects, and how they relate to storage capacity and power delivered, follows a list of technical performance indicators, which in the end will all be taken into account when it comes down to choosing the right energy storage solution for the purpose. A comprehensive overview of these criteria could be (i) power and energy density, (ii) cycle (or round trip) efficiency and self-discharge and (iii) safety, stability and (iv) sustainability (both technically as ecologically). A short discussion on these indicators will follow below, while for a more detailed analysis on how current and future storage technologies compare to each other in relation to these aspect the reader is referred to some excellent reviews ${ }^{[25,26]}$. 


\subsubsection{Power and energy density: the Ragone plot}

Supposedly, power and energy density are the two most important technical specifications of an EST. Although in concept fairly straightforward, they are reported in varying dimensions. Power density, also named specific power, is the amount of power delivered per amount (volume or weight) of the storage technology considered. Distinction can be made in (i) peak (or maximum) power and (ii) rated power. The interchangeable use of both might cause some confusion regarding the comparativeness of these terms, hence their definition: with peak power is meant the maximum power a system can deliver, albeit just momentarily, without the system being (irreversibly) damaged or performing outside of its specified range. With rated power, the focus is put more on endurance, with the system being able to deliver it continuously throughout charging/discharging cycles. Although it would be possible for every EST to report on both interpretations of power density, which one is used depends mainly on the application. For flywheels and supercapacitors, aiming for short, intense and fast acting release and uptake of energy, conventionally peak power is used. Rated power in turn is often reported for devices associated to longer term storage technologies like fuel cells, combustion engines and batteries.

Less ambiguously, energy density, or specific energy, defines the total amount of energy available from full charge to full discharge, expressed per per amount of EST considered. Both power and energy density can be reported specific to either EST weight or volume, and which one is of interest depends mainly on the application, with weight playing lesser a role in stationary applications, vice versa for mobile applications.

What has by now become a classical way of comparing these properties amongst different ESTs, is the Ragone plot, plotting power density as a function of energy density. In its early days predominantly applied only for comparing battery technologies, this visualisation method is more and more applied to energy storage in general. Used and published first by David V. Ragone, metallurgist at M.I.T. in $1968^{[27]}$, it was initially used to compare battery technologies as they were applied to electrically powered vehicles. Although highly practical for that purpose, the Ragone plot actually buried within it a technical inconsistency: power and energy density are presented as independent variables, while in reality these are almost always correlated: for most thermodynamic processes the rate with which a process is taking place, is positively related to the specific rate with which entropy (heat) is created ${ }^{[28]}$. So unless when heat is the desired product (which it is not when strictly conversion back to electrical power is considered) lower energy density will result as an EST is operated at higher power density. Therefore, when plotting Ragone charts, one cannot but adopt practical values for power and energy density as they are used in existing systems. It is also for this reason Ragone plots depict specific ESTs in ranges, and not in a dot-like manner. The same holds for Figure 5, in which energy capacity was plotted versus time duration of discharge. 
In fact, these two figures are closely related, as discharge time duration at rated power may be obtained by dividing energy density by (rated) power density. As such, Figure 5 may be interpreted as a Ragone plot for which its horizontal axis has been divided by its vertical axis. Figure 6 provides an example of a more conventional Ragone chart, depicting some of the most known and important ESTs up to date. Note that in plotting graphs like this, incorporating also technologies in which energy density is decoupled from power density imposes a challenge on the draftsman: as a result of this, wide stretched ranges are depicted for chemical energy storage technologies (e.g. $\mathrm{H}_{2}$-driven fuel cells), with the ends only appearing due to pragmatic considerations, not theoretical ones.

Typically for a Ragone plot, different physical phenomena on which ESTs are based show up at distinctly different sites in the plot. The kinetic and electrostatic based technologies are concentrated on the left top to middle, thus featuring high power at low specific energy. The right bottom to middle is populated by "fuel based" chemical storage technologies, either driven by combustion or fuel cells.

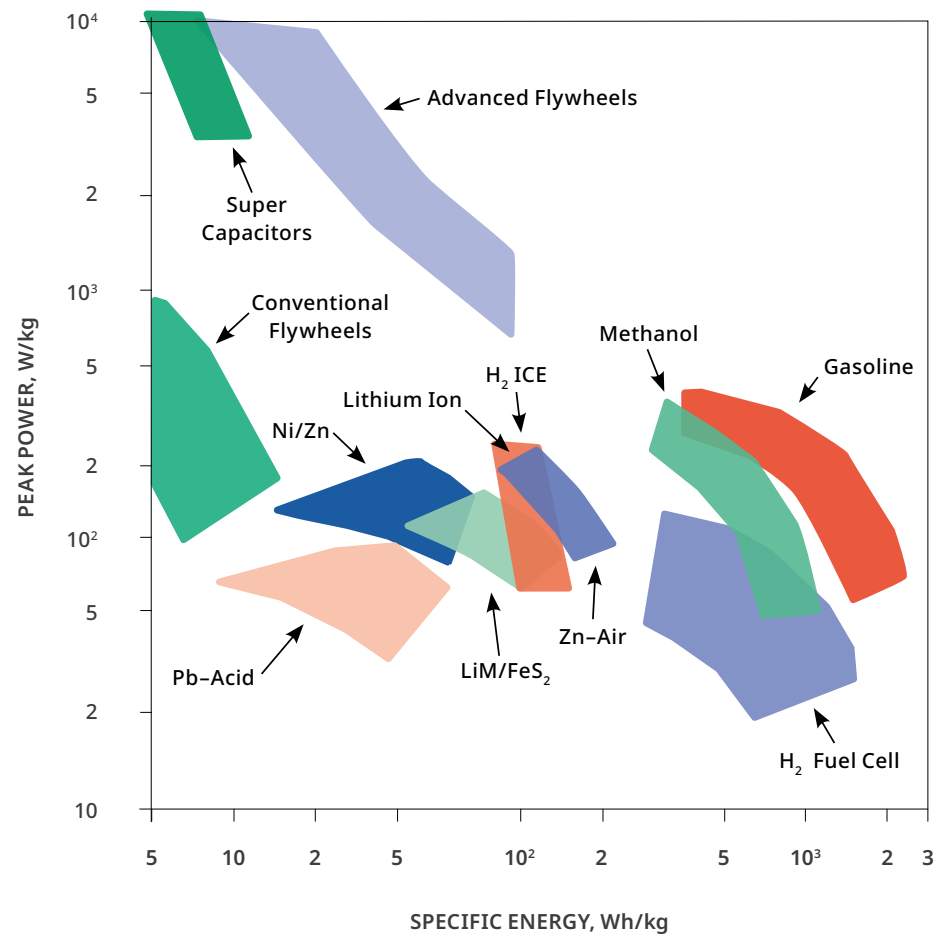

FIGURE 6 | The Ragone plot, used to compare energy and power densities of different Energy Storage Technology, historically especially in transportation systems, but applied nowadays more broadly. Figure adopted from Ghoniem ${ }^{[29]}$. 
Most battery technologies, both intercalation and redox based, are found in the center of the plot. As a result, though given its boundaries, the Ragone plot provides a helpful tool in quick selection of ESTs.

\subsubsection{Cycle efficiency and self-discharge}

Cycle efficiency of an EST (also named round trip efficiency or energy efficiency) is defined as the ratio between the amount of energy delivered upon discharging, to the energy absorbed upon charging. Like with energy density, the obtained energy efficiency is not a fixed entity for each specific technology, but depends on the conditions applied. In most cases cycle efficiency is mainly a function of the power density at which an EST is applied. In general, cycle efficiency goes down when an EST is operated closer to its maximum power density, as more heat is generated while charging and discharging it. As was mentioned for the correlation between energy and power density, this can be led back to the occurrence of entropy producing processes, and may be best compared to driving a car: higher mileages are obtained at lower and more steady speed.

Self-discharge determines the amount of stored energy lost over time as the EST is not put to work. The processes leading to self-discharge vary with each technology, and so does the exact method by which self-discharge is reported. However, with most ESTs, the mathematical description of the physical phenomenon leading to self-discharge allows the retained energy to be expressed in terms of a gradient. Very characteristic to a gradient, decay will occur in an exponential manner. Therefore, self-discharge rates can be reported in most cases per unit of time.

Obviously, there is a close connection between cycle efficiency and selfdischarge: an EST with high self-discharge can only be applied efficiently in the short frequency range. When used outside of its intended periodicity (for instance when flywheels would be used for storage over days) this would be disastrous to its cycle efficiency. Cycle efficiency is therefore conventionally reported at the rated power density and frequency of use.

Cycle efficiency especially has a very strong and direct effect on the cost effectiveness of an EST, as it is competing to the alternatives of grid interconnection or increasing production capacity. It is most likely for this reason that in current standing practice, technologies with cycle efficiencies below 50-60 \% are seldomly implemented.

To get an intuitive feel about how available and developing ESTs compare with regards to cycle efficiency and self-discharge, alike the Ragone plot, Figure 7 shows reported cycle efficiencies as function of self-discharge. Only power-topower technologies are mentioned (thermal energy storage omitted). 


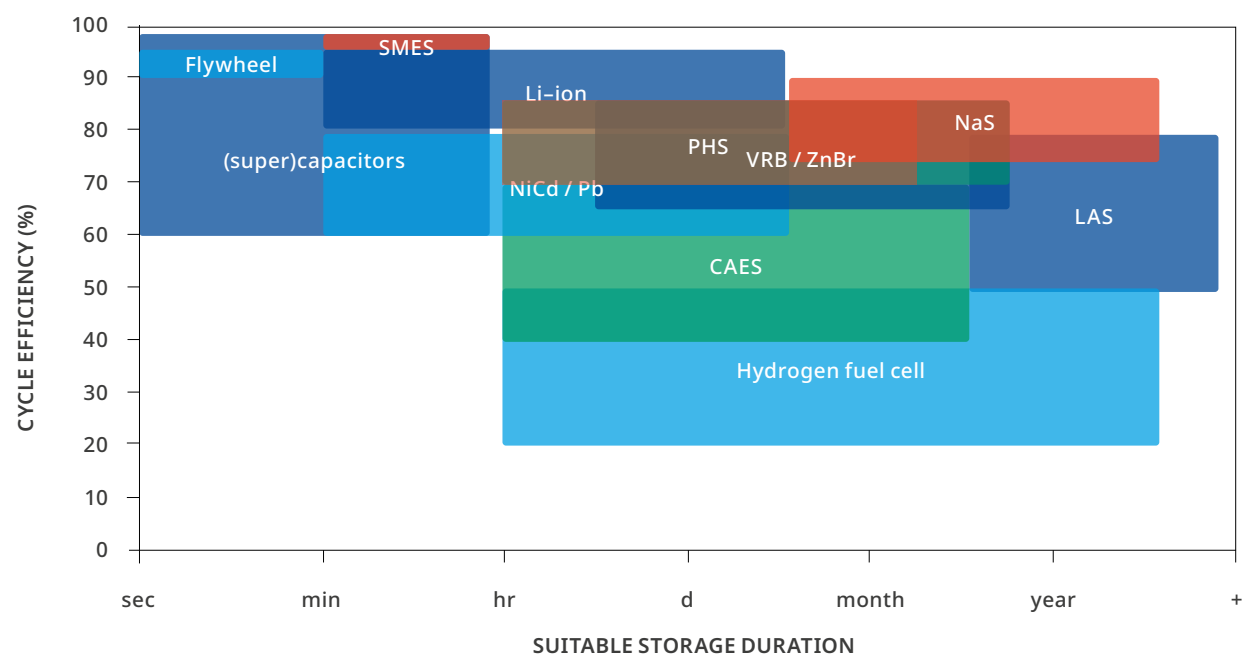

FIGURE 7 | General ranges of cycle efficiency versus suitable storage duration, where suitable storage duration is dictated primarily by self-discharge rates, for various ESTs. Tabulated values were obtained from reviews in literature ${ }^{[24]}$. Included in figure are: (i) kinetic (flywheels), (ii) gravitational/mechanical (pumped hydro; PHS, compressed air; CAES, liquid air; LAS), (iii) electrostatic (capacitors), (iv) electromagnetic (Superconducting magnetic; SMES, (v) redox-chemistry (flow cells; VRB/ZnBr, batteries; Li-ion/ $\mathrm{NiCd} / \mathrm{Pb} / \mathrm{NaS}$, fuel cells; Hydrogen Fuel Cells)

\subsubsection{Safety and stability}

The materials from which a technology is composed, and the conditions under which these are used, are major determining factors regarding the safety, stability and sustainability of a technology. Often seen as of secondary importance, these aspects are essential for allowing a proper long-term cost/benefit analysis of ESTs. A brief overview of the most prominent factors to take into account is provided.

\subsection{Technical sustainability: technology lifetime and maintenance costs}

Technology lifetime and maintenance costs cover the most straightforward sustainability aspects of a technology. Once it is manufactured, how long will it last? Depending on the characteristics of the storage technology, focus may be put on either wear and tear (i.e. in case of the moving parts found in kinetic systems like flywheels, or pumped hydro) or the occurrence of irreversible chemical processes leading to capacity loss or inactivation (batteries, flow cells). Additionally, each EST will require specific electronics to be connected and operated. These "hookup” electronics may actually form a substantial part of the implementation (e.g. system monitoring, AC/DC conversion etc.) and their lifetime specifics thus also need to be considered. Although for most technologies, prolongation of their 
lifetime is the focal point for $\mathrm{R} \& \mathrm{D}$, there are considerable up-front differences between technologies on these grounds. Technology lifetime is often reported both as "on the shelf life time" (thus time-dependent) and cycle lifetime (the amount of charge/discharge cycles minimally guaranteed before a certain loss of function, thus use-dependent).

\subsection{Safety}

Intuitively one can feel that whenever high amounts of energy are concentrated in a small space, it becomes increasingly important to properly control the rate of release. Would control be lost, excessive heat production, and thus fires and explosions, are the most logical direct result. Some examples for conventional systems: flywheels shatter, gas canisters may explode, batteries may have a thermal runaway causing a meltdown or even explosions, and those are the short term disadvantages. Especially true for electrochemical systems, these have a tendency of requiring substantial amounts of reactive metallic compounds for their production. In addition to this, most conventional electrolytes are nonaqueous, instead based on mineral oils or polymers thereof (non-biodegradable, explosive and often carcinogenic). As such, both during production, operation and recycling/deconstruction of these devices, large health and environmental risks have to be contained properly. Although safety in the first place is something that can be addressed by taking proper measures, the necessity to take such measures varies per EST and co-defines it economic position.

\subsubsection{Ecological footprint: energy, ecology and land-use}

With human caused change in land-use being the primary reason for the recent and ongoing biodiversity mass extinction, ESTs requiring more land-use change for their production or operation are to be avoided. From this perspective, low specific energy storage technologies that interfere with land-use directly and on a large scale like pumped hydro (requiring dams and inundation) or cavern-based compressed air energy storage are severely restricted regarding future upscaling. However, also for ESTs with higher energy density, land-use change is often an issue to not be overlooked: in addition to the toxicity threats associated with the use of heavy metals and fossil sources as described above, the mining and extraction of these resources also impose a direct danger to natural ecosystems: regardless of how efficient one can mine, often the elements of interest occur in relatively low concentrations and require the handling and processing of considerable volumes and hence surface area (of either ores, seawater, air) to be obtained.

Striving for minimum ecological impact, it is thus generally a bad idea to use scarce resources for the EST of the future. For a more elaborated view on material selection criteria in light of their sustainability the reader is referred to the recent work of Carmona et $\mathrm{al}^{[30]}$. 


\subsection{MRB FUNDAMENTALS}

Using BESs, organic redox couples often including complex and multi-electron transfer reactions, can be catalysed at conditions and efficiencies that weren't imagined before. Typically for most biological systems, BESs are operated under "mild" conditions, compared to abiotic electrochemistry: electrolytes are without exception aqueous, contain relatively low concentrations of solutes, make use of abundant chemicals and systems are operated at moderate temperatures (most typically 20 to 35 degrees Celcius, with exceptions in case extremophilic organisms are used). The microbes hosted in these systems, after being inoculated, grow, maintain, and potentially regrow themselves, thus effectively acting as autocatalysis.

Figure 8 presents a general schematic of a BES. As such it depicts a basic electrochemical system, with the anode and cathode being separated by a membrane. Conventionally, the anode oxidizes a reduced substrate (electron donor) into an oxidized product, delivering electrons to the anode. The cathode reduces an oxidized electron acceptor into a reduced product, an electron consuming process. The two half reactions are coupled by an electrical circuit allowing for an electron flow from anode to cathode. Unique for BESs is the presence of bacteria (here depicted as biofilm) catalysing these reactions on or at the anode and cathode, but it should be stressed that any electrochemical

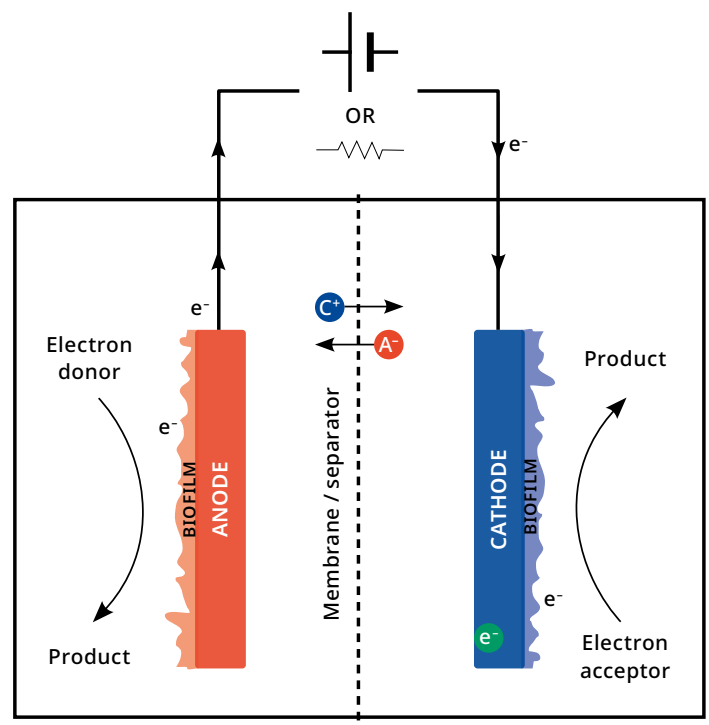

FIGURE 8 | General schematic for a bioelectrochemical system depicting a classical electrochemical configuration with electrode-metabolizing biofilms on anode and cathode. 
system in which at least one of the electrode reactions of interest is catalysed by living bacteria can be regarded a bioelectrochemical system. As was already pointed out in the previous paragraph, nowadays multiple branches exists within this discipline: BESs delivering a net output of electrical energy are called microbial fuel cells (MFCs), and practically always contain a biotic anode, combined with an either a biotic or abiotic cathode. For these systems, the external electrical circuit can be interpreted as a resistor (lower icon). BESs that require externally added electrical power for their functioning (thus with the electrical circuit connected to a power source, upper icon) are either called microbial electrolysis cells (MECs, in the specific case of a biotic anode combined with a biotic or abiotic cathode producing hydrogen) or microbial electrosynthesis (MES, in all other cases).

As a net electron flow is occurring from anode to cathode, charge neutrality in the system has to be maintained. This is done by an accompanying exchange of ions between the cathode and anode compartments. Depending on what kind of membrane is used, selectivity towards transport of certain ion species is achieved. Most prominent examples to be found in literature herein are: (i) anion selective exchange membranes, (ii) cation selective exchange membranes, (iii) bipolar membranes, (iv) mosaic membranes (both permeable to cations and anions, but less permeable to uncharged and/or larger compounds, (v) (J-)cloth (no selectivity, only stopping convection), (vi) no membrane at all[31]. Which type of separator is used depends on the objectives of the system (e.g. for fuel cells low resistance is prime, while for electrosynthesis purity of products and thus membrane selectivity is more important).

In the specific situation of a Microbial Rechargeable Battery (MRB) the system is composed as a combination of a MFC and MES: during charging of the MRB, the systems functions as a MES, converting electrical power delivered by an applied voltage into biochemical commodities. During discharge, the system releases electrical energy to an external electrical circuit by biological oxidation of organics in the anode, and functions as a MFC. For a detailed schematic of the workings of the MRB see Chapter 2.

\subsubsection{Main factors affecting MRB performance}

To study the performance of the MRB separately throughout the phases of charging and discharging, and compare its performance in these two distinct phases with what could be expected based on equilibrium thermodynamics, some standard efficiency calculations have been developed in the field. These can be described on the bases of certain losses. Losses in electrochemical systems in general can be divided in two distinct categories: (i) energetic losses by production of heat (entropy) and (ii) coulombic losses, which occur by means of redox processes not 


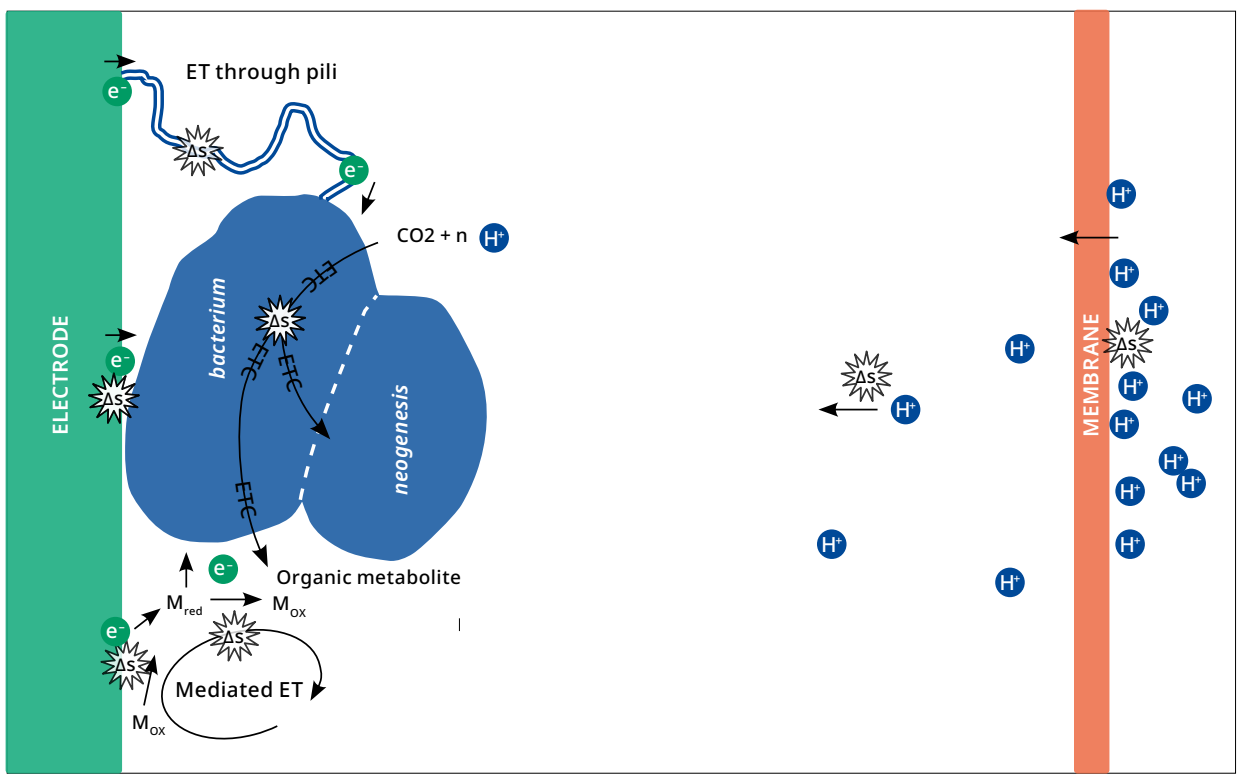

FIGURE 9 | Schematic overview of possible electron/charge trajectories in BES and associated losses and entropy generating processes (indicated by explosion symbols). Diffusion energy losses occur when free charges are moving through non-crystalline matrices (e.g. ions through electrolytes or membranes). Energy losses by means of electrical resistance (electron tunnelling) occurs whenever electrons are traveling through metallic matrices (e.g. within the electrode material, or supposedly through conductive pili). Energy dissipation to heat can also occur when electrons are transferred from nucleus to nucleus, or from nucleus to electrode (e.g. at the electrode in the case of mediated ET or intercellularly throughout the electron transfer chains (ETC), as well as in case of direct contact of the cell membrane with the electrode, in which case membrane bound redox active proteins may do the trick. In these latter cases, Marcus theory would be a fitting description. Coulombic losses occur when electrons are directed towards processes not of interest to the technologies primary goal (for instance in the case of biomass growth, neogenesis).

contributing to the final aim of the system. A schematic overview of these losses is provided in Figure 9, and methods for identification and measurement are discussed below.

\subsubsection{Energetic losses (kinetic or voltage losses)}

The main aspect of any electrochemical system is its voltage: the higher the voltage, the more energy delivered/invested per charge transferred. Although in BESs the obtained electrode potentials, and thus cell voltage, are a result of many factors including metabolic diversity and degree of electrode colonization, the theoretical values for anode and cathode potentials during open circuit 
conditions, thus based on the concept of chemical equilibrium, can be calculated by the Nernst formula:

$$
\mathrm{E}_{\text {electrode,eq }}=\mathrm{E}_{0}-\frac{\mathrm{F}}{2}
$$

With $\mathrm{E}_{0}$ being the standard reduction potential of the half-reaction of interest, $\mathrm{R}$ the gas constant, $\mathrm{T}$ the temperature in Kelvin, $\mathrm{z}$ the number of electrons involved in the half reaction, $\mathrm{F}$ the faraday constant, and $\mathrm{Q}$ the reaction quotient including the activities of the chemical compounds involved in the half reaction.

After individual values for anode and cathode are calculated by above formula, the equilibrium cell voltage is further expressed as:

$$
\mathrm{E}_{\text {cell }, \text { eq }}=\mathrm{E}_{\mathrm{cat}, \mathrm{eq}}-\mathrm{E}_{\mathrm{an}, \mathrm{eq}}
$$

In practice however, this equilibrium cell voltage will never be measured (not even at open circuit conditions, thus with practically no current drawn) in biotic systems as every current, even a small one required for obtaining measurements on an electrochemical system, will require an off-equilibrium situation to be drawn. A deviation in electrode potential as expressed to the (formal) equilibrium potential, which by means of the second law of thermodynamics is required for a current to start flowing, is conventionally named overpotential. In the case of BESs, the biocatalyzed reactions involved are often complex and consist out of a cascade of conformational steps, typical to enzyme-driven electron transfer chains (ETCs). Apart from just "turning over molecules and electrons" these ETCs are responsible for the production of ATP by phosphorylation. As this requires exergy, a current "drawn through a biological system" by definition will have (considerable) overpotentials, even when measured at near-equilibrium conditions (i.e. at negligible current density). Another way to look at it is as follows: it is not the formal potential of the initial electron donor/acceptor that defines measured electrode equilibrium potential in a BES, but the final electron donor/acceptor as it actually interacts with the electrode. The chemical structure and properties of the final electron donor/acceptor however is often unknown, subject to change as a result of microbial metabolic dynamics, and therefore subject to study. Apart from energetic losses as they occur as a result of biochemical processes taking placing within the living cell, BESs are subject to physicochemical-induced losses just like conventional electrochemical systems are. The combined effect of these losses, thus all inducing certain overpotentials, is that the cell voltage in practice turns out smaller (in case of an MFC) or larger (in case of an MES) than what would be expected based on their thermodynamic equilibria. By measuring this discrepancy in real systems, the total energetic losses/overpotential can thus be described as: 


$$
\mathrm{E}_{\text {cell ,eq }}-\mathrm{E}_{\text {cell ,measured }}=\eta
$$

Once the total overpotential ( $\eta$ ), resulting from all of bespoken losses, of the entire electrochemical system (thus both for anode and cathode, and at a given current density) is known, the voltage efficiency (VE) is defined, depending on whether a charging or discharging situation is considered, as:

$$
\begin{gathered}
\mathrm{VE}_{\text {charging }}(\mathrm{MES})=\frac{\mathrm{E}_{\text {cell }, \mathrm{eq}}}{\mathrm{E}_{\text {cell ,eq }}+\eta} \\
\mathrm{VE}_{\text {discharging }}(\mathrm{MFC})=\frac{\mathrm{E}_{\text {cell ,eq }}-\eta}{\mathrm{E}_{\text {cell ,eq }}}
\end{gathered}
$$

\subsubsection{Coulombic losses (electrons lost to the process of interest)}

The second most important parameter of electrochemical systems regards their Coulombic efficiency (CE). Where voltage efficiency dealt with the amount of energy lost on a per electron basis due to heat dissipation, Coulombic losses represent the diversion of energy towards processes leading to products that are considered useless or just not contributing to the technologies' purpose.

Thereby, the CE expresses the amount of electrons ultimately supplied to the system in relation to the amount of electrons recovered in the electron acceptor of interest:

$$
\mathrm{CE}=\frac{\text { total charge recovered in acceptor of interest }}{\text { total charge supplied by donor }}
$$

Depending on whether CE is expressed for a MFC or MES, its formal definition changes slightly. For an MFC, where the objective is to generate electric current for power production, $\mathrm{CE}$ is defined as:

$$
\mathrm{CE}_{\mathrm{MFC}}=\frac{\text { total charge recovered as electric current }}{\text { total charge available by a measured decrease in substrate }}
$$

For MES, where the aim is to produce reduced compounds at the cathode, the CE is expressed as:

$$
\mathrm{CE}_{\mathrm{MES}}=\frac{\text { total charge required for synthesizing a measured amount of product }}{\text { total charge delivered to the cathode by a measured current }}
$$


Ideally, CE would be 1, in which case all electrons theoretically available to the system end up in the compound/process of interest. However, due to the inevitable consumption of electrons by microorganisms in BESs as they are used for biomass production, and by parasitic processes often occurring in anaerobic systems, using alternative electron acceptors, most notably methane production, CEs in practice are less than 1.

\subsubsection{Energy Efficiency}

When $C E$ and $V E$ are known, they can be multiplied to express the energy efficiency (EE) of a BES:

$$
\mathrm{EE}=\mathrm{CE} \cdot \mathrm{VE}
$$

For the MRB, this allows separate calculation of the EE both during the charging (MES) and discharging (MFC) phase:

$$
\begin{aligned}
& \mathrm{EE}_{\mathrm{MES}}=\mathrm{CE}_{\mathrm{MES}} \cdot \mathrm{VE}_{\mathrm{MES}} \\
& \mathrm{EE}_{\mathrm{MFC}}=\mathrm{CE}_{\mathrm{MFC}} \cdot \mathrm{VE}_{\mathrm{MFC}}
\end{aligned}
$$

In case the energy efficiency of the MRB is considered as a whole, thus defined as the Cycle Efficiency (or round-trip efficiency), it would be calculated by:

$$
\text { Cycle Efficiency }=\mathrm{EE}_{\mathrm{MFC}} \cdot \mathrm{EE}_{\mathrm{MES}}
$$

Although the EE may form the most fundamental performance indicator, expressing the actual amount of Joules ending up in the desired process or compound as compared to the amount of Joules available to the system, given the often dynamic characteristics of CE and EE throughout system operation, it is calculated on short time intervals, and its quantity is thus most practically stated as a systems average throughout operational periods.

\subsection{RESEARCH OBJECTIVE AND THESIS OUTLINE}

The objective of this thesis is to develop a sustainable, efficient and powerful energy storage technology by combining an acetate-oxidizing MFC with an $\mathrm{CO}_{2}$-reducing MES, giving rise to a Microbial Rechargeable Battery. In order to do so, a choice had 
to be made regarding the (initial) counter electrode. In Chapter 2, a proof of concept of such a MRB using ferricyanide at the counter electrode is provided.

The main challenge in providing the proof of concept was in designing and operating the system in a way that it could host both anodic and cathodicactive biota, operating each intermittently. As an outcome, in Chapter 2 the electrochemical dynamics of the developed system are reported, and based on these, main limiting factors are identified, providing possible future strategies for improvement.

In order to further develop the MRB towards a mature energy storage technology, an important challenge is to find a balance between energy invested into keeping microorganisms growing (we need them!), and what we get back for it. One of the main energy losses in the MRB as it occurs over time are Coulombic losses caused by production of methane by unwanted methanogenic growth. Chapter 3 and Chapter 4 describe in more detail the effect electrode potential and substrate concentration may have on process selectivity, through microbial competition. The outcomes of these studies provide directions towards more optimized startup, charging and discharging of a future MRB, and how this may contribute to increased system stability and selectivity.

Although using microbes as catalysts is much praised in terms of system resilience, self-repair, simplicity and low-costs; growth and activity of microbes requires energy and mass (through carbon fixation). These energy and mass losses translate to both voltage and Coulombic efficiency losses in the MRB. At least to some extent inevitable, to which degree these losses occur is dependent on the current density and/or electrode potential at which the system is operated. The latter is a result of the metabolic adaptability of bacteria. To be able to study the adaptability of microorganisms' growth towards changing operational parameters in more detail, a methodology was developed to track biofilm growth in BESs real-time and non-invasively, using Optical Coherence Tomography (OCT). The results of these developments and its implications are treated in Chapter 5.

The major energy losses described for the MRB in chapter 2 could be ascribed to $\mathrm{CE} / \mathrm{VE}$ losses occurring in the biological electrodes. However, energy density of the initially developed system was limited mainly by the limited solubility of the ferricyanide couple with which the proof-of-concept was delivered. Moreover, practical difficulties and stability issues related to the use of ferricyanide made the search for more sustainable - and hopefully more powerful - counter electrode reactions worthwhile. A further exploration to this end, covering the integration of both a capacitive and oxygen/water electrode in the MRB platform is dealt with in Chapter 6. 
In addition to the inevitable energetic losses resulting from the growth and maintenance of microorganisms, when employing microbes for conducting electrochemical conversions this comes with restrictions set towards operational conditions and system design. Much praised as inherently safe and environmentally friendly catalysts, biological activity requires relatively narrow ranges in operational conditions that are often in conflict with high performance. To name a few of the most prominent restrictions: narrow temperature ranges, (near)neutral $\mathrm{pH}$, moderate concentrations of electrolytes (both substrates, products, and other ions). These restrictions may severely impact the attainable reaction rates and thus power density, as is the case with temperature and concentrations as it leaves less room for improvement in alleviating mass transfer related losses. Alternatively, higher charge densities and thus energy densities may not be obtained as this would require reactant concentrations regarded to be toxic to the present biota. In Chapter 7, an overview of these restrictions and limitations as they have occurred in the works described in earlier chapters will be reviewed, and an outlook is provided regarding possible future workarounds/optimizations. 


\subsection{REFERENCES}

(1) Denholm, P.; Hand, M. Grid Flexibility and Storage Required to Achieve Very High Penetration of Variable Renewable Electricity. Energy Policy 2011, 39 (3), 1817-1830.

(2) Lund, P. D.; Lindgren, J.; Mikkola, J.; Salpakari, J. Review of Energy System Flexibility Measures to Enable High Levels of Variable Renewable Electricity. Renew. Sustain. Energy Rev. 2015, 45, 785-807.

(3) Denholm, P.; Ela, E.; Kirby, B.; Milligan, M. The Role of Energy Storage with Renewable Electricity Generation. Natl. Renew. Energy Lab. 2010, 1-61.

(4) Larcher, D.; Tarascon, J. M. Towards Greener and More Sustainable Batteries for Electrical Energy Storage. Nat. Chem. 2015, 7 (1), 19-29.

(5) Wikipedia Contributors. Life-cycle assessment https://en.wikipedia.org/wiki/Life-cycle_assessment (accessed Jul 18, 2018).

(6) Rabaey, K.; Rozendal, R. A. Microbial Electrosynthesis - Revisiting the Electrical Route for Microbial Production. Nat. Rev. Microbiol. 2010, 8 (10), 706-716.

(7) Potter M.C. Electrical Effects Accompanying the Decomposition of Organic Compounds. Proc. R. Soc. London. Ser. B, Contain. Pap. a Biol. Character 1911, 84 (571), 260 LP-276.

(8) Davis, J. B. Generation of Electricity by Microbial Action. Adv. Appl. Microbiol. 1963, 5 (C), 51-64.

(9) Logan, B. E. Simultaneous Wastewater Treatment and Biological Electricity Generation. Water Sci. Technol. 2005, 52 (1-2), 31-37.

(10) Rabaey, K.; Read, S.; Clauwaert, P. Cathodic Oxygen Reduction Catalyzed by Bacteria in Microbial Fuel Cells. ISME ... 2008, 2 (5), 519-527.

(11) Bergel, A.; Féron, D.; Mollica, A. Catalysis of Oxygen Reduction in PEM Fuel Cell by Seawater Biofilm. Electrochem. commun. 2005.

(12) Clauwaert, P.; Rabaey, K.; Aelterman, P.; Schamphelaire, L. De; Pham, T. H.; Boeckx, P.; Boon, N.; Verstraete, W.; Clauwaert, P. Biological Denitrification in Microbial Fuel Cells. 2007, 41 (9), 3354-3360.

(13) Logan, B. Microbial Fuel Cells; Wiley, 2008

(14) Heijne, A. Improving the Cathode of a Microbial Fuel Cell for Efficient Electricity Production, Wageningen University, 2010.

(15) Rozendal, R. Hydrogen Production through Biocatalyzed Electrolysis, Wageningen University, 2007.

(16) Sleutels, T. H. J. A.; Darus, L.; Hamelers, H. V. M.; Buisman, C. J. N. Effect of Operational Parameters on Coulombic Efficiency in Bioelectrochemical Systems. Bioresour. Technol. 2011, 102 (24), 11172-11176.

(17) Logan, B. E.; Call, D.; Cheng, S.; Hamelers, H. V. M.; Sleutels, T. H. J. A.; Jeremiasse, A. W.; Rozendal, R. A. Microbial Electrolysis Cells for High Yield Hydrogen Gas Production from Organic Matter. Environ. Sci. Technol. 2008, 42 (23), 8630-8640.

(18) Sonawane, J. M.; Yadav, A.; Ghosh, P. C.; Adeloju, S. B. Recent Advances in the Development and Utilization of Modern Anode Materials for High Performance Microbial Fuel Cells. Biosens. Bioelectron. 2017, 90, 558-576.

(19) Yates, M. D.; Kiely, P. D.; Call, D. F.; Rismani-Yazdi, H.; Bibby, K.; Peccia, J.; Regan, J. M.; Logan, B. E. Convergent Development of Anodic Bacterial Communities in Microbial Fuel Cells. ISMEJ. 2012, 6 (11), 2002-2013.

(20) Lovley, D. R.; Nevin, K. P. A Shift in the Current: New Applications and Concepts for Microbe-Electrode Electron Exchange. Curr. Opin. Biotechnol. 2011, 22 (3), 441-448. 
(21) Nevin, K. P.; Woodard, T. L.; Franks, A. E. Microbial Electrosynthesis : Feeding Microbial Electrosynthesis : Feeding Microbes Electricity To Convert Carbon Dioxide and Water to Multicarbon Extracellular Organic. Am. Soc. Microbiol. 2010, 1 (2), 1-4.

(22) Schröder, U.; Harnisch, F.; Angenent, L. T. Microbial Electrochemistry and Technology: Terminology and Classification. Energy Environ. Sci. 2015, 8 (2), 513-519.

(23) Connolly, D.; Lund, H.; Mathiesen, B. V. Smart Energy Europe: The Technical and Economic Impact of One Potential 100\% Renewable Energy Scenario for the European Union. Renew. Sustain. Energy Rev. 2016, 60, 1634-1653.

(24) Luo, X.; Wang, J.; Dooner, M.; Clarke, J. Overview of Current Development in Electrical Energy Storage Technologies and the Application Potential in Power System Operation. Appl. Energy 2015, 137, 511-536.

(25) Luo, X.; Wang, J.; Dooner, M.; Clarke, J. Overview of Current Development in Electrical Energy Storage Technologies and the Application Potential in Power System Operation. Appl. Energy 2014, 137, 511-536.

(26) Zakeri, B.; Syri, S. Electrical Energy Storage Systems: A Comparative Life Cycle Cost Analysis. Renew. Sustain. Energy Rev. 2015, 42, 569-596.

(27) Ragone, D. V. Review of Battery Systems for Electrically Powered Vehicles. SAE International 1968.

(28) Martyushev, L. M.; Seleznev, V. D. Maximum Entropy Production Principle in Physics, Chemistry and Biology. Phys. Rep. 2006, 426 (1), 1-45.

(29) Ghoniem, A. F. Needs, Resources and Climate Change: Clean and Efficient Conversion Technologies. Prog. Energy Combust. Sci. 2011, 37 (1), 15-51.

(30) Carmona, L. G.; Whiting, K.; Carrasco, A.; Sousa, T.; Domingos, T. Material Services with Both Eyes Wide Open. Sustain. 2017, 9 (9).

(31) Logan, B. E.; Hamelers, B.; Rozendal, R.; Schröder, U.; Keller, J.; Freguia, S.; Aelterman, P.; Verstraete, W.; Rabaey, K. Microbial Fuel Cells: Methodology and Technology. Environ. Sci. Technol. 2006, 40 (17), 5181-5192.

(32) Fathima, A. H.; Palanisamy, K. Energy Storage Systems for Energy Management of Renewables in Distributed Generation Systems. Energy Management of Distributed Generation Systems. 2016 DOI: $10.5772 / 62766$. 


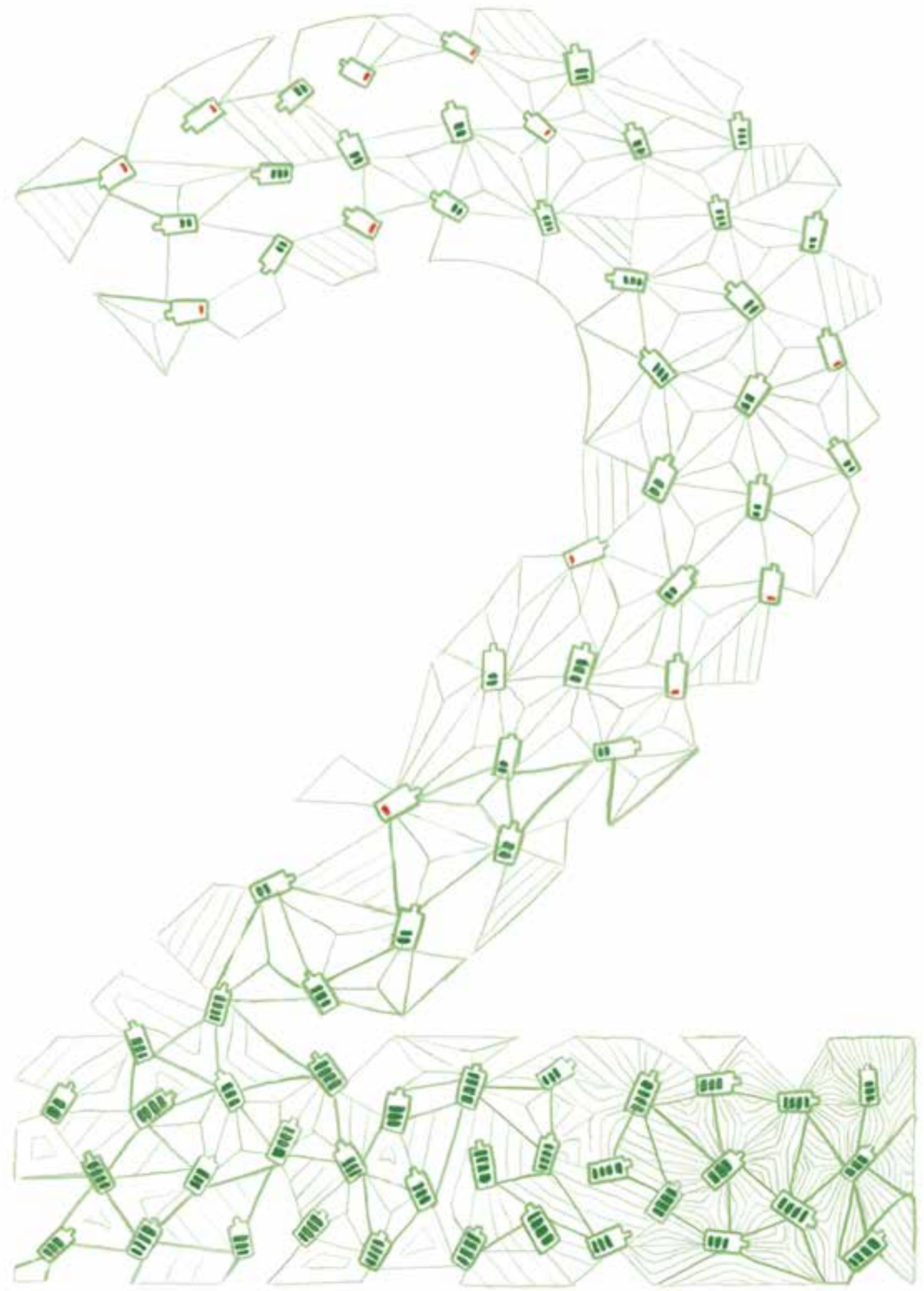




\section{THE MICROBIAL RECHARGEABLE BATTERY: ENERGY STORAGE AND RECOVERY THROUGH ACETATE}

Bioelectrochemical systems (BES) hold potential both for conversion of electricity into chemicals through microbial electrosynthesis (MES) and the provision of electrical power by oxidation of organics using microbial fuel cells (MFC). The work described in this chapter provides a proof-of-concept for a Microbial Rechargeable Battery (MRB) allowing storage of electricity by combining MES and a MFC in one system. Hexacyanoferrate (II/III) was used as counter redox couple. Duplicate runs showed stable performance during 15 days, with acetate being the main energy carrier. An energy density of around $0.1 \mathrm{kWh} / \mathrm{m}^{3}$ (normalized to anode electrolyte volume) was achieved at full-cycle energy efficiency of 30 to $40 \%$, with a nominal power output during discharge of $190 \mathrm{~W} / \mathrm{m}^{3}$ (normalized to anode volume). The work presented shows a new potential application area for bioelectrochemical systems as a future local energy storage device. 


\subsection{INTRODUCTION}

With ever increasing worldwide energy demands and raised concerns about the environmental impacts of burning fossil fuels, renewable energy sources are slowly but steadily gaining ground ${ }^{[1]}$. One of the major challenges for implementing renewable electricity is the variability in generation of sun and wind energy and matching this with a fluctuating demand. Models predict that, as long as shares of renewables remain below $30 \%$ of the total electricity supplied to the grid, through smart grid technologies, dynamics in power grid demand-supply might be balanced without additional energy storage capacities ${ }^{[2]}$. However, energy storage devices will likely become a necessity with further increasing renewable electricity shares. Conventional energy storage systems like lithium batteries, compressed air energy storage (CAES), pumped hydro and newer technologies as sodium ion batteries will likely play their role to this end. Current storage systems often cope with safety issues (CAES) or toxicities (heavy metals) allowing use only under precisely controlled conditions, require scarce and non-renewable materials, or can be only used in suitable geographic environments (pumped hydro ${ }^{[3]}$. Therefore, a safe, renewable and low-cost system for household-scale energy storage would bear high potential. Bioelectrochemical systems (BESs) could play an important role in future energy storage, as the catalysts in these systems (i.e. microorganisms) (re)generate and use renewable and widely available substrates, namely water, $\mathrm{CO}_{2}$ and nutrients.

While both microbial electrosynthesis (MES) and microbial fuel cells (MFCs) have been subject of intensive study over the last decades, ${ }^{[4-9]}$ they have, to the authors' best knowledge, not yet been integrated into one system, with the objective to store and recover electricity. For this new concept, we introduce the name Microbial Rechargeable Battery (MRB). In a MRB, during the MES phase, electrical energy is consumed to form acetate, while during the MFC phase, electrical energy is generated by consumption of acetate. The proposed system would therefore require stable intermittent operation of both biocathode, bioanode, and their counter electrodes.

To provide a proof-of-concept of the MRB, we connected an acetate producing biocathode (MES) hydraulically to an acetate oxidizing bioanode (MFC) and operated both in turn with a total charge/discharge cycle period of 24 hours (thus matching day/night rhythm typical for solar energy production). During these cycles, $\mathrm{CO}_{2}$ was successfully converted into acetate and energy was recovered by subsequent oxidation of the acetate formed (see Figure 1 for a schematic overview). The ferri/ferrocyanide redox couple was used as reversible reaction at the counter electrodes. We analyzed the performance of the MRB in terms of efficiency and stability. 


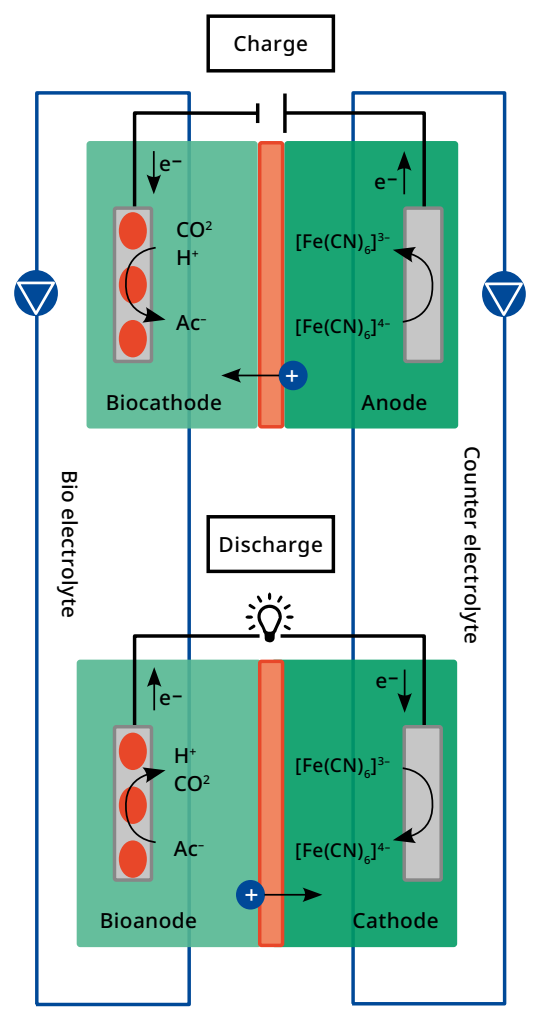

FIGURE 1 | Schematic overview of the Microbial Rechargeable Battery (MRB) consisting of a $\mathrm{CO}_{2}$ reducing charging cell (MES, upper) and an acetate oxidizing discharging cell (MFC, lower). Depicted are the (simplified) predominant reactions taking place at the bioanode, biocathode and their counter electrodes, flow of electrons (e-) across the external electrical circuits and the transfer of cations (+) across the membranes.

\subsection{MATERIALS AND METHODS}

\subsubsection{System design}

Experiments were conducted in duplicate setups, each consisting of two electrochemical cells. One set of cells is further referred to as a microbial rechargeable battery (MRB). For each MRB, one cell (charging cell) performed MES with an acetate producing biocathode, while the second (discharging cell) was operated as a microbial fuel cell (MFC) with an acetate consuming bioanode.

Each cell consisted of two Plexiglas flow compartments $\left(33 \mathrm{~cm}^{3}\right)$, one flat plate current collector (stainless steel SS316), a flat plate counter current collector ( $\mathrm{Pt} / \mathrm{lrO}_{2}$ coated Ti, Magneto special anodes BV, Schiedam, the Netherlands) and two support plates $^{[10]}$. Electrolytes were separated by a cation exchange membrane (Fumasep FKB-PEEK, Fumatech, projected surface area of $22 \mathrm{~cm}^{2}$ ). Both bioanode, biocathode 
and their counter electrodes consisted of plain graphite paper and 5 layers of graphite felt (thickness $3 \mathrm{~mm}$, FMI Composites Ltd., Scotland) firmly held between current collector and membrane, completely filling the flow compartments.

\subsubsection{Media and Microbial Inoculum}

The electrolyte, which was shared by both bioanode and biocathode and is further referred to as the bioelectrolyte, consisted of: $0.4 \mathrm{~g} / \mathrm{L} \mathrm{NH}_{4} \mathrm{HCO}_{3}, 0.05 \mathrm{~g} / \mathrm{L} \mathrm{Ca}(\mathrm{OH})_{2}, 0.1$ $\mathrm{g} / \mathrm{L} \mathrm{MgSO}_{4} \cdot 7 \mathrm{H}_{2} \mathrm{O}, 9.6 \mathrm{~g} / \mathrm{L} \mathrm{K}_{2} \mathrm{HPO}_{4}, 2.1 \mathrm{~g} / \mathrm{L} \mathrm{Na}-2-\mathrm{BES}, 4 \mathrm{~g} / \mathrm{L} \mathrm{NaOH}, 0.1 \mathrm{~mL} / \mathrm{L}$ trace metals ${ }^{[11]}$ and $0.1 \mathrm{~mL} / \mathrm{L}$ vitamins (DSMZ medium 141). At start, influent mineral medium was sparged with $\mathrm{CO}_{2}$ with a resulting initial $\mathrm{pH}$ of 6.4. Counter electrolyte was composed of $2.9 \mathrm{~g} / \mathrm{L} \mathrm{Na}_{4}\left[\mathrm{Fe}(\mathrm{CN})_{6}\right] \cdot 10 \mathrm{H}_{2} \mathrm{O}, 21 \mathrm{~g} / \mathrm{L} \mathrm{K}_{4}\left[\mathrm{Fe}(\mathrm{CN})_{6}\right] \cdot 3 \mathrm{H}_{2} \mathrm{O}, 4.0 \mathrm{~g} / \mathrm{L} \mathrm{K}{ }_{3}\left[\mathrm{Fe}(\mathrm{CN})_{6}\right], 1.7 \mathrm{~g} / \mathrm{L}$ $\mathrm{K}_{2} \mathrm{HPO}_{4}$ and was kept saturated with $\mathrm{CO}_{2}$ throughout the experiments. Bioelectrolyte and counter electrolyte conductivity was measured at start to be $1.6 \mathrm{~S} / \mathrm{m}$ and 2.9 $\mathrm{S} / \mathrm{m}$, respectively, thus not limiting current production throughout the experiment.

The bioelectrolyte was inoculated with a combination of (1) effluent from an acetate producing biocathode (inoculated previously with a mixed culture extracted from an anaerobic digester and cow manure) and (2) effluent from an active MEC running on acetate.

\subsubsection{Reactor start-up and operation}

Reference electrodes (Ag/AgCl, Prosense, Oosterhout, Netherlands, + $0.203 \mathrm{~V}$ vs. SHE) were connected to the electrolytes. All reported potentials were expressed relative to this reference. Current and power densities are reported normalized to bioelectrode volume $\left(33 \mathrm{~cm}^{3}\right)$ or membrane surface area $\left(22 \mathrm{~cm}^{2}\right)$. Energy and charge densities are normalized to bioelectrolyte recirculation volume $(280 \mathrm{~mL})$. Total counter electrolyte recirculation volume was $2240 \mathrm{~mL}$. All electrolytes were recirculated with $10 \mathrm{~mL} / \mathrm{min}$ pump speed. Bioelectrolyte $\mathrm{pH}$ was measured in-line (Endress+Hauser, CP571D-7BV21). Reactor temperature was maintained at (32 \pm 1) ${ }^{\circ} \mathrm{C}$ using climate control of the research cabinet. A multi-channel potentiostat (N-stat DC, Ivium Technologies, Eindhoven, Netherlands) was used to perform electrochemical measurements and experiments. Individual cell voltage and membrane potentials were measured using a data logger (RSG40, Endress+Hauser, Germany) in conjunction with high-impedance potentiometers.

Start-up of bioanode and biocathode occurred after inoculation and with continuous supply of medium. Bioanode potentials were controlled at -0.35 V. Biocathodes were current controlled at $-750 \mathrm{~A} / \mathrm{m}^{3}\left(-11.3 \mathrm{~A} / \mathrm{m}^{2}\right)$. After stable bioanodic currents were established and maintained for several days (4 to 6 days), the charge/discharge experiment was started. During the experiment the 
two cells of a MRB were switched in turns between open-circuit and controlled current over a total cycle period of 24 hours. First, a constant current of $-150 \mathrm{~A} / \mathrm{m}^{3}$ $\left(-2.26 \mathrm{~A} / \mathrm{m}^{2)}\right.$ was supplied to the biocathode (MES) for 16 hours (charging), during which the bioanode (MFC) was put in open-circuit conditions. The charging period was then followed by 8 hours of discharge with the bioanode current controlled at $300 \mathrm{~A} / \mathrm{m}^{3}\left(4.52 \mathrm{~A} / \mathrm{m}^{2}\right)$ and the biocathode put in open-circuit conditions in turn. During discharge, bioanode current was maintained until its potential reached $-0.35 \mathrm{~V}$, after which the potentiostat was programmed to switch to potential control to maintain this potential. This switch from galvanostatic to potentiostatic operation prevented unintended side reactions from taking place after acetate had depleted.

\subsubsection{Chemical Analysis and performance calculations}

Liquid samples of bioelectrolytes were taken $15 \mathrm{~min}$ prior to the end of each charging and discharging phase. During sampling, sample volumes of $5 \mathrm{~mL}$ were replaced by an equal volume of new $\mathrm{CO}_{2}$ saturated bioelectrolyte, keeping total recirculation volume constant. The samples were analyzed for fatty acid content (Dionex UHPLC System). Gas formation was quantified using a gas counter (MGC, Ritter Apparatebau, Germany), and headspace composition was analyzed through gas chromatography ( $\mu \mathrm{GC}$, Varian $\mathrm{CP} 4900$ ). For a more detailed description of analytical methods see Sousa (2015). ${ }^{12}$

Overall Coulombic Efficiency (Overall $\mathrm{CE}$, or $\mathrm{CE}_{\text {total }}$ ) represents total charge recovered $\left(\mathrm{Q}_{\mathrm{r}}\right)$ during the discharging period, compared to total charge used during the charging period $\left(Q_{c}\right)$ :

$$
C E_{\text {total }}=\frac{Q_{r}}{Q_{c}}
$$

The maximum fraction of charge recovered through intermediate storage in acetate was then calculated by:

$$
f_{Q, \text { acetate }}=\frac{\Delta c_{\text {,acetate }} V n F}{Q_{r}}
$$

With $\Delta \mathrm{c}_{\text {acetate }}$ being the measured concentration difference in acetate between charged and discharged electrolyte, $\mathrm{V}$ the effective electrolyte volume, $n$ the amount of electrons involved in acetate oxidation (8) and $F$ the Faraday constant.

The energy efficiency per cycle (EE) was calculated by dividing the integral of power over the discharging period (16 to $24 \mathrm{~h}$ ) by the integral of power over the charging period ( 0 to $16 \mathrm{~h}$ ):

$$
E E=\frac{\int_{16}^{24} P_{\text {discharge }}(t) d t}{\int_{0}^{16} P_{\text {charge }}(t) d t}
$$




\subsection{RESULTS AND DISCUSSION}

For each MRB, the charging cell performed MES with an acetate producing biocathode, while the discharging cell was operated as a MFC with an acetate oxidizing bioanode. Each cycle started with a charging period of 16 hours, during which biocathodes were controlled at $-5 \mathrm{~mA}\left(2.26 \mathrm{~A} / \mathrm{m}^{2}\right)$. In this period, the potential of both biocathodes was between $-0.9 \mathrm{~V}$ and $-1.0 \mathrm{~V}$; a value typical for the formation of hydrogen on graphite. ${ }^{[7]}$ Using ferrocyanide $\left(\mathrm{Fe}^{2+}\right)$ as electron donor, this required charging cell voltages of $-1.1 \mathrm{~V}$ to $-1.2 \mathrm{~V}$. Data for one representative cycle for each MRB is shown in Figure 2, which first shows the applied charging/discharging currents (Figure 2a), followed by the observed cell voltages and electrode potentials (Figure $2 \mathbf{b}$ ) and resulting power densities (Figure 2c). For a more detailed analysis of all electrode potentials, see Figure S1 in the appendix. During charging, bioanodes were operated under open circuit condition, with their open circuit potential (OCP) decreasing towards $-0.5 \mathrm{~V}$ to $-0.55 \mathrm{~V}$. After 16 hours, biocathodes were switched to open circuit and bioanodes were operated as MFC at constant current of $10 \mathrm{~mA}\left(4.52 \mathrm{~A} / \mathrm{m}^{2}\right)$. This resulted in anode potentials of $-0.46 \mathrm{~V}$ to $-0.43 \mathrm{~V}$ and discharge cell voltages of $+0.5 \mathrm{~V}$ to $+0.6 \mathrm{~V}$, corresponding to a power density of $2.8 \mathrm{~W} / \mathrm{m}^{2}$ to $3.0 \mathrm{~W} / \mathrm{m}^{2}$ throughout the first few hours of the discharge period. After a few hours, anode potentials showed a marked increase, indicating substrate depletion. When anode potential rose to values higher than $-0.35 \mathrm{~V}$, the potentiostat switched from current control to anode potential control $(-0.35 \mathrm{~V})$ and current decreased rapidly. After 8 hours of discharging, at which point current densities from the bioanodes had dropped to values $<0.5 \mathrm{~A} / \mathrm{m}^{2}$, a new cycle was started. Figure 2 (a and c) also shows the charging capacity $\left(\mathrm{MC} / \mathrm{m}^{3}\right)$ and energy density $(\mathrm{kWh} /$ $\mathrm{m}^{3}$ ) during both charge and discharge, represented by the shaded surface areas, from which overall Coulombic efficiency (CE) and energy efficiency was calculated. Cycles were repeated in a stable way during 15 days, illustrating the ability of biocathodes and bioanodes to become active directly after 8 to 16 hours of inactivity (open circuit) and to be operated intermittently throughout many cycles. No $\mathrm{pH}$ adjustments were done throughout the experiments, and only minor $\mathrm{pH}$ fluctuations were detected throughout the charge/discharge cycles due to presence of bicarbonate/carbonic acid and phosphate acting as a buffer. Despite the minor fluctuations in $\mathrm{pH}$, no net gas production was observed both during charging and discharging periods in general, indicating inorganic carbon stayed dissolved throughout the experiments. More detailed analysis of $\mathrm{pH}$ is provided in the appendix (Figure S2). 

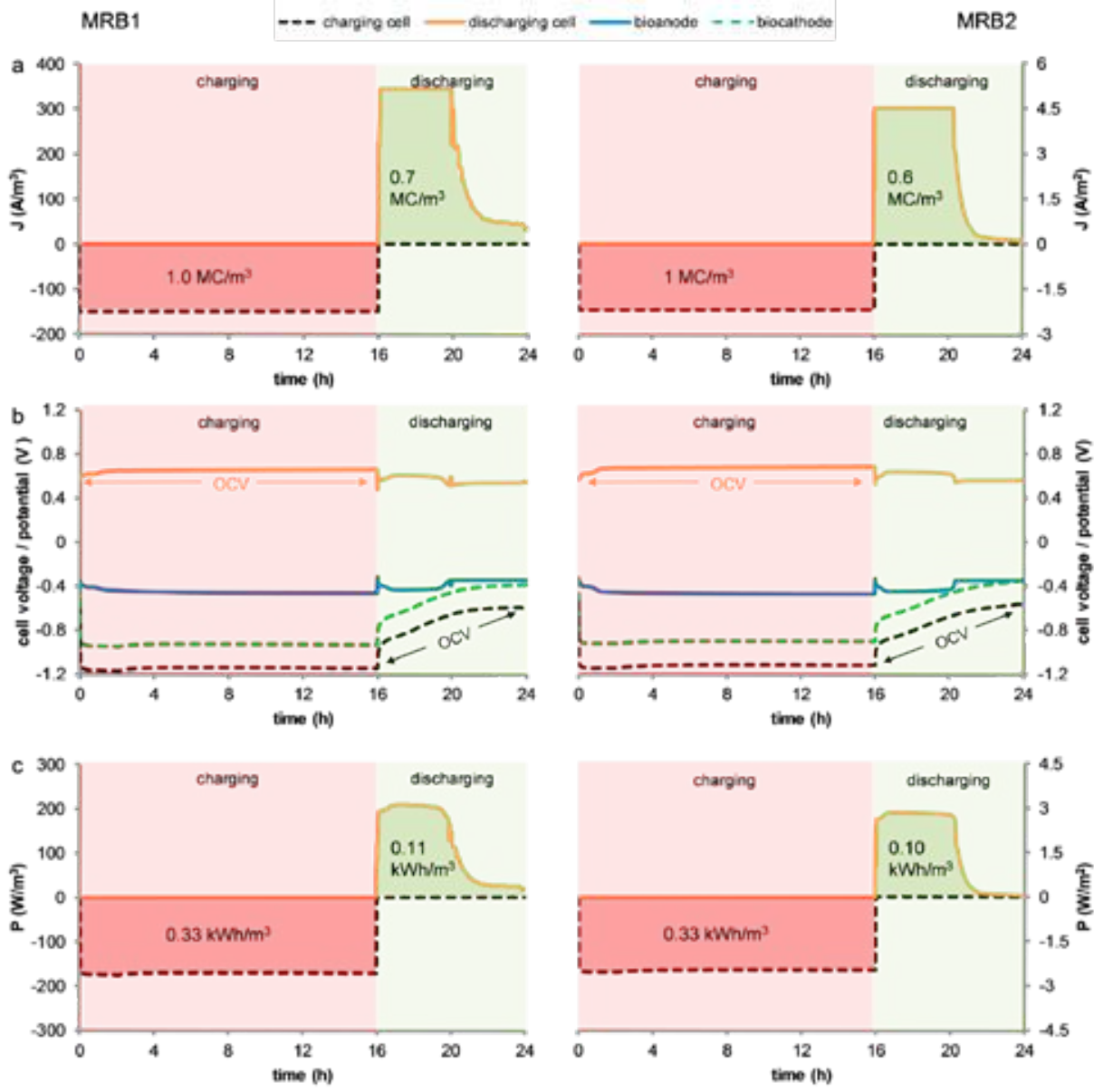

FIGURE 2 | Voltages/potentials (b) and (a) current and (c) power densities as typically observed throughout one cycle for both MRBs. Current and power densities are normalized to both total bioelectrolyte volume (left y-axis) and projected (membrane) surface area (right y-axis). As patterns throughout cycles showed great similarity, data of the last recorded cycle (no. 15) is depicted. This graph clearly shows the feasibility of a MRB based on acetate/carbonate redox chemistry.

Figure 3 shows the charge recovery efficiency for both MRBs, during each cycle of the experiment. Overall CEs, representing the electrons recovered from the bioanode compared to the electrons fed to the biocathode in one cycle, reached 50 to $80 \%$ throughout all cycles except one. Chemical analysis on liquid samples taken at the end of the charging and discharging period showed high selectivity towards acetate as product of $\mathrm{CO}_{2}$ reduction, and contribution of acetate to total stored charge was determined. Production of acetate was slightly lower in the first cycles, 

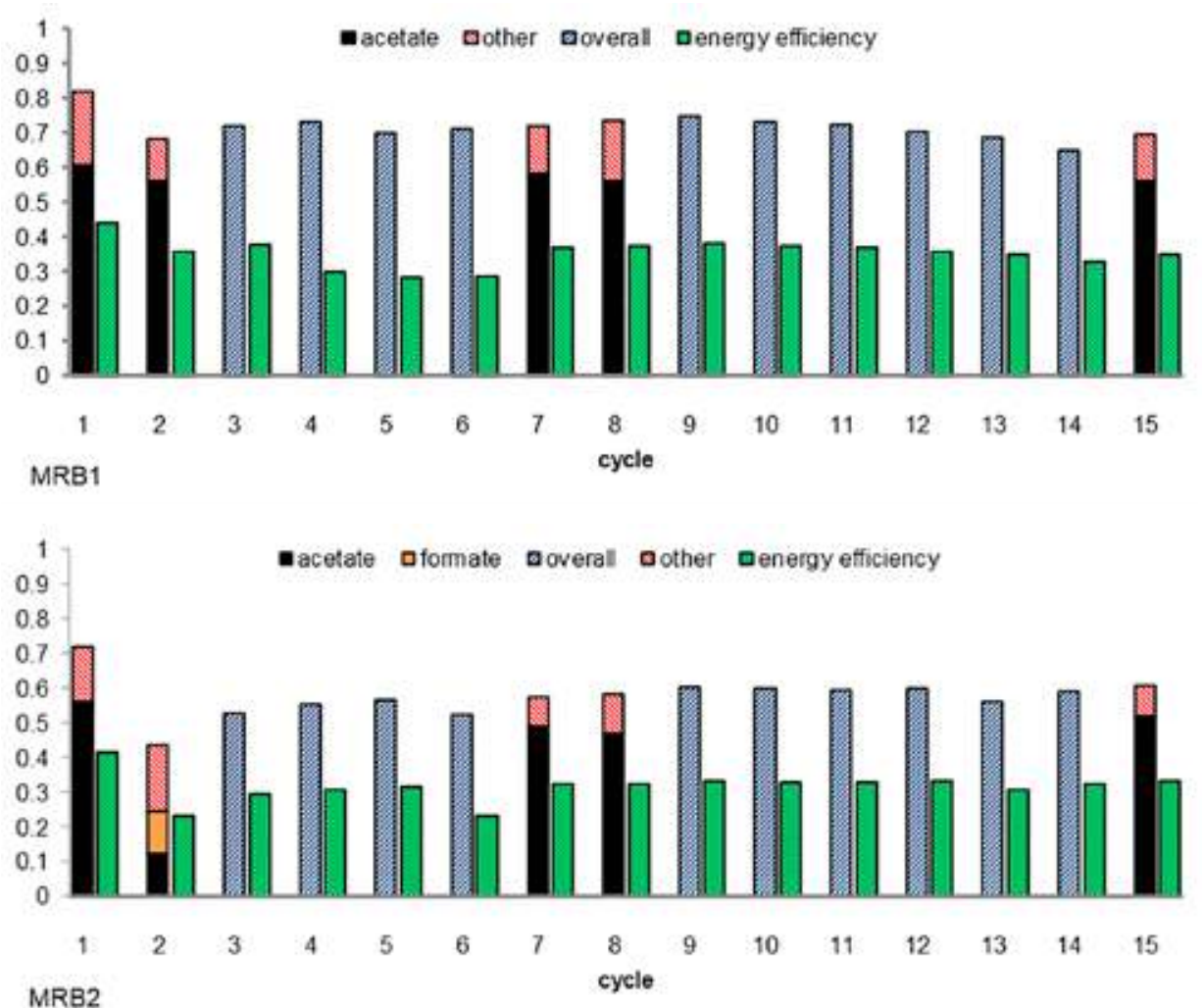

FIGURE 3 | Energy efficiency (green) and overall CE (full bar) throughout charge/discharge cycles for both microbial rechargeable batteries (MRB1\&2). Fractionation between charge recovered through acetate or other (charge carriers) was calculated from measured fluctuations in acetate concentration, assuming $100 \%$ anodic conversion efficiency of acetate to current. For cycle 3-6 and 9-14, no chemical analysis of the electrolytes was performed, thus for these cycles overall CE is displayed without further fractionation.

gradually increasing and then stabilizing in later cycles. Typically, 50 to $60 \%$ of electrons supplied to the biocathode during charging were stored in acetate, corresponding to final acetate concentrations of $0.68 \mathrm{mM}$ and $0.85 \mathrm{mM}$ respectively.

An exception to above general observations was formed by cycle 2 of MRB2, in which charge recovered in acetate (12\%) was lower. Remarkable for this cycle was production of formate, which in turn accounted for about $12 \%$ of transferred charge. The produced formate was fully degraded during the subsequent discharge, thus not contributing to losses to overall CE for this cycle. Moreover, during this cycle, measurable amounts of gas were produced, mainly consisting of hydrogen. This way, hydrogen losses accounted for lower CE during this cycle. Assuming the solution was saturated with hydrogen at the end of this specific cycle 
( $\pm 0.7 \mathrm{mM} \mathrm{H}_{2}$ ), dissolved hydrogen could account for maximum $4.5 \%$ of charge transfer from biocathode to bioanode. In subsequent cycles, these deviations from previous cycles did not reoccur, and mechanisms causing the disturbance in this single cycle were not further investigated.

As overall charge recovered exceeded charge stored in acetate in all cycles, presence of charge carriers other than acetate (as occurring in the bulk electrolyte) was imperative. Headspace hydrogen partial pressures typically reached $3 \%$ at full charge, with remaining part composed of $30 \% \mathrm{CO}_{2}$ and $66 \% \mathrm{~N}_{2}$ (no methane detected). No net production of gas was observed during cycles other than discussed previously and the low partial pressure of hydrogen and accompanying dissolved hydrogen concentration was not high enough to contribute substantially to the total charge stored. However, it seems plausible hydrogen was intermediate in acetate formation, with the biocathode enabling almost full conversion of the produced hydrogen to acetate, in accordance with recent findings ${ }^{[11]}$. SEM examination of the electrode material showed only limited presence of bacteria attached to the biocathodes, while bioanodes possessed well developed biofilms (Figure S3). Microbial characterization of the obtained biomass was not further pursued at this stage.

An alternative charge carrier, besides $\mathrm{H}_{2}$ and acetate, could be other organics, or inorganics like sulphate. The role of sulphate was likely limited, as measured sulphate concentrations showed no substantial fluctuations throughout the cycles (data not shown). Alternatively, the unexplained charge recovered could be stored in the anodic biofilm instead of in the bulk electrolyte. Possibly, electrons are transferred to and accumulating in or at the anodic biofilm during the charging phase. The mediator responsible for this electron transfer from biocathode to bioanode can be any biologically available redox active compound. The anodic biofilm is presumed to possess a pool of redox mediators in its intercellular environment which, during charging, is gradually reduced while under open circuit conditions ${ }^{[13,14]}$. The extent to which this mechanism could play a role was not further investigated, and will become less evident when the system is operated at higher energy densities (thus reaching higher acetate concentrations).

In the current study, specific energy densities around $0.1 \mathrm{kWh} / \mathrm{m}^{3}$ were reached. In order for the proposed technology to be competitive with conventional batteries, this energy density needs to be increased. A first important factor impacting energy density is the choice of counter electrode. Here, the counter electrode reaction, using hexacyanoferrate, was selected for practical reasons (soluble at neutral $\mathrm{pH}$, reversible reaction and reasonably high potential). A next step is to find a suitable, reversible and environmentally attractive counter electrode reaction to further demonstrate the feasibility of the MRB. Finding a better counter electrode reaction has another potential advantage: overall energy efficiency $(\mathrm{n}=30)$ was on average $33.5 \%(\mathrm{~s}=4.5 \%)$. While part of the energy efficiency loss was explained by Coulombic losses (on average $35 \%, s=9 \%, n=30$ ), 
the main part was due to voltage losses. These voltage losses are to some extent inevitable as long as hydrogen is required as intermediate for acetate production, requiring considerable overpotential during charging compared to the obtained anode potential during discharging. By selecting a counter redox reaction with a sufficiently high redox potential, the relative difference between the required charging voltage and the obtained discharging voltage may be reduced, positively impacting overall energy efficiency.

Apart from the reaction at the counter electrode, the maximum achievable energy density of the MRB is directly related to acetate concentration attained during charging as this defines the anodes' charge capacity. Acetate concentrations of $0.75 \mathrm{M}$ reached previously in hydrogenotrophic reactors provide an optimistic perspective $^{[15]}$ regarding further optimization of this parameter.

To conclude, we have shown here the proof-of-concept of a Microbial Rechargeable Battery, using a biocathode that produces acetate from electricity, and a bioanode converting acetate into electricity. Depending on the acetate concentration that can be achieved, and the counter reaction involved, the MRB could become a suitable, clean, safe and renewable alternative to existing battery storage systems. As such, the MRB could become an inexpensive local energy storage device in the future.

\subsection{ACKNOWLEDGEMENTS}

This work was performed in the cooperation framework of Wetsus, European Centre of Excellence for Sustainable Water Technology (www.wetsus.eu). Wetsus is co-funded by the Dutch Ministry of Economic Affairs and Ministry of Infrastructure and Environment, the Province of Fryslân, and the Northern Netherlands Provinces. The authors like to thank the participants of the Resource Recovery research theme for the fruitful discussions and their financial support. 


\subsection{REFERENCES}

(1) Wüstenhagen, R.; Menichetti, E. Strategic Choices for Renewable Energy Investment: Conceptual Framework and Opportunities for Further Research. Energy Policy 2012, 40 (1), 1-10.

(2) Denholm, P.; Ela, E.; Kirby, B.; Milligan, M. The Role of Energy Storage with Renewable Electricity Generation. Natl. Renew. Energy Lab. 2010, 1-61.

(3) Dunn, B.; Kamath, H.; Tarascon, J.-M. Electrical Energy Storage for the Grid: A Battery of Choices. Science 2011, 334 (6058), 928-935.

(4) Sleutels, T. H. J. A.; Ter Heijne, A.; Buisman, C. J. N.; Hamelers, H. V. M. Bioelectrochemical Systems: An Outlook for Practical Applications. ChemSusChem 2012, 5 (6), 1012-1019.

(5) Harnisch, F.; Schröder, U. From MFC to MXC: Chemical and Biological Cathodes and Their Potential for Microbial Bioelectrochemical Systems. Chem. Soc. Rev. 2010, 39 (11), 4433-4448.

(6) Nevin, K. P.; Woodard, T. L.; Franks, A. E. Microbial Electrosynthesis : Feeding Microbial Electrosynthesis : Feeding Microbes Electricity To Convert Carbon Dioxide and Water to Multicarbon Extracellular Organic. Am. Soc. Microbiol. 2010, 1 (2), 1-4.

(7) Jourdin, L.; Grieger, T.; Monetti, J.; Flexer, V.; Freguia, S.; Lu, Y.; Chen, J.; Romano, M.; Wallace, G. G.; Keller, J. High Acetic Acid Production Rate Obtained by Microbial Electrosynthesis from Carbon Dioxide. Environ. Sci. Technol. 2015, 49 (22), 13566-13574.

(8) Gildemyn, S.; Verbeeck, K.; Slabbinck, R.; Andersen, S. J.; Prévoteau, A.; Rabaey, K. Integrated Production, Extraction, and Concentration of Acetic Acid from CO 2 through Microbial Electrosynthesis. Environ. Sci. Technol. Lett. 2015, 2 (11), 325-328.

(9) LaBelle, E. V.; Marshall, C. W.; Gilbert, J. A.; May, H. D. Influence of Acidic PH on Hydrogen and Acetate Production by an Electrosynthetic Microbiome. PLoS One 2014, 9 (10), e109935.

(10) ter Heijne, A.; Hamelers, H. V. M.; Saakes, M.; Buisman, C. J. N. Performance of Non-Porous Graphite and Titanium-Based Anodes in Microbial Fuel Cells. Electrochim. Acta 2008, 53 (18), 5697-5703.

(11) Zehnder, A. J. B.; Huser, B. A.; Brock, T. D.; Wuhrmann, K. Characterization of an AcetateDecarboxylating, Non-Hydrogen-Oxidizing Methane Bacterium. Arch. Microbiol. 1980, 124 (1), 1-11.

(12) Sousa, J. A. B.; Plugge, C. M.; Stams, A. J. M.; Bijmans, M. F. M. Sulfate Reduction in a Hydrogen Fed Bioreactor Operated at Haloalkaline Conditions. Water Res. 2015, 68 (0), 67-76.

(13) Uría, N.; Muñoz Berbel, X.; Sánchez, O.; Muñoz, F. X.; Mas, J. Transient Storage of Electrical Charge in Biofilms of Shewanella Oneidensis MR-1 Growing in a Microbial Fuel Cell. Environ. Sci. Technol. 2011, 45 (23), 10250-10256.

(14) Freguia, S.; Rabaey, K.; Yuan, Z.; Keller, J. Electron and Carbon Balances in Microbial Fuel Cells Reveal Temporary Bacterial Storage Behavior during Electricity Generation. Environ. Sci. Technol. 2007, 41 (8), 2915-2921.

(15) Demler, M.; Weuster-Botz, D. Reaction Engineering Analysis of Hydrogenotrophic Production of Acetic Acid by Acetobacterium Woodii. Biotechnol. Bioeng. 2011, 108 (2), 470-474. 


\subsection{APPENDICES}
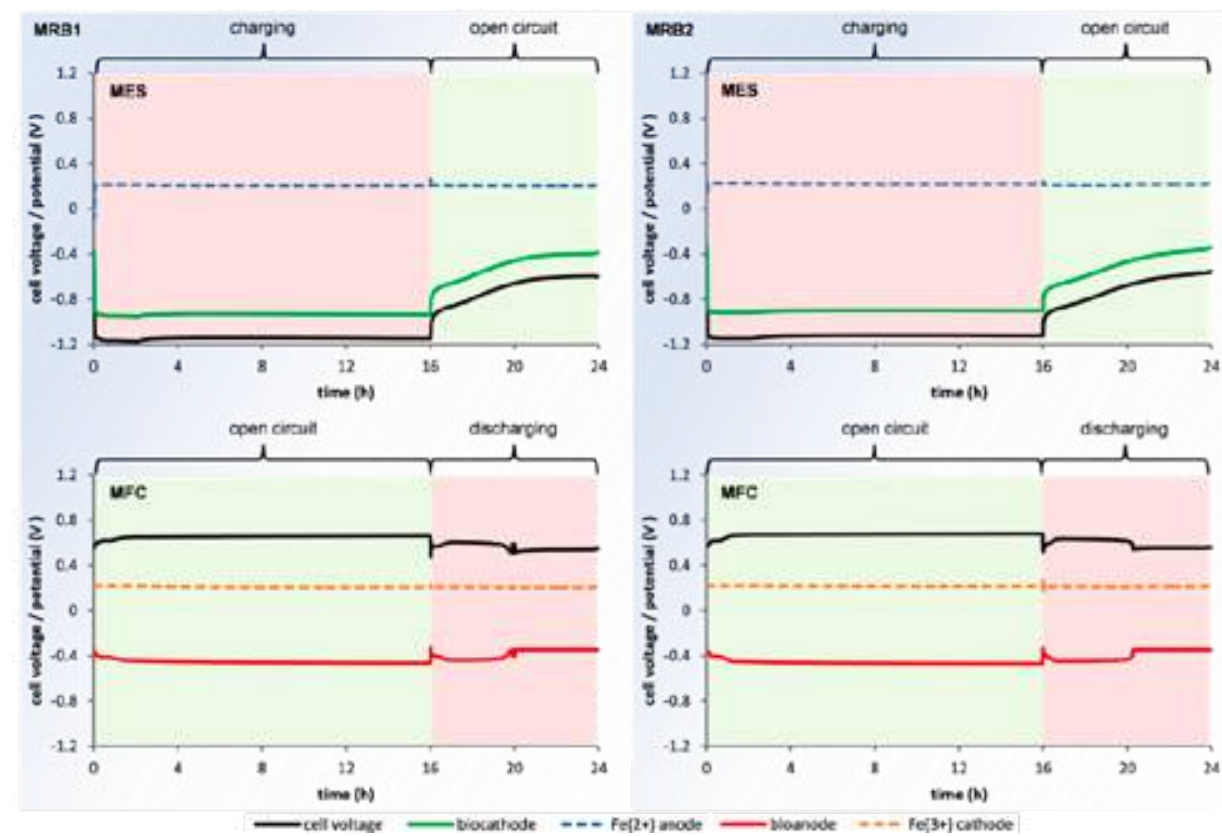

FIGURE S1 | Overview of MRB charging/discharging voltages and electrode potentials (vs. $\mathrm{Ag} / \mathrm{AgCl}$ ) as typically observed throughout one cycle. As patterns throughout cycles showed great similarity, data of the last recorded cycle (no. 15) is depicted. In the upper two graphs, the potential of the biocathode (green) and the counter anode $\left(\mathrm{Fe}^{2+} \rightarrow \mathrm{Fe}^{3+}\right.$,blue, dashed) is plotted along with the resulting negative cell voltage required for charging the system (black). In the lower two graphs, the bioanode potential (red) and the counter cathode ( $\mathrm{Fe}^{3+} \rightarrow \mathrm{Fe}^{2+}$ orange, dashed) is plotted along with the resulting positive cell voltage (black). The reversible nature of the hexacyanoferrate couple resulted in no observable overpotentials compared to its OCP during its use both as cathode or anode, thus creating almost horizontal lines in all plots. 


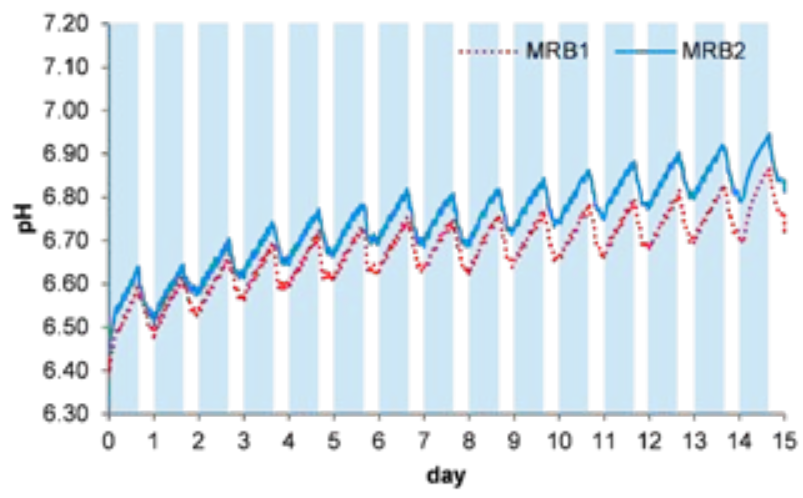

FIGURE S2 | Recorded pH values for both MRBs during charge/discharge periods. Blue shaded areas were charging periods (16 h) during which pH increased. On the subsequent discharge (non-shaded, 8 $\mathrm{h}$ period) the $\mathrm{pH}$ decreased again. Overall $\mathrm{pH}$ slightly increased over the cycles, most probably due to a net consumption of $\mathrm{CO}_{2}$ into non acidic biomass.

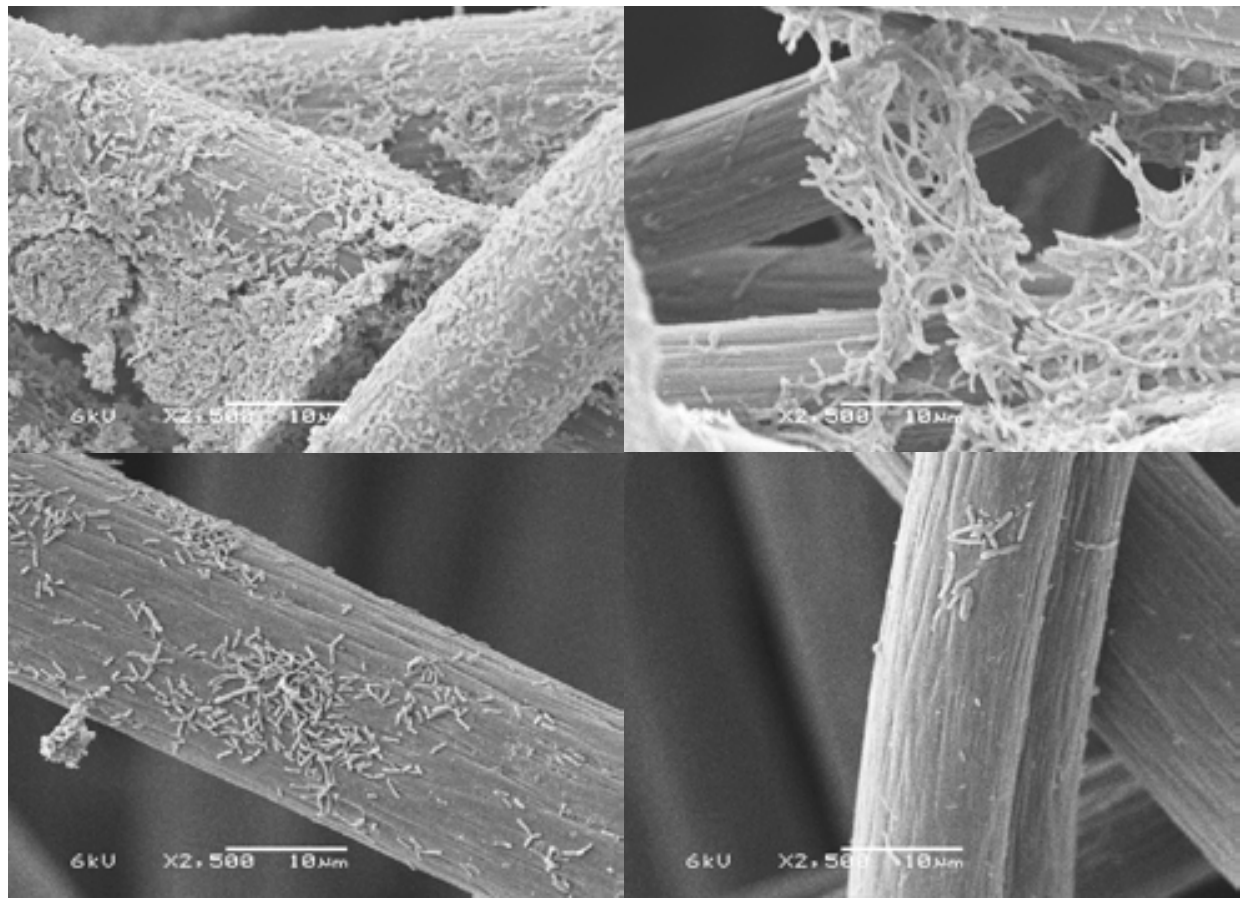

FIGURE S3 | SEM pictures taken from bioanode and biocathode graphite felt after the experiments had ended. Top left: MRB1 anode. Top right: MRB1 cathode. Bottom left: MRB2 anode. Bottom right: MRB2 cathode. All pictures were taken at 2500x magnification. In general, biofilm coverage was substantially higher on anodes than cathodes. Cathodes showed more loosely bound biomass with more EPS coverage, while anodes showed distinguishable cell morphology. 


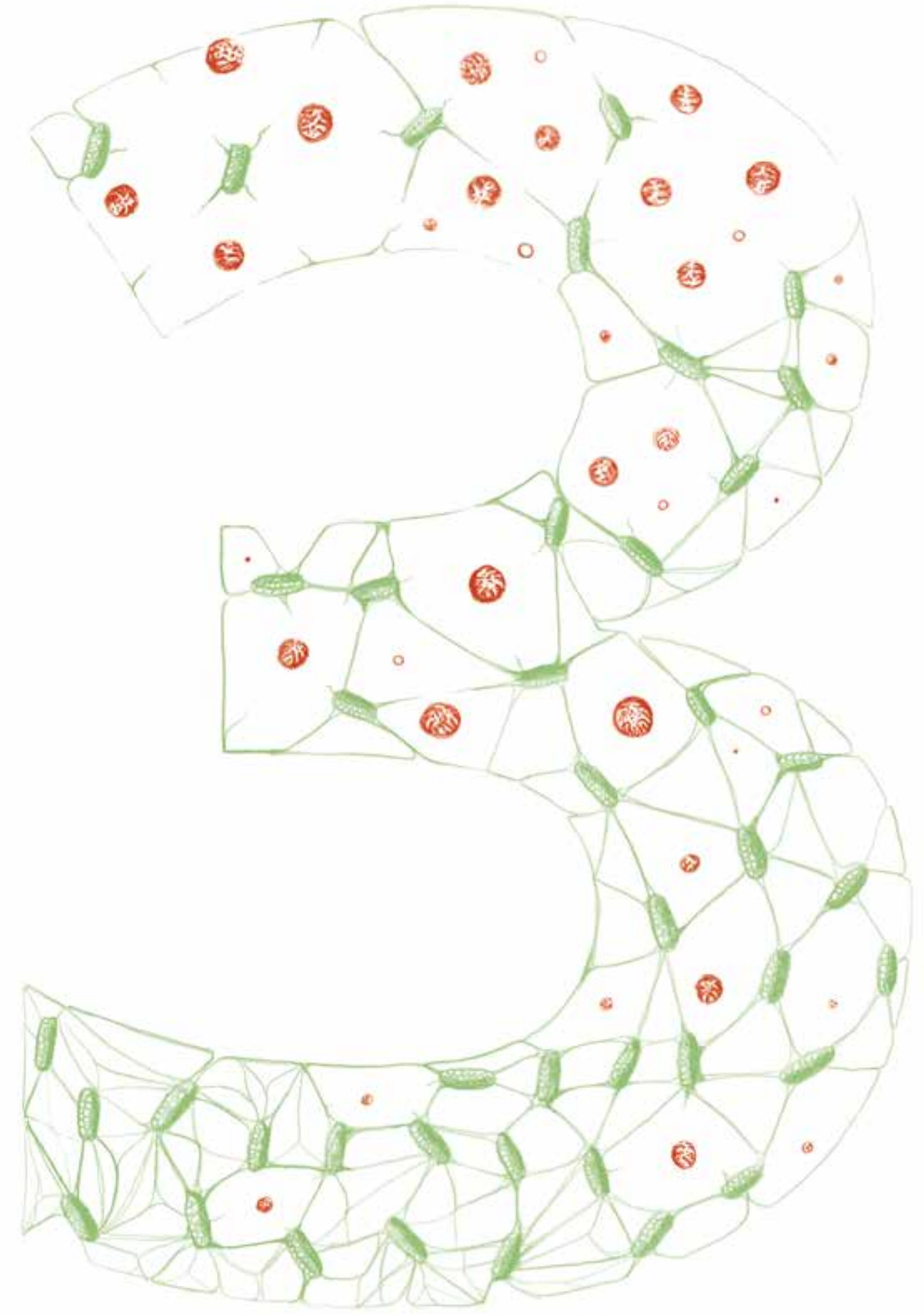




\section{LOW SUBSTRATE LOADING LIMITS METHANOGENESIS AND LEADS TO HIGH COULOMBIC EFFICIENCY IN BIOELECTRO- CHEMICAL SYSTEMS}

A crucial aspect for the application of bioelectrochemical systems (BESs), either as a wastewater treatment technology or as energy storage device, is the efficient oxidation of (complex) substrates by the bioanode, which is reflected in high Coulombic efficiency (CE). To achieve high CE, it is essential to give a competitive advantage to electrogens over methanogens. Factors that affect CE in bioanodes are, amongst others, the electrolyte composition, anode potential, substrate concentration and $\mathrm{pH}$. In this chapter, focus is put on acetate as a substrate and the competition between methanogens and electrogens is analyzed from a thermodynamic and kinetic point of view. Experimental data from earlier studies is reviewed and we propose that low substrate loading in combination with a sufficiently high anode overpotential plays a key-role in achieving high CE. Low substrate loading is a proven strategy against methanogenic activity in large-scale reactors for sulfate reduction. The combination of low substrate loading with sufficiently high overpotential is essential because it results in favorable growth kinetics of electrogens compared to methanogens. To achieve high current density in combination with low substrate concentrations, it is essential to have a high specific anode surface area. New reactor designs with these features are essential for BESs to be successful in wastewater treatment in the future, and the theory may also be applicable to other applications for BESs where closed microbial communities cannot be guaranteed throughout system operation. 


\subsection{INTRODUCTION}

In the past few decades, bioelectrochemical systems (BESs) have been mentioned as a promising technology for the conversion of chemical energy present in wastewaters directly into electrical energy ${ }^{[1]}$, using bioanodes as the driver for different types of cathodic reduction reactions. To this point, however, BESs have not successfully been applied to the full scale to be more efficient than more conventional state-of-the-art technologies, like anaerobic digestion ${ }^{[2]}$. As we will discuss below, a crucial aspect limiting this development is the efficient extraction of electrons from complex substrates.

Microbial fuel cells (MFCs) and microbial electrolysis cells (MECs) are two specific types of BESs. Similar to all other electrochemical systems, in BESs, the reduction and oxidation reactions are spatially separated, and their occurrence can be manipulated externally by means of an electrical circuit. Essentially different from other electrochemical systems is the use of electrochemically-active microorganisms, which can exchange electrons with an electrode. In MFCs and MECs, such electrochemically-active microorganisms (also called electrogens) are used to oxidize biodegradable (organic) components from wastewater and transfer the electrons to the anode. When combined with a suitable cathode, these systems comprise a promising technology for clean and efficient recovery of energy from wastewaters ${ }^{[1-3]}$. When a bioanode is coupled to a cathode where a reduction reaction occurs at a higher potential than the oxidation of the substrate, for example the reduction of oxygen, energy is recovered in the form of electricity, and the system is called a microbial fuel cell (MFC) ${ }^{[1]}$. When a bioanode is coupled to a cathode where a product is formed at a potential lower than the anodic reaction, an additional electrical energy input is required, and the system is called a microbial electrolysis cell (MEC) ${ }^{[3,4]}$. The understanding of bioanodes in BESs is crucial for the application in wastewater treatment. Promising applications for the use of bioanodes are, amongst others, for the recovery of energy and nutrients from urine $^{[5-7]}$, integration in anaerobic digestion to improve methane recovery ${ }^{[8,9]}$ and as the driver for different types of cathodic reduction reactions, like the production of hydrogen, but also caustic or hydrogen peroxide ${ }^{[10,11]}$.

Traditionally, MFCs serve two purposes at the same time: (1) to recover renewable energy in the form of electricity; and (2) to produce clean water that can be discharged to surface waters, by removing the biodegradable material. For MFCs, the performance is often analyzed in terms of anodic current density, which reflects the specific conversion rate, and power density, which reflects the rate at which energy is recovered. Besides these performance parameters, two types of efficiencies are used to evaluate MFC performance: (1) the Coulombic efficiency (CE); and (2) the voltage efficiency (VE). The CE shows the fraction of the electrons obtained from oxidizable substrates present in the wastewater, which 
are recovered at the anode, indicating the efficiency of the conversion of a substrate into electrical current. When a continuous system has reached equilibrium and no change over time of both influent and effluent concentrations and measured current occurs, the CE can be calculated with:

$$
C E_{\text {anode }}=\frac{I_{\text {measured }}}{n F \varphi\left(C_{\text {out }}-C_{\text {in }}\right)}
$$

where $I_{\text {measured }}$ is the measured current in A, $\mathrm{n}$ is the moles of electrons produced per mol of substrate, F is the Faraday constant $\left(96,485 \mathrm{C} \cdot \mathrm{mol}^{-1}\right), \varphi$ is the inflow rate of substrate $\left(\mathrm{m}^{3} \cdot \mathrm{s}^{-1}\right)$ and $\mathrm{C}_{\text {out }}$ and $\mathrm{C}_{\text {in }}$ are the substrate concentrations in the effluent and influent. Here, we only would like to consider continuously-operated systems, since for the treatment of large volumes of wastewater that are produced continuously, a reactor needs to be used that can be operated for an extended period of time.

The VE expresses the produced cell voltage $\left(E_{\text {cell }}\right)$ under load as a fraction of the equilibrium voltage at zero current $\left(\mathrm{E}_{\text {emf }}\right)$. The latter voltage can be calculated theoretically using the Nernst equation as described in, for example, Logan et al. $(2006)^{[1]}$. The VE is defined as:

$$
V E=\frac{E_{\text {cell }}}{E_{\text {emf }}}
$$

The overall energy efficiency is then calculated by multiplying the CE with the VE (Figure 1). With MFCs aiming for the production of electrical power directly, in one step from wastewater, these systems are often considered as a future alternative to anaerobic digestion (AD). AD produces methane instead of electricity and, as such, has received considerable scientific attention at the onset of BES research, because electricity is a more attractive decentral energy carrier. In view of energy recovery, an overall energy efficiency of $30 \%$ is a typical value reported to make MFCs competitive with anaerobic digestion ${ }^{[12]}$. Given the earlier definition of overall energy efficiency, it is clear that both CE and VE need to be optimized for MFCs to become an energy-efficient technology.

The optimization of VE has its boundaries: in order to obtain high VE, the observed cell voltage needs to be close to the open circuit voltage (OCV). Under such conditions of low overpotential, this means, in general, that current density is low. Conversely, when the anode potential is controlled at higher overpotential, higher current densities can be achieved ${ }^{[13]}$. However, controlling the anode potential at higher levels goes at the expense of the harvested cell voltage and, therefore, the VE.

In general, assuming constant internal resistance, an electrochemical system has $50 \%$ voltage efficiency at maximum power density. Therefore, for an MFC to be operated at maximum power density, the anode CE should be at least $60 \%$ in order to achieve an overall energetic efficiency of at least 30\% (Figure 1). 


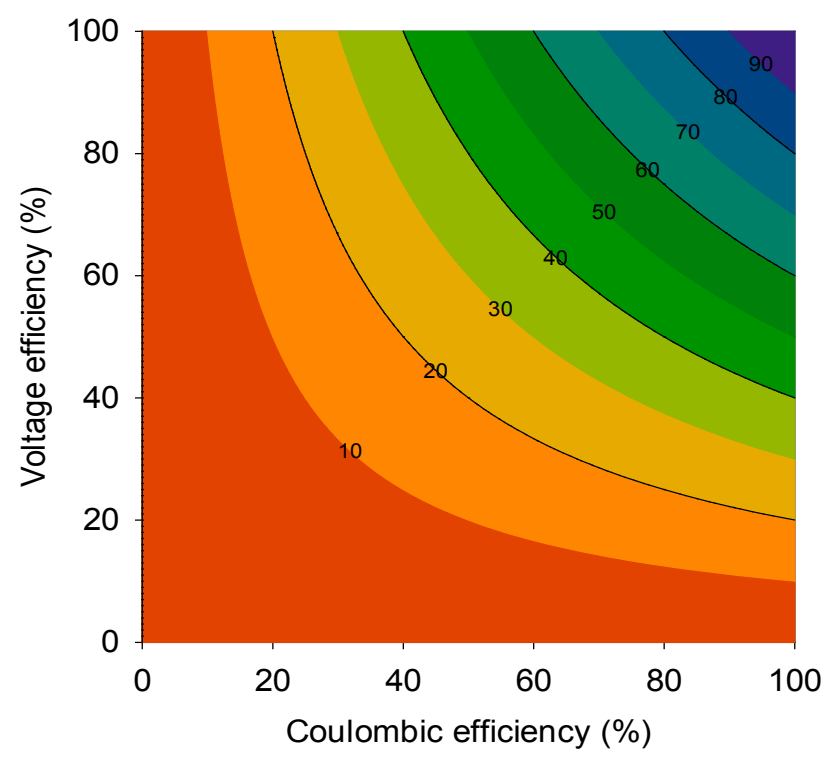

FIGURE 1 | The overall efficiency of a microbial fuel cell (MFC) (contour lines in figure) plotted as a function of the voltage efficiency (\%) and the Coulombic efficiency (\%). In terms of energy efficiency, to be competitive with anaerobic digestion, an overall energy efficiency of at least $30 \%$ should be achieved (adapted from Hamelers et $\mathrm{al}^{[12]}$ ).

It thus becomes evident that, in order to operate MFCs anywhere near their maximum power density, these systems rely on attaining appreciable anodic CEs for their overall functioning. This review summarizes the factors affecting CE, discusses how thermodynamics and kinetics affect the competition between electrogens and methanogens and gives an outlook for the prerequisites of BESs to become competitive with AD.

\subsection{MAIN FACTORS LEADING TO CE LOSSES}

The processes leading to losses in CE in BESs can be roughly divided into three main categories: (1) parasitic processes related to the presence of alternative electron acceptors; (2) fermentative processes; and (3) substrate consumption by biomass formation (carbon assimilation). To which extent these processes occur is determined by the Gibbs free energy and the kinetics of their underlying reactions. The consumption of substrate by biomass formation (Category 3) is imperative to the concept of a biocatalyzed electrode, as biomass needs to grow and maintain itself in order for the system to work. Thus, to some extent, CE losses due to biomass 
formation and maintenance are inevitable and may be kept to a minimum by a combination of operational parameters, as will be discussed later.

Regarding alternative electron acceptors (Category 1), nitrate and sulfate impose the most common threat to $\mathrm{CE}$ based on their frequent presence in wastewaters. With their standard reduction potentials being considerably higher than those commonly applied to anodes in BESs (Table 1), the Gibbs free energy available by substrate oxidation using these electron acceptors is higher than the Gibbs free energy for using the electrode as electron acceptor. These reactions are a property of the wastewater and can therefore not be controlled.

Wastewaters often contain a range of complex organic compounds, which can be fermented through a broad variety of fermentation pathways (Category 2), as reviewed by Agler et al. (2011) ${ }^{[15]}$. Fermentation reactions lead to the formation of shorter carbon chains out of longer ones and the formation of hydrogen and $\mathrm{CO}_{2}$. Most of these fermentation reactions have favorable available Gibbs free energies and/or reaction kinetics compared to anodic oxidation of complex organic compounds in BESs. When complex organic wastewaters are used as a substrate in BESs, fermentation processes will therefore dominate. On the one hand, fermentation reactions lead to VE losses, because fermentation products have higher redox potentials than their reactants, while on the other hand, fermentation reactions do not necessarily lead to CE losses, because the fermentation products, mainly acetate and hydrogen, can be oxidized at the bioanode. Pre-fermentation can even be regarded as an essential step in operating BESs fed with wastewater: so far, higher current densities in bioanodes are reached when using acetate, compared to complex organic wastewaters ${ }^{[16,17]}$.

A possible disadvantage of pre-fermentation for the operation of BESs is the formation of methane. As soon as methane is formed, it can be regarded as a permanent loss to current production and, thus, CE, because it takes away the substrate for the bioanode. Table 1 gives an overview of the redox potentials of

TABLE1| The oxidation reduction potential range (ORP) of common compounds present in wastewater ${ }^{[14]}$.

\begin{tabular}{|l|l|}
\hline Biochemical Activity & ORP Range $(\mathrm{mV})$ \\
\hline Aerobic carbon oxidation & +50 to +200 \\
Nitrification & +150 to +350 \\
Denitrification & -50 to +50 \\
Acidification & -40 to -200 \\
Sulfate reduction & -50 to -250 \\
Methanogenesis & -200 to -400 \\
\hline
\end{tabular}


different processes occurring in wastewater treatment. As the potential of methane formation ( -200 to $-400 \mathrm{mV} v s$. $\mathrm{Ag} / \mathrm{AgCl})$ is in the same range as anode potentials commonly applied in BESs, it becomes clear that methanogenesis will be the main competing process occurring at bioanodes. The crucial question to achieve high $\mathrm{CE}$ in BESs is therefore: how can methanogenesis be prevented?

\subsection{SUBSTRATE LOADING RATE AS A CONTROL PARAMETER FOR HIGH CE}

Steering mixed cultures towards the desired product by means of process engineering is a proven strategy in many biotechnological applications. To this end, there are lessons to be learned about competition with methanogens from large-scale continuous bioreactors. An example of such a large-scale process in which the prevention of methanogenesis is crucial is biological sulfate reduction, which occurs at redox conditions similar to bioanodes.

Biological sulfate reduction is essential for the treatment of wastewaters containing sulfate, e.g., in the chemical industry. The competition between sulfate reducers and methanogens has been studied in detail; see, e.g., Visser et al. ${ }^{[18]}$. Monod growth kinetics of sulfate reducers on acetate are less favorable than those of methanogens. Therefore, under standard conditions, a major part of the substrate would be lost to methanogenesis. However, when operated at low substrate concentrations, sulfate reducers are at an advantage, resulting in only small amounts of substrate lost to the formation of methane. Most electrons from the electron donor will flow to sulfate reducers as long as sulfate is above $200 \mathrm{mg} / \mathrm{L}$ and no excess amount of chemical oxygen demand (COD) is present ( $<200 \mathrm{mg} / \mathrm{L}$ ). In sulfate reducing reactors, the competition is therefore solely being controlled by the substrate loading of the system.

Electrogens, similar to sulfate reducers, use acetate as the electron donor, and therefore, similar competition strategies to keep out methanogens may be applicable for MFCs and biological sulfate reduction. Therefore, keeping substrate concentration low could be key to achieving high CE. An experimental indication of how methanogens and electrogens interact at a bioanode is shown in Figure 2. This figure shows an anodic biofilm growing on the fiber of a graphite felt electrode while fed with acetate ${ }^{[19]}$. "Based on visible differences in cell morphology, it may be hypothesized that a layer of currentproducing biofilm (small rod-shaped cells) is covered by a layer of methanogens (large spherical cells). Presuming this is correct, the outside of the biofilm is facing the bulk acetate concentration of the electrolyte and is therefore always experiencing the highest concentration of acetate available. This promotes the growth of methanogens. Obviously, the growth of a layer of methanogens on top 


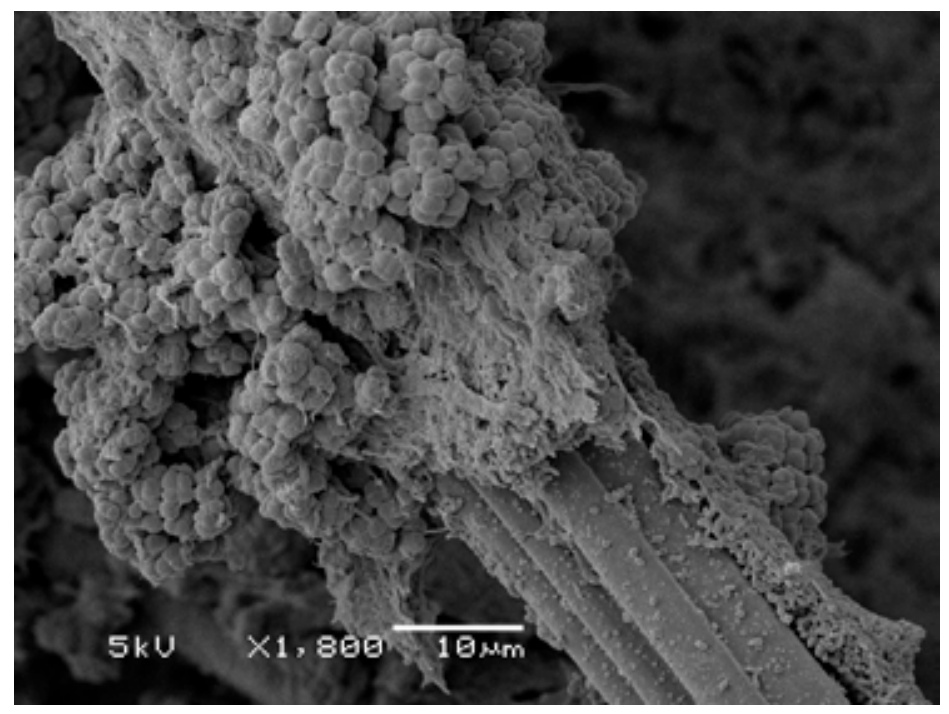

FIGURE 2 | Biofilm developed on the fibers of an anode felt electrode collected from a microbial electrolysis cell exhibiting measurable rate of methane production ${ }^{19}$. Based on morphological differences within the present biomass seen on the surface of the graphite fiber, it may be hypothesized methanogens (large, spherical cells) overgrow the electrogenic biofilm (small rod-shaped cells).

of electrogens would have a negative effect on CE, as the methanogens then have first access to the substrate. Giving electrogens a competitive advantage over methanogens from the start of operation is therefore a necessity for achieving high CE.

\subsection{ANODE OVERPOTENTIAL AS A CONTROL PARAMETER FOR HIGH COULOMBIC EFFICIENCY}

Whereas sulfate reducers use sulfate as the electron acceptor, electrogens use the electrode. Conversely, where in sulfate reduction, sufficiently high sulfate concentration is required to obtain high process selectivity, to control the competition with methanogens in the case of bioanodes, the anode overpotential may be used.

The overpotential is the difference between the equilibrium potential of acetate oxidation and the actual electrode potential. Representing the anode reaction's driving force, the overpotential is intricately linked to the reaction rate and Gibbs free energy available to the microorganisms. Next to a low substrate 
concentration, it provides an additional way to control the competition between microorganisms compared to conventional fermentations.

Only a few systematic studies have been performed that aim at determining the $\mathrm{CE}$ as a function of substrate concentration and overpotential. For example, Sleutels et al. (2011) showed that, to reach a CE over $80 \%$, a substrate effluent concentration lower than $1 \mathrm{mM}$ of acetate is required at an anode potential of $-350 \mathrm{mV} v s . \mathrm{Ag} / \mathrm{AgCl}$ or an anode potential higher than $-250 \mathrm{mV} v \mathrm{~s} . \mathrm{Ag} / \mathrm{AgCl}$ at an excess of acetate $(>20 \mathrm{mM})^{[20]}$. Of course, the exact numbers highly depend on the cell design and experimental conditions, for instance $\mathrm{pH}$ and mixing. In the following, we will discuss the fundamentals behind the competition with methanogens in more detail, by first analyzing the thermodynamics in relation to anode overpotential and, secondly, analyzing the growth kinetics of electrogens and methanogens.

First of all, the Gibbs free energy determines the energy available in the overall reaction.

Starting with acetate as the substrate, the oxidation reaction common to both methanogenesis, sulfate reduction and electrogenesis can be written as:

$$
\mathrm{CH}_{3} \mathrm{COO}^{-}+4 \mathrm{H}_{2} \mathrm{O} \rightarrow 2 \mathrm{HCO}_{3}^{-}+9 \mathrm{H}^{+}+8 \mathrm{e}^{-}
$$

For methanogenesis, the reduction reaction is:

$$
\mathrm{HCO}_{3}{ }^{-}+9 \mathrm{H}^{+}+8 \mathrm{e}^{-} \rightarrow \mathrm{CH}_{4}+3 \mathrm{H}_{2} \mathrm{O}
$$

Combining equation 3 and 4, this leads to the overall methanogenesis reaction:

$$
\mathrm{CH}_{3} \mathrm{COO}^{-}+\mathrm{H}_{2} \mathrm{O} \rightarrow \mathrm{CH}_{4}+\mathrm{HCO}_{3}^{-}
$$

The Gibbs free energy of the overall methanogenesis reaction at standard conditions is $-76 \mathrm{~kJ} / \mathrm{mol}$ acetate, corresponding to $-9.5 \mathrm{~kJ} / \mathrm{mol} \mathrm{e}^{-}$.

For sulfate reduction, the reduction reaction is:

$$
\mathrm{SO}_{4}^{2-}+9 \mathrm{H}^{+}+8 \mathrm{e}^{-} \rightarrow \mathrm{HS}^{-}+4 \mathrm{H}_{2} \mathrm{O}
$$

Combining equation 6 with equation 3 leads to the overall sulfate reduction reaction:

$$
\mathrm{CH}_{3} \mathrm{COO}^{-}+\mathrm{SO}_{4}^{2-} \rightarrow \mathrm{HS}^{-}+2 \mathrm{HCO}_{3}^{-}
$$

The Gibbs free energy of the overall sulfate reduction reaction at standard conditions is $-48 \mathrm{~kJ} / \mathrm{mol}$ acetate, corresponding to $-6 \mathrm{~kJ} / \mathrm{mol} \mathrm{e}^{-}$. 

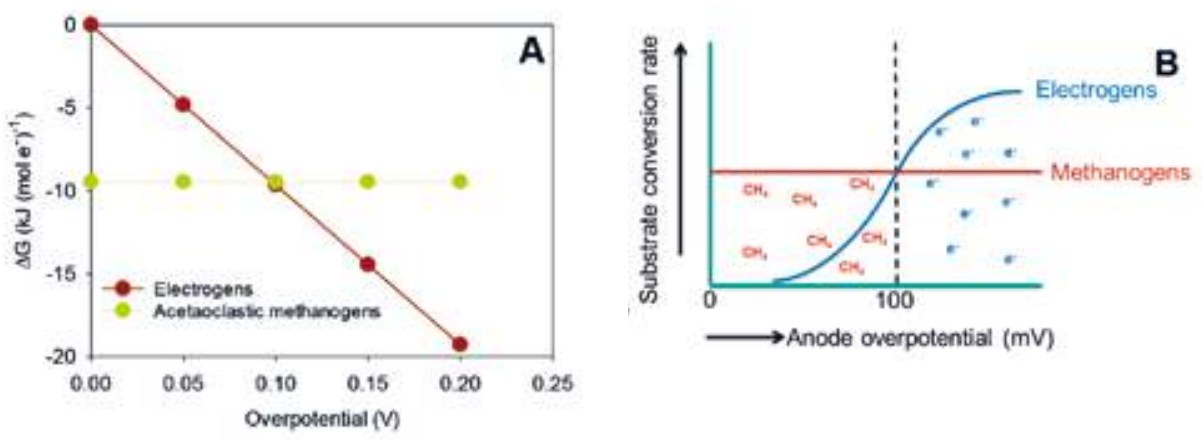

FIGURE 3 | The Gibbs free energy change for acetate oxidation by electrogens is a function of the anode potential, while the growth rate of methanogens using acetate as the electron donor is independent of the anode overpotential (left, figure A). For electrogens, at lower overpotential, catabolic reaction rates - and thus apparent maximum growth - follows Boltzmann statistics, while at higher overpotentials reaction rates and growth are limited by kinetic constants not related to anode potential. This way, an S-shaped curve is obtained relating apparent maximum growth rate of electrogens to anode potential, as is the case in the Butler-Volmer model. Methanogen growth kinetics are independent from anode potential. Provided higher attainable maximal growth rates for electrogens, at low anode overpotentials, growth of methanogens will dominate, while at higher overpotentials, electrogens will outgrow methanogens (right, figure B).

The Gibbs free energy for methanogenesis and sulfate reduction is not dependent on the potential of the anode, but only determined by the chemical species used in the reaction. Of course, this is under the assumption that both methanogens and sulfate reducers do not interact with the anode. For the bioanode, however, the Gibbs free energy for the oxidation reaction, that uses the anode as the electron acceptor, is directly dependent on the (electric) potential of the anode, which can be controlled at any value. The Gibbs free energy for a bioanode can thus be calculated as a function of the anode overpotential, which is defined as the difference between the theoretical potential of acetate oxidation and the electric potential of the anode. The result is shown in Figure 3A.

From Figure 3A it becomes clear that, under standard conditions, at an overpotential of $>100 \mathrm{mV}$, theoretically, the electrogens can gain more energy from the oxidation of acetate on a per mol basis than methanogens. Whether this may also lead to higher growth rates for electrogens than for methanogens, and thus to desired reaction specificity, can not be concluded from this strictly thermodynamic equilibrium approach. For this, reaction kinetics and growth parameters have to be taken into account. 


\subsection{REACTION KINETICS AND GROWTH PARAMETERS}

When considering competition between (functional) species for a single substrate, this is often done in terms of their apparent Monod-parameters: maximum growth rate $\left(\mu_{\max }\right)$ and substrate affinity (Ks). When these two apparent parameters are known and valid under the reactor conditions applied, they allow calculation of the specific growth rates for each species using the Monod equation. The specific growth rate is important because it relates to the substrate conversion rate and, thus, the current or methane production rate. The growth rate $\mu\left(\mathrm{d}^{-1}\right)$ is related to the substrate consumption rate $r_{s}\left(g\right.$ substrate-C $\left.\cdot L^{-1} \cdot d^{-1}\right)$ through:

$$
r_{s}=-\frac{\mu X}{Y}
$$

where $\mathrm{X}$ is the total biomass concentration $\left(\mathrm{g} \mathrm{L}^{-1}\right)$ and $\mathrm{Y}$ the yield coefficient of substrate conversion to biomass ( $\mathrm{g}$ biomass- $\mathrm{C} /(\mathrm{g}$ substrate- $\mathrm{C})$ ).

Where one set of apparent values for $\mu_{\max }, K_{s}$ and $Y$ may hold for methanogenesis at different anode potentials under further unchanging reactor conditions, this is not the case for electrogens: for these, anode potential may have profound effects on all three of them. As the anode overpotential defines the free energy change available to the electrogens, this may impact the yield coefficient. With more exergy per substrate molecule converted, less substrate conversion is required for obtaining a unit amount of biomass. That electrogenic bacteria are in fact able to exploit increased overpotentials for higher energy gain on a per substrate molecule basis is illustrated by reported yield values, which are, depending on applied anode potential, ranging from 0 to $0.54 \mathrm{~g}$ biomass-C per g substrate-C used when acetate was used as the substrate ${ }^{[13,22,23]}$.

Moreover, at lower overpotentials, reaction rates for electrogens may be severely limited by kinetics more accurately described by a form of Transition State Theory (TST), a good example of which for electrode-redox reactions is the Butler-Volmer equation. Incorporating a form of TST into the electrogenic growth model, may allow for adequate description of the substantially higher apparent maximal growth rates and lower Ks values observed when more overpotential is applied ${ }^{[35]}$.

The models for electrogenic growth resulting when TST elements are combined with the law of mass action principle of Monod, show a typical S-shaped response of (apparent maximum) growth rates on anode overpotential (Figure 3B) ${ }^{[2,35]}$. Where, for methanogens, substrate affinity has been analyzed thoroughly and ranges from relatively low values of $0.5 \mathrm{mM}$ for Methanotrix to relatively high values of $3.0 \mathrm{mM}$ for Methanosarcina sp. ${ }^{[25]}$, up to date only a few studies have been performed that aimed at determining apparent growth parameters of electrogens 
as function of overpotential. For example, Lee et al. ${ }^{[24]}$ report only a small variation of $K_{\mathrm{s}}$ as a function of anode overpotential, while Hamelers et al. ${ }^{[26]}$ report two different $K_{\mathrm{s}}$ values for two different overpotentials. They found a $K_{\mathrm{s}}$ value of 0.35 $\mathrm{mM}$ at $0.1 \mathrm{~V}$ overpotential and of $2.2 \mathrm{mM}$ at $0.2 \mathrm{~V}$ overpotential. This would mean that, in contrast to what was discussed before, at low overpotential, electrogens have a lower affinity constant and, assuming unaltered growth yield, a competitive advantage over methanogens. In practice, however, bioanodes show higher values for $\mathrm{CE}$ at higher overpotentials and, therefore, do have a competitive advantage over methanogens. The analyzed values were determined without taking biofilm growth into account and the effect of overpotential on the growth yield ${ }^{[27]}$.

Clearly, there is a need for more detailed studies to determine the growth constants for mixed cultures in bioanodes and to relate these growth constants to overpotential to gain more insight into using substrate concentration and overpotential in a combined control strategy to prevent methanogenesis. When revised models would be accurately fitted on experimental data, this would allow for more sophisticated analysis of methanogenic/electrogenic growth while competing for substrate, and identification of "sweet" spots where reaction specificity towards current production is optimal.

\subsection{HIGH VOLUMETRIC ANODE SURFACE AREA IS KEY TO ACHIEVE HIGH RATES AT LOW SUBSTRATE CONCENTRATIONS}

As was explained in more detail in the previous section, electrogens and methanogens are competing for substrate in MFCs, and this competition can be influenced by adjusting anode potential. Therefore, achieving acetate concentrations as low as possible while minimizing the required costs in form of additional overpotential will be key in obtaining high MFC performance. Herein, a sensitive aspect may be formed by mass transfer limitations. Figure 4A shows an example of how acetate concentration affects the current produced by a bioanode, at different anode potentials. These experiments were performed to analyze bioanode performance with impedance spectroscopy, and the details of experimental methods can be found inter Heijne et $\mathrm{al}^{[28]}$. At low acetate concentrations, beneath $1 \mathrm{mM}$, produced currents are to a large extent mass transfer limited and less dependent on applied overpotential. However, once the (bulk) acetate concentrations are higher than $1 \mathrm{mM}$, the higher overpotentials produce most current, indicating electrode potential to be the limiting factor in current production.

Apart from stimulating process selectivity, controlling the substrate concentrations at low levels is an attractive operational strategy as when considering 

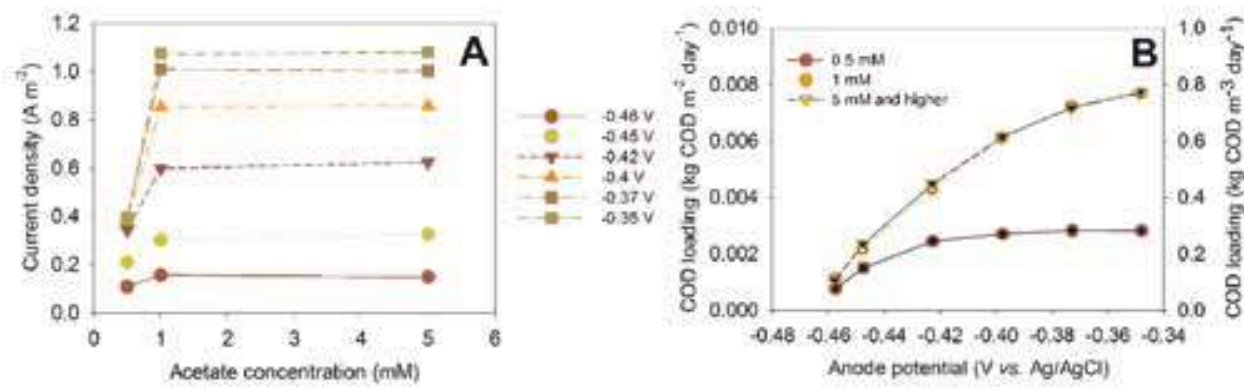

FIGURE 4 | (A) Current density as a function of acetate concentration at different overpotentials (calculated from reference 28) and (B) the same current density represented as COD loading as a function of the anode potential. The COD loading is expressed per $\mathrm{m}^{2}$ of anode surface area (left axis) and per $\mathrm{m}^{3}$ of reactor using a specific anode surface area of $100 \mathrm{~m}^{2} \cdot \mathrm{m}^{-3}$.

wastewater treatment, it is crucial to remove as much waste from the wastewater as possible; meaning that the outflow concentration of a biodegradable material (and thus, concentration inside the reactor) needs to be low. It is therefore inherent for application in wastewater treatment to operate the system at low substrate concentrations.

To achieve a low substrate concentration, while minimizing mass transfer losses, the specific electrode surface area can be adjusted. High specific surface area is required, because when operating at lower substrate concentration, also the current is low, considering that (very) high overpotentials are undesirable. Figure 4B presents the data from Figure 4A in an alternative way. It shows the COD loading rate required to maintain a certain substrate concentration $(0.5,1$ or $>5 \mathrm{mM}$ ) as a function of anode overpotential. COD loading rate is expressed both per anode surface $\left(\mathrm{m}^{2}\right)$ and per reactor volume, assuming a specific surface area of $100 \mathrm{~m}^{2} \cdot \mathrm{m}^{-3}$. This picture shows that at overpotentials that are competitive with methanogens on a free energy basis $(<100 \mathrm{mV})$, still over $0.6 \mathrm{~kg}$ of COD can be removed per $\mathrm{m}^{3}$ of reactor per day. When a higher specific surface area would be applied in the same volume, even higher loading could be applied in the same volume, leading to higher volumetric conversion rates.

To increase this specific surface area, high surface area (porous) materials, like carbon granules, graphite brushes and graphite felt ${ }^{29-31}$, are used. A problem often encountered with high specific surface materials is that they are often used in systems with a considerable distance between the anode and cathode, while the electrolyte has a typical limited conductivity (in the range of 1 to $5 \mathrm{~ms} \cdot \mathrm{cm}^{-1}$ ). For every electron that is generated, charge needs to be transported via an ionic current from the place where the electron is generated to the place where it is reduced at the cathode. A low conductivity wastewater limits the amount of ions present that can take part in this charge transport through the electrolyte and, 
therefore, increases the ionic resistance. For example, the ohmic voltage drop over $1 \mathrm{~cm}$ of electrolyte is already around $0.5 \mathrm{~V}$ at $10 \mathrm{Am}^{-2}$ for a typical waste stream with a conductivity of $2 \mathrm{mS} \cdot \mathrm{cm}^{-1}{ }^{[2]}$. Another risk with porous electrode materials is the occurrence of increased concentration polarization, as electrolyte mixing by convection is limited in the confined space of the pores.

An example of a strategy to combine a high specific surface area and ionic charge transfer has been proposed in the form of a fluidized capacitive MFC ${ }^{[32-34]}$. In this fluidized reactor design, the anode consists of activated carbon granules that are charged and discharged in separate places. During conversion of acetate, electrons are stored in the electric double layer on the surface of the activated carbon granules. Discharge can occur in a well-designed discharge cell at locally high conductivity, thereby reducing the ohmic losses in the discharge part of the system. Further study into scalable reactor designs, in which the limitations in current by a (locally) low substrate concentration can be overcome, is important to bring bioanodes and BESs relying on bioanodes further towards application.

\subsection{CONCLUSIONS}

Many researchers in the field of BESs do not see a future in MFCs for the treatment of wastewater and the recovery of energy. The strategies proposed in this paper are essential for MFCs to live up to their promise and become practically applicable as a mature wastewater treatment technology. We have argued that low substrate concentration and optimized anode potential are both favorable to achieve a high CE. For MFCs to become a competitive technology in wastewater treatment, this high $\mathrm{CE}$ is crucial. At the same time, low substrate concentrations (effluent concentrations) need to be combined with high volumetric substrate loading rates and current density; meaning that high specific surface areas are needed.

\subsection{ACKNOWLEDGEMENTS}

This work was performed in the cooperation framework of Wetsus, European center of excellence for sustainable water technology (www.wetsus.nl). Wetsus is co-funded by the Dutch Ministry of Economic Affairs and the Ministry of Infrastructure and Environment, the European Union Regional Development Fund, the Province of Fryslân and the Northern Netherlands Provinces. The authors would like to thank the participants of the research theme "Resource Recovery” for the fruitful discussions and their financial support. 


\subsection{REFERENCES}

1. Logan, B.E.; Hamelers, B.; Rozendal, R.; Schröder, U.; Keller, J.; Freguia, S.; Aelterman, P.; Verstraete, W.; Rabaey, K. Microbial fuel cells: Methodology and technology. Environ. Sci. Technol. 2006, 40, 5181-5192.

2. Rozendal, R.A.; Hamelers, H.V.M.; Rabaey, K.; Keller, J.; Buisman, C.J.N. Towards practical implementation of bioelectrochemical wastewater treatment. Trends Biotechnol. 2008, 26, 450-459.

3. Logan, B.E.; Call, D.; Cheng, S.; Hamelers, H.V.M.; Sleutels, T.H.J.; Tom, H.J.A.; Jeremiasse, A.W.; Rozendal, R.A. Microbial Electrolysis Cells for High Yield Hydrogen Gas Production from Organic Matter. Environ. Sci. Technol. 2008, 42, 8630-8640.

4. Logan, B.E.; Rabaey, K. Conversion of wastes into bioelectricity and chemicals by using microbial electrochemical technologies. Science 2012, 337, 686-690.

5. Rodríguez Arredondo, M.; Kuntke, P.; Jeremiasse, A.W.; Sleutels, T.H.J.A.; Buisman, C.J.N.; ter Heijne, A. Bioelectrochemical systems for nitrogen removal and recovery from wastewater. Environ. Sci. Water Res. Technol. 2015, 1, 22-33.

6. Kuntke, P.; Sleutels, T.H.J.A.; Saakes, M.; Buisman, C.J.N. Hydrogen production and ammonium recovery from urine by a Microbial Electrolysis Cell. Int. J. Hydrog. Energy 2014, 39, 4771-4778.

7. Ledezma, P.; Kuntke, P.; Buisman, C.J.N.; Keller, J.; Freguia, S. Source-separated urine opens golden opportunities for microbial electrochemical technologies. Trends Biotechnol. 2015, 33, 214-220.

8. Inglesby, A.E.; Fisher, A.C. Enhanced methane yields from anaerobic digestion of Arthrospira maxima biomass in an advanced flow-through reactor with an integrated recirculation loop microbial fuel cell. Energy Environ. Sci. 2012, 5, 7996-8006.

9. De Vrieze, J.; Gildemyn, S.; Arends, J.B.A.; Vanwonterghem, I.; Verbeken, K.; Boon, N.; Verstraete, W.; Tyson, G.W.; Hennebel, T.; Rabaey, K. Biomass retention on electrodes rather than electrical current enhances stability in anaerobic digestion. Water Res. 2014, 54, 211-221.

10. Rabaey, K.; Bützer, S.; Brown, S.; Keller, J.; Rozendal, R.A. High current generation coupled to caustic production using a lamellar bioelectrochemical system. Environ. Sci. Technol. 2010, 44, 4315-4321.

11. Rozendal, R.A.; Leone, E.; Keller, J.; Rabaey, K. Efficient hydrogen peroxide generation from organic matter in a bioelectrochemical system. Electrochem. Commun. 2009, 11, 1752-1755.

12. Hamelers, H.V.M.; Sleutels, T.H.J.A.; Jeremiasse, A.W.; Post, J.W.; Strik, D.P.B.T.B.; Rozendal, R.A. Technological Factors Affecting BES Performance and Bottlenecks Towards Scale Up. In Bio-Electrochemical Systems: From Extracellular Electron Transfer to Biotechnological Application; Rabaey, K., Angenent, L.T., Schroder, U., Keller, J., Eds.; IWA Publishing: London, UK, 2009.

13. Aelterman, P.; Freguia, S.; Keller, J.; Verstraete, W.; Rabaey, K. The anode potential regulates bacterial activity in microbial fuel cells. Appl. Microbiol. Biotechnol. 2008, 78, 409-418.

14. Tchobanoglous, G.; Burton, F.L.; Stensel, H.D. Wastewater Engineering: Treatment and Reuse, 3rd ed.; Metcalf, L., Eddy, H.P., Eds.; McGraw-Hill: New York, NY, USA, 2003.

15. Agler, M.T.; Wrenn, B.A.; Zinder, S.H.; Angenent, L.T. Waste to bioproduct conversion with undefined mixed cultures: The carboxylate platform. Trends Biotechnol. 2011, 29, 70-78.

16. Kadier, A.; Simayi, Y.; Kalil, M.S.; Abdeshahian, P.; Hamid, A.A. A review of the substrates used in microbial electrolysis cells (MECs) for producing sustainable and clean hydrogen gas. Renew. Energy 2014, 71, 466-472. 
17. Pant, D.; van Bogaert, G.; Diels, L.; Vanbroekhoven, K. A review of the substrates used in microbial fuel cells (MFCs) for sustainable energy production. Bioresour. Technol. 2010, 101, 1533-1543.

18. Visser, A. The Anaerobic Treatment of Sulfate Containing Wastewater. Ph.D. Thesis, Wageningen University, 1995.

19. Sleutels, T.H.J.A.; Hamelers, H.V.M.; Rozendal, R.A.; Buisman, C.J.N. Ion transport resistance in Microbial Electrolysis Cells with anion and cation exchange membranes. Int. J. Hydrog. Energy 2009, 34, 3612-3620.

20. Sleutels, T.H.J.A; Darus, L.; Hamelers, H.V.M.; Buisman, C.J.N. Effect of operational parameters on Coulombic efficiency in bioelectrochemical systems. Bioresour. Technol. 2011, 102, 11172-11176.

21. Heijnen, S.J. Thermodynamics of Microbial-Growth And Its Implications For Process Design. Trends Biotechnol. 1994, 12, 483-492.

22. Freguia, S.; Rabaey, K.; Yuan, Z.G.; Keller, J. Electron and carbon balances in microbial fuel cells reveal temporary bacterial storage behavior during electricity generation. Environ. Sci. Technol. 2007, 41, 2915-2921.

23. Picioreanu, C.; Head, I.M.; Katuri, K.P.; van Loosdrecht, M.C.M.; Scott, K. A computational model for biofilm-based microbial fuel cells. Water Res. 2007, 41, 2921-2940.

24. Lee, H.-S.; Torres, C.I.; Rittmann, B.E. Effects of Substrate Diffusion and Anode Potential on Kinetic Parameters for Anode-Respiring Bacteria. Environ. Sci. Technol. 2009, 43, 7571-7577.

25. Jetten, M.S.M.; Stams, A.J.M.; Zehnder, A.J.B. Methanogenesis from acetate: A comparison of the acetate metabolism in Methanothrix soehngenii and Methanosarcina spp. FEMS Microbiol. Lett. 1992, 88, 181-198.

26. Hamelers, H.V.M.; ter Heijne, A.; Stein, N.; Rozendal, R.A.; Buisman, C.J.N. Butler-Volmer-Monod model for describing bio-anode polarization curves. Bioresour. Technol. 2011, 102, 381-387.

27. Rodriguez, J.; Lema, J.M.; Kleerebezem, R. Energy-based models for environmental biotechnology. Trends Biotechnol. 2008, 26, 366-374.

28. Ter Heijne, A.; Schaetzle, O.; Gimenez, S.; Navarro, L.; Hamelers, B.; Fabregat-Santiago, F. Analysis of bio-anode performance through electrochemical impedance spectroscopy. Bioelectrochemistry 2015 , $106,64-72$.

29. Rabaey, K.; Clauwaert, P.; Aelterman, P.; Verstraete, W. Tubular Microbial Fuel Cells for Efficient Electricity Generation. Environ. Sci. Technol. 2005, 39, 8077-8082.

30. Sleutels, T.H.J.A.; Lodder, R.; Hamelers, H.V.M.; Buisman, C.J.N. Improved performance of porous bio-anodes in microbial electrolysis cells by enhancing mass and charge transport. Int. J. Hydrog. Energy 2009, 34, 9655-9661.

31. Logan, B.; Cheng, S.; Watson, V.; Estadt, G. Graphite fiber brush anodes for increased power production in air-cathode microbial fuel cells. Environ. Sci. Technol. 2007, 41, 3341-3346.

32. Deeke, A.; Sleutels, T.H.J.A.; Donkers, T.F.W.; Hamelers, H.V.M.; Buisman, C.J.N.; Ter Heijne, A. Fluidized Capacitive Bioanode As a Novel Reactor Concept for the Microbial Fuel Cell. Environ. Sci. Technol. 2014, 49, 1929-1935.

33. Li, J.; Ge, Z.; He, Z. A fluidized bed membrane bioelectrochemical reactor for energy-efficient wastewater treatment. Bioresour. Technol. 2014, 167, 310-315.

34. Liu, J.; Zhang, F.; He, W.; Zhang, X.; Feng, Y.; Logan, B.E. Intermittent contact of fluidized anode particles containing exoelectrogenic biofilms for continuous power generation in microbial fuel cells. J. Power Sour. 2014, 261, 278-284.

35. Desmond-Le Quéméner, E.; Bouchez, T. A thermodynamic theory of microbial growth. The ISME Journal. 2014, 8, 1747-1751 


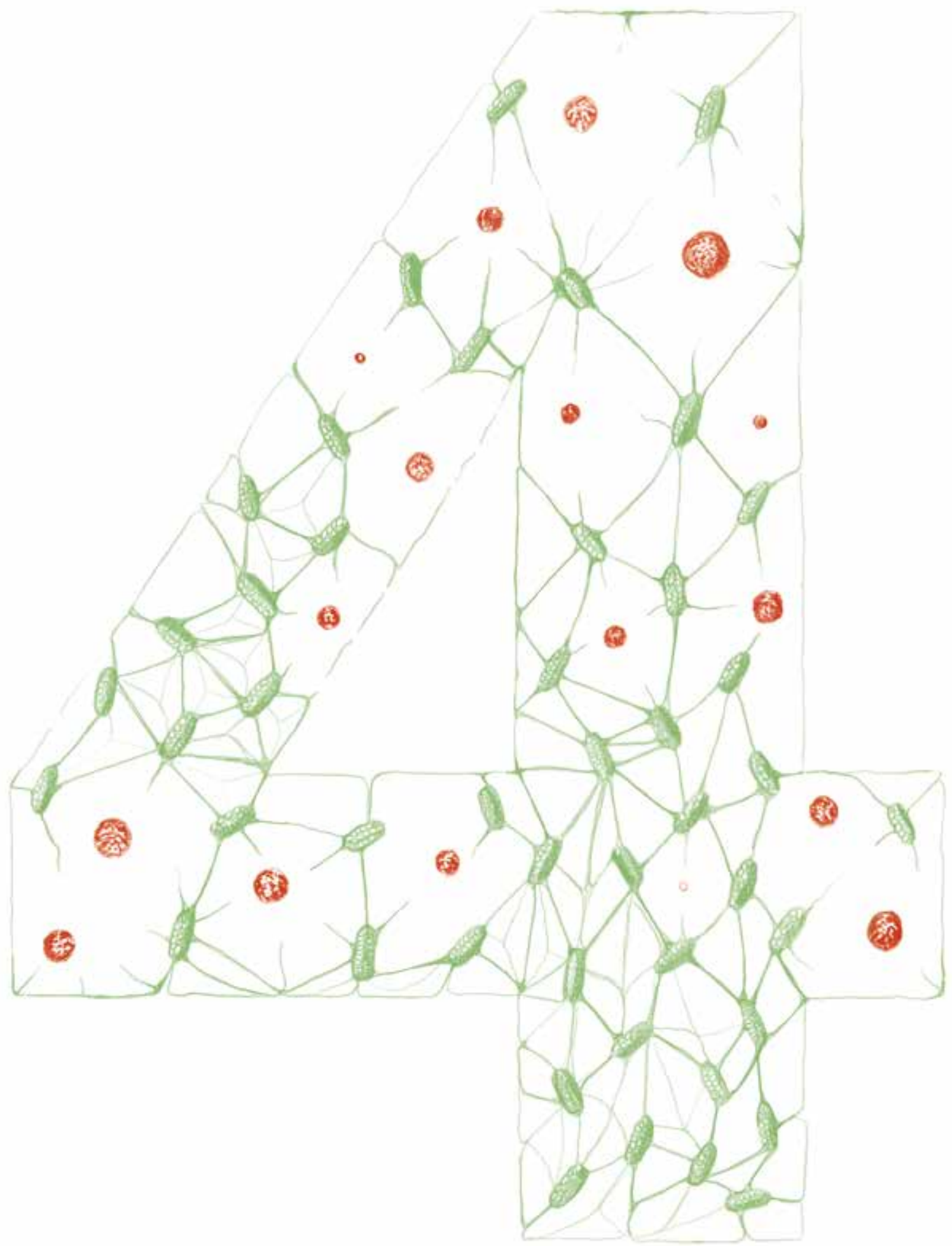




\section{COMPETITION BETWEEN METHANOGENS AND ACETOGENS IN BIOCATHODES: A COMPARISON BETWEEN POTENTIOSTATIC AND GALVANOSTATIC CONTROL}

Being essential to the charging process of a Microbial Rechargeable Battery, Microbial electrosynthesis is a useful technology for the renewable production of organic commodities from biologically catalyzed reduction of $\mathrm{CO}_{2}$. However, for the technology to become applicable, process selectivity, stability and efficiency needs further improvements. In this chapter, the effect of different electrochemical control modes (potentiostatic/galvanostatic) on both the start-up characteristics and steady-state performance of biocathodes using a non-enriched mixed culture inoculum is reported. Based on presented results, it is concluded that kinetic differences exist between the two dominant functional microbial groups (i.e. homoacetogens and methanogens) and that by applying different current densities, these differences can be exploited to steer product selectivity and reactor performance. Therefore, through future optimizations by these means, acetate producing MES may be sustained at high production rates and product selectivity without the need of methanogen-inhibiting chemical additives or pre-enrichment of inoculum.

This chapter has been published as: Molenaar, S.D.; Saha, P.; Mol, A.R.; Sleutels, H.J.A.; ter Heijne, A.; Buisman, C.J.N., Competition between methanogens and acetogens in biocathodes: a comparison between potentiostatic and galvanostatic control, International Journal of Molecular Sciences, 2017, 18, 204, doi:10.3390/ijms18010204 


\subsection{INTRODUCTION}

The use of bioelectrochemical systems (BES) for the production of organic compounds such as alcohols or short to medium chain fatty acids through microbial electrosynthesis (MES) holds potential ${ }^{[1]}$. By exploiting the capabilities of microorganisms to metabolize using electrodes as electron donor - a feature that is found to be widespread amongst most diverse groups of microorganisms ${ }^{[2]}$ - a variety of organic compounds can be synthesized, at mild operation conditions ${ }^{[3-5]}$. As such, these systems provide an environmentally friendly means to produce useful chemicals using cheap and available substrates (carbon dioxide and water), while avoiding the requirement of scarce metal catalysts in the cathode, as would be the case in most conventional electrosynthetic processes.

While the products potentially formed in MES are found to be diverse, main scientific efforts in this field have so far focused on the specific production of acetate by the reduction of carbon dioxide ${ }^{[6-11]}$. There are two general considerations at the basis of this product choice: (1) specificity and (2) reaction rate. With the standard reduction potential of acetate from $\mathrm{CO}_{2}$ being highest amongst all carboxylates (and considerably higher than that of formate), the potential window within which cathodic production of acetate can take place shows minimal overlap with respect to most alternative $\mathrm{CO}_{2}$ reduction products. This provides a thermodynamic advantage regarding the specificity towards production of acetate. In addition, with the number of electrons (8) still being considerably low compared to higher reduction products like butyrate, reaction rates are not severely kinetically limited, and production of acetate at high specificity, up to $100 \%$, and in some cases at high rates, up to $66 \mathrm{~kg} \mathrm{~m}^{-3} \mathrm{day}^{-1}$, has been achieved in biocathodes ${ }^{[7]}$.

Troubling to the specific and efficient production of acetate in BES is the production of methane by reduction of carbon dioxide, with a very comparable standard reduction potential (Table 1).

As follows from Table 1, given its standard potential, methanogenesis may always be regarded competitive to acetate production from a thermodynamic perspective, with methane either produced from $\mathrm{H}_{2}$ and $\mathrm{CO}_{2}$ instead of acetate (in case higher overpotentials are used), or produced acetate being consumed through the disproportionation reaction used in acetoclastic methanogenesis.

Although the production of methane at biocathodes may form a target in itself, with applications in for instance biogas upgrading or the conversion of organic waste into natural gas ${ }^{[13,14]}$, the distinct uses and higher economical value of acetate make taking control over specificity in biocathodes regarding production of both compounds worthwhile. Substantial scientific effort to this end has been made, using diverse methodologies ${ }^{[3,8,10,15-17]}$. The findings are summarized in Figure 1, which depicts the possible mechanisms for production of both acetate and methane in MES, including references proposing these mechanisms. 
TABLE 1 | Overview of reactions involved in methanogenesis and homoacetogenesis by reduction of $\mathrm{CO}_{2}$. Depicted are both half reactions (upper) and overall reactions using hydrogen or acetate as electron donor. Standard reaction energies / potentials were taken from Thauer et al. ${ }^{[12]}$ and expressed at pH 7, $298 \mathrm{~K}$ with all other reactants at standard concentrations.

\begin{tabular}{|l|l|l|l|}
\hline Process & Reaction & \multicolumn{2}{|l|}{$\mathrm{E}_{0}^{\prime} / \mathrm{V}$ (vs. Ag/AgCl) } \\
\hline Half reactions & & & \\
Methanogenesis & $\mathrm{CO}_{2}+8 \mathrm{e}^{-}+8 \mathrm{H}^{+} \Leftrightarrow \mathrm{CH}_{4}+2 \mathrm{H}_{2} \mathrm{O}$ & -0.24 & \\
Acetogenesis & $2 \mathrm{CO}_{2}+8 \mathrm{e}^{-}+8 \mathrm{H}^{+} \Leftrightarrow \mathrm{CH}_{3} \mathrm{COOH}+2 \mathrm{H}_{2} \mathrm{O}$ & -0.29 & \\
Hydrogen oxidation & $2 \mathrm{H}^{+}+2 \mathrm{e}^{-} \Leftrightarrow \mathrm{H}_{2}$ & -0.41 & \\
Overall reactions in anaerobic digestion & & & $\Delta \mathrm{G}_{\mathrm{r}}\left(\mathrm{kJ} \cdot \mathrm{mol}^{-1}\right)$ \\
Homoacetogenesis & & 0.12 & -95 \\
Hydrogenotrophic methanogenesis & $4 \mathrm{H}_{2}+\mathrm{CO}_{2} \Leftrightarrow \mathrm{CH}_{4}+2 \mathrm{H}_{2} \mathrm{O}$ & 0.17 & -131 \\
Acetoclastic methanogenesis & $\mathrm{CH}_{3} \mathrm{COOH} \Leftrightarrow \mathrm{CH}_{4}+\mathrm{CO}_{2}$ & 0.05 & -36 \\
\hline
\end{tabular}

For the production of acetate, two strategies to prevent methanogenesis may be distinguished: (1) pre-enrichment of inoculum or the use of pure cultures, while working with aseptic conditions to prevent contamination of the systems with methanogens, thus effectively keeping out competitive microorganisms ${ }^{[17]}$ and (2) chemical inhibition of methanogenesis ${ }^{[7]}$. Although these techniques have shown successful to some extent, none of these practices alone provide a means for continuous, larger scale and long term septic reactor operation. The use of pure cultures or enriched inocula is prone to contamination and use of chemical inhibitors is unattractive both from economical as well as environmental perspective. Additionally, methanogens may develop immunity towards the inhibiting compounds used, rendering them less effective over time ${ }^{[19]}$.

The need thus exists for a more integrated approach towards the selective and stable production of acetate, which takes into account (1) structural differences between microbial groups, (2) differences in thermodynamics, and (3) kinetics associated with the proposed pathways in Figure 1 to affect product specificity. Based on these aspects, reactor operational parameters need to be found at which biomass retention time for acetogens is higher than for methanogens, and/or keeping growth rates (and thus metabolic activity) of acetogens higher than those of methanogens.

Tangent to the considerations of fast kinetics and long acetogenic biomass retention is the question whether biocathodes are best operated using potentiostatic or galvanostatic control: A constant potential may be set to narrow down the possibly involved electron transfer mechanisms (Figure 1) and may lead to the formation of 


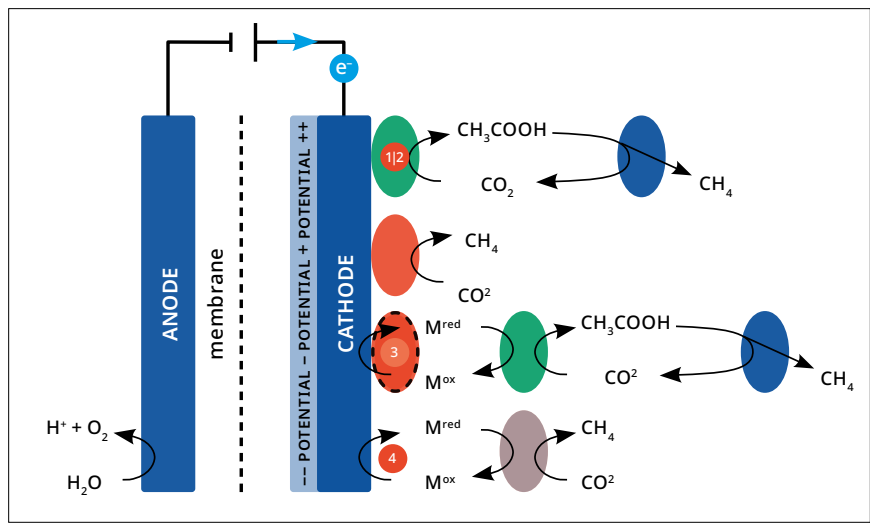

FIGURE 1 | Different metabolic pathways in a mixed culture biocathode as proposed in different studies. Distinction can be made between direct electron transfer ${ }^{[18]}$, in which microbes are directly attached to the electrode surface, thus allowing electrons to reach the intracellular environment by means of electrical conductance, and the use of a redox active chemical mediator $\left(M_{o x} / M_{\text {red }}\right)$ in the case of indirect electron transfer. Several studies have suggested a combination, in which direct electron transfer leads to the biocatalyzed reduction of a mediator compound, which then functions as electron donor for suspended cultures $^{[7,8]}$. Regardless whether initial electron transfer takes place directly or indirectly, a close competition between methanogens (brown) and acetogens (green) will exist for available electrons. Regarding the composition of a possible mediator, although in theory any kind of mediator could be shuttling electrons back and forth between electrode and microbe as long as its reversible redox potential is adequate, in practice this role is most likely fulfilled by hydrogen, although formate has been hypothesized to play a role as secondary mediator as well ${ }^{[1]}$. Any formed acetate can be further oxidized acetoclastically to form $\mathrm{CO}_{2}$ and methane (blue microbe). The red-circled numbers refer to the potential levels tested in this study, aimed at establishing indicated pathways and are further defined in the materials and methods section.

a more energy efficient microbial community. Constant current, on the other hand, allows for better control over production rates and possibly selectivity as it is less sensitive or dependent on (local) changes in e.g. pH, and the electron flux can be controlled to match stoichiometrically with the rate of $\mathrm{CO}_{2}$ supply.

In this study, the performance of graphite felt electrodes was tested for the cathodic production of acetate using a mixed culture inoculum from AD sludge, using continuously operated reactors. The effect of using both potentiostatic and galvanostatic control on reactor performance was analyzed and compared. By using a mixed culture inoculum, presence of both acetogens and methanogens was assured. Different cathode potentials were tested during potentiostatic experiments, and acetate production was compared to current controlled experiments, in which reducing equivalents were provided at constant rate. Results were interpreted to provide more insight in the mechanisms at play in biocathodes, and the consequential perspectives on future reactor optimization. 


\subsection{MATERIALS AND METHODS}

\subsubsection{System design and reactor assembly}

Experiments were conducted in electrochemical cells consisting of two plexiglass flow compartments, two flat plate electrodes and two plexiglass support plates as described previously ${ }^{[3]}$.The assembly had an effective projected electrode surface area of $22 \mathrm{~cm}^{2}$ for both cathode and anode and an effective cathode volume of $33 \mathrm{~cm}^{3}$. Cathodes consisted of a stainless steel (SS316) plate serving as current collector, with a plain sheet of graphite paper and 5 layers of graphite felt (thickness $3 \mathrm{~mm}$, FMI Composites Ltd., Scotland) firmly held between current collector and membrane, thus with the felt serving as the actual electrode material in contact with the electrolyte. Anodes consisted of flat platinum/iridium coated titanium plates ( $\mathrm{Pt} / \mathrm{IrO}_{2}$ 80:20, Magneto special anodes BV, Schiedam, the Netherlands) with the anode flow compartment filled with polypropylene spacer material sandwiched between the anode and membrane, ensuring proper flow distribution and providing support to the membrane. An $\mathrm{Ag} / \mathrm{AgCl}$ reference electrode (model QM711X/Gel, Prosense, Oosterhout, Netherlands) was connected to the cathode using a Haber-Lugging capillary filled with saturated $3 \mathrm{M} \mathrm{KCl} \mathrm{(+0.205} \mathrm{V} \mathrm{vs.} \mathrm{NHE).}$ All potentials reported were measured relative to this reference. The anode and the cathode compartments were separated by a cation exchange membrane (Ralex ${ }^{\mathrm{TM}}$ C(M)H-PES, Vysočany, Czech Republic). Total catholyte recirculation volume was $180 \mathrm{~mL}$, anolyte recirculation volume $120 \mathrm{~mL}$. Electrolytes were recirculated at a pump speed of $80 \mathrm{~mL} \cdot \mathrm{min}^{-1}$. In-line $\mathrm{pH} /$ temperature monitoring was performed on the catholyte (Endress+Hauser, sensor model CP571D-7BV21 connected to a Liquiline datalogger). Temperature of reactors was maintained constant at $32 \pm 1$ ${ }^{\circ} \mathrm{C}$ using climate control of the research cabinet, and systems were shielded from light to avoid the possibility of photoautotrophic activities during the experiments. An Ivium N-stat multichannel DC potentiostat was used in a three-electrode configuration (working, counter, and reference) to perform electrochemical measurements and experiments. Recording and analysis of potentiostat data was carried out using the Iviumstat software.

\subsubsection{Electrolyte composition}

Catholyte was made using MilliQ water containing $0.4 \mathrm{~g} \cdot \mathrm{L}^{-1} \mathrm{NH}_{4} \mathrm{HCO}_{3}, 0.05 \mathrm{~g} \cdot \mathrm{L}^{-1}$ $\mathrm{Ca}(\mathrm{OH})_{2}, 0.1 \mathrm{~g} \cdot \mathrm{L}^{-1} \mathrm{MgSO}_{4} \cdot 7 \mathrm{H}_{2} \mathrm{O}, 0.87 \mathrm{~g} \cdot \mathrm{L}^{-1} \mathrm{~K}_{2} \mathrm{HPO}_{4}, 0.68 \mathrm{~g} \cdot \mathrm{L}^{-1} \mathrm{KH}_{2} \mathrm{PO}_{4}, 1 \mathrm{~mL} \cdot \mathrm{L}^{-1}$ of vitamin solution ${ }^{[3]}$ and $1 \mathrm{~mL} \cdot \mathrm{L}^{-1}$ of trace metals solution ${ }^{[34]}$. In order to ensure anaerobic conditions and high concentrations of $\mathrm{CO}_{2}$ in the influent, the medium was continuously purged with $\mathrm{CO}_{2}$ prior to injection. Using this practice, a total 
inorganic carbon concentration (TIC) of $11 \mathrm{mM}$ was dissolved in the catholyte, buffering the $\mathrm{pH}$ of the catholyte at 5.5.

In later current controlled experiments, the methanogen inhibitor 2-bromoethanosulfonate (2-BES) was added in order to suppress methanogenic activities at a concentration of $2.1 \mathrm{~g} \cdot \mathrm{L}^{-1}$. Anolyte consisted of a $10 \mathrm{mM}$ phosphate buffer adjusted to $\mathrm{pH} 7$ before use. Anolytes were sparged continuously with $\mathrm{CO}_{2}$ to remove any formed oxygen throughout the experiments while preventing crossmembrane $\mathrm{CO}_{2}$ stripping.

\subsubsection{Inoculum}

A mixture of activated sludge from an anaerobic digester and cow manure was used as source of microorganisms. Homogenized activated sludge and cow manure were centrifuged separately for 5 minutes at $3700 \mathrm{rpm}$ and supernatant from both solutions were mixed in a 2:1 ratio, resulting in the final inoculum with a COD of $12.5 \mathrm{~g} \cdot \mathrm{L}^{-1}$. Upon inoculation, each cell was injected with $10 \mathrm{~mL}$ of inoculum leading to an initial COD of $0.7 \mathrm{~g} \cdot \mathrm{L}^{-1}$ in the catholyte at the start of biotic experiments.

\subsubsection{Hydraulic and electrochemical operational conditions}

Systems were filled and continuously fed with fresh electrolyte giving an HRT of 40 hours (potentiostatic experiments) or 20 hours (current controlled experiments). Prior to inoculation, potentials/currents were applied for at least 2 days until potentials/ currents stabilized, thus serving as baseline. In the first run, four cells were poised each at a different potential. Based on the equilibrium potentials for formation of hydrogen and acetate from $\mathrm{CO}_{2}$, the following four potentials were chosen:

- $\quad-560 \mathrm{mV}$; the standard equilibrium potential of direct carbon dioxide reduction into acetate is $-490 \mathrm{mV}$. The first setup aimed at facilitating this reaction by imposing only a small overpotential of $70 \mathrm{mV}$. Doing so, the formation of hydrogen gas is thermodynamically unfeasible and alternative electron transfer is therefore required. This may imply direct electron transfer, providing no other electron mediators are present in the system.

- $\quad-630 \mathrm{mV}$; the second potential was selected based on the same rationale as for setup 1 , but with a slightly increased overpotential.

- $\quad-700 \mathrm{mV}$; at this potential, hydrogen formation in theory becomes feasible but due to large overpotentials associated with hydrogen formation a catalyst would be required.

- $\quad-900 \mathrm{mV}$; well below the equilibrium potential for hydrogen formation in these systems, a small cathodic current of 2-4 mA was observed during the abiotic operation of the system, providing sufficient reductants at start. 
During the potential controlled experiments, systems were put in batch mode by stopping influent pumps directly after inoculation for 72 hours to prevent washout. After this initial batch phase, influents were resumed at the set HRT of 40 hours.

During the current controlled experiments all cells were set to an HRT of 20 hours, thereby allowing a fixed and equal flux of $\mathrm{CO}_{2}$ to all cathodes. HRT was maintained also directly after inoculation, as preliminary results showed good retention of biomass in the graphite felt even after prolonged periods of inactivity and continuous flushing.

\subsubsection{Analytical techniques}

Liquid and head space samples (1 mL) were taken from catholytes, once a week or more frequently when observed changes in currents/potentials or gas fluxes justified this. Occasionally, the anolyte was sampled for its ionic composition to assess crossover rates of ions. TIC/TOC measurements were done by using a Shimadzu TIC/TOC analyser; model TOC-VCPH in combination with Shimadzu ASI-V Autosampler. Gas samples were taken from the headspace in the cathode chamber and analysed for $\mathrm{H}_{2}, \mathrm{CO}_{2}, \mathrm{CH}_{4}$ and $\mathrm{O}_{2}$ using a gas chromatograph (Varian CP-4900 microGC, TCD detector, Mol Sieve 5A PLOT and PoraPlot U columns in parallel). Catholyte COD was determined using a spectrophotometer HACH XION 500 (HACH, Loveland, Colorado, USA) with test kit (No. 414, Hach-Lange, Tiel, The Netherlands). For obtaining estimates on biomass concentrations, COD of both unfiltered and filtered $(0.45 \mu \mathrm{m})$ electrolytes were compared. HPLC analyses for detection of organic acids (formic acid, acetic acid, propionic acid and butyric acid) were performed with a Dionex UHPLC system.

\subsubsection{Performance calculations}

With acetate being the target product, CE expresses the fraction of the electron flow (which is dictated by the current) which is used to form acetate:

$$
C E=\frac{n F \phi \Delta c_{A c^{-}}}{I}
$$

Where $\mathrm{F}$ is Faraday's constant $\left(96485 \mathrm{C} \cdot \mathrm{mol}^{-1}\right), \Delta \mathrm{C}_{\mathrm{Ac}}{ }^{-}$is the difference in acetate concentration in the effluent $\left(\mathrm{mol} \cdot \mathrm{L}^{-1}\right)$ as compared with the influent, $\phi$ is the total volumetric flow of influent $\left(\mathrm{L} \cdot \mathrm{s}^{-1}\right), \mathrm{n}$ is the number of electrons consumed per mol acetate produced (8), I (A) is the steady state electrical current. 


\subsection{RESULTS}

\subsubsection{Potential controlled experiments}

The three potential controlled cells with least negative cathode potential ( -560 , -630 and $-700 \mathrm{mV}$ ) showed no current and no acetate production during 34 days of operation. For this reason, these experiments were then ended and their results are not further reported on here. The fourth, most negative cathode potential (-900 $\mathrm{mV}$ ) showed increasing current during this period, and concomitantly measurable amounts of reduction products were produced. The development throughout the experiment of current, acetate concentration and partial pressures of methane, carbon dioxide and hydrogen as measured in the headspace gas composition of this last cell are displayed in Figure 2.

As can be seen in Figure 2a, during the first 44 days no other reduction products than methane were detected, with methane taking an increasingly large share in headspace composition. The cathodic current continued to increase more steeply around day 47 , accompanied by fast declining methane shares in the headspace, while the amount of hydrogen in the headspace increased. After 57 days, at which point the current reached $350 \mathrm{~mA}\left(157 \mathrm{~A} \cdot \mathrm{m}^{-2}\right.$ projected surface area), the experiment was ended because the applied voltage increased above the maximum $10 \mathrm{~V}$ output of the potentiostat. At this point, hydrogen had fully replaced the previously formed methane in the headspace and an acetate concentration of $270 \mathrm{mg} \cdot \mathrm{L}^{-1}$ was found in the electrolyte. With no longer potential or current applied to the system, acetate concentrations roughly halved at day 60 , after which no more measurements were taken.

The increase in current production, followed by a decline in the production of methane, was interpreted as follows: first (day 0 to 54), with pH levels stable at pH 6.1 throughout operation of the reactor, a steady increase in both production of current and methane suggested the progressive growth of a (presumably but not necessarily hydrogenotrophic) methanogenic community. During this period, the increase in current resulted from the increasing biological activity either by means of hydrogen-scavenging, or through the development of other, higher potential redox shuttling towards methane. After an initial lag phase typical for biological growth, this process entered an exponential phase, giving rise to the exponential increase in current (day 54 to 57). In the final stage, a depletion of $\mathrm{CO}_{2}$ prevented further production of methane, resulting in a switch towards the net production of hydrogen, which then in turn replaced the previously produced methane in the headspace. Finally, the resulting elevated hydrogen partial pressure led to a production of acetate through homoacetogenesis, which until that point had been outcompeted by hydrogenotrophic methanogenesis. 

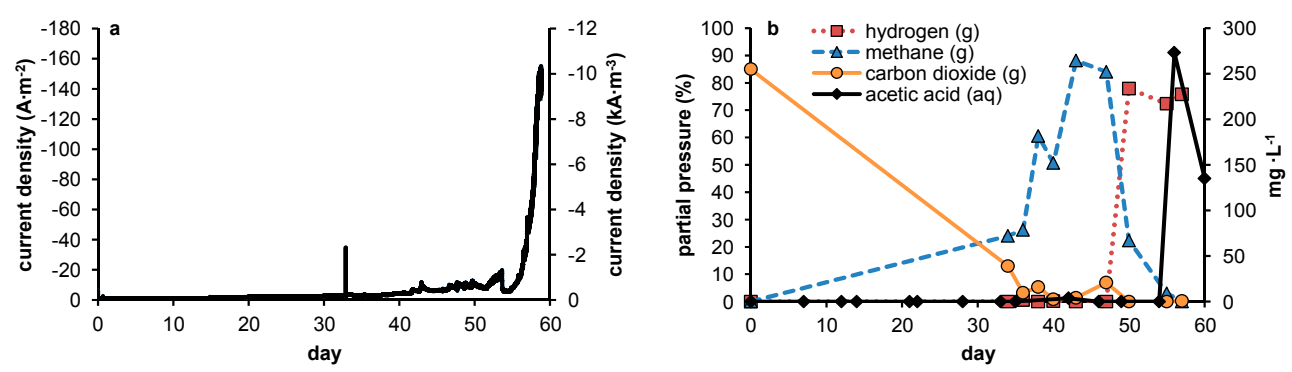

FIGURE 2 | (a) current plot of the $-900 \mathrm{mV}$ vs. $\mathrm{Ag} / \mathrm{AgCl}$ controlled biocathode throughout 57 days of operation; (b) Headspace composition throughout the experiments showed gradually increasing methane partial pressures, accompanied by decreasing levels of carbon dioxide. In the last phase of the experiment, hydrogen replaced all other gasses and subsequently acetate formation took place.

To summarize, in the four potential controlled experiments, no reduction reactions occurred at potentials above or near the equilibrium potential for hydrogen formation. Therefore, although the possibility cannot conclusively be excluded, based on the limited period of testing, the reduction of $\mathrm{CO}_{2}$ at low overpotential - thus by more direct means - seems to be unfeasible. Only for the highest chosen overpotential, formation of methane and later hydrogen and acetate was observed.

\subsubsection{Current controlled experiments}

The potential controlled experiments suggested hydrogen formation to be required for $\mathrm{CO}_{2}$ reduction under the chosen reactor conditions. Moreover, with the observation of acetate production only established at higher current densities, the effect of current density was further investigated, controlling current at four

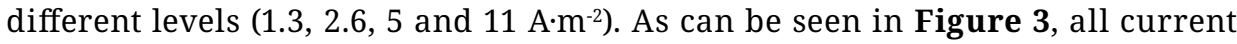
controlled cells showed production of acetate directly after start-up. In addition to the formed acetate, also hydrogen was detected in the off-gas of the reactor, even at the lowest chosen current densities.

The production of acetate with all four current densities tested showed to be unstable, reaching peak levels after 5-13 days, followed by a decline. After 19 days, none of the reactors produced measurable amounts of acetate or hydrogen, and it was assumed current was fully diverted towards the production of methane, with headspace methane partial pressures ranging from $16 \%$ for the lowest current to $55 \%$ for the highest current tested. 

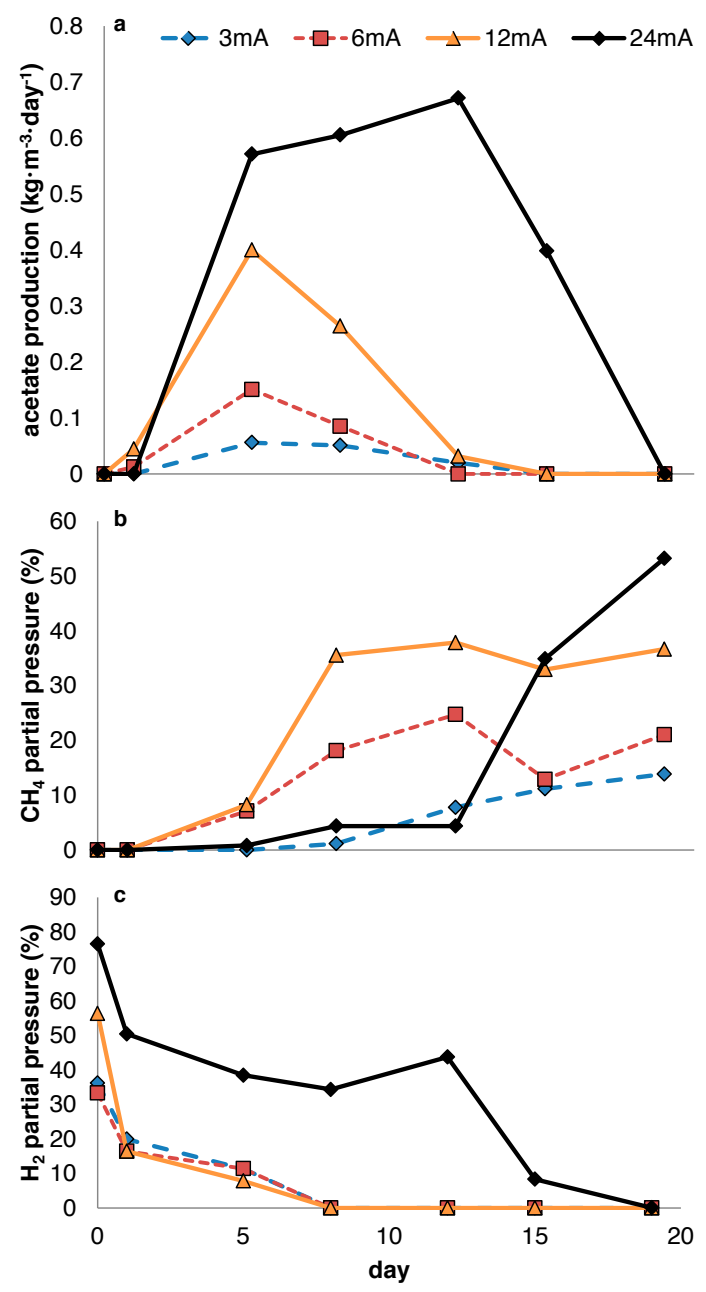

FIGURE 3 | Results obtained from four biocathodes controlled at different current densities (3, 6, 12 and $24 \mathrm{~mA}$ corresponding to 1.3, 2.6, 5 and $11 \mathrm{~A} \cdot \mathrm{m}^{-2}$ respectively): (a) Observed acetate production rates as calculated from effluent concentration measurements show a clear effect of current density, while (b) methane partial pressures and (c) hydrogen partial pressures as measured by head space gas analysis indicate progressive loss of electrons to methane as hydrogen partial pressure decreases.

Apart from reaching different peak production levels for acetate, the different currents tested showed variation in the time elapsed before peak acetate production occurred, with the highest current showing a later peak compared to lower currents. For all cells tested, headspace analysis showed increasing methane partial pressures coinciding with declining acetate levels, suggesting the decrease in acetate production was due to increasing activity of methanogens. The 
question then rises whether the observed decline in acetate production was due to competition of methanogens for hydrogen, or that - requiring the establishment of acetate production first - acetoclastic methanogenesis followed with a delay. In an attempt to gain more insight regarding this question, the effect of spiking the cells with $10 \mathrm{mM}$ of methanogen inhibitor 2-BES, after acetate production was completely extinguished, was studied (data not shown). This led to a sudden - but temporary - recovery of acetate production to similar levels of the earlier peak productions, followed by a quick decline as the inhibitor was depleted by wash-out. The almost direct resumption of acetate production upon spiking with 2-BES could indicate homoacetogens to remain active - even with net production of acetate previously being extinguished - implicating the establishment of a syntrophic interaction between homoacetogens and acetoclastic methanogens in these biocathodes. However, the option of hydrogenotrophic methanogens to be responsible for methane production could not be excluded. In the latter, a quick resumption of acetate production could be caused by resilient homoacetogens not washed out of the system. Thus, quantitative microbial community analysis would be required to be conclusive towards this point.

Figure 4 shows the production of acetate in a third round of experiments, in which the highest current density of $11 \mathrm{~A} \cdot \mathrm{m}^{-2}$ from the second experimental run was applied, but now with continuous addition of 2-BES to the catholyte. When 2-BES was used in combination with this current controlled start-up, long term stable production of acetate was achieved. Also, in these systems, after prolonged runtime, no visual change in electrolyte turbidity was observed, and measurements of suspended COD showed it to not substantially contribute to the electron balance, strongly suggesting the accumulation of biomass in the porous electrode. Moreover, gas production decreased below measurable fluxes as Coulombic efficiencies towards acetate production increased over time to reach more than $80 \%$. The latter showed the ability of the developed biomass to efficiently capture (or prevent the formation of) $\mathrm{H}_{2}$, and although partial pressures remained around $40 \%$, they still showed a decreasing trend at the end of the experiment. Remarkable was the rapid increase in cathode potential for both cells of from around $-1100 \mathrm{mV}$ to $-800 \mathrm{mV}$ in the first 10 days, followed by a steady to slightly increasing potential throughout the remaining runtime.

Although hydrogen partial pressures still reached up to $40 \%$ at day 40 , the observed increase in cathode potential could be explained by enhanced uptake of hydrogen by closely attached biofilm. Apart from hydrogen consumption by acetogenesis affecting product inhibition, the observed increase in cathode potential may also indicate some sort of catalysis, as is previously reported to occur by Jourdin and co-workers ${ }^{[8]}$. Although the nature of this catalytic phenomenon could not be further elucidated using the current methodology, in their study, Jourdin et al ascribe the catalysed formation of hydrogen in their system (followed by subsequent production of acetate in case 2-BES is added) to the biosynthesis of 
copper nanoparticles, possibly expressed by methanogens intracellularly. The reported presence of methanogens under total absence of methane production, leads to the hypothesis that these archaea live at the expense of hydrogen catalysis, thus forming an intermediate step during bioelectrochemical acetogenesis rather than a competitive one.
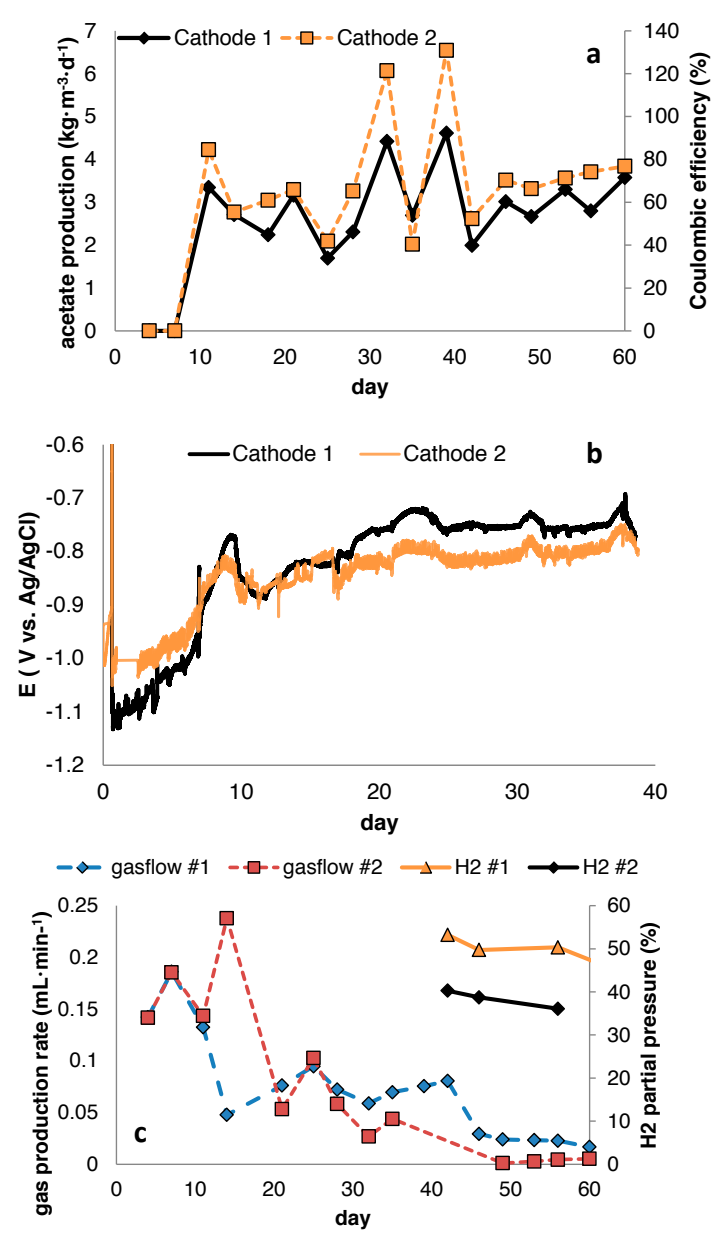

FIGURE 4 | Results obtained in duplicate current controlled cathodes operated at $24 \mathrm{~mA}\left(11 \mathrm{~A} \cdot \mathrm{m}^{-2}\right) \mathrm{con}$ taining $10 \mathrm{mM}$ of 2-BES in the electrolyte; (a) Acetate production steadily increased throughout the experimental run of 60 days, reaching nearly $4 \mathrm{~g} \cdot \mathrm{L}^{-1} \cdot \mathrm{d}^{-1}$ at a Coulombic efficiency of $80 \%$ at the end of the experiment; (b) The development of cathode potential over time displayed a steep increase at first, followed by a more stable operation around $-800 \mathrm{mV}$. (c) Starting at measurable fluxes, gas production gradually decreased for both cells - as an increasing share of current was direct towards production of acetate - and reached the detection limit by the end of the experiment. 


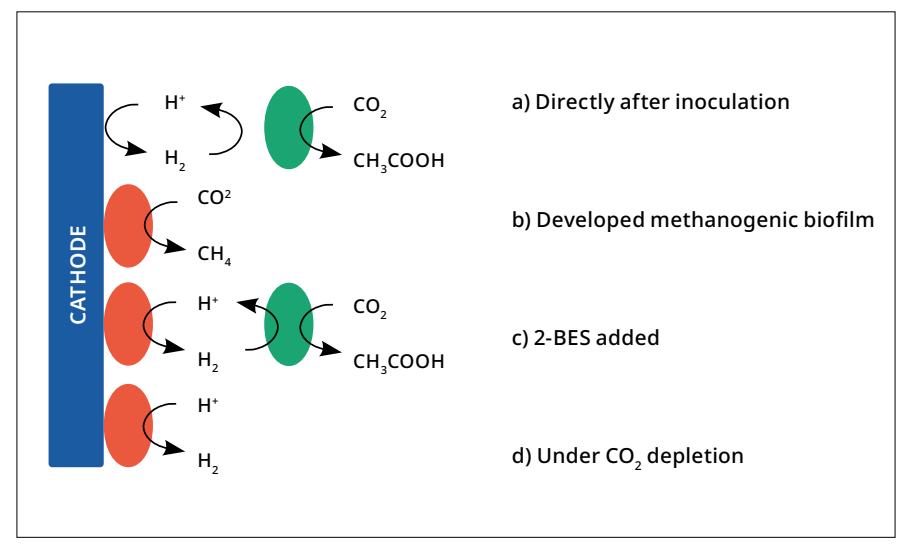

FIGURE 5 | Proposed mechanisms as occurred under tested conditions. (a) Based on the short start-up required for acetogenesis during current controlled experiments, it is likely that electron transfer during this phase does not require the establishment of a biofilm on the electrode, but is mediated by abiotically formed hydrogen. (b) The gradual shift towards production of methane observed during the later phase may be caused by the establishment of a methanogenic biofilm on the electrode, which at that point outcompetes the acetogenic community for the available substrate, rendering the acetogens effectless. (c) Upon addition of 2-BES, the methanogenic biofilm redirects electrons to form hydrogen and the produced hydrogen is consecutively used by acetogens to produce acetate. (d) In case of $\mathrm{CO}_{2}$ depletion, all electrons are shuttled to the production of hydrogen ${ }^{[8]}$.

Applying this concept of microbe-enhanced hydrogen formation to our results, this would imply that under the conditions tested, an initial acetogen-dominated microbial community was complemented by electrode-bound hydrogen producers, possibly methanogens, which initially only directed part of the current to methane. As the concentration of these bacteria increased, so did the capacity for methane formation, eventually resulting in the extinction of acetogenesis as less and less current was turned into hydrogen by the methanogens. A schematic overview of these proposed dynamics is depicted in Figure 5.

\subsection{DISCUSSION}

A well working acetate producing MES (1) has high product selectivity, (2) has high stability/robustness against contamination and/or washout and (3) does not require costive and/or toxic additives (e.g. 2-BES). In addition, from an application perspective, attention should be paid to: (1) obtaining high product concentrations, (2) high volume specific reaction rates, resulting in smaller reactor footprints and (3) high energy efficiency, producing the desired compound at the lowest energy expenses. 
In the presented research, the effect of different electrochemical control modes on both start-up and steady-state characteristics was investigated. Here, the obtained results will be discussed in relation to abovementioned aspects/criteria.

\subsubsection{Current density as control parameter for MES selectivity towards acetate}

From the perspective of reaction specificity, methanogen abundance needs to be kept as low as possible. To achieve this, reactor conditions should be so that in the steady state established, achieved specific growth rates for acetogenic organisms are as high as possible when compared to those of methanogens. For the sake of brevity, in the discussion that follows no distinction is made between hydrogenotrophic methanogenesis and homoacetogenesis followed by acetoclastic methanogenesis, as the overall reaction is identical for both.

In none of the experiments presented here in which no 2-BES was added, a steady-state was achieved in which measurable quantities of acetate were produced. The experimental conditions thus rendered ineffective in outcompeting methanogens sufficiently during steady state reactor operation. However, during the start-up of current controlled experiments, acetate production and the duration thereof was positively related to current density. In potential controlled
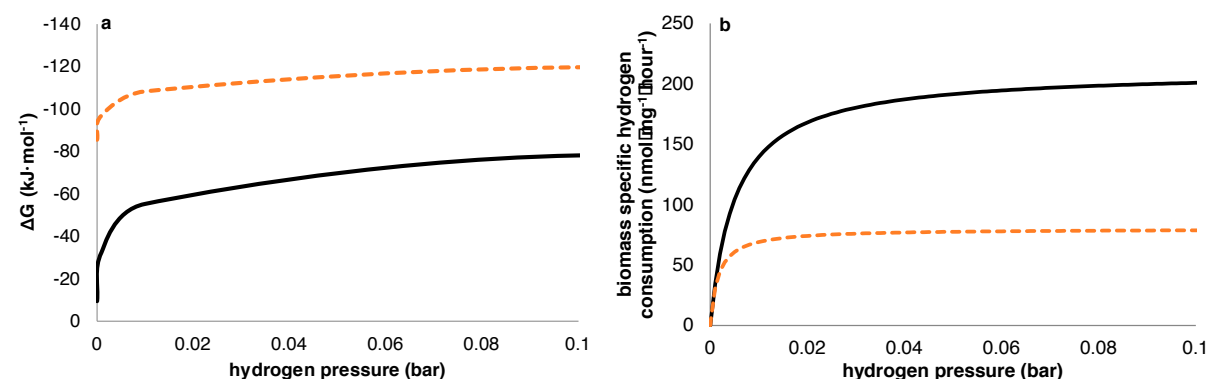

FIGURE 6 | Shows the effect of variable hydrogen pressures on the competition between acetogens (black, solid line) and methanogens (orange, dashed line) in terms of (a) Gibbs free energy of reaction ${ }^{[12]}$ and (b) biomass specific substrate consumption rate (Monod parameters for modeling growth were taken from reported values in Kotsyurbenko et $\mathrm{al}^{[21]}$ ). As can be seen methanogens have a thermodynamic advantage over homoacetogens throughout the whole domain. This thermodynamic advantage is reflected in the growth curves through the hydrogen affinity $\left(\mathrm{K}_{\mathrm{s}}\right)$ for methanogens in general being lower than for acetogens. However, acetogenesis achieves higher conversion rates at higher hydrogen partial pressures $\left(\mu_{\max }\right)$ compared to methanogenesis, rendering hydrogen pressure an essential parameter to steer competition between acetogens and methanogens ${ }^{[21]}$. 
experiments, net acetate production only occurred when current increased rapidly, thus clearly not reflecting a steady-state. As was already stated in the introduction (Table 1), from a thermodynamic perspective, methanogens can gain more energy than acetogens from the consumption of hydrogen and $\mathrm{CO}_{2}$. Figure 6a shows this thermodynamic advantage for methanogens is valid at any hydrogen pressure. Our observations however suggest that - under the conditions chosen - a kinetic difference exists between the acetogens and methanogens, both present in the used inoculum, resulting in a temporary higher net growth rate for acetogens during start-up, despite the higher thermodynamic available energy for methanogens. Such differences in growth kinetics have been described previously for methanogens and acetogens using hydrogen as common electron donor ${ }^{[2,21]}$, and to allow comparison with the thermodynamic equilibria from Figure 6a, Figure $\mathbf{6 b}$ illustrates biomass specific substrate consumption rates resulting at different hydrogen pressures. As substrate consumption is directly related to growth via yield, this shows homoacetogens thrive better than methanogens at higher hydrogen partial pressures.

As in our experiments the production of acetate was always accompanied by elevated hydrogen partial pressures, we presume electron transport from electrode to acetate to be primarily mediated through hydrogen. As such, the relative growth rates of both methanogens and acetogens were directly affected by the hydrogen partial pressure, in turn resulting from the steady-state obtained by, on the one hand, production of hydrogen by the set current density and, on the other hand, its consumption by microbial activity. At this point, little is known about the relationship between current density and local hydrogen partial pressures at the electrode, and this is likely to be specific for different electrode materials, depending on how accessible the material is to biofilm formation (e.g. hydrophobicity, roughness, decorated by functional groups etc.). Therefore, rather than predicting an optimal current density based on reported growth parameters, a more empirical approach towards optimization of current density with relation to biocathode selectivity will be required. This is the case both when hydrogen shows an inevitable intermediate in biocathodic production of acetate - as too high current density will result in loss of selectivity by evading hydrogen gas - and in case alternative mechanisms turn out to play a role in electron transfer from electrode to microbe: the uptake of molecular hydrogen by both acetogens and methanogens is mediated by ferredoxin reducing hydrogenases ${ }^{[2,23]}$, a group of enzymes known to possess typically high turnover rates ${ }^{[24]}$. Given the mechanistic similarities and characteristics occurring at the start of both pathways ${ }^{[25,26]}$, the kinetic advantage of acetogens compared to methanogens at higher hydrogen pressures is probably due to a conversion further down the electron transfer chain (ETC) of methanogens becoming rate limiting under these conditions. In contrast, at low hydrogen pressures, the first electron step (thus from hydrogen to the ETCs initial electron acceptor) in acetogens is likely to be thermodynamically controlled 
(instead of kinetically). The observation that the rate-limiting step for methane formation occurs further down the ETC is of importance, as it makes it more likely that this limitation will remain in case of an alternative mechanism of electron transfer between cathode and microbe. This would give the homoacetogens a competitive advantage over methanogens at higher current densities regardless the exact nature of electron transfer between electrode and microbe. Therefore, we pose current density to be a defining parameter for selectivity in acetate producing MES.

\subsubsection{Considerations on the effect of current density on overall reactor performance}

As previously stated, selectivity is not the only criterion defining reactor performance in MES, and it therefore holds relevance to further delineate the effects current density presumably has on product concentration, reaction rates and energy efficiency.

In terms of volume specific reaction rates, the application of high current densities is attractive as it drives acetogens close to their maximum turnover rates. In addition to current density, volume specific reaction rates may be influenced through control of HRT and/or SRT. In case of planktonic cells, both HRT and SRT are the same. In case of a biofilm attached to the cathode the two can be uncoupled and separately controlled. In case the majority of the conversion is being done by planktonic cells, a longer HRT (and thus SRT) will positively impact biomass density, and therefore result in higher volume specific conversion rates and product concentrations. Assuming that a biofilm forms on the electrode surface, HRT and SRT are uncoupled, and it becomes crucial to use an electrode material with high specific surface area (with pores accessible to microorganisms) to achieve high biofilm density and high volumetric acetate production rates provided that methanogenesis can be kept to low levels. To this end, one has to bear in mind that a trade-off will show between biomass retention time and product selectivity, as also methanogens will be washed out slower and may win competition if conditions are suitable (Figure 6). In case of biofilm formation, the SRT/HRT ratio can be tuned e.g. by inducing various levels of sheer stress on the biofilm, for instance by recirculation at different flowrates, and an optimum with respect to process selectivity might be sought.

Inherent to higher current densities is also the occurrence of higher overpotential, and thus lower voltage efficiency. At the same time, higher current density leads to higher Coulombic efficiency towards acetate. Thus, a compromise has to be found between selectivity and voltage efficiency to obtain optimal overall energy efficiency. Also to this end, the use of high specific surface area electrode materials will be beneficial, as it will lower the overpotential. Additionally, the 
observation of a drop in overpotential both for cathodes with and without addition of 2-BES in the presented results, showed that the application of higher current densities in these systems not necessarily has to result in extensive energetic losses, even if hydrogen is functioning as intermediate.

To summarize, we hypothesize that, in order for an acetate producing cathode to perform well both in terms of product selectivity, production rate and obtained product concentrations, a high specific surface area electrode material will have to be used at a high current density allowing a (relatively) high biomass density at a (relatively) low biomass retention time. Ideally, conversions are taking place in a cathode attached biofilm, thus allowing a controllable SRT/HRT ratio so that a good balance can be found between the obtained biomass density and its SRT, while optimizing HRT to get a good product concentration. The extent to which aforementioned aspects are intertwined are yet to be determined and quantitative reporting of the effect of current density on product concentration, energy efficiency and specific reaction rates therefore deserves recommendation for future research in MES.

\subsubsection{Other factors affecting (Monod) growth characteristics of methanogens and acetogens}

The Monod parameters used to describe specific growth rates in relation to hydrogen pressure vary from species to species both for methanogens and homoacetogens ${ }^{[27]}$. By working with pre-enriched inocula, such interspecies variation could be taken advantage of, put aside the vulnerability this strategy has towards contamination ${ }^{[17]}$. Apart from interspecies variation in growth characteristics, the growth parameters are known to significantly alter within a single species in response to certain environmental conditions not directly involved in metabolism, most importantly temperature, $\mathrm{pH}$ and salinity, as a change in these variables directly affects the energy budget of the organism available for growth ${ }^{[21]}$. The extent of these impacts may differ from species to species, and it therefore holds interest to review whether operational parameters other than current density may be identified that may favour growth of acetogens over methanogens when controlled adequately. To this end, three main parameters are discussed: (1) pH and closely related, salinity, (2) temperature and (3) the presence or absence of specific co-factors / inhibiting compounds.

Regarding $\mathrm{pH}$, consensus is hard to be found based on earlier reports in literature. Studies report a competitive advantage for acetogens both at low ${ }^{[15,28]}$ and high $\mathrm{pH}^{[11]}$. These observations seem counteracted by reports on methanogens thriving both in extreme acidic ${ }^{[29]}$ and alkaline ${ }^{[30]}$ environments. The enhanced growth of acetogens in highly alkaline media has been related to faster growth at increased concentrations of $\mathrm{CO}_{2}{ }^{[11]}$. However, although the concentration of 
$\mathrm{CO}_{2}$ affects Gibbs free energy of acetogenesis more than methanogenesis (due to reaction stoichiometry), extremely low $\mathrm{K}_{\mathrm{s}}$ values typically reported towards this compound, in relation to high solubility for this gas in neutral media, make this theory rather questionable ${ }^{[31]}$. Alternative explanations for the variation in $\mathrm{pH}$ related growth rate variation are inhibition by acetate ${ }^{[20]}$ or the presence/ absence of specialized membrane pumps ${ }^{[32]}$, the latter of which may also explain differences in salinity tolerance between species. However, based on the available data from literature, neither $\mathrm{pH}$ nor salinity may be used as an unambiguous parameter to steer selectivity. That said, higher salinities and alkalinity do provide advantages in terms of ionic conductivity and $\mathrm{CO}_{2}$ solubility, potentially improving both energy efficiency and final product concentrations.

Regarding temperature effects on competition between methanogens and acetogens, reports on tundra ecosystems show a relative increase in growth rates for acetogens compared to methanogens at temperatures between 4 and $10{ }^{\circ} \mathrm{C}{ }^{[21]}$. However, at these temperatures maximum conversion rates for the studied acetogens are also reduced by more than 3 fold compared to mesophilic temperature operation, meaning that operating a reactor under psychrophilic conditions would put serious restrictions to the volume specific reaction rate.

Microbes are complex catalysts, in general shuttling electrons from substrate to product through an electron transfer chain consisting of a variety of enzymatic complexes often containing specific metallic compounds at their active site. By either restricting the production of specific parts of such an ETC, for instance by limiting the supply of essential cofactors, or by inhibiting their functioning by selective blocking - as is the case with 2-BES, substantial increases in specificity towards acetogenesis might be obtained. However, apart from the fact that more detailed knowledge would be required to identify such selective cofactors or possible blocking compounds, it is questionable whether this strategy would ever find its useful application on a relevant scale: cofactors are normally needed only in trace amounts, thus extreme purity of used electrolytes would be required in order to make such a strategy effective, putting a costly requirement on its composition. The addition of chemicals is, apart from costly, seldom acceptable from environmental perspectives, as is the case with the bromide containing 2-BES.

\subsection{CONCLUSIONS}

We identify current density as a most promising operational parameter for optimizing acetate producing biocathodes, rather than optimization of $\mathrm{pH}$ or temperature. Regarding the latter two, we suggest mesophilic operation and mildly alkaline conditions to allow for sufficient solubility of the main substrate, $\mathrm{CO}_{2}$, while avoiding excessive overpotentials due to the proton dependency of acetogenesis. Future experimental efforts should be directed towards a 
more quantitative investigation of current density and its relation to reactor performance. Therefore, in our opinion, acetate producing cathodes may be better controlled at a set current, rather than at a controlled potential. The here presented data shows that start-up strategy is important for reactor performance in acetate producing cathodes. Within the experimental periods tested, controlled current showed to be more successful than controlled potential in establishing the production of acetate by reduction of $\mathrm{CO}_{2}$. However, when testing higher current densities a mixed approach might be advised, starting with either potential control or moderate current density to allow biofilm formation while preventing ablation by hydrogen bubble formation, followed by current control at the desired current density, ensuring efficient electron shuttling towards acetate once sufficient biomass has formed to prevent bubble formation. Closely related to current density, a primary role is foreseen regarding the used electrode materials, as it forms the most important design variable in a biocathode and determines to a large extent - amongst others aspects - biomass attachment/retention specifics and electrode specific surface area. We argue that by optimizing reactor control by these means, neither extensive pre-enrichment of biota nor selective inhibition of methanogenesis by means of 2-BES is required to obtain selective acetate production at high reaction rates and concentrations in the future.

\subsection{ACKNOWLEDGEMENTS}

This work was performed in the cooperation framework of Wetsus, European Centre of Excellence for Sustainable Water Technology (www.wetsus.eu). Wetsus is co-funded by the Dutch Ministry of Economic Affairs and Ministry of Infrastructure and Environment, the Province of Fryslân, and the Northern Netherlands Provinces. The authors like to thank the participants of the Resource Recovery research theme for the fruitful discussions and their financial support. Additionally, the authors would like to thank Sanne Raes for her structuring view and Sonja Willemse for extensive reviewing of the manuscript. 


\subsection{REFERENCES}

1. Rabaey, K. \& Rozendal, R. A. Microbial electrosynthesis - revisiting the electrical route for microbial production. Nat. Rev. Microbiol. 8, 706-16 (2010).

2. Chabert, N., Amin Ali, O. \& Achouak, W. All ecosystems potentially host electrogenic bacteria. Bioelectrochemistry 106, 88-96 (2015).

3. Nevin, K. P. et al. Electrosynthesis of organic compounds from carbon dioxide is catalyzed by a diversity of acetogenic microorganisms. Appl. Environ. Microbiol. 77, 2882-6 (2011).

4. Rabaey, K., Girguis, P. \& Nielsen, L. K. Metabolic and practical considerations on microbial electrosynthesis. Curr. Opin. Biotechnol. 22, 371-377 (2011).

5. Lovley, D. R. \& Nevin, K. P. Electrobiocommodities: Powering microbial production of fuels and commodity chemicals from carbon dioxide with electricity. Curr. Opin. Biotechnol. 24, 385-390 (2013).

6. Marshall, C. W., Ross, D. E., Fichot, E. B., Norman, R. S. \& May, H. D. Long-term Operation of Microbial Electrosynthesis Systems Improves Acetate Production by Autotrophic Microbiomes. Environ. Sci. Technol. 47, 6023-9 (2013).

7. Jourdin, L., Freguia, S., Flexer, V. \& Keller, J. Bringing High-Rate, CO2-Based Microbial Electrosynthesis Closer to Practical Implementation through Improved Electrode Design and Operating Conditions. Environ. Sci. Technol. 50, 1982-1989 (2016).

8. Jourdin, L., Lu, Y., Flexer, V., Keller, J. \& Freguia, S. Biologically Induced Hydrogen Production Drives High Rate/High Efficiency Microbial Electrosynthesis of Acetate from Carbon Dioxide. ChemElectroChem 3, 581-591 (2016).

9. Jourdin, L. et al. High Acetic Acid Production Rate Obtained by Microbial Electrosynthesis from Carbon Dioxide. Environ. Sci. Technol. 49, 13566-13574 (2015).

10. Blanchet, E. M. et al. Importance of the hydrogen route in up-scaling electrosynthesis for microbial CO2 reduction. Energy Environ. Sci. 3731-3744 (2015). doi:10.1039/C5EE03088A

11. Annie Modestra, J., Navaneeth, B. \& Venkata Mohan, S. Bio-electrocatalytic reduction of CO2: Enrichment of homoacetogens and $\mathrm{pH}$ optimization towards enhancement of carboxylic acids biosynthesis. J. CO2 Util. 10, 78-87 (2015).

12. Thauer, R. K., Jungermann, K. \& Decker, K. Energy conservation in chemotrophic anaerobic bacteria. Bacteriol. Rev. 41, 100-180 (1977).

13. Liu, D., Zhang, L., Chen, S., Buisman, C. \& Ter Heijne, A. Bioelectrochemical enhancement of methane production in low temperature anaerobic digestion at $10^{\circ} \mathrm{C}$. Water Res. 99, 281-287 (2016).

14. Geppert, F. et al. Bioelectrochemical Power-to-Gas: State of the Art and Future Perspectives. Trends Biotechnol. 34, 879-894 (2016).

15. Gildemyn, S. et al. Integrated Production, Extraction, and Concentration of Acetic Acid from CO 2 through Microbial Electrosynthesis. Environ. Sci. Technol. Lett. 2, 325-328 (2015).

16. Zhang, T. et al. Improved cathode materials for microbial electrosynthesis. Energy Environ. Sci. 6, 217 (2013).

17. Patil, S. A. et al. Selective Enrichment Establishes a Stable Performing Community for Microbial Electrosynthesis of Acetate from CO2. Environ. Sci. Technol. 49, 8833-8843 (2015). 
18. Nevin, K. P., Woodard, T. L. \& Franks, A. E. Microbial Electrosynthesis: Feeding Microbial Electrosynthesis : Feeding Microbes Electricity To Convert Carbon Dioxide and Water to Multicarbon Extracellular Organic. Am. Soc. Microbiol. 1, 1-4 (2010).

19. Bouwer, E. J. \& McCarty, P. L. Effects of 2-Bromoethanesulfonic Acid and 2-Chloroethanesulfonic Acid on Acetate Utilization in a Continuous-Flow Methanogenic Fixed-Film Column. Appl. Envir. Microbiol. 45, 1408-1410 (1983).

20. Vavilin, V. A., Lokshina, L. Y., Rytov, S. V., Kotsyurbenko, O. R. \& Nozhevnikova, A. N. Description of twostep kinetics in methane formation during psychrophilic $\mathrm{H} 2 / \mathrm{CO} 2$ and mesophilic glucose conversions. Bioresour. Technol. 71, 195-209 (2000).

21. Kotsyurbenko, O. R., Glagolev, M. V., Nozhevnikova, A. N. \& Conrad, R. Competition between homoacetogenic bacteria and methanogenic archaea for hydrogen at low temperature. FEMS Microbiol. Ecol. 38, 153-159 (2001).

22. Martin, W. F. Hydrogen, metals, bifurcating electrons, and proton gradients: The early evolution of biological energy conservation. FEBS Lett. 586, 485-493 (2012).

23. Liu, Y. \& Whitman, W. B. Metabolic, phylogenetic, and ecological diversity of the methanogenic archaea. Ann. N. Y. Acad. Sci. 1125, 171-189 (2008).

24. Jones, A. K., Sillery, E., Albracht, S. P. J. \& Armstrong, F. A. Direct comparison of the electrocatalytic oxidation of hydrogen by an enzyme and a platinum catalyst. Chem. Commun. 866-867 (2002). doi:10.1039/b201337a

25. Ragsdale, S. W. \& Pierce, E. Acetogenesis and the Wood-Ljungdahl Pathway of CO2 Fixation. 1784, 1873-1898 (2009).

26. Stupperich, E., Hammel, K. E., Fuchs, G. \& Thauer, R. K. Carbon monoxide fixation into the carboxyl group of acetyl coenzyme A during autotrophic growth of Methanobacterium. FEBS Lett. 152, 21-23 (1983).

27. Cord-Ruwisch, R., Seitz, H. J. \& Conrad, R. The capacity of hydrogenotrophic anaerobic bacteria to compete for traces of hydrogen depends on the redox potential of the terminal electron acceptor. Arch. Microbiol. 149, 350-357 (1988).

28. LaBelle, E. V., Marshall, C. W., Gilbert, J. A. \& May, H. D. Influence of acidic pH on hydrogen and acetate production by an electrosynthetic microbiome. PLoS One 9, e109935 (2014).

29. Kotsyurbenko, O. R. et al. Shift from acetoclastic to H2-dependent methanogenesis in a West Siberian peat bog at low pH values and isolation of an acidophilic Methanobacterium strain. Appl. Environ. Microbiol. 73, 2344-2348 (2007).

30. Mathrani, I. M., Boone, D. R., Mah, R. A., Fox, G. E. \& Lau, P. P. Methanohalophilus zhilinae sp. nov., an Alkaliphilic, Halophilic, Metholotrophic Methanogen. Int. J. Syst. Bacteriol. 38, 139-142 (1988).

31. Braun, K. \& Gottschalk, G. Effect of molecular hydrogen and carbon dioxide on chemo-organotrophic growth of Acetobacterium woodii and Clostridium aceticum. Arch. Microbiol. 128, 294-298 (1981).

32. Rinzema, A., van Lier, J. \& Lettinga, G. Sodium inhibition of acetoclastic methanogens in granular sludge from a UASB reactor. Enzyme Microb. Technol. 10, 24-32 (1988).

33. Molenaar, S. D., Mol, A. R., Sleutels, T. H. J. A. \& Heijne, A. Microbial Rechargeable Battery: Energy Storage and Recovery through Acetate. Environ. Sci. Technol. Lett. 3, 144-1 (2016).

34. Zehnder, A. J. B., Huser, B. A., Brock, T. D. \& Wuhrmann, K. Characterization of an acetatedecarboxylating, non-hydrogen-oxidizing methane bacterium. Arch. Microbiol. 124, 1-11 (1980). 



\section{IN SITU BIOFILM QUANTIFICATION IN BIOELECTROCHEMICAL SYSTEMS USING OPTICAL COHERENCE TOMOGRAPHY}

Detailed studying of microbial growth in bioelectrochemical systems is required for their proper design and operation. This chapter describes the use of Optical Coherence Tomography (OCT) as a tool for in situ and non-invasive quantification of biofilm growth on electrodes (bioanodes). An experimental platform is designed and described in which transparent electrodes are used to allow for real-time, three-dimensional biofilm imaging. The accuracy and precision of the developed method is assessed by relating OCT results to well-established standards for biofilm quantification (COD and Total N) and show high correspondence to these standards. Biofilm thickness as observed by OCT ranged between 3 and $90 \mu \mathrm{m}$ for experimental durations ranging from 1 to 24 days. This translated to growth yields between 38 and $42 \mathrm{mg} \mathrm{COD} \operatorname{comass}_{\text {biog }} / \mathrm{g}$ $\mathrm{COD}_{\text {acetate }}$ at an anode potential of $-0.35 \mathrm{~V}$ vs. Ag/AgCl. Time-lapse observations of an experimental run performed in duplicate show high reproducibility in obtained microbial growth yield using the developed method. As such, OCT is identified as a powerful tool for conducting in-depth characterizations of microbial growth dynamics in BESs. Additionally, the presented platform allows concomitant application of this method with various optical and electrochemical techniques.

This chapter has been published as: Molenaar, S.D.; Sleutels, T.; Pereira, J.; Iorio, M.; Borsje, C.; Zamudio, J.A.; Fabregat-Santiago, F.; Buisman, C.J.N.; ter Heijne, A., In situ biofilm quantification in Bioelectrochemical Systems using Optical Coherence Tomography, ChemSusChem, 2018, 11, 13, 2171-2178, 


\subsection{INTRODUCTION}

Microorganisms play a pivotal role in bioelectrochemical systems (BESs). In BESs, microorganisms catalyse electrochemical formation or degradation of organic molecules by interfacing their biological metabolism with an electrode ${ }^{[1-3]}$. The interaction between microorganisms and electrical conductive structures makes these systems unique and versatile, with potential applications in electrical energy storage ${ }^{[4,5]}$, energy- and nutrient-recovering wastewater treatment systems $\mathrm{s}^{[6-8]}$, production of high value chemical commodities ${ }^{[9,10]}$, long-term off-grid low-power electricity generation ${ }^{[11,12]}$ and the development of highly specific and innovative biosensors ${ }^{[13,14]}$. Additionally, microorganisms may provide temporal charge storage within the microbial cell ${ }^{[15-17]}$. From a scientific perspective, BESs may function as a platform for fundamental microbiological studies ${ }^{[18-20]}$, as unique metabolic properties ${ }^{[21]}$ can now be studied using a plethora of electrochemical analyses ${ }^{[22-24]}$.

For all current and future foreseen applications of BESs, further optimisation is needed before they may become economically viable ${ }^{[25,26]}$. With microorganisms functioning as catalysts in BESs, their growth and activity is inextricably linked to the performance of these systems. Biomass growth and activity in BESs is known to be dynamic and highly adaptive to changing operational conditions ${ }^{[27,28]}$. Although biomass growth and activity has been studied using different techniques ${ }^{[29,30]}$, little quantitative information is available regarding the relation between specific growth rates, activities and biomass yields at one hand, and operational conditions (e.g. electrode potential, substrate availability or shear stress) at the other.

For a systematic exploration of the effects of operational parameters on biomass development and, ultimately, system performance [31], mathematical modelling and model validation provide useful tools ${ }^{[32-34]}$. A major threshold however hindering further calibration and validation of currently available models, concerns adequate monitoring of the catalyst loading. Unlike in abiotic electrochemistry, where this is mostly a pre-defined and applied quantity, catalyst loading is a dynamic variable in BESs, as the amount of biomass (and activity thereof) is constantly changing as a result of operational conditions throughout the experiment. Together with the great efforts and time investments involved in construction, start-up and operation of BESs, this creates the requirement for a non-invasive method for continuous biomass monitoring in these systems, during an experiment.

In practice, the challenge of measuring biomass quantities in BESs focuses on the measurement of (electroactive) biofilms. High current densities in BESs seems to occur exclusively in situations where immobilisation of microorganisms on the electrode takes place, by means of biofilm formation ${ }^{[35-38]}$. With low amounts of suspended biomass repeatedly reported in high performing BESs ${ }^{[39,40]}$, biofilms form the largest presence of (active) biomass within these systems. 
In the last decades, several methods have been explored to investigate biofilm form, size and structure, ranging from traditional colony forming unit (CFU) counting to super-resolution fluorescent imaging techniques ${ }^{[41-43]}$. However, these methods described so far are either invasive or do not allow for assessment of global morphological properties. For instance, while confocal laser scanning microscopy (CLSM) ${ }^{[44]}$ achieves excellent sub-micron resolution and can be applied to a wide field of view ${ }^{[45]}$, it lacks sufficient penetration depth (max. $200 \mu \mathrm{m}$ in opaque biological samples, but considerably less in chromophorecontaining tissues), thus often not allowing for accurate volumetric analysis ${ }^{[41,45,46]}$. Enhancements regarding penetration depth can be made by using two-photon excitation microscopy (TPE) however this requires staining of the biofilm ${ }^{[47]}$. Moreover, more conventional light microscopy methods as phase contrast/phase modulation ${ }^{[48,49]}$, epifluorescence ${ }^{[50]}$ and differential interference microscopy, when applied by means of optical slicing, can hardly attain acceptable image contrast at penetration depths $>200 \mu \mathrm{m}$, while at lesser spatial resolution compared to CLSM $^{[51]}$. Quartz crystal microbalancing (QCM) has been successfully used in BESs for accurate balancing of biota on a microelectrode, but severely restricts cell design and size while not providing spatial distribution data of the biofilm ${ }^{[22]}$. Scanning transmission X-ray microscopy (STXM) and scanning/transmission electron microscopy (SEM/TEM) have been applied to reveal high-resolution information about sub-micron structure and composition of biofilms, but require fixation or cryogenic preparation of samples ${ }^{[45,53]}$. Confocal Raman microscopy (CRM) has been employed for non-destructive, three-dimensional characterisation of the biofilm structure and composition, but requires extensive spectral library build-up. Moreover, CRM is prone to excessive acquisition times when used to study larger morphologies, being a line scan method with a reported pixel integration time of $0.2 \mathrm{~s}^{[54]}$.

Here, we present a novel method for monitoring anodic biofilm dynamics and quantification of biomass using Optical Coherence Tomography (OCT) as imaging technique. OCT is a technique that allows the acquisition of three-dimensional images from optical scattering media at a micrometre resolution ${ }^{[55,56]}$. OCT, being a form of quantitative phase contrast imaging, is conventionally used within the medical field of ophthalmology where it is routinely applied for the determination of the thickness and dimensions of the cornea ${ }^{[57]}$. As a result, these devices are readily commercially available, intuitively operated by the aid of well-designed software, and at expenses considerably lower than that of a CLSM apparatus. As a scanning method using relatively low energy light (in the visible range), it tolerates a relatively large operational distance to the object to be sampled. In combination with the visible light causing little to none degeneration on biological tissues, it can be applied in-line for continuous observations while still allowing considerable flexibility in electrochemical cell design and geometry. By combining such an optical technique with electrochemical analyses, quantitative data with high 
temporal resolution can be obtained. The use of such data for future calibration and validation of biomass growth models is anticipated, and can be expected to reveal new insights in the intertwined processes occurring in BESs.

We have implemented and validated the use of OCT to determine the three-dimensional coverage and volume of the electro-active biofilm growing on a flat anode surface. For this purpose, we have adapted a BES to include an optical transparent anode, allowing in-situ analysis of the biofilm. The anodes used are glass plates, coated with a thin layer of fluorine-doped tin oxide (FTO). These electrodes are (i) transparent, which enables visualisation techniques to quantify biomass; (ii) conductive and chemically stable, which allows for biofilm growth and current density production similar to other well performing flat plate electrodes used in BESs ${ }^{[58,59]}$; (iii) extremely flat, which gives a well-defined surface area suitable for both biofilm characterisation and visualisation techniques and (iv) allows for electrochemical characterisation techniques.

To assess and validate whether OCT can be used as an accurate and precise tool for quantification of biomass density, we conducted OCT measurements on 24 independent experiments, and compared these with data obtained by sacrificial biomass quantification. This demonstrated OCT as a reliable tool for in-situ biomass quantification under the conditions tested. To assess whether OCT could also be used as a non-invasive tool for determining biomass growth and activity, repeated measurements were done during two subsequent experiments, in which the development of biomass was tracked over time.

\subsection{EXPERIMENTAL PROCEDURES}

\subsubsection{Reactor setup}

Electrochemical cells consisted of two Plexiglas plates with flow channels $\left(33 \mathrm{~cm}^{3}\right)$ separated by a bipolar membrane (Ralex® PE-BPM, MEGA a.s., Czech Republic). FTO-coated glass slides $\left(22.3 \mathrm{~cm}^{2}\right.$ exposed anode area) were used as anodes for biofilm to attach to and grow on. Anode current collectors were made of graphite paper sheets, from which the flow channel area was extruded. By extruding the flow channel from the current collector, the graphite paper was touching the FTO layer only in places where no electrolyte was present. A flat sheet of stainless steel (316 alloy) lined with a sheet of carbon paper was used as a cathode. Figure 1 shows a photographic overview of the constructed cells. Ag/AgCl reference electrodes (Prosense, Oosterhout, The Netherlands; $+0.203 \mathrm{~V}$ vs standard hydrogen electrode) were inserted into the anode chamber via a Haber-Luggin capillary filled with a saturated solution of $\mathrm{KCl}$ and all potentials are expressed with respect to this electrode. 


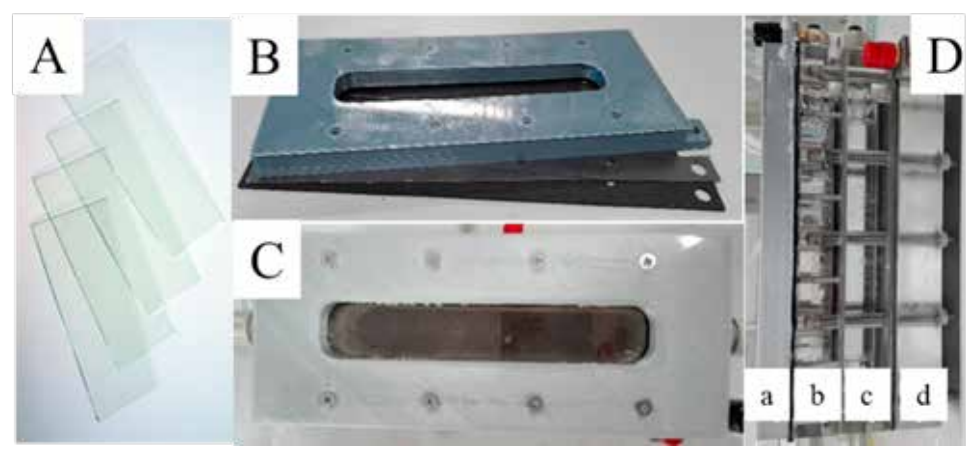

FIGURE 1 | Photographic overview of used cell component and their assembly showing (A) FTO electrodes, (B) anode end plate, graphite current collector and neoprene gasket (from top to bottom), (C) top view of the anode compartment with exposed face of FTO electrode in the middle, (D) completely assembled cell with clearly visible: (a) anode end-plate, (b) anode flow compartment, (c) cathode flow compartment, (d) cathode end-plate.

\subsubsection{Reactor inoculation and operation}

Reactors were inoculated with biomass from active acetate oxidising bioanodes. Influent consisted of (g/L): $0.74 \mathrm{KCl}, 0.58 \mathrm{NaCl}, 3.4 \mathrm{KH}_{2} \mathrm{PO}_{4}, 4.35 \mathrm{~K}_{2} \mathrm{HPO}_{4}, 0.28$ $\mathrm{NH}_{4} \mathrm{Cl}, 0.1 \mathrm{MgSO}_{4} .7 \mathrm{H}_{2} \mathrm{O}, 0.1 \mathrm{CaCl}_{2} .2 \mathrm{H}_{2} \mathrm{O}, 0.82$ Sodium acetate, 2.1 Sodium 2-Bromoethanesulfonate, $1 \mathrm{~mL}$ trace metal mixture and $1 \mathrm{~mL}$ vitamins mixture according to DSMZ culture medium $141^{[64]}$.The influent stock solution was continuously sparged with $\mathrm{N}_{2}$ before being fed to the reactor to maintain anaerobic conditions. The influent was fed to the reactor at a rate of $0.16 \mathrm{~mL} / \mathrm{min}$ (HRT $=20$ $\mathrm{h}$, total recirculated anolyte volume $=200 \mathrm{~mL}$ ). Catholyte initially consisted of $10 \mathrm{mM}$ phosphate buffer at $\mathrm{pH}$ 7, which was not replaced between experimental runs. Catholyte was continuously sparged with $\mathrm{N}_{2}$ to strip it from any formed hydrogen gas.

\subsubsection{Experimental design}

The use of OCT for biofilm quantification was validated by conducting a total of 24 runs, where each run started with a clean FTO anode. The anode potential during each run was controlled at $-350 \mathrm{mV}$ with a potentiostat (N-stat D-module, Ivium Technology, The Netherlands). In order to bring variation to the amount of biofilm at sampling, a temporally equally spaced sampling schedule was chosen in which runs produced current between 1 up to 24 days before sampling. Just 
before the end of a run, reactors were assessed by OCT. They were then dismantled and biofilm was harvested for further chemical analyses.

The use of OCT for non-invasive time-lapse observations of biofilm development was tested and illustrated. To this end, two cells were constructed and operated as above, but instead of being harvested after OCT, these cells were reconnected and their operation was continued. Moreover, regarding the influent for these systems, acetate was separated from other nutrients to improve substrate loading stability. In order to analyse these systems by OCT, they were disconnected both hydraulically and electronically for approximately two hours each time the system was sampled. Sampling periodicity was defined pragmatically, aiming for a comparable amount of charge to have passed between measurement points. In total, this practice was carried during 14 days for one, and 17 days for the other cell.

\subsubsection{OCT acquisition and image processing}

In-situ imaging of the FTO anode was performed using a spectral domain optical coherence tomograph (Thorlabs Ganymede SD-OCT System). To get a representative dataset regarding the distribution of biofilm on the electrode, the OCT measurement was performed at 54 predefined, evenly distributed spots (see Figure 2), using an automated translation stage (Thorlabs LTS300\&150/M). The axial (i.e. perpendicular to the anode) spatial resolution for the OCT instrument is below $5.8 \mu \mathrm{m}$, and the lateral (i.e. parallel to the electrode) resolution is $8 \mu \mathrm{m}$. The OCT was fitted with a $5 \mathrm{x}$ telecentric scan lens (Thorlabs LSM03BB), configured to make a "B-scan" (horizontal line scan) consisting of 1000 equally spaced "A-scans" (depth scans) over a 1 $\mathrm{mm}$ transect using the instruments software. See Figure 2 for a schematic overview of the acquisition method used. Based on these scan settings and on an assumed bulk refractive index of 1.33 (identical to that of water), twodimensional cross-sectional images were acquired of max $2.1 \mathrm{~mm}$ deep at a pixel dimension of 1 x $2.05 \mu \mathrm{m}$ (lateral-axial).

The used acquisition method, including data export, had a total processing time of roughly 40 seconds per site. More details on the specific acquisition parameters of the OCT can be found in the electronic supporting information.

Post-processing consisted of converting scan data into 32-bit floating point CSV files using the instrument software. Data was imported, normalized and further processed using a custom MATLAB script (MATLAB 2017b using the built-in image processing toolbox). Images were processed by a multi-step sequence: (1) image contrast was improved and equalized by applying contrast-limited adaptive histogram equalisation (CLAHE) using the "adapthisteq" function (Figure 3A); (2) The position of the lower part of the FTO layer (interfacing with biofilm) was determined. Assuming an (almost) perpendicular positioning 


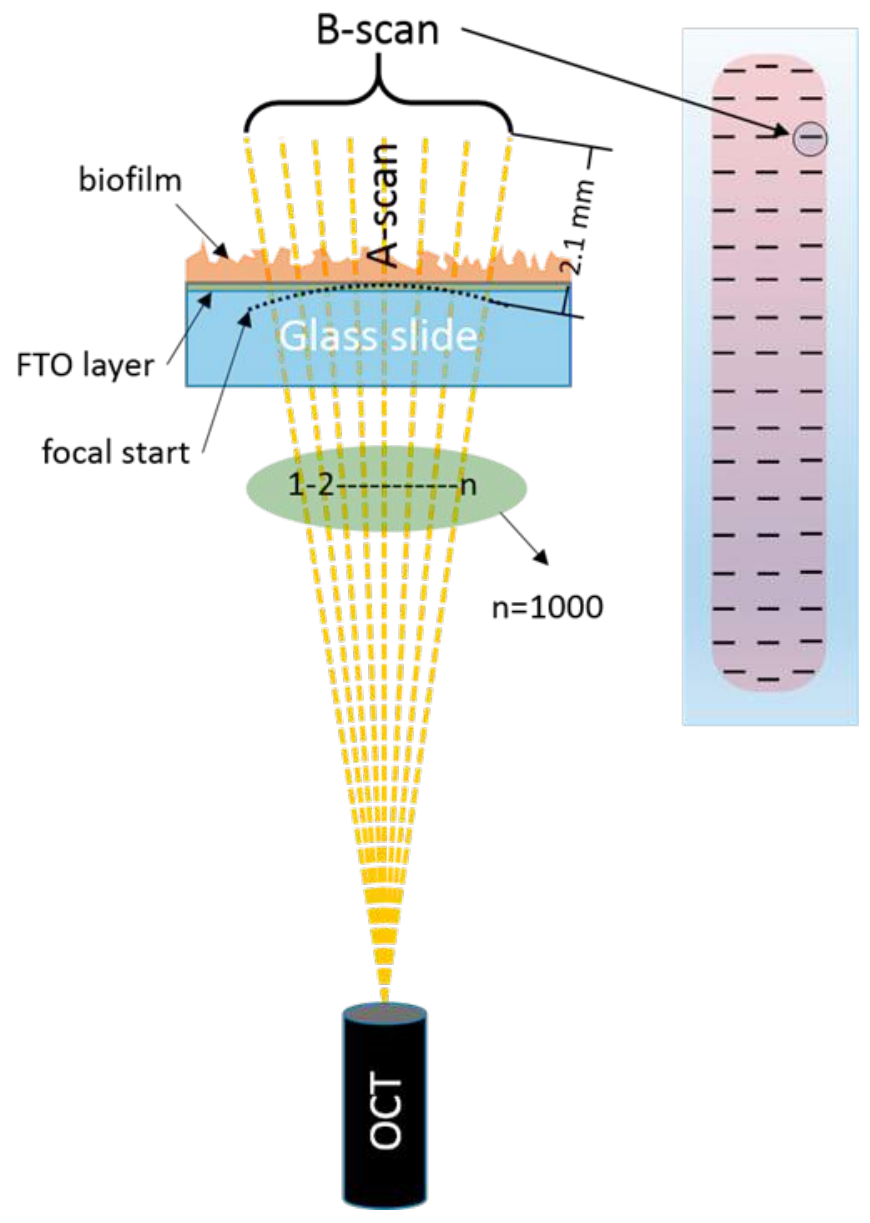

FIGURE 2 | Schematic overview of the acquisition method used for the OCT. A single B-scan was composed of 1000 A-scans. On the right a schematic FTO electrode with biofilm is depicted. The 54 individual B-scans taken from predefined spots during OCT analysis are indicated by black lines.

of FTO layer with respect to the scan direction, a row vector was calculated, containing the mean for each row. Differences between adjacent rows from this row vector were calculated, and the row containing the minimal (most negative) resulting difference was determined.After the determination of this row number, a mask of zeros was applied to the image for all the pixels above that row number, removing the FTO electrode image and everything above, i.e. electrolyte (Figure 3B); (3) To isolate the biomass structure, edge detection (Sobel method) was used. This returned edges at those points where the gradient on the picture was above a set threshold value; (4) Since the edge detection led 


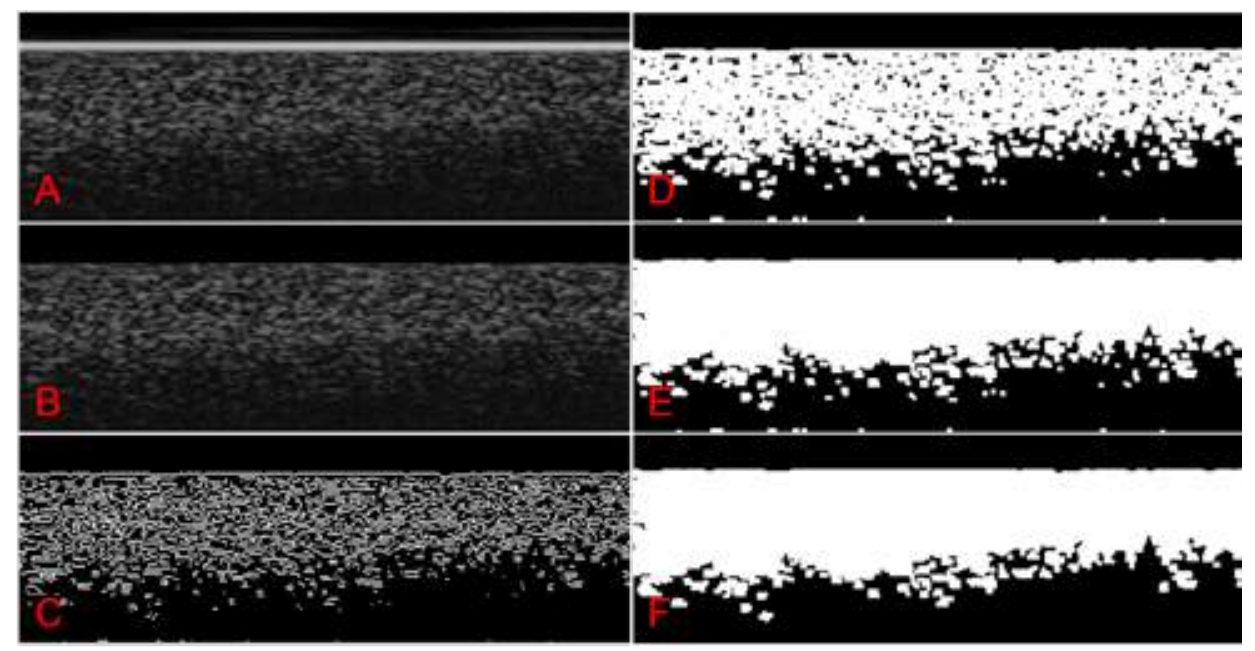

Figure 3 Step-by-step visualisation of the image processing involved with OCT analysis. A) image after adaptive histogram equalisation, clearly depicting the bright FTO layer on top of the image B) FTO layer and all above removed, C) binary image after edge-detection, D) after "gluing" of detected edges, E) after filling holes, F) after removing loose and small particles.

to some noise amplification, two consecutive operations were performed in order to remove this: rows for which the row-sum was higher than a threshold value were set to zero. Then, edges with more than 100 connected pixels were removed. What remained was a binary contour image of (presumably) only the biofilm, containing holes and breaches (Figure 3C); (5) Breaches were closed using the dilate function (imdilate, Figure 3D), after which holes were filled with the built-in function "imfill”. Some noise below the biofilm was still visible at this point (Figure 3E); (6) As last polishing steps, all objects smaller than 30 pixels were removed and pixel value of the last two rows were set to zero. The latter was done to correct for artificial noise created by the edge detection step (Figure 3F); (7) In the obtained binary picture, true pixels (value 1) represented the biomass. The pixel counts for each of the 54 cross-sections analysed per electrode were then averaged and the average biofilm thickness was obtained by dividing the average pixel count by the lateral pixel scaling factor. Biofilm volume was calculated by multiplying the average biofilm thickness with the electrode surface area. The complete MATLAB script is disclosed as electronic supporting information. 


\subsubsection{Chemical analyses}

After dismantling the cell, the biofilm was first gently rinsed with a $100 \mathrm{mM}$ phosphate buffer solution to remove residual soluble organics, chloride and ammonium while preventing acute osmotic stress. The biofilm was then mechanically detached from the glass electrode using a conventional cell scraper. The wet biomass pellet weight was measured and then suspended with the use of vortex and sonication in a defined volume $(10 \mathrm{~mL}$ of $100 \mathrm{mM}$ phosphate buffer solution). The resulting homogenized samples were analysed for chemical oxygen demand (COD) and total nitrogen content (TN), using photometric test kits (HachLange LCK 114 and 138, respectively). Assuming a volumetric density of $1 \mathrm{~g} / \mathrm{mL}$ for wet biomass, the absolute amounts of COD and total $\mathrm{N}$ for each electrode were then calculated.

\subsubsection{Calculations and conversions used to obtain biomass growth yield}

Assuming biomass formation as the only competing process to current generation from acetate oxidation, we can express the electron balance in our systems as:

$$
e_{\text {electrode }}+e_{\text {biomass }}=-e_{\text {substrate }}
$$

In this simple expression, $e_{\text {electrode }}$ represents the amount of electrons channelled to the electrode, thus representing catabolic activity of the present biomass. $e_{\text {biomass }}$ equals the amount of electrons which ends up in the formation of biomass, thus representing anabolic activity.

The electrons expressed as $\boldsymbol{e}_{\text {substrate }}$ are derived from substrate oxidation (acting as electron donor, negative by convention).

The yield coefficient, by convention, expresses the ratio of electrons used for biomass growth (i.e. anabolism) to electrons derived from substrate oxidation [Eq (2)]:

$$
Y=\frac{e_{\text {biomass }}}{-e_{\text {substrate }}}
$$

To determine the biomass growth yield, we use biomass volume from the presented OCT measurements, in combination with the total produced charge (as measured by chronoamperometry). First, the biomass volume as measured by OCT $\left(V_{\text {biomass }}[\mathrm{mL}]\right)$ was converted into $\mathrm{COD}_{\text {biomass }}\left[\mathrm{g}_{\mathrm{COD}}\right]$ by using a linear correlation established in this study (Figure 4B)[Eq (3)]:

$$
C O D_{\text {biomass }}=a V_{\text {biomass }}
$$


With $\alpha$ being the regression coefficient.

The obtained grams of COD biomass were then converted into $\mathrm{e}_{\text {biomass }}[\mathrm{mol}]$ using Equation (4):

$$
e_{\text {biomass }}=\frac{n \text { CoD biomass }}{M_{O_{2}}}
$$

In which $M_{O_{2}}$ is the molecular weight of dioxygen (32 g) and $n$ the electron stoichiometry involved with complete reduction of dioxygen (4).

The accumulated charge (Q) on the electrode (electron acceptor) is expressed in $e$ by division with the Faraday constant (F), [Eq (5)]:

$$
e_{\text {electrode }}=\frac{Q}{F}
$$

Thus, by combining Equations 1-5, Equation 6 is obtained:

$$
Y=\frac{e_{\text {biomass }}}{e_{\text {electrode }}+e_{\text {biomass }}}=\frac{1}{1+\frac{e_{\text {electrode }}}{e_{\text {biomass }}}}=\frac{1}{\left(1+\frac{M_{\mathrm{O}_{2} Q} \mathrm{Q}}{\text { cnnV }_{\text {biomass }}}\right)}
$$

Moreover, as by convention Coulombic efficiency (CE) is defined as the ratio of the amount of electrons channelled to the acceptor $\left(\mathrm{e}_{\text {electrode }}\right)$ to the amount of electrons derived from substrate oxidation $\left(\mathrm{e}_{\text {substrate }}\right)$, by using equation 5 , the following definition for $\mathrm{CE}$ is obtained [Eq (7)]:

$$
C E=\frac{e_{\text {electrode }}}{-e_{\text {substrate }}}=1-Y
$$

It should be noted here however, in case CE is defined on basis of biomass production measurements, this can only reflect a theoretical maximally attainable CE. Actual CEs may be lower if unreported side-processes not leading to detected biomass growth are also present.

\subsection{RESULTS AND DISCUSSION}

\subsubsection{OCT analysis allows for accurate and precise in situ bio- mass quantification}

For all runs, starting with a clean electrode, initially small negative currents $\left(-0.04\right.$ to $\left.0 \mathrm{~A} / \mathrm{m}^{2}\right)$ were observed, before a positive current developed. This initial phase in which practically no current was generated, was defined as lag-phase, and varied typically from one to three days amongst runs. To allow for a better comparison of current development between individual runs, Figure 4A shows the current development after these lag-phases were finished, thus letting each 
curve start once currents became positive. The different experimental durations which are reflected by shorter or longer curves are also visible in Figure 4A. As a main trend, current initially increased exponentially, peaking at around 1 to $2 \mathrm{~A} / \mathrm{m}^{2}$, after which a gradual decrease followed, eventually stabilising at current densities of approximately $1 \mathrm{~A} / \mathrm{m}^{2}$. The obtained current densities during the studies' term are comparable to flat plate bioanodes previously reported at similar anode potential ${ }^{[58,59]}$. This legitimates the extrapolation of results obtained with FTO as electrode material towards more conventional electrode materials and geometries. On a longer timescale, reduced electrode conductivity occurred in some of the used FTO electrodes, with three runs showing considerably less current production over time as a result. After dismantling these systems, the reduced current production turned out to be caused by parts of the FTO layer being damaged, with only parts of the electrode colonized. The mechanism causing this damage was not further investigated. Nevertheless, as the presented method should also be applicable and robust in situations of nonhomogeneous biofilm coverage, the results of these three runs were included in further analysis.

Obtained biofilm thicknesses ranged between 3 to $90 \mu \mathrm{m}$ amongst all measured samples. Biomass volume as measured by OCT was calculated by multiplying the average biofilm thickness with the effective electrode area (22.3 $\mathrm{cm}^{2}$ ). Figure 4B and $\mathbf{C}$ compare the obtained measurement biofilm volumes to total biofilm COD and total nitrogen, respectively. Both COD and total $\mathrm{N}$ show a strong linear relation with biofilm volume as measured by OCT. With coefficients of determination well above 0.95 , OCT showed itself a reliable estimator for both biofilm COD and $\mathrm{N}$ content in these experiments. From these data, COD/N ratio was determined to be 18.7:1 by linear regression $\left(\mathrm{R}^{2}=0.97\right)$. Assuming a conversion ratio between COD and $\mathrm{TOC}^{[60]}$ of $3: 1$, this converts to a C:N ratio in the measured biomass of approximately $6: 1$, which is within the range of previously reported C: $\mathrm{N}$ ratios for microbial biomass ${ }^{[61]}$. As a side note regarding the linearity between OCT and COD/total N, a tendency of OCT analysis slightly overestimating biofilm volume in the lower volume region $(0-0.02 \mathrm{~mL})$ was observed. For these measurements in which only a very thin biofilm was present, a slightly increased but inevitable noise amplification occurred due to (i) increased OCT autocorrelation artefacts associated with a clean FTO/electrolyte interface and (ii) increased noise amplification by the adaptive histogram equalisation. These observations thus limit the precision of the presented method in this starting domain of early biofilm attachment and growth, but validate its use over a broader range of biofilm thicknesses.

To assess the accuracy of OCT for measuring absolute volumetric quantities of biomass, in a fourth figure (Figure 4D) the biomass volume as measured by OCT is plotted against the biomass wet pellet weight. Assuming a specific density 

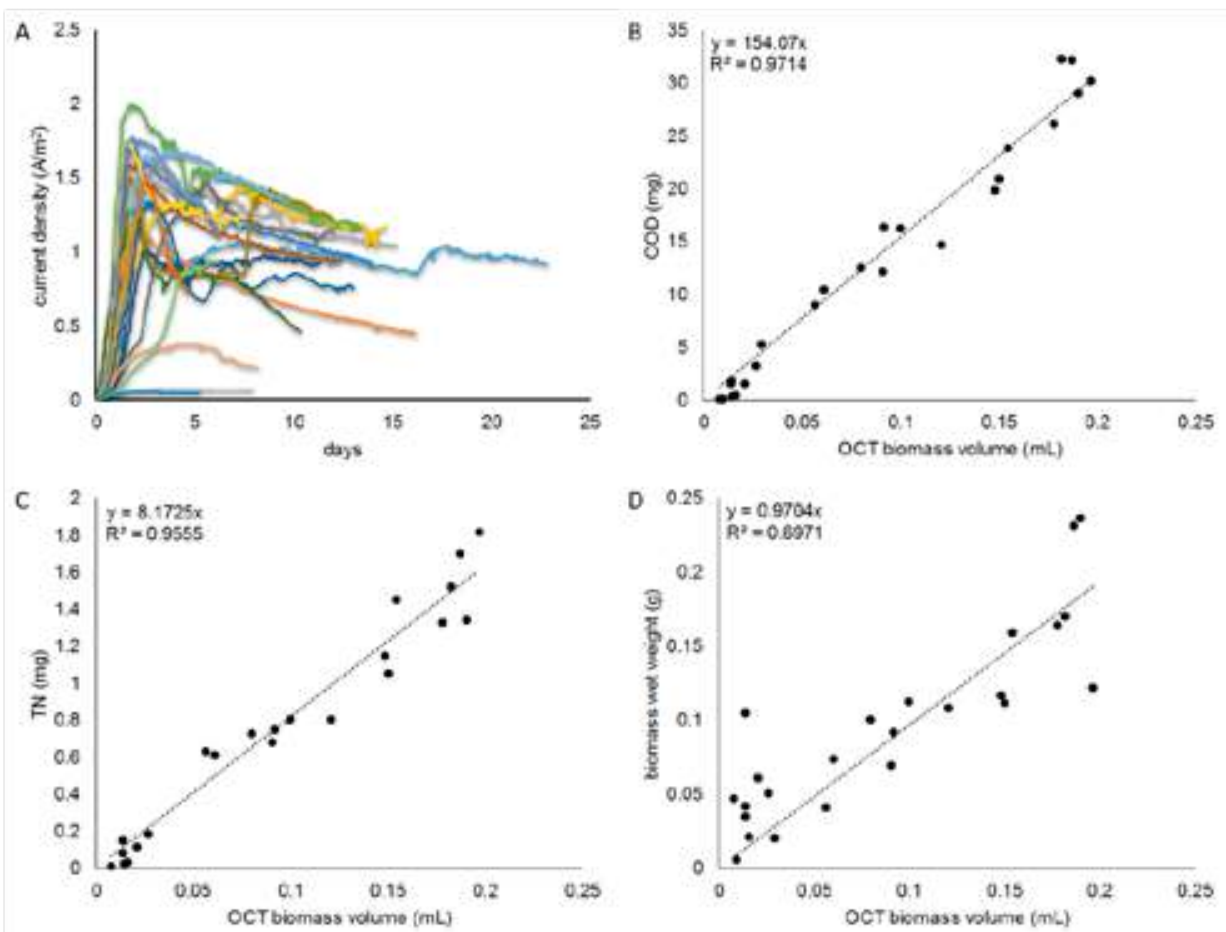

FIGURE 4 | (A) Current density development for the 24 individual runs, B,C and D) biomass COD, total N, and wet weight plotted versus the biomass volume as measured by OCT. COD and total N were normalized to the total FTO surface area, thus provide an absolute measure.

of wet biomass similar to that of water $(1 \mathrm{~g} / \mathrm{L})$, a 1-to-1 linear relation would be expected between these two variables. The linear regression for these two variables indeed showed a slope of nearly 1, supporting used method for measuring biomass volume in terms of its accuracy. The lower $\mathrm{R}^{2}$ value for this fit somewhat weakens this position, but it is to our best understanding the inevitable imprecisions and inaccuracies involved with weighing of small amounts of wet biomass - thus not the OCT methods applied - which created considerable additional variance, leading to this lower $\mathrm{R}^{2}$.

\subsubsection{Determination of biomass growth yield using a combina- tion of OCT and chronoamperometry}

Apart from biomass growth rates, the biomass growth yield, or yield ratio, is a parameter of importance in most BES studies. Biomass yield ratio defines to a great extent the energy efficiency and thus system performance, and is commonly 
expressed as gram COD biomass gained per gram of COD substrate consumed, with one mole of COD being equivalent to four mole of electrons. Accordingly, the yield ratio represents the amount of electrons used for anabolism (creation of biomass) compared to the total amount of electrons derived from oxidizing the electron donor, including catabolism (substrate turnover for energy production required for growth).

Specifically for electrogenic biomass, the substrate consumed for catabolic processes is directly linked to current production. With biomass volume being linearly related to biomass COD (Figure 4B), biomass growth yield can be assessed indirectly by plotting biofilm volume versus total charge produced during biofilm growth, and this is done in Figure 5.

We observed a good match between biofilm volume as measured by OCT and total charge: with more charge produced, biofilm volume increased. As can be inferred from this linearity, yields throughout the different runs were rather constant. This would be in line with growth conditions (and most notably anode potential) for each experiment being the same, and the biofilm being harvested being still in growth stage (thus with none of the grown biomass being washed out or detached from the electrode, and no substantial changes or impact of maintenance on overall growth yields).

Elaborating on this assumption of linearity and constant growth yields, and in order to avoid error propagation in calculating yields from individual measurements, the overall yield of biomass on current was taken as the slope of the regression line: $1 \mathrm{~mL}$ biomass / $40705 \mathrm{C}$ electrons. This value for $\mathrm{V}_{\text {biomass }}: \mathrm{Q}$ was then inserted into equations 6-7 to translate into $42.15 \mathrm{mg} \mathrm{COD}$ biomass $/ \mathrm{g} \mathrm{COD}_{\text {acetate }}$ $(\mathrm{CE}=0.958)$. This obtained yield is a feasible value, being within range of earlier

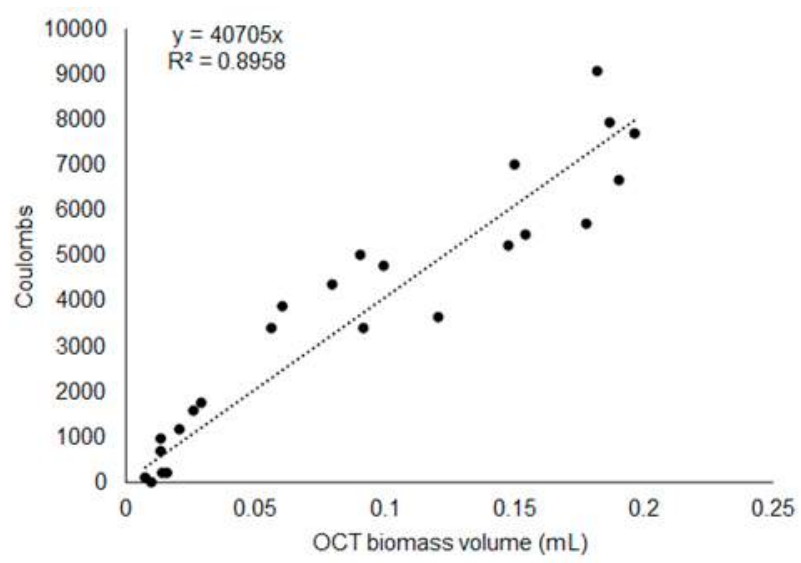

FIGURE 5 | Scatter and regression slope of the measured biomass volume by OCT against the total amount of charge (Coulombs) obtained from the biofilm 
reported biomass yields ${ }^{[27]}$ and lower but comparable to the theoretical maximum biomass yield of $109 \mathrm{mg} \mathrm{COD}$ biomass $/ \mathrm{g} \mathrm{COD}_{\text {acetate }}(\mathrm{CE}=0.891)$ calculated at the used anode potential of $-0.35 \mathrm{~V}$ using the thermodynamic approach from Picioreanu et $\mathrm{al}^{[32]}$. Thus, we show that using a combination of OCT and chronoamperometric data - both non-invasive measurements - we can derive valid biomass growth yields from the tested systems.

\subsubsection{Continuous measurement confirms application of OCT for non-invasive assessment of biofilm growth and activity}

Two independent bioanodes were grown to confirm the applicability of OCT for tracking biofilm growth over time. Figure 6 shows cumulative charge and OCT derived estimates for biofilm volume obtained from these two cells for which timelapse observations on biofilm growth were conducted. Figure 6A shows results for cell 1, which was operated for a total of 14 days, and Figure 6B shows results for the second cell, operated for a period of 17 days. From the measurements of biofilm volume and accompanying charge consumption, biomass yield estimates were obtained by linear regression. These are visualized for both cells in Figure 7, and the high R2 obtained strongly suggests a constant yield throughout the experimental period for both cells. The obtained yield estimates from these regressions for both cells were significantly similar to each other (38.7 and 38.8 $\mathrm{mg} \mathrm{COD}$ biomass $/ \mathrm{COD}_{\text {acetate }}$, $\mathrm{p}=0.976$ under the null hypothesis of identical slopes), thus showing high reproducibility of the used methods.

In general, current production resumed fast and completely after OCT measurements were performed and systems were electronically and hydraulically reconnected. However, upon reconnecting on day 9, inaccurate potential control for cell 2 led to anode potential fluctuating between -0.35 and $-0.45 \mathrm{~V}$ throughout

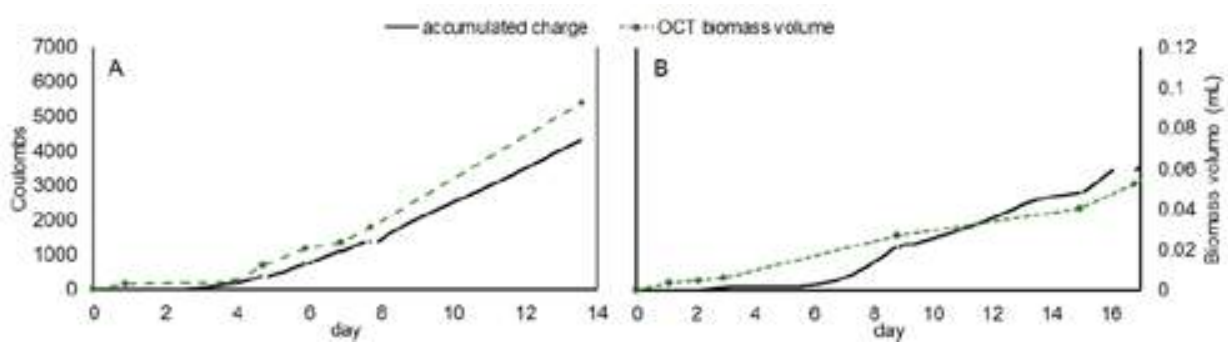

FIGURE 6 | Development of charge (black) and biofilm volume (as measured by OCT, green, dashed, with markers) during two independent continuous experiments. In these experiments, cells were resumed to produce current after each OCT measurement. A) depicts results for a cell run for a total of 14 days, B) a cell in which the last OCT measurement was conducted at 17 days since start. 


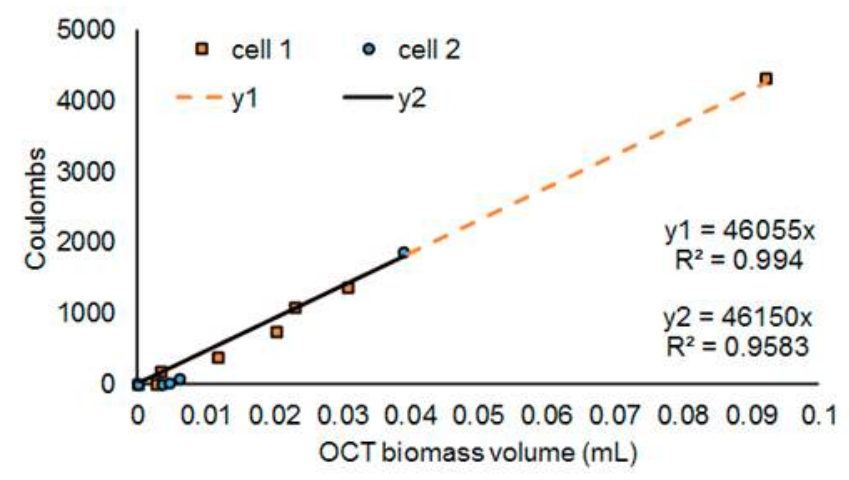

Figure 7 Relation between accumulated charge and biomass volume as measured by OcT, providing a measure of yield, for both time-lapse operated cells. In above representation, the data for cell 2 for the periods in which cell conditions were unknown or fluctuating was removed. The straight lines (y1 in dashed orange for cell 1, y2 in solid black for cell 2) are obtained by linear regression, and the slopes resulting from these fits and their $\mathrm{R}^{2}$ values are included. The high correlation coefficients obtained, suggest strongly constant yields throughout the experimental period. Yields were calculated from these obtained slopes to be 38.7 and $38.8 \mathrm{mg}$ CODbiomass / g CODacetate for cell 1 and 2, respectively, and an additional T-test showed them to be significantly the same $(p=0.976)$.

the period of day 9 to 15 . Over this period of fluctuating potential, an incremental yield estimate of $16 \mathrm{mg} \mathrm{COD}$ biomass $/ \mathrm{gCD}_{\text {acetate }}$ (CE $=0.984$ ) was obtained, roughly half as large as in the case of yields obtained when potential was controlled steadily at $-0.35 \mathrm{~V}$. Although unintended, this suggests fast adaptive behaviour of the present biofilm to potential change. Due to this artefact, this period was left out of the analysis as depicted in Figure 7.

Overall, the slightly lower yields obtained with the time-lapse experiments as compared to the sacrificial calibration experiments, may be explained by the presence of periods during which cells were not connected to the potentiostat, leading to a slightly lower overall anode potential for the continuous runs.

\subsection{CONCLUSIONS}

In conclusion, we have demonstrated here that accurate and precise measurements of biomass volume can be obtained in-situ and non-invasively from active bioanodes by using OCT in BESs. Doing so, this allows for biomass yield and CE estimates to be obtained at high statistical precision and real-time. The method developed showed OCT results to be linearly correlated to conventional biomass quantifications by COD and total $\mathrm{N}$. As such, the presented method allows for a 
more complete and meaningful characterisation of BESs, especially when used in combination with additional in-situ measurements like $\mathrm{pH}$, off-gas analysis and/or electrolyte chemical analysis. When such characterizations are performed at different experimental conditions, this will provide valuable data for a deeper understanding of mechanisms at play in BESs, especially in the field of bioenergetics. It is anticipated to lead to identification of deviations from assumed thermodynamic model assumptions, or rate limiting factors/processes.

As a direct illustration of this, previous publications have proposed metabolic activity inside electroactive biofilms to be potentially limited by both diffusion and conductivity ${ }^{[62,63]}$. Which phenomena actually limit current production may be very specific to reactor design and operational parameters. One could imagine a limiting current density could also have an effect on the microbial growth yield, as the biofilm grows thicker. However, our data shows constant growth yields throughout experimental runs while experimental duration exceeded (by far) the moment of maximum current production, thus with current being limited, and to various extents (Figure 4A). Although not incompatible with the mechanistic models on biofilm growth and activity postulated previously, the observation of constant growth yields in our current experiments raises new questions regarding how biofilm dynamics, and current limiting factors as they emerge, affect system efficiency in BESs. A combined approach, which integrates OCT for tracking biofilm growth, may show advantageous in finding answers.

The used FTO electrodes enable this concomitant application of different electrochemical and visualisation techniques, e.g. confocal Raman spectroscopy, confocal laser scanning microscopy, and more sophisticated electrochemical methods like cyclic voltammetry and electrochemical impedance spectroscopy. These electrodes therefore provide ample opportunities for studying in-depth the kinetic properties and mechanisms of electron transport in BESs, as well as allowing for high-resolution visualisation and functionalized imaging of living biofilms.

\subsection{ACKNOWLEDGEMENTS}

This work was performed in the cooperation framework of Wetsus, European Centre of Excellence for Sustainable Water Technology (www.wetsus.eu). Wetsus is co-funded by the Dutch Ministry of Economic Affairs and Ministry of Infrastructure and Environment, the Province of Fryslân, and the Northern Netherlands Provinces. The authors like to thank the participants of the Resource Recovery research theme for the fruitful discussions and their financial support. 


\subsection{REFERENCES}

[1] D. R. Lovley, Energy Environ. Sci. 2011, 4, 4896.

[2] G. Reguera, K. D. McCarthy, T. Mehta, J. S. Nicoll, M. T. Tuominen, D. R. Lovley, Nature 2005, 435, 1098-1101.

[3] D. R. Lovley, Annu. Rev. Microbiol. 2012, 66, 391-409.

[4] S. D. Molenaar, A. R. Mol, T. H. J. A. Sleutels, A. ter Heijne, C. J. N. Buisman, Environ. Sci. Technol. Lett. 2016, DOI 10.1021/acs.estlett.6b00051.

[5] A. Deeke, T. H. J. A. Sleutels, H. V. M. Hamelers, C. J. N. Buisman, Environ. Sci. Technol. 2012, 46, 35543560 .

[6] B. E. Logan, B. Hamelers, R. Rozendal, U. Schröder, J. Keller, S. Freguia, P. Aelterman, W. Verstraete, K. Rabaey, Environ. Sci. Technol. 2006, 40, 5181-5192.

[7] B. E. Logan, D. Call, S. Cheng, H. V. M. Hamelers, T. H. J. A. Sleutels, A. W. Jeremiasse, R. A. Rozendal, Environ. Sci. Technol. 2008, 42, 8630-8640.

[8] P. Ledezma, P. Kuntke, C. J. N. Buisman, J. Keller, S. Freguia, Trends Biotechnol. 2015, 33, 214-220.

[9] K. Rabaey, R. A. Rozendal, Nat. Rev. Microbiol. 2010, 8, 706-716.

[10] B. E. Logan, K. Rabaey, Science (80-. ). 2012, 337, 686-690.

[11] C. Donovan, A. Dewan, D. Heo, H. Beyenal, Environ. Sci. Technol. 2008, 42, 8591-8596.

[12] D. P. B. T. B. Strik, R. A. Timmers, M. Helder, K. J. J. Steinbusch, H. V. M. Hamelers, C. J. N. Buisman, Trends Biotechnol. 2011, 29, 41-49.

[13] N. E. Stein, K. J. Keesman, H. V. M. Hamelers, G. van Straten, Biosens. Bioelectron. 2011, 26, 3115-3120.

[14] L. Su, W. Jia, C. Hou, Y. Lei, Biosens. Bioelectron. 2011, 26, 1788-1799.

[15] N. S. Malvankar, T. Mester, M. T. Tuominen, D. R. Lovley, ChemPhysChem 2012, 13, 463-468.

[16] Y. Liu, D. R. Bond, ChemSusChem 2012, 5, 1047-1053.

[17] S. Freguia, K. Rabaey, Z. Yuan, J. Keller, Environ. Sci. Technol. 2007, 41, 2915-2921.

[18] D. R. Lovley, Curr. Opin. Biotechnol. 2008, 19, 564-571.

[19] E. Marsili, D. B. Baron, I. D. Shikhare, D. Coursolle, J. A. Gralnick, D. R. Bond, Proc. Natl. Acad. Sci. 2008, 105, 3968-3973.

[20] C. I. Torres, A. K. Marcus, B. E. Rittmann, Biotechnol. Bioeng. 2008, 100, 872-881.

[21] F. Kracke, I. Vassilev, J. O. Kr??mer, Front. Microbiol. 2015, 6, 1-18.

[22] A. ter Heijne, O. Schaetzle, S. Gimenez, L. Navarro, B. Hamelers, F. Fabregat-Santiago, Bioelectrochemistry 2015, 106, 64-72.

[23] W. Zhi, Z. Ge, Z. He, H. Zhang, Bioresour. Technol. 2014, 171, 461-468.

[24] B. E. Logan, ChemSusChem 2012, 5, 988-994.

[25] R. A. Rozendal, H. V. M. Hamelers, K. Rabaey, J. Keller, C. J. N. Buisman, Trends Biotechnol. 2008, 26, 450-459.

[26] T. H. J. A. Sleutels, A. Ter Heijne, C. J. N. Buisman, H. V. M. Hamelers, ChemSusChem 2012, 5, $1012-1019$.

[27] P. Aelterman, S. Freguia, J. Keller, W. Verstraete, K. Rabaey, Appl. Microbiol. Biotechnol. 2008, 78, 409418.

[28] J. Wei, P. Liang, X. Cao, X. Huang, Environ. Sci. Technol. 2010, 44, 3187-3191.

[29] M. D. Yates, P. D. Kiely, D. F. Call, H. Rismani-Yazdi, K. Bibby, J. Peccia, J. M. Regan, B. E. Logan, ISME J. 2012, 6, 2002-2013. 
[30] S. T. Read, P. Dutta, P. L. Bond, J. Keller, K. Rabaey, BMC Microbiol. 2010, 10, 98.

[31] T. H. J. A. Sleutels, L. Darus, H. V. M. Hamelers, C. J. N. Buisman, Bioresour. Technol. 2011, DOI 10.1016/j. biortech.2011.09.078.

[32] C. Picioreanu, I. M. Head, K. P. Katuri, M. C. M. van Loosdrecht, K. Scott, Water Res. 2007, 41, $2921-2940$.

[33] H. V. M. Hamelers, A. ter Heijne, N. Stein, R. A. Rozendal, C. J. N. Buisman, Bioresour. Technol. 2011, 102, 381-387.

[34] J. Rodríguez, J. M. Lema, R. Kleerebezem, Trends Biotechnol. 2008, 26, 366-374.

[35] A. E. Franks, N. Malvankar, K. P. Nevin, Biofuels 2010, 1, 589-604.

[36] M. R. Khan, E. Baranitharan, D. M. R. Prasad, C. K. Cheng, MATEC Web Conf. 2016, 62, 4002.

[37] L. Jourdin, T. Grieger, J. Monetti, V. Flexer, S. Freguia, Y. Lu, J. Chen, M. Romano, G. G. Wallace, J. Keller, Environ. Sci. Technol. 2015, 49, 13566-13574.

[38] L. Chen, P.-L. Tremblay, S. Mohanty, K. Xu, T. Zhang, J. Mater. Chem. A 2016, 4, 8395-8401.

[39] K. P. Katuri, A. M. Enright, V. O'Flaherty, D. Leech, Bioelectrochemistry 2012, 87, 164-171.

[40] S. D. Molenaar, P. Saha, A. R. Mol, T. H. J. A. Sleutels, A. ter Heijne, C. J. N. Buisman, Int. J. Mol. Sci. 2017, $18,204$.

[41] A. E. Franks, K. P. Nevin, H. Jia, M. Izallalen, T. L. Woodard, D. R. Lovley, Energy Environ. Sci. 2009, 2, 113-119.

[42] G. T. Kim, G. Webster, J. W. T. Wimpenny, B. H. Kim, H. J. Kim, A. J. Weightman, J. Appl. Microbiol. 2006 , 101, 698-710.

[43] B. H. Kim, H. S. Park, H. J. Kim, G. T. Kim, I. S. Chang, J. Lee, N. T. Phung, Appl. Microbiol. Biotechnol. 2004, 63, 672-681.

[44] J. R. Lawrence, T. R. Neu, Methods Enzymol. 1999, 310, 131-144.

[45] T. R. Neu, B. Manz, F. Volke, J. J. Dynes, A. P. Hitchcock, J. R. Lawrence, FEMS Microbiol. Ecol. 2010, 72, $1-21$.

[46] N. Kamjunke, U. Spohn, M. Füting, G. Wagner, E. M. Scharf, S. Sandrock, B. Zippel, Int. Biodeterior. Biodegrad. 2012, 69, 17-22.

[47] J. M. Vroom, K. J. De Grauw, H. C. Gerritsen, D. J. Bradshaw, P. D. Marsh, G. K. Watson, J. Birmingham, C. Allison, K. J. D. E. Grauw, 1999, 65, 3502-3511.

[48] J. P. Busalmen, S. R. De Sánchez, Biotechnol. Bioeng. 2003, 82, 619-624.

[49] X. Jiang, J. Hu, E. R. Petersen, L. A. Fitzgerald, C. S. Jackan, A. M. Lieber, B. R. Ringeisen, C. M. Lieber, J. C. Biffinger, Nat. Commun. 2013, 4, DOI 10.1038/ncomms3751.

[50] J. S. McLean, G. Wanger, Y. A. Gorby, M. Wainstein, J. McQuaid, Env. Sci. Technol. 2010, 44, 2721-2717.

[51] S. B. Surman, J. T. Walker, D. T. Goddard, L. H. G. Morton, C. W. Keevil, W. Weaver, A. Skinner, K. Hanson, D. Caldwell, J. Kurtz, J. Microbiol. Methods 1996, 25, 57-70.

[52] V. Reipa, J. Almeida, K. D. Cole, J Microbiol Meth 2006, 66, 449-459.

[53] Y. Ding, Y. Zhou, J. Yao, C. Szymanski, J. Fredrickson, L. Shi, B. Cao, Z. Zhu, X.-Y. Yu, Anal. Chem. 2016, 88, 11244-11252.

[54] B. Virdis, F. Harnisch, D. J. Batstone, K. Rabaey, B. C. Donose, Energy Environ. Sci. 2012, 5, 7017.

[55] D. Huang, E. A. Swanson, C. P. Lin, J. S. Schuman, W. G. Stinson, W. Chang, M. R. Hee, T. Flotte, K. Gregory, C. A. Puliafito, et al., Science (80-. ). 1991, 254, 1178-1181.

[56] C. Dreszer, A. D. Wexler, S. Drusová, T. Overdijk, A. Zwijnenburg, H. C. Flemming, J. C. Kruithof, J. S. Vrouwenvelder, Water Res. 2014, 67, 243-254. 
[57] J. G. Fujimoto, J. M. Schmitt, E. A. Swanson, I.-K. Jang, in (Ed.: I.-K. Jang), Springer International Publishing, Cham, 2015, pp. 1-21.

[58] A. ter Heijne, H. V. M. Hamelers, M. Saakes, C. J. N. Buisman, Electrochim. Acta 2008, 53, 5697-5703.

[59] A. Dekker, A. Ter Heijne, M. Saakes, H. V. M. Hamelers, C. J. N. Buisman, Environ. Sci. Technol. 2009, 43, 9038-9042.

[60] D. Dubber, N. F. Gray, J. Environ. Sci. Heal. - Part A Toxic/Hazardous Subst. Environ. Eng. 2010, 45, 15951600.

[61] S. V. Vassilev, D. Baxter, L. K. Andersen, C. G. Vassileva, Fuel 2010, 89, 913-933.

[62] D. R. Bond, S. M. Strycharz-Glaven, L. M. Tender, C. I. Torres, ChemSusChem 2012, 5, 1099-1105.

[63] D. Sun, S. Cheng, A. Wang, F. Li, B. E. Logan, K. Cen, Environ. Sci. Technol. 2015, 49, 5227-5235.

[64] Deutsche Sammlung von Mikroorganismen und Zellkulturen, "141. Methanogenium Medium (H2/ CO2)," 2018. 


\subsection{APPENDICES}

\subsubsection{OCT acquisition settings}

The OCT engine was configured at $30 \mathrm{kHz}$ A-scan rate, and initial data processing included A-scan averaging (10 times), B-scan averaging (10 times), this to improve the Signal-to-Noise ratio. An undersampling filter, image field correction, quadratic dispersion compensation (factor 17) and apodisation using the Gauss window, were applied, all of which was included in the instrument software (Thorimage OCT 4.3).

\subsubsection{Matlab script for OCT image processing}

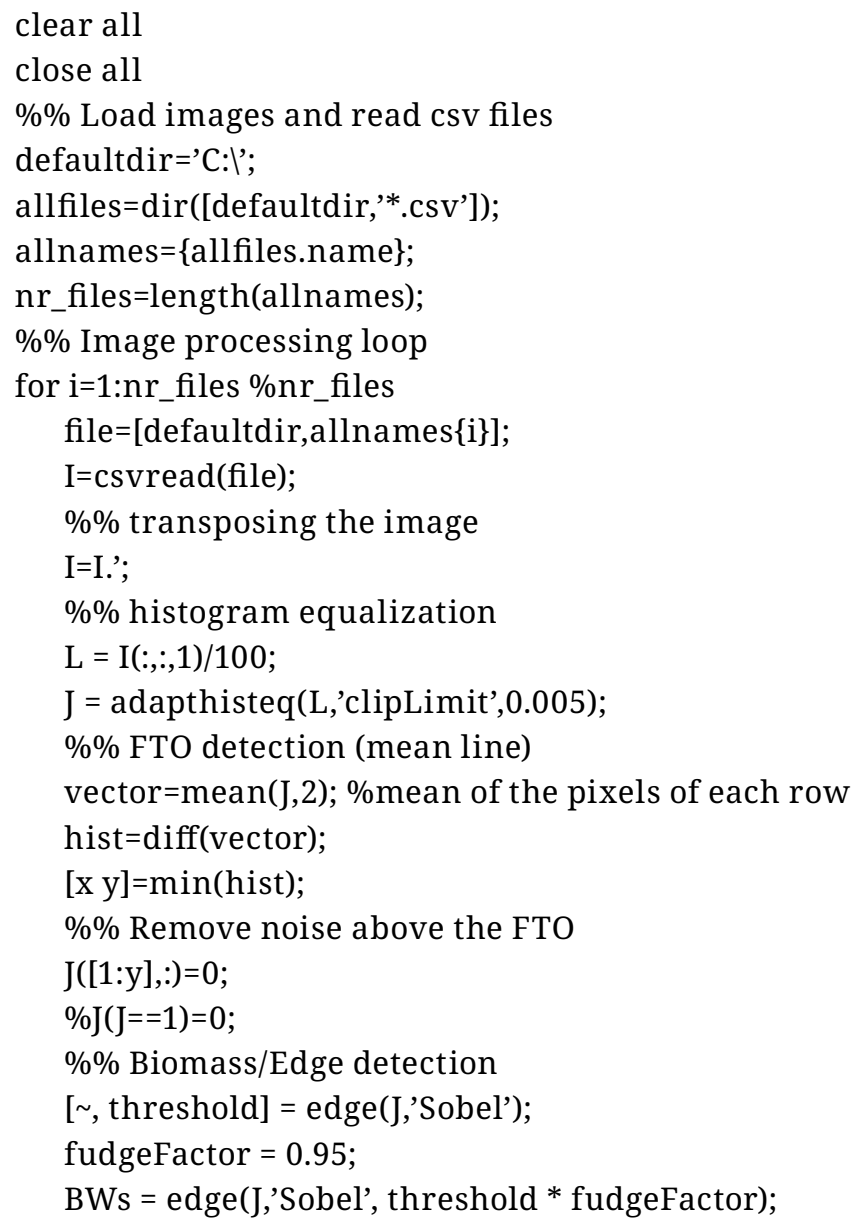


noisepixels=sum(BWs,2);

row=find(noisepixels $>=360$ );

BWs(row,:)=0;

BW2=bwareaopen(BWs,100);

biomassedges=BWs-BW2;

se90 = strel('line', 3, 90);

se0 = strel('line', 9, 0);

BWsdil = imdilate(biomassedges, [se90 se0]);

BWdfill = imfill(BWsdil, 'holes');

$\% \%$ Smoothing

I_s=BWdfill;

\% I_s(I_s>0)=1;

I_s = bwareaopen(I_s,50);

I_s([127:129],:)=0;

biofilm_mass(i)=sum(sum(I_s));

$\% \%$ counting pixels (= biomass quantity) and saving values in a csv file

result_file='C:|results.txt';

fileID = fopen(result_file,'a');

fprintf(fileID,'\%s \%s \%s \%d $\backslash \mathrm{r} \backslash \mathrm{n}^{\prime}$,allnames $\{\mathrm{i}\}(1: 5)$,allnames $\{\mathrm{i}\}$

(15:17),allnames $\{\mathrm{i}\}(19:$ end-4),biofilm_mass(i));

fclose(fileID);

end 


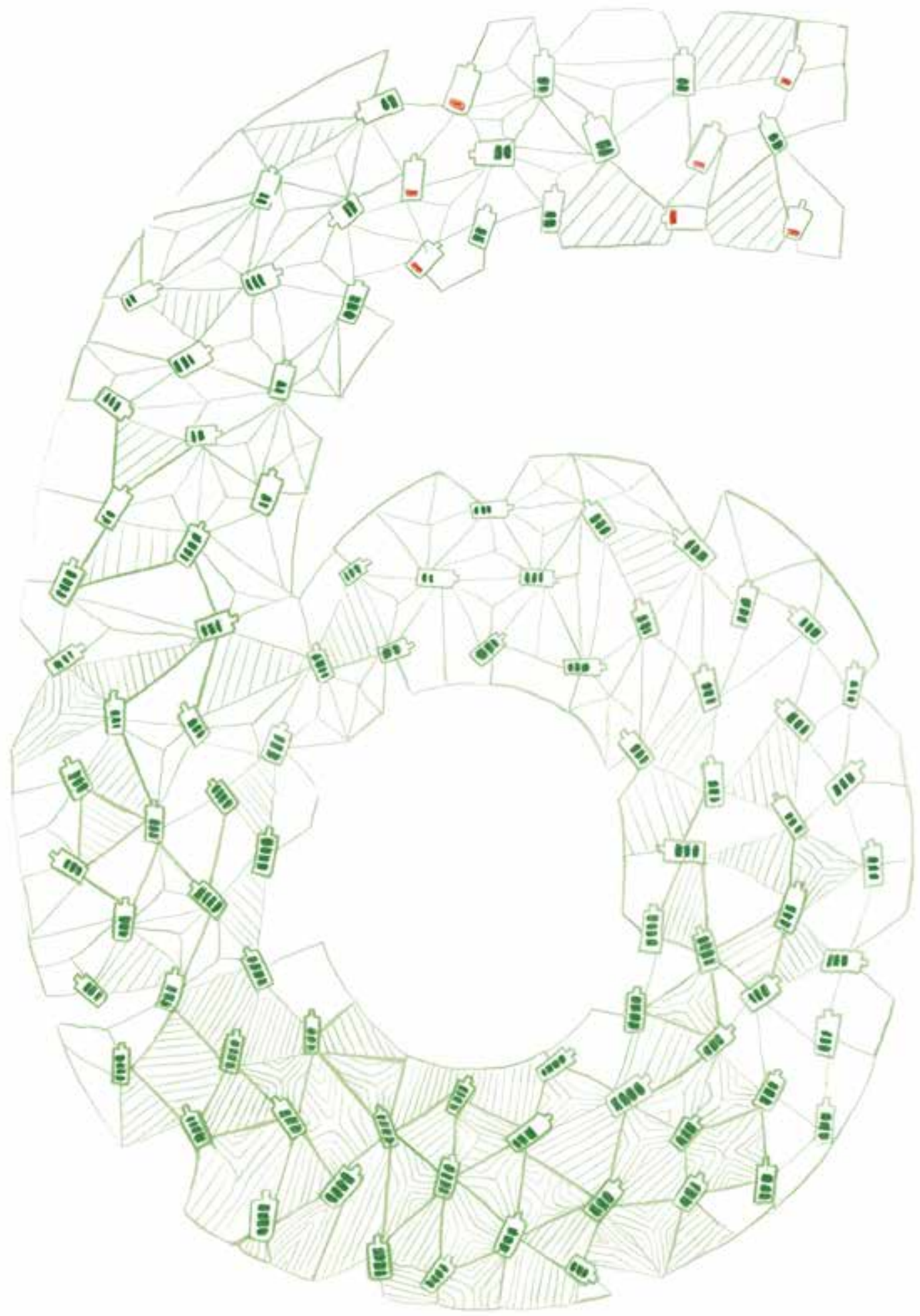




\section{COMPARISON OF TWO SUSTAINABLE COUNTER ELECTRODES FOR ENERGY STORAGE IN THE MICROBIAL RECHARGEABLE BATTERY}

Previously, the microbial rechargeable battery (MRB) has been proposed as a potentially sustainable and low-cost electrical energy storage technology. In the $\mathrm{MRB}$, bioelectrochemical $\mathrm{CO}_{2}$ reduction and subsequent product oxidation has successfully been combined in one integrated system. However, finding a suitable counter electrode is hindering its further development. In this work, we have tested two alternative counter electrodes in duplicate - namely (i) oxygen/water and (ii) a capacitive electrode - for use in the MRB platform. During daily charge/discharge cycling over periods of 11 to 15 days, experimentally obtained energy efficiencies of 25 and $3.7 \%$ were reported when using the capacitive and the oxygen/water electrodes, respectively. Large overpotentials, resulting in a voltage efficiency of $15 \%$ and oxygen crossover leading to Coulombic efficiencies of $25 \%$ caused the considerably lower efficiency for the oxygen/water systems, despite the theoretical higher voltage efficiency. Although the capacitive electrode equipped systems performed better, energy density is limited by the operational potential window within which capacitive systems can operate reliably. Based on the findings presented here, recommendations are given regarding possible future developments in the MRB. 


\subsection{INTRODUCTION}

Wind and solar power represent two major alternative energy sources, a possible transition towards renewable energy could be based on ${ }^{1}$. However, wind and sun are intermittent by nature, and therefore an increasing mismatch between supply and demand of (electrical) energy is foreseen if these renewable sources are to make up for a larger share of total energy supply. One solution to this mismatch is increasing the use of energy storage technologies. Such technologies should comply with the ecological requirements of the current sustainability endeavors undertaken globally ${ }^{2}$.

Recently, we demonstrated the use of the microbial rechargeable battery (MRB) as a potential sustainable energy storage technology ${ }^{3}$. The MRB stores electrical energy as chemical energy through the reduction of carbon dioxide to organic metabolites at a biocathode in a process called microbial electrosynthesis (MES) ${ }^{4-6}$. During discharge, this chemical energy is converted back into electricity through oxidation of the formed metabolites at a bioanode, while the system functions as a microbial fuel cell (MFC) ${ }^{7,8}$.

In order for the MRB to work, the bioanode and biocathode need to be coupled to a counter electrode. This counter electrode needs to possess a sufficiently high potential and reversibility in order to provide substantial cell voltages at useful current densities ${ }^{9}$. In the previously published proof of concept, the ferro/ ferricyanide redox couple was used at the counter electrode, as it is known to be highly active and well soluble, operating at an acceptable potential and with low overpotential under the conditions used, without (expensive) catalysts ${ }^{3}$. Integrating this counter electrode into the MRB resulted in overall energy efficiencies of 30 to $40 \%$ at an utilised energy density of $\sim 100 \mathrm{Wh} / \mathrm{m}^{3}$ and discharge power densities of around $190 \mathrm{~W} / \mathrm{m}^{3}$. The disadvantage of ferro/ferricyanide at the counter electrode is the tendency of the redox couple to form colloidal structures similar or identical to Prussian blue during cycling at the electrode surface, especially in presence of free ferrous iron-ions and at decreased electrolyte mixing, which can impede charge transfer and lead to excessive voltage $\operatorname{losses}^{10}$. Therefore, implementation of ferro/ferricyanide into the MRB offers no long-term sustainable solution and alternative counter electrodes are needed.

This manuscript explores the use of two alternative counter electrodes for the MRB; (i) a capacitive electrode and (ii) an electrode performing the oxygen/water redox reactions, both of which will be shortly discussed.

Storage or withdrawal of charge (by means of an electrical current) in a capacitive electrode takes place exclusively at or from the electrode-electrolyte interface. At this interface, a change in charge density is accompanied by a change in electrostatic double layer (EDL) polarization. As a result of EDL polarization, a linear relation is obtained between electrode potential and (accumulated) charge, 
provided ideal capacitive behavior in which no heat is dissipated ${ }^{11}$. The slope of this linear relation, which depends on electrode material, electrolyte composition and physical conditions, represents the capacitance (in Farad, or Coulomb per Volt). As capacitance is an interface property, it scales up (again, linearly) with the surface area of this interface. Thus, a highly porous electrode material, featuring a high specific surface area, generally has a high capacitance ${ }^{11}$. Capacitive electrode materials are specifically designed to feature high specific surface area with a favorable geometry that minimizes mass transfer losses during polarization at higher current densities. The most important advantage of a capacitive electrode - when used properly - is that no faradaic processes take place and as such the energetic losses related to electrochemical reactions can be limited ${ }^{12}$.

When using the oxygen/water redox couple as counter electrode reaction, oxygen is anodically produced during charging of the MRB in the oxygen evolution reaction (OER). During discharging, oxygen is reduced again in the oxygen reduction reaction (ORR). Main advantage of this reaction is the resulting theoretical discharge voltage of $1.11 \mathrm{~V}$, which is significantly higher than the maximum discharge voltage of $0.65-0.7 \mathrm{~V}$ obtained when ferricyanide is used ${ }^{13},{ }^{14}$.

In this manuscript, we have operated duplicate MRBs, one with a capacitive and one with an oxygen/water redox counter electrode during 11 and 15 cycles, respectively. Performance of these systems has been analysed in terms of power and energy density and Coulombic and voltage efficiency.

\subsection{MATERIALS AND METHODS}

\subsubsection{General MRB design}

Two MRB systems were constructed for each type of counter electrode, i.e. testing each type in duplicate. A schematic overview of the constructed systems, one with the capacitive and one with the $\mathrm{O}_{2} / \mathrm{H}_{2} \mathrm{O}$ redox counter electrode, are visualized in Figure 1 and Figure 2. For both systems, the counter electrode was placed in between the biocathode and bioanode, and the compartments were separated by a cation exchange membrane (projected surface area of $22 \mathrm{~cm}^{2}$, Nafion ${ }^{\circledR}$ 117). The bioanodes and biocathodes both consisted of graphite felt (3mm, FMI Composites Ltd., Galashiels, Scotland), in which electro-active biofilms were pregrown (see section 'microbial inoculum'). Five layers of graphite felt were used for each electrode, filling the flow compartments completely (inner dimensions $110 \times 20 \times 15 \mathrm{~mm}, 33 \mathrm{~cm}^{3}$ ). As current collector for both biocathode and bioanode, a layer of graphite paper was placed on a solid stainless steel plate (SS316). The felt was firmly pressed against the graphite paper/stainless steel assembly once systems were closed, assuring proper electrical contact. 


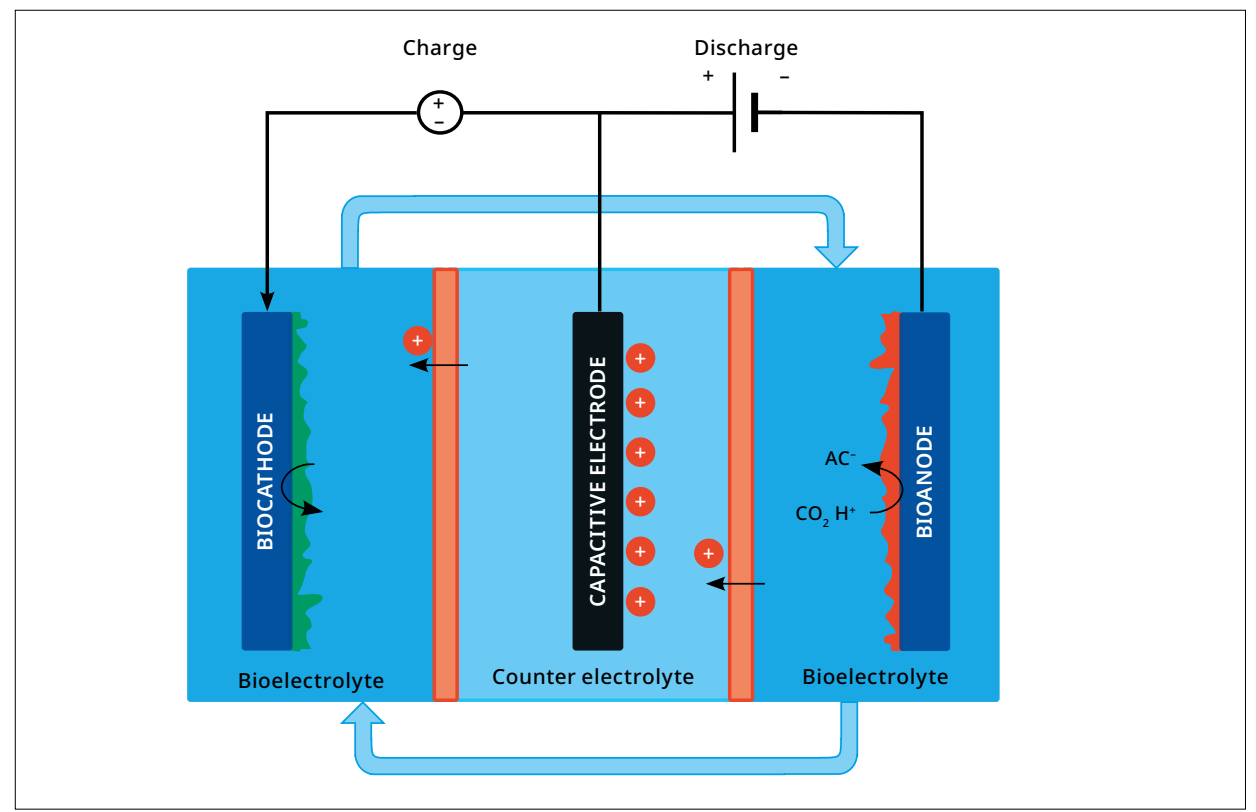

FIGURE 1. | Schematic drawing of the MRB equipped with a capacitive counter electrode. The counter electrode is sandwiched between the biocathode (left) and bioanode (right), and is hydraulically separated from the bioelectrolyte by two cation exchange membranes (yellow). Bioelectrolyte is recirculated over the two biological electrode compartments by peristaltic pumping. When charging, a current is applied between biocathode and counter electrode, while at discharging a positive cell voltage is maintained to deliver a controlled current from bioanode to counter electrode.

The electrolyte, shared between bioanode and biocathode (further referred to as bioelectrolyte), was continuously recirculated between the biocathode and bioanode flow compartments by a peristaltic pump (Masterflex, Canada). O-rings and silicon gaskets sealed the stacked elements and the entire cell was closed with rubber-coated bolts.

The systems were temperature controlled at $30 \pm 2^{\circ} \mathrm{C}$ at all times. Potentials of all electrodes were measured and reported against reference electrodes $(\mathrm{Ag} / \mathrm{AgCl}$, Prosens, Oosterhout, The Netherlands; $+0.203 \mathrm{~V}$ vs SHE) and galvanically connected to the electrolytes adjacent to the electrodes using a Haber-Luggin capillary filled with $3 \mathrm{M} \mathrm{KCl}$. All cell voltages and electrode potentials were monitored and data were collected with a data logger (RSG40, Endress+Hauser, Reinach, Switzerland). The $\mathrm{pH}$ of the bioelectrolyte was measured in-line (CP571D-7BV21, Endress+Hauser, Gerlingen, Germany). Any gas production at the bioelectrodes was monitored with a gas bubble counter (MGC, Ritter Apparatebau, Bochum, Germany). All electrochemical methods were applied and recorded with a potentiostat (N-stat DC, Ivium Technologies, Eindhoven, The Netherlands). 


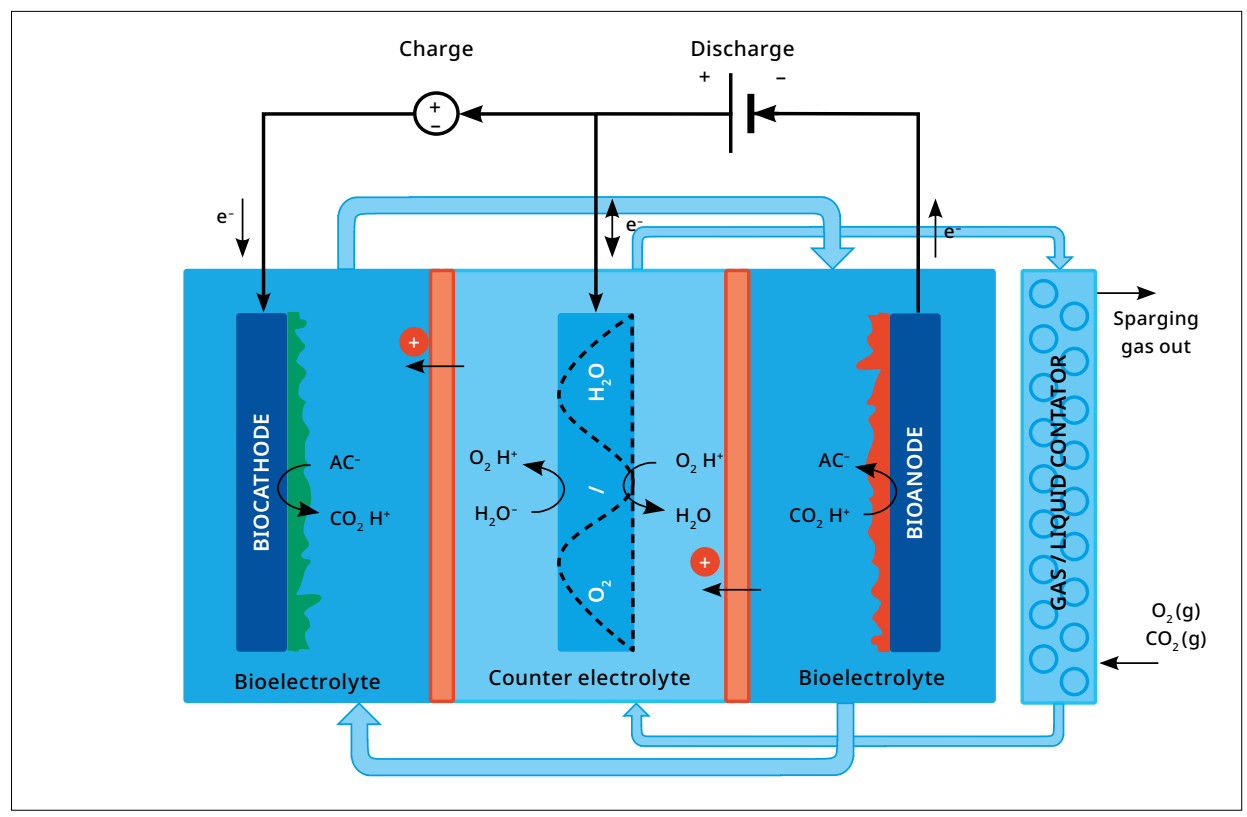

FIGURE 2 | Schematic drawing of the MRB equipped with an $\mathrm{O}_{2} / \mathrm{H}_{2} \mathrm{O}$ counter electrode. The curved dashed line in the middle compartment represents the $\mathrm{Pt} / \mathrm{IrO}_{2}$ catalyzed titanium mesh that was shaped to fill the counter electrode compartment while supporting the two cation exchange membranes (yellow). The counter electrolyte was recirculated over the gas/liquid contactor depicted on the right, where $\mathrm{O}_{2}$ and $\mathrm{CO}_{2}$ were added by mass flow controllers.

\subsubsection{Capacitive counter electrode}

The capacitive counter electrode consisted of a flow compartment completely filled with activated carbon granules (Norit ${ }^{\circledR}$ PK 1-3, from peat, steam activated). Two titanium mesh electrodes ( $2 \mathrm{~mm}$ thickness) were used as current collectors, one at each side of the capacitive electrode, firmly contacting the granules while allowing ionic conductivity towards both bioanode and biocathode. Prior to use, granules were soaked in the to-be-used electrolyte and degassed for 1 hour. The electrolyte in the capacitive flow chamber was recirculated continuously over a small glass compartment positioned above the flow compartment, thus allowing any produced gases to leave the electrolyte and guaranteeing the granules to be gas-free at all times. Prior to use, the capacitive granules were weighed and capacitance was determined over multiple abiotic tests (see Supporting Information). Within the tested potential range, a net capacitance of 481 Farad for the capacitive electrodes was achieved based on a specific capacity of $65 \mathrm{~F} \cdot \mathrm{g}^{-1}$ (dry weight) and a weight per electrode of $7.4 \mathrm{~g}$. 


\subsubsection{Oxygen/water counter electrode}

The bi-directional oxygen reduction and water oxidation counter electrode (further referred to as the $\mathrm{O}_{2} / \mathrm{H}_{2} \mathrm{O}$ counter electrode) consisted of a Pt:IrO $\mathrm{I}_{2}$ coated titanium mesh which was shaped to fill the counter electrode compartment and keep the bioanode and biocathode in place. The counter electrolyte was continuously recirculated over a glass column filled with glass beads ( $4 \mathrm{~mm}$ diameter), serving as gas/liquid contactor. Mass flow controllers were set to continuously sparge the counter electrolyte with $2.5 \mathrm{~mL} \cdot \mathrm{min}^{-1} \mathrm{CO}_{2}$ at an inlet situated at the bottom of the column. Additional relay-actuated mass flow controllers provided a coflow of pure oxygen $\left(0.6 \mathrm{~mL} \cdot \mathrm{min}^{-1}\right)$ during the periods when cells discharged. The oxygen concentration in the counter electrolyte was measured in-line using an amperometric oxygen probe (Endress+Hauser, Oxymax COS22D, Gerlingen, Germany) placed between the electrode flow compartment and the gas/liquid contactor.

\subsubsection{Media composition}

The bioelectrolyte consisted of $0.4 \mathrm{~g} \cdot \mathrm{L}^{-1} \mathrm{NH}_{4} \mathrm{HCO}_{3}, 0.05 \mathrm{~g} \cdot \mathrm{L}^{-1} \mathrm{Ca}(\mathrm{OH})_{2}, 0.1 \mathrm{~g} \cdot \mathrm{L}^{-1} \mathrm{MgSO}_{4}$ $7 \mathrm{H}_{2} \mathrm{O}, 9.6 \mathrm{~g} \cdot \mathrm{L}^{-1} \mathrm{~K}_{2} \mathrm{HPO}_{4}, 2.1 \mathrm{~g} \cdot \mathrm{L}^{-1}$ Sodium 2-bromoethanesulfonate (Na-2-BES), 4.0 $\mathrm{g} \cdot \mathrm{L}^{-1} \mathrm{NaOH}, 0.1 \mathrm{~mL} \cdot \mathrm{L}^{-1}$ trace metals, and $0.1 \mathrm{~mL} \cdot \mathrm{L}^{-1}$ vitamins (DSMZ medium 141) Before use, the solution was saturated with $\mathrm{CO}_{2}$, with a resulting $\mathrm{pH}$ of 7 . The electrolyte used at the capacitive counter electrode was identical in composition to the bioelectrolyte solution, with as exceptions that Na-2-BES, trace metals and vitamins were omitted. Electrolyte used at the $\mathrm{O}_{2} / \mathrm{H}_{2} \mathrm{O}$ counter electrode consisted of a $\mathrm{CO} 2$ saturated $100 \mathrm{mM}$ potassium-phosphate solution buffering at $\mathrm{pH}$ 7. All electrolytes were recirculated at $50 \mathrm{~mL} \cdot \mathrm{min}^{-1}$.

\subsubsection{MRB inoculum}

Individual biocathodes were started 3 and bioanodes were started 2 weeks prior to assembly of the MRBs. These biocathode and bioanode cells were assembled and operated as described earlier for the $\mathrm{MRB}^{3}$. Both biocathodes and bioanodes were continuously fed with fresh bioelectrolyte at $0.125 \mathrm{~mL} \cdot \mathrm{min}^{-1}$. For the bioanode, the bioelectrolyte was complemented with $10 \mathrm{mM}$ sodium acetate. Bioanodes were controlled at a potential of $-0.35 \mathrm{~V}$ while the biocathodes were initially controlled at a current of $-4.55 \mathrm{~A} \cdot \mathrm{m}^{-2}$ for 4 days and then changed to a current density of $-9.09 \mathrm{~A} \cdot \mathrm{m}^{-2} .1 \mathrm{~mL}$ of a $50 \mathrm{vol} \%$ mixture of anaerobic sludge from the municipal wastewater treatment plant in Leeuwarden and cow manure was used as inoculum for the biocathodes and 5-20 mg (wet weight) biomass from previously operated acetate-oxidizing anodes was used to inoculate the anodes. Daily measurements of the VFA concentrations in the bioelectrolyte were performed in the week before 
the electrodes were inoculated in the MRB to confirm efficient production of acetate for the biocathodes. Prior to the assembly of the MRBs, these pre-cultured bioanodes and biocathodes obtained stable Coulombic efficiencies (CE) up to $95 \%$, and biocathodes converted electrons to acetate at potentials between $-0.9 \mathrm{~V}$ and -1.1 $\mathrm{V}$. To assemble the MRBs, the biocathode and bioanode systems were dissembled and bioelectrodes were transferred to the MRBs.

\subsubsection{MRB start-up and operation}

Directly after transferring the bioelectrodes, the MRBs were filled with electrolyte and recirculation was started to remove air. Before the actual experiment was started, systems were alternately charged/discharged every 30 minutes as a pre-treatment, for 5 consecutive days. During this pre-treatment period, biocathodes were current-controlled at $-2.27 \mathrm{~A} \cdot \mathrm{m}^{-2}$ during the charging period, while the anode was at OCP. During the subsequent discharging period, anodes were current-controlled at $4.55 \mathrm{~A} \cdot \mathrm{m}^{-2}$ and biocathodes were at OCP. In case the anode potential increased to values higher than $-350 \mathrm{mV}$, the potentiostat switched to control the potential at this value, this to avoid undesired oxidative processes when acetate was depleted. A small continuous feed of $0.06 \mathrm{~mL} \cdot \mathrm{min}$ ${ }^{1}$ of fresh bioelectrolyte was administered to the systems to assure sufficient macronutrients and inorganic carbon for a good recovery of both bioanodic and biocathodic functions. Additionally, for the capacitive counter electrodes, Coulombic losses occurring throughout charging/discharging led to asymmetric currents, with the overall electrode potential steadily increasing over time. Thus, in the last day of pre-treatment, a dose of acetic acid was administered to the bioanode to poise and bring down the potential of the capacitive electrodes to a potential of approximately $0 \mathrm{~V}$ at the end of the last discharge cycle.

Timing for charging and discharging was set to 16 and 8 hours to mimic a daynight rhythm. As it turned out after the first cycle, the $\mathrm{O}_{2} / \mathrm{H}_{2} \mathrm{O}$ counter electrodes could not sustain reductive currents of $4.55 \mathrm{~A} \cdot \mathrm{m}^{-2}$ at sufficiently low overpotential, yielding negative discharge cell voltages (for more details see Results section). For this reason, a lower current of $-1.15 \mathrm{~A} \cdot \mathrm{m}^{-2}$ (charge) and $2.3 \mathrm{~A} \cdot \mathrm{m}^{-2}$ (discharge) was applied to these systems in the following cycles.

System performance was monitored in detail during the first 11 cycles for the capacitive counter electrode, and the first 15 cycles for the $\mathrm{O}_{2} / \mathrm{H}_{2} \mathrm{O}$ counter electrode. After these cycles, the behavior of both systems did not change considerably, and experiments were ended. In case of the capacitive systems, bioelectrodes were harvested for microbial community analysis. 


\subsubsection{Microbial community analysis}

Samples for microbial community analysis were taken from the systems equipped with capacitive counter electrodes after runs had been completed. Bioelectrodes were taken out of the assembly, put in 50mL tubes, snap-frozen in liquid nitrogen and stored at -80 degrees until further processed. Bacterial/archaeal community analysis on these samples was performed using high-throughput 16S rRNA gene sequencing in order to obtain relative abundances for taxonomic assignments to Operational Taxonomic Units (OTUs). Details on the materials and methods for DNA extraction and microbial community analysis are reported in the Supporting Information.

\subsubsection{Chemical analysis and calculations}

Current and power densities are normalized to both projected membrane surface area $\left(22 \mathrm{~cm}^{2}\right)$ and bioelectrode volume $\left(33 \mathrm{~cm}^{3}\right)$. Energy and charge densities were normalized to bioelectrolyte recirculation volume $(300 \mathrm{~mL})$ to allow comparison with previously studied setups.

To determine the individual CE of the anode and cathode, the bioelectrolyte was sampled 15 minutes before the end of each charge and discharge period. The sampling volume, $1 \mathrm{~mL}$, was replaced with an equal amount of $\mathrm{CO}_{2}$ saturated medium. Samples were analyzed for VFA content using a Dionex UHPLC system equipped with a Phenomenex Rezex Organic Acid $\mathrm{H}+300 \times 7.8 \mathrm{~mm}$ column, with a lower detection limit for formate and acetate of $0.5 \mathrm{mg} \cdot \mathrm{L}^{-1}$ and $1 \mathrm{mg} \cdot \mathrm{L}^{-1}$ for subsequent VFAs (propionate, butyrate etc.) CE for the anode, cathode and for the total system were calculated as

$$
\begin{aligned}
& C E_{a n}=\frac{\int_{16}^{24} I d t}{\Delta c_{V F A} \cdot V \cdot n \cdot F} \\
& C E_{\text {cat }}=\frac{\Delta c_{V F A} \cdot V \cdot n \cdot F}{\int_{0}^{16} I d t} \\
& C E_{\text {total }}=\frac{\int_{16}^{24} I d t}{\int_{0}^{16} I d t}
\end{aligned}
$$

Where I is the current (A), is the concentration change in volatile fatty acids over the measured time interval $\left(\mathrm{mol} \cdot \mathrm{L}^{-1}\right.$, separate calculations were made in case multiple VFAs were detected), $\mathrm{V}$ is the total recirculation volume (300 $\mathrm{mL})$, $\mathrm{n}$ the number of electrons involved in the oxidation or reduction reaction (8 for acetate, 2 for formate, 12 for succinate) and $\mathrm{F}$ is the Faraday constant (96485 $\left.\mathrm{C} \cdot \mathrm{mol}^{-1}\right)$. 
The overall energy efficiency (EE; \%) was calculated as

$$
E E=\frac{\int_{16}^{24} P d t}{\int_{0}^{16} P d t}
$$

Where $P$ is the electrical power applied/obtained during charging/discharging (W). The nominal voltage efficiency (VE) was defined as ratio between the overall energy efficiency and the

$$
\mathrm{VE} \quad=\frac{E E}{C E}
$$

\subsection{RESULTS AND DISCUSSION}

As the results of the duplicate experiments were similar, figures only depict data for a single capacitive and oxygen redox system, allowing comparison between the two types while keeping data presentation comprehensible. Results from the duplicate experiments are shown in the Supporting Information. Across-cycle Coulombic and energy efficiencies for both systems are presented in Figure 3, while Figure 4 gives a more detailed analysis of the electrochemical charge/ discharge behavior in one single cycle.

\subsubsection{Across-cycle: Coulombic and energy efficiencies for both counter electrodes}

Prior to the assembly of the MRBs, the pre-cultured bioanodes and biocathodes obtained stable CEs up to 95\%. In the initial charging/discharging cycles however, CEs for both biocathodes and bioanodes were considerably lower, between $60 \%$ and $80 \%$ for both capacitive counter electrode and $\mathrm{O}_{2} / \mathrm{H}_{2} \mathrm{O}$ counter electrode experiments (Figure 3A and C). A pronounced difference between the two systems was observed in how CE developed over the cycles that followed. For the MRB with the capacitive counter-electrode (Figure 3A), the first two cycles showed relatively low overall CEs (45-50\%), progressively leading to slightly higher CEs around 55-60 \% in subsequent cycles. Combined with VEs of around 40-50 \% (described in more detail in the following section), this led to energy efficiencies of around 25-28 \% from the third cycle onwards. Although the CEs in later cycles for these systems were still lower than those observed during pre-culturing of the electrodes, they were comparable with results obtained using ferro/ferricyanide at the counter electrode ${ }^{3}$.

For the MRB with the $\mathrm{O}_{2} / \mathrm{H}_{2} \mathrm{O}$ counter electrode (Figure 3C), overall CEs dropped steeply during the first cycles, starting at 45-55\% in the first two cycles, to stabilize at $17-25 \%$ at later cycles. Due to large overpotentials occurring at the counter electrode during both charge and discharge, overall VEs for these systems 

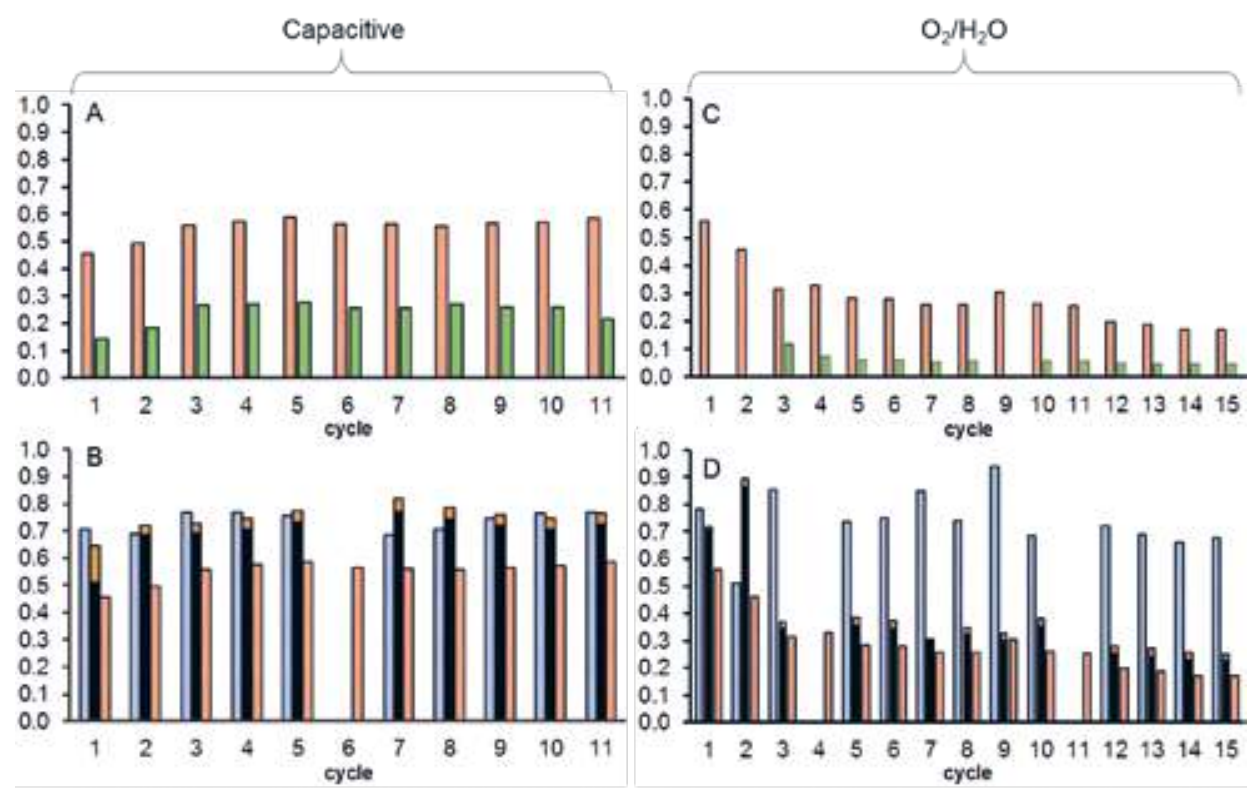

aoverall Coulombic efficiency

danode CE

- cathode CE acetate acathode CE formate

FIGURE 3 | Bar plots of the obtained cycle efficiencies for capacitive (left, A,B) and oxygen/water redox counter electrode equipped (right, C,D) systems. Upper graphs depict the overall energy efficiency and Coulombic efficiency, while lower graphs show specific anodic and cathodic Coulombic efficiencies, with cathodic efficiency specified per VFA (formate, acetate). During certain cycles no VFA data were available (namely cycle 6 for the capacitive, and cycles 4 and 11 for $\mathrm{O}_{2} / \mathrm{H}_{2} \mathrm{O}$ counter electrode) and thus only overall Coulombic efficiency is reported for these cycles.

were substantially lower (10 - $20 \%$ ) than for the capacitive counter electrode and previously tested ferro/ferricyanide counter electrode. Combined with the lower CEs of both bioanode and biocathode, this resulted in low overall energy efficiencies of 2 - $5 \%$. In addition to these overall trends, some irregularities occurred during the $\mathrm{O}_{2} / \mathrm{H}_{2} \mathrm{O}$ experiments, with currents being too high for the counter electrode to sustain positive cell voltage during the first cycle, resulting in a negative EE. At the start of the second cycle for the $\mathrm{O}_{2} / \mathrm{H}_{2} \mathrm{O}$ experiment there was still acetate left-over from the pre-treatment dose administered, and thus no $\mathrm{EE}$ is reported for this cycle. At the onset of discharging during the ninth cycle, the potentiostat switched to potential control immediately due to slow response characteristics of the biofilm. This resulted in higher discharge currents and accompanying negative cell voltages, leading to negative EE for this cycle. Premature switching of the potentiostat was prevented from cycle 10 onwards. 
Figure 3B depicts a more detailed overview of the CE for the MRB with the capacitive counter electrode, showing for bioanode and biocathode separately. The bioanode of the capacitive electrode started at a CE of $70 \%$ in the first cycle to show a slight increase towards $75 \%$ by the $11^{\text {th }}$ cycle. The biocathode in this system started at a CE of $65 \%$ in the first cycle and increased slightly to $74 \%$. Being very similar, bioanode and biocathode processes contributed almost equally to overall Coulombic losses.

Figure 3D shows the $\mathrm{CE}$ for the $\mathrm{MRB}$ with the $\mathrm{O}_{2} / \mathrm{H}_{2} \mathrm{O}$ counter electrode. The bioanodes of the $\mathrm{O}_{2} / \mathrm{H}_{2} \mathrm{O}$ counter electrode equipped systems showed relatively stable CEs ranging between 67 and $90 \%$ throughout the cycles, and were as such performing similarly to bioanodes in the capacitive systems. The biocathode CEs however, while starting at levels of 70-90\% in the first two cycles, lowered drastically in the cycles that followed, to $25 \%$ in the last cycles.

The main difference between the capacitive and $\mathrm{O}_{2} / \mathrm{H}_{2} \mathrm{O}$ counter electrode equipped systems during charging was the presence of oxygen in the counter electrode compartment of the latter. We therefore attribute the low biocathode $\mathrm{CE}$ for the $\mathrm{O}_{2} / \mathrm{H}_{2} \mathrm{O}$ counter electrode systems to oxygen crossover from the counter electrolyte to the bioelectrolyte.

For the $\mathrm{O}_{2} / \mathrm{H}_{2} \mathrm{O}$ counter electrode systems, the biocathode $\mathrm{CE}$ (during charging) was three to four times lower than the bioanode CE (during discharging), this despite oxygen concentrations in the counter electrolyte, and thus presumably also oxygen crossover, being highest during discharging. The lower CE during charging can be explained by the larger overpotential for oxygen reduction at the biocathode during charging, when compared to the bioanode (which is about $0.4-0.6 \mathrm{~V}$ higher than during discharging).

Looking more closely at the volatile fatty acids that were produced in the biocathode (Figure 3B and D), in general, the biocathode used the largest fraction of charge to produce acetate for both counter electrodes. However, also a small fraction of formate was detected.

Production of formate accounted for $2-3 \%$ of the biocathode $\mathrm{CE}$ throughout all cycles of the $\mathrm{O}_{2} / \mathrm{H}_{2} \mathrm{O}$ counter electrode equipped MRBs, while for the capacitive systems $4-10 \%$ of cathodic current was directed to production of formate. Production of formate requires a more negative potential compared to reduction towards acetate ${ }^{6}$. Looking at the overall energetics of the battery this can be beneficial, since the subsequent anode oxidation reaction can take place at a more negative potential, leading to a higher cell voltage during discharge. However, formate concentrations as they were measured in current experiments $( \pm 2-5$ $\mathrm{mg} \cdot \mathrm{L}^{-1}$ ) now played predominantly an intermediate role and as such did not affect overall battery performance substantially. 


\subsubsection{Within-cycle: electrochemical charging and discharging dynamics}

The dynamics occurring in a single charging/discharging cycle, showed considerable changes over the initial three cycles, after which cycles became more comparable to each other. Figure $\mathbf{4}$ shows charging/discharging characteristics (current, cell voltage, electrode potentials and power) for both an $\mathrm{O}_{2} / \mathrm{H}_{2} \mathrm{O}$ counter electrode and capacitive counter electrode equipped system. These characteristics are based on the $11^{\text {th }}$ cycle, because at this point initial fluctuations had dampened out, and thus can be considered representative for longer-term characteristics.

\subsubsection{Charging of the MRB with capacitive counter electrode}

Current densities, electrode potentials and cell voltages, and accompanying power densities for the capacitive counter electrode, are shown in Figure 4A,B and C respectively. From these figures it can be inferred that the capacitive counter electrode acts as a nearly ideal capacitor during charging: when the system is controlled at a constant current during the 16 hours of charging, a near-linear increase of the potential of the capacitive counter electrode was observed, starting from around $+0.15 \mathrm{~V}$ to become $+0.45 \mathrm{~V}$ at the end of the charging cycle. The fact that electrode potential for the counter electrode showed such a consistent response to exposed current provides a good indication for the absence of any substantial and undesired redox reaction to take place within the conditions tested. Also, the absence of any sudden potential change upon onset of charging indicated no mass transfer limitations occurred. Regarding the biocathodes, charging resulted in a potential around $-1.05 \mathrm{~V}$. This corresponds to the potential at which formation of hydrogen can be expected when graphite is used as an electrode and under the conditions applied here. It was also comparable to potentials observed during the pre-culturing of the biocathodes, during which biocathodes were able to produce acetate at potentials between $-0.9 \mathrm{~V}$ and $-1.1 \mathrm{~V}$ (data not shown). Overall, an average cell voltage of $1.5 \mathrm{~V}$ was required during charging.

During charging the bioanodes were not connected, thus left at OCP, with their potentials slowly decreasing from $-0.55 \mathrm{~V}$ to $-0.6 \mathrm{~V}$. With this potential being well below the reversible potential for acetate oxidation, this indicates that the more reductive compounds like hydrogen and formate could also be oxidized by the bioanodes, and that suitable electron transfer mechanisms were in place to do so at a lower electrode potential than used for oxidation of acetate (this in contrast to earlier observations done on hydrogen oxidizing bioanodes as reported by Ntagia et a ${ }^{16}$ ).

\subsubsection{Discharging of the MRB with capacitive counter electrode}

During discharge, bioanodes were operated at constant current at first, leading to an initial anode potential of $-0.42 \mathrm{~V}$. Similar to when charging, during this period of constant current, the capacitive counter electrode showed a near-linear 

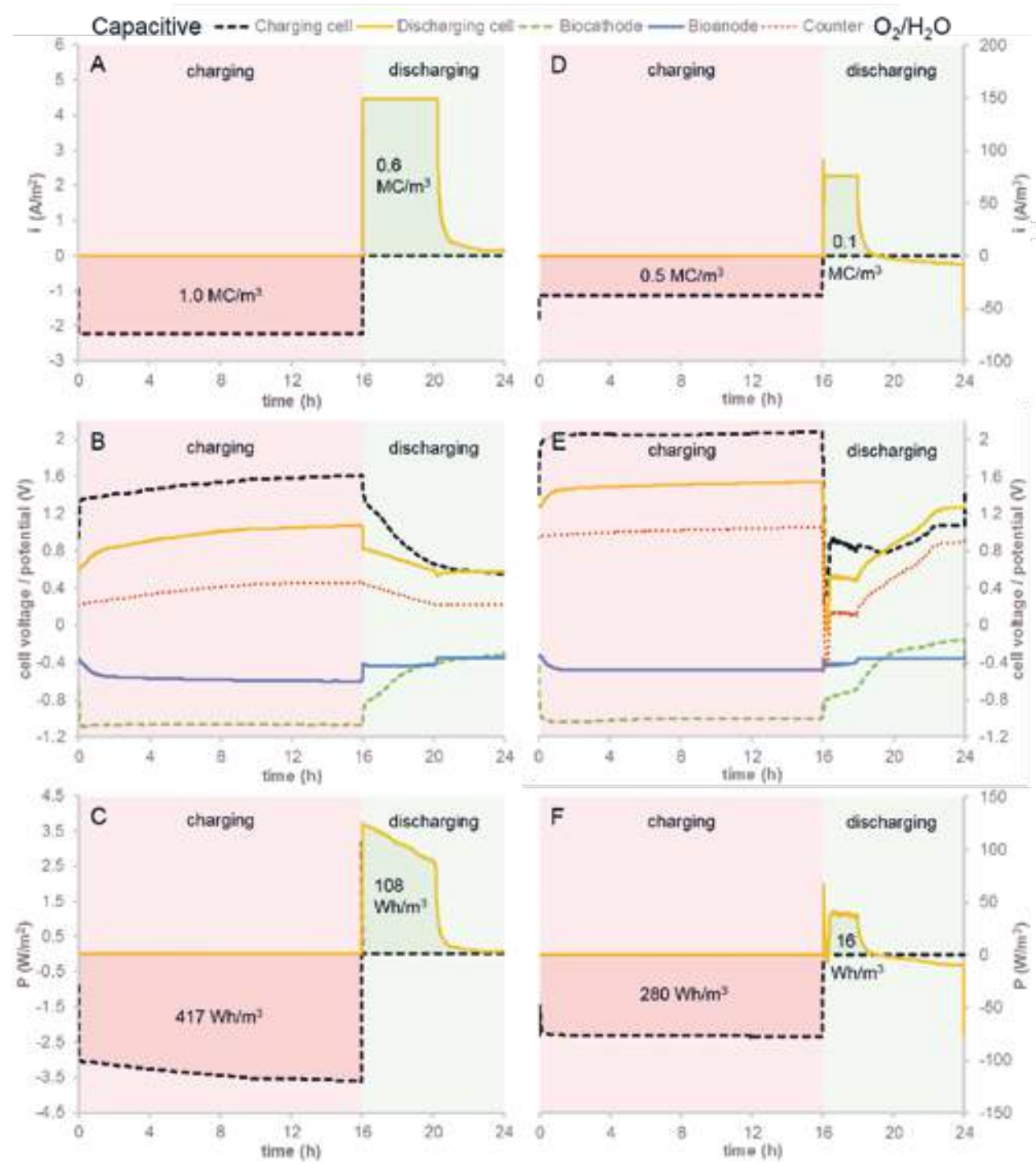

FIGURE 4 | Charging/discharging curves within the 11th cycle of experiments depicting current density, cell voltage, electrode potentials and power density for both systems equipped with a capacitive counter electrode (left, A,B,C) and oxygen/water counter electrodes (right, D,E,F).

decrease in potential. The potential of the capacitive electrode was $+0.45 \mathrm{~V}$ at the start of the discharge period and returned closely to its initial potential of +0.15 V. The difference between the bioanode and capacitive counter electrode potential during discharge led to a nominal discharge (cell) voltage of $+0.7 \mathrm{~V}$. After a period 
of relatively constant bioanode potential, substrate started to deplete, upon which bioanode potential rapidly increased to $-0.35 \mathrm{~V}$. At this point, the potentiostat switched from current to potential control and bioanodes were controlled at $-0.35 \mathrm{~V}$ for the remaining time of discharge, as done previously ${ }^{3}$. During this remaining period, a negligible amount of charge was transferred and the potential of the counter electrode remained stable.

Biocathodes were disconnected during discharge. Upon disconnection, biocathode OCP showed a nearly instant increase towards $-0.6 \mathrm{~V}$, which corresponds well to the reversible potential for hydrogen/formate oxidation. This initial increase was then followed by a gradual increase towards the bioanode potential, thus nearly reaching $-0.35 \mathrm{~V}$ at the end of the discharging period. This observation can be explained by the bioanodic processes gradually depleting the electrolyte from reductive compounds, and the biocathode equilibrating well with the overall redox state of the electrolyte.

\subsubsection{Charging of the MRB with oxygen/water counter electrode}

The experimental details of the 11th cycle of the $\mathrm{O}_{2} / \mathrm{H}_{2} \mathrm{O}$ counter electrode are presented in Figure 4D, E and F. As can be seen in Figure 4D, currents applied in the $\mathrm{O}_{2} / \mathrm{H}_{2} \mathrm{O}$ counter electrode equipped MRB were half those as applied to the capacitive counter electrode equipped systems. This was due to unexpected behavior observed for the $\mathrm{O}_{2} / \mathrm{H}_{2} \mathrm{O}$ counter electrode in the first cycle. Polarization curves taken before the actual experiments started, demonstrated that the counter electrode could sustain a discharging current of $5 \mathrm{~A} \cdot \mathrm{m}^{-2}$ at a potential of $0 \mathrm{~V}$, provided that the oxygen concentration was maintained above $4.4 \mathrm{mg}$ - $\mathrm{L}$ (See Supporting information). Neglecting internal resistances in the system, this current density would theoretically result in a positive discharging cell voltage of $0.46 \mathrm{~V}$, with maximum power densities of approximately $5 \mathrm{~W} \cdot \mathrm{m}^{-2}$. However, during the first cycle's discharge period, the counter electrode potential went down to $-0.55 \mathrm{~V}$ at a current density of $5 \mathrm{~A} \cdot \mathrm{m}^{-2}$, at which point the platinized electrode probably started to generate hydrogen. Whether this was caused by a lesser availability of oxygen or decreased performance of the used catalyst under the present conditions tested was unknown. Even so, the large overpotentials observed resulted in a slightly negative discharging cell voltage of $-0.075 \mathrm{~V}$, and the MRB was thus not functioning as a battery any longer. For this reason, a reduced current of $-1.15 \mathrm{~A} \cdot \mathrm{m}^{-2}$ (charging) and $2.3 \mathrm{~A} \cdot \mathrm{m}^{-2}$ (discharging) was applied in the following cycles.

Applying these lower currents, Figure 4E shows biocathode potential stabilized at $-1 \mathrm{~V}$ during the 16 hour charging period. At the same time, the bioanode potential remained at OCP of $-0.45 \mathrm{~V}$. This poses a slight difference with the MRBs with capacitive counter electrode, in which bioanode OCP during charging reached lower values of $-0.6 \mathrm{~V}$, and may be a direct effect of the presumed oxygen crossover. While charging, the potential of the oxygen redox electrode 
increased to approximately $+1 \mathrm{~V}$. Combined with the biocathode, this overpotential of approximately $0.4 \mathrm{~V}$ for the production of oxygen resulted in a cell voltage of 2 $\mathrm{V}$ during charging.

\subsubsection{Discharging of the MRB with oxygen/water counter electrode}

During the subsequent 8 hour discharging phase, bioanodes were operated at a constant current density of $2.3 \mathrm{~A} \cdot \mathrm{m}^{-2}$ at a nominal potential of $-0.43 \mathrm{~V}$. Anode potentials showed a marked increase in value indicating substrate depletion and therefore anodes were switched from controlled current to control potential at $-0.35 \mathrm{~V}$ once it reached this point. During discharge, biocathode OCP gradually increased to potentials as high as $-0.15 \mathrm{~V}$ as oxidizable substrate depleted. This elevated potential strongly indicates the crossover of oxygen to the bioelectrolyte, and this was further strengthened by the observation of small reductive currents for the bioanode after substrate was depleted and potential was controlled.

To provide the counter electrode with sufficient oxygen during discharging, oxygen was supplied to the system during the discharge period. Despite the supply of oxygen starting immediately upon discharging through the action of potentiostat controlled solenoid valves, oxygen concentration in the counter electrolyte still dropped towards $0.4 \mathrm{mg} \cdot \mathrm{L}^{-1}$ in the first 7-8 mins of the discharging cycle, after which it increased rapidly to reach a concentration around $7 \mathrm{mg} \cdot \mathrm{L}^{-1}$ at the end of the cycle. This initial temporary drop in oxygen was likely caused by a "dead volume" of gas residing in the tubing of the oxygen control system, causing a time delay before oxygen was effectively released into the counter electrolyte. After the initial low oxygen lag phase, stable counter electrode potentials of around $0 \mathrm{~V}$ were obtained, resulting in discharging voltages of $0.42 \mathrm{~V}$ and power densities up to $1.2 \mathrm{~W} \cdot \mathrm{m}^{-2}$ (Figure $\mathbf{4 B}$ and C). Subsequently, when acetate was fully depleted, a small negative current of -0.2 to $-0.3 \mathrm{~A} \cdot \mathrm{m}^{-2}$ was observed during potential controlled discharging, indicating reduction of crossover oxygen at the bioanode to be dominant at this point. This reductive current at the bioanode resulted in a potential increase of the counter electrode towards $+1.05 \mathrm{~V}$, and the energy costs involved were not included in the EE calculation (if these would have been included, it would have led to a reduction of EE of only $0.1 \%$ for the presented cycle, this due to the small current).

Similar to the capacitive counter electrode system, in the $\mathrm{O}_{2} / \mathrm{H}_{2} \mathrm{O}$ counter electrode system also considerable overpotentials of $0.54 \mathrm{~V}$ were required at the biocathodes to enable hydrogen and consequent acetate production. These overpotentials are commonly observed in biocathodes and may be due to the necessity for hydrogen production as a mediating step for microbial acetate synthesis ${ }^{17}$. The bioanodes operated close to the theoretical potential with observed overpotentials of $0.13 \mathrm{~V}$. The overpotentials of the oxygen redox counter electrode, with a theoretical reversible potential of $0.59 \mathrm{~V}$, amounted to approximately 0.41 $\mathrm{V}$ during charging and $0.59 \mathrm{~V}$ during discharging. 


\subsubsection{Microbial community diversity}

Figure 5 shows a heat map of the 16S rRNA gene sequencing results with the relative abundance of the taxonomic assignments at the order level. All assigned reads with an abundance greater than $1 \%$ are displayed separately. In case additional taxonomic assignments of OTUs at the family/genus level showed a high abundance of one or few taxonomic groups within an order, these are mentioned in Figure 5 as well.

Overall, Figure 5 shows a clear distinction between the bioanodes and biocathodes from the capacitive systems. Most characteristic for the bioanodes is the dominant occurrence of Geobacter species, as is to be expected and well described previously for these systems ${ }^{18,19}$. Together with species belonging to Desulfovibrionales, direct extracellular electron transfer (EET) from or to an electrode by means of membrane bound cytochromes has been demonstrated for this group of bacteria ${ }^{20,21}$, and their high relative abundance specifically on the bioanode matches with the relatively low overpotentials observed there during discharge of the MRB.

The cathode in turn shows, as most prominent species, those belonging to the Selenomonadales order. Most dominantly represented within this order are species belonging to the genus Sporomusa, which have been previously investigated for their role in acetate producing biocathodes ${ }^{17,22}$. Together with bacteria within the (here less abundant) order of Clostridiales, Selenomonadales comprise a group within which numerous homoacetogenic species reside, and most of the species within these orders are capable of performing the Wood-Ljungdahl pathway for producing acetate from $\mathrm{CO}_{2}$ and $\mathrm{H}_{2}^{22}$. The clear difference in abundance for Selenomonadales between bioanodes and biocathodes found here underpins this role.

On the second place in relative abundance, for both bioanodes and biocathodes, are species belonging to the order of Rhodocyclales, with most OTUs assigned to the genus Dechlorobacter. These have been reported frequently in biocathodes, but are found in higher relative abundance in bidirectional bioelectrodes, initially operated as anodes before being poised more negative potential ${ }^{23-25}$. Species belonging to the orders of Pseudomonales and Burkholderiales, have been described as co-occurring under these conditions, and may play a comparable role $\mathrm{e}^{23,25}$. The association of these groups of bacteria specifically with reversed electrodes seems to provide, although not fully, a basis for constructing a bi-directional bioelectrode, in which the biota present can gain energy both by electrogenic oxidation and electrotrophic reduction. If successfully established, this could reduce the MRBs footprint by integration of bioanode and biocathode. Supportive to this hypothesis is the recent report of such an bi-directional electrode by Yates et al. ${ }^{26}$, in which growth of electrocatalytic biomass was established by polarity inversion on a 10 min interval. It would be interesting to investigate whether this functionality can be maintained over longer charge/discharge intervals. 


\begin{tabular}{|c|c|c|c|c|c|c|}
\hline \multirow{2}{*}{ Order } & \multirow{2}{*}{$\begin{array}{l}\text { most dominant } \\
\text { family/genus within }\end{array}$} & \multicolumn{2}{|c|}{ Bioanode } & \multicolumn{2}{|c|}{ Biocathode } & \\
\hline & & 1 & 2 & 1 & 2 & \\
\hline Bacteroidales & Rikenellaceae & $5 \%$ & $7 \%$ & $4 \%$ & $12 \%$ & \multirow{5}{*}{ Fermentative } \\
\hline Sphingobacteriales & Lentimicrobium sp. & $4 \%$ & $1 \%$ & $4 \%$ & $2 \%$ & \\
\hline Spirochaetales & \multirow[t]{3}{*}{ Sphaerochaeta sp. } & $1 \%$ & $2 \%$ & $1 \%$ & $2 \%$ & \\
\hline Anaerolineales & & $2 \%$ & $0 \%$ & $1 \%$ & $0 \%$ & \\
\hline Mollicutes (class) & & $2 \%$ & $0 \%$ & $5 \%$ & $0 \%$ & \\
\hline Clostridiales & Proteiniborus sp. & $1 \%$ & $5 \%$ & $1 \%$ & $5 \%$ & \multirow{2}{*}{ Homo-acetogenic } \\
\hline Selenomonadales & Sporomusasp. & $12 \%$ & $11 \%$ & $31 \%$ & $30 \%$ & \\
\hline Pseudomonadales & Thiopseudomonas sp. & $3 \%$ & $1 \%$ & $10 \%$ & $6 \%$ & \multirow{3}{*}{$\begin{array}{l}\mathrm{H}_{2} / \mathrm{CO} / \text { formate-based } \\
\text { EET }\end{array}$} \\
\hline Burkholderiales & Hydrogenophaga sp. & $0 \%$ & $0 \%$ & $1 \%$ & $1 \%$ & \\
\hline Rhodocyclales & Dechlorobactersp. & $17 \%$ & $23 \%$ & $21 \%$ & $23 \%$ & \\
\hline Desulfovibrionales & Desulfowibrio sp & $1 \%$ & $2 \%$ & $3 \%$ & $5 \%$ & \multirow{4}{*}{$\begin{array}{l}\text { Cytochrome based } \\
\text { EET }\end{array}$} \\
\hline Desulfuromonadales & Geobactersp. & $43 \%$ & $39 \%$ & $8 \%$ & $4 \%$ & \\
\hline Other & & $7 \%$ & $5 \%$ & $8 \%$ & $8 \%$ & \\
\hline Unidentified & & $2 \%$ & $2 \%$ & $2 \%$ & $2 \%$ & \\
\hline
\end{tabular}

FIGURE 5 | Heat map showing relative abundance of taxonomic assignments of OTUs at the order level as resulted from the $16 \mathrm{~S}$ rRNA sequencing of samples of both bioanodes and biocathodes of the capacitive electrode equipped systems. Results for both duplicates are shown (bioanode/biocathode 1\&2). For the full taxonomic assignment results, see Supporting Information. Provisional functional grouping, depicted on the right, was suggested based on previous reports on mentioned groups and references to these reports are provided in the text.

Species within the orders of Anaerolineales ${ }^{27}$, Spirochaetales ${ }^{28}$, Sphingobacteriales ${ }^{29}$ and Bacteroidales ${ }^{30,31}$, and members of the class Mollicutes ${ }^{32}$, are frequently reported for their role in anaerobic digestion and fermentative properties. With abundances reported here not clearly pointing towards a preference for biocathode or bioanodes, bacteria associated to these groups are therefore more likely to have played a purely heterotrophic role. A side note should be placed here whether they happened to be active at all, which is, given their low relative abundance, questionable. Moreover, although syntrophic interactions between these fermentative heterotrophs and alleged electrogenic/electrotrophic bacteria may be expected ${ }^{33}$, more extensive analysis (such as metatranscriptomics) would be required to further investigate the activities of described bacteria and their interactions.

\subsubsection{Comparison of the counter electrodes tested so far: Implications and future perspective}

Table 1 shows a comparative overview of the obtained and projected theoretical maximally attainable key parameters for the MRB with the three by now tested counter electrodes. Quantities are given regarding Coulombic, voltage and energy 
TABLE 1 | Comparison of the so-far-tested counter electrodes with use in the MRB. Both experimentally acquired values (exp., left numbers) as projected theoretical maximum attainable values (max., right numbers) are displayed.

\begin{tabular}{|l|c|c|c|c|c|c|}
\hline \multirow{2}{*}{} & \multicolumn{2}{|c|}{ Ferro/ferricyanide } & \multicolumn{2}{|c|}{ Capacitive } & \multicolumn{2}{c|}{$\mathrm{O}_{2} / \mathrm{H}_{2} \mathrm{O}$} \\
\cline { 2 - 7 } & exp. & max. & exp. & max. & exp. & max. \\
\hline Coulombic efficiency (\%) & 65 & 80 & 55 & 80 & 25 & 80 \\
\hline Voltage efficiency (\%) & 54 & 63 & 45 & 58 & 15 & 44 \\
\hline Energy efficiency (\%) & 35 & 50 & 25 & 45 & 3.7 & 35 \\
\hline Energy density (W-h·L-1) & 0.1 & 17 & 0.1 & 2.5 & 0.02 & 82 \\
\hline Power density (W.L-1) & 0.2 & 58 & 0.1 & 43 & 0.04 & 31 \\
\hline
\end{tabular}

efficiency, and energy and power densities. Experimentally obtained numbers are taken from existing and presented datasets. For the estimation of theoretical maxima, the following assumptions were made: (1) an achievable volumetric current density of $750 \mathrm{~A} \cdot \mathrm{m}^{-3}$ for all biocatalyzed electrodes at which (2) acetate producing biocathodes are able to be operated at a potential of $-0.87 \mathrm{~V}^{34}$, (3) acetate oxidizing bioanodes to be operated at $-0.42 \mathrm{~V}^{35}$ (C-SS felt, (4) capacitive counter electrodes both during charging and discharging to be operated at a nominal potential of $0.15 \mathrm{~V}$, assuming an operational potential window of 0.6 to $-0.3 \mathrm{~V}$, (5) biocatalyzed oxygen reduction at a potential of $0.3 \mathrm{~V}^{36}(6)$ anodic oxygen evolution at a potential of $1 \mathrm{~V}$ and (7) gaseous oxygen being stored at a molar density of 0.01 $\mathrm{mol} \cdot \mathrm{m}^{-3}$. Coulombic losses are assumed to be limited to $20 \%$ for all types of counter electrode, assuming oxygen crossover to be less problematic once current densities are increased. Charge storage was assumed to take place in the form of acetate, with maximum attainable concentrations of $0.75 \mathrm{M}^{37}$, and in case of ferro/ferricyanide as a counter electrode redox couple, a maximum solubility of $1 \mathrm{M}$ was used.

Finally, for the power density maximum projections, ferro/ferricyanide and capacitive electrodes were assumed to be non-limiting with regard to current density, thus with only the volume of biologically catalyzed electrodes defining attainable power density. Furthermore, no operational energy losses were taken into account.

Looking at Table 1, it is clear that the capacitive counter electrode equipped systems outperformed the MRBs with an $\mathrm{O}_{2} / \mathrm{H}_{2} \mathrm{O}$ counter electrode on all aspects in the current experiments. However, using capacitive electrodes in aqueous environments limits the attainable energy density: restricted in potential window by electrolysis of water, during charging the capacitive electrode potential may not increase much further than $+0.7 \mathrm{~V}$, after which oxygen production (and concomitant oxidation of the carbon compounds) may occur, thus not contributing any longer to double layer polarization. When discharging, the capacitive electrode 
potential may not decrease to values too close to the anode potential (-0.4 V in these experiments) in order to maintain meaningful discharge cell voltages. As such, a theoretical voltage window of $1.1 \mathrm{~V}$ is available to the capacitive electrode which allows for only limited charge densities given the currently obtained capacities around $65 \mathrm{~F} \cdot \mathrm{g}^{-1}$ (dry weight).

With the $\mathrm{O}_{2} / \mathrm{H}_{2} \mathrm{O}$ counter electrode, MRB performance is severely limited by the combined effects of both oxygen crossover, affecting the $\mathrm{CE}$, and the slow reaction kinetics of both the ORR and OER. Especially the large overpotential currently required for ORR is problematic, as this limits the obtainable current and power density during discharging. The relative impact of oxygen crossover on CE may be substantially lowered in case a faster ORR can be established without increasing oxygen concentrations, as the flux of oxygen crossover only depends on concentration gradient, and not on current. A possible future implementation and optimization of biologically catalysed oxygen reduction into the MRB platform might provide possibilities to this extent; with specific current densities of $0.9 \mathrm{~A} \cdot \mathrm{m}^{-2}$ reported for flat graphite plate cathodes polarized at $0.15 \mathrm{~V}^{36}$. When adequately operated on a three-dimensional electrode material, sufficiently high volumetric current densities should be feasible at oxygen concentrations comparable to those used in current experiments. However, this would still not dispel the requirements of precious catalysts for the anode reaction, severely impeding the economic and ecological principles this battery strives for.

The capacitive electrode has proven to be a stable counter electrode in the microbial rechargeable battery. Losses encountered can be mainly attributed to the performance of the biocathode, with lower cycling efficiency compared to the use of ferro/ferricyanide being caused by the biocathodes' overpotential having a relatively larger impact on VE given the slightly lower nominal potential of the capacitive electrode. A reduction in overpotential at the biocathode could improve performance at this point. This is however not foreseen as both from our own experiments and other work ${ }^{17}$ it seems that hydrogen is an inevitable mediating compound in the reduction of $\mathrm{CO}_{2}$ towards carboxylates in BESs at relevant current densities.

Looking for ways to reduce the losses at the biocathodes of the MRB, a theoretical possibility for improvement of the MRB might be the use of formate as main charge storage molecule, which has a lower formal redox potential than acetate, and is readily formed by a wide range of microorganisms. Doing so however would pose a major thermodynamic challenge on the selectivity of electrochemically active biofilms. With formate close to hydrogen in redox potential, large tendencies, especially at higher formate concentrations, would exist to either produce hydrogen or acetate. This is possibly the reason why formate production so far is mainly studied in synthetic biology ${ }^{38}$ although some occurrences of whole cell synthesis have been reported ${ }^{39,40}$. A possible disadvantage in using formate is the lower reduction state of the carbon molecule when compared to acetate, thus leading to lower overall energy density. 
Although by the data presented in the current study the capacitive systems are outperforming the oxygen reduction reaction equipped MRBs in terms of energy efficiency by a factor of 5 , the potential upscaling in terms of energy density is foreseen to be problematic due to relatively low charge capacity associated with EDL charging. With this regard, the use of the $\mathrm{O}_{2} / \mathrm{H}_{2} \mathrm{O}$ electrode may provide a more optimistic outlook, with theoretically attainable energy densities of around $82 \mathrm{Wh} \cdot \mathrm{L}^{-1}$ and a cycling efficiency of up to $38 \%$, under the mentioned assumptions. However also for the $\mathrm{H}_{2} \mathrm{O} / \mathrm{O}_{2}$ electrode, inevitable disadvantages remain, most notably the requirement of precious metal catalysts for performing the OER and the need for oxygen produced during charging to be stored under high pressures, with safety issues and energy losses associated. Given these fundamental aspects that stand in the way of a successful further development of the MRB concept, more promising alternative candidates for a counter electrode may have to be sought for.

\subsection{ACKNOWLEDGEMENTS}

This work was performed in the cooperation framework of Wetsus, European Centre of Excellence for Sustainable Water Technology (www.wetsus.eu). Wetsus is co-funded by the Dutch Ministry of Economic Affairs and Ministry of Infrastructure and Environment, the Province of Fryslân, and the Northern Netherlands Provinces. The authors like to thank the participants of the Resource Recovery research theme for the fruitful discussions and their financial support. 


\subsection{REFERENCES}

(1) Trenberth, K. E.; Fasullo, J. T.; Kiehl, J. Earth's global energy budget. Bull. Am. Meteorol. Soc. 2009, 90 (3), 311-323 DOI: 10.1175/2008BAMS2634.1.

(2) Larcher, D.; Tarascon, J. M. Towards greener and more sustainable batteries for electrical energy storage. Nat. Chem. 2015, 7 (1), 19-29 DOI: 10.1038/nchem.2085.

(3) Molenaar, S. D.; Mol, A. R.; Sleutels, T. H. J. A.; ter Heijne, A.; Buisman, C. J. N. Microbial Rechargeable Battery: Energy Storage and Recovery through Acetate. Environ. Sci. Technol. Lett. 2016, 3 (4), 144-149 DOI: 10.1021/acs.estlett.6b00051.

(4) Rabaey, K.; Rozendal, R. A. Microbial electrosynthesis - revisiting the electrical route for microbial production. Nat. Rev. Microbiol. 2010, 8 (10), 706-716 DOI: 10.1038/nrmicro2422.

(5) Jourdin, L.; Grieger, T.; Monetti, J.; Flexer, V.; Freguia, S.; Lu, Y.; Chen, J.; Romano, M.; Wallace, G. G.; Keller, J. High Acetic Acid Production Rate Obtained by Microbial Electrosynthesis from Carbon Dioxide. Environ. Sci. Technol. 2015, 49 (22), 13566-13574 DOI: 10.1021/acs.est.5b03821.

(6) Molenaar, S. D.; Saha, P.; Mol, A. R.; Sleutels, T. H.J. A.; ter Heijne, A.; Buisman, C. J. N. Competition between methanogens and acetogens in biocathodes: A comparison between potentiostatic and galvanostatic control. Int. J. Mol. Sci. 2017, 18 (1) DOI: 10.3390/ijms18010204.

(7) Logan, B. E.; Hamelers, B.; Rozendal, R.; Schröder, U.; Keller, J.; Freguia, S.; Aelterman, P.; Verstraete, W.; Rabaey, K. Microbial fuel cells: Methodology and technology. Environ. Sci. Technol. 2006, 40 (17), 5181-5192 DOI: $10.1021 /$ es0605016.

(8) Sleutels, T.; Molenaar, S.; Heijne, A.; Buisman, C. Low Substrate Loading Limits Methanogenesis and Leads to High Coulombic Efficiency in Bioelectrochemical Systems. Microorganisms 2016, 4 (1), 7 DOI: 10.3390/ microorganisms4010007.

(9) Luo, X.; Wang, J.; Dooner, M.; Clarke, J. Overview of current development in electrical energy storage technologies and the application potential in power system operation. Appl. Energy 2014, 137, 511-536 DOI: 10.1016/j.apenergy.2014.09.081.

(10) Pharr, C. M.; Griffiths, P. R. Infrared Spectroelectrochemical Analysis of Adsorbed Hexacyanoferrate Species Formed during Potential Cycling in the Ferrocyanide/Ferricyanide Redox Couple. Anal. Chem. 1997, 69 (22), 4673-4679 DOI: 10.1021/ac961120l.

(11) Kötz, R.; Carlen, M. Principles and applications of electrochemical capacitors. Electrochim. Acta 2000, 45 (15-16), 2483-2498 DOI: DOI: 10.1016/S0013-4686(00)00354-6.

(12) Porada, S.; Zhao, R.; Wal, A. Van Der; Presser, V.; Biesheuvel, P. M. Review on the science and technology of water desalination by capacitive deionization. Prog. Mater. ... 2013, 58, 1388-1442.

(13) Shahrezaei, V.; Swain, P. S. Analytical distributions for stochastic gene expression. Proc. Natl. Acad. Sci. 2008, 105 (1), 17256-17261 DOI: 10.1073/pnas.0803850105.

(14) O'Reilly, J. E. Oxidation-reduction potential of the ferro-ferricyanide system in buffer solutions. $B B A$ Bioenerg. 1973, 292 (3), 509-515 DOI: 10.1016/0005-2728(73)90001-7.

(15) Zehnder, A. J. B.; Huser, B. A.; Brock, T. D.; Wuhrmann, K. Characterization of an acetate-decarboxylating, non-hydrogen-oxidizing methane bacterium. Arch. Microbiol. 1980, 124 (1), 1-11 DOI: 10.1007/BF00407022.

(16) Ntagia, E.; Rodenas, P.; Ter Heijne, A.; Buisman, C. J. N.; Sleutels, T. H. J. A. Hydrogen as electron donor for copper removal in bioelectrochemical systems. Int. J. Hydrogen Energy 2016, 41 (13), 5758-5764 DOI: 10.1016/j.ijhydene.2016.02.058. 
(17) May, H. D.; Evans, P. J.; LaBelle, E. V. The bioelectrosynthesis of acetate. Curr. Opin. Biotechnol. 2016, 42, 225-233 DOI: 10.1016/j.copbio.2016.09.004.

(18) Kracke, F.; Vassilev, I.; Krömer, J. O. Microbial electron transport and energy conservation - The foundation for optimizing bioelectrochemical systems. Frontiers in Microbiology. 2015.

(19) Kumar, R.; Singh, L.; Wahid, Z. A.; Din, M. F. M. Exoelectrogens in microbial fuel cells toward bioelectricity generation: A review. Int. J. Energy Res. 2015, 39 (8), 1048-1067 DOI: 10.1002/er.3305.

(20) Kang, C. S.; Eaktasang, N.; Kwon, D. Y.; Kim, H. S. Enhanced current production by Desulfovibrio desulfuricans biofilm in a mediator-less microbial fuel cell. Bioresour. Technol. 2014, 165 (C), 27-30 DOI: 10.1016/j.biortech.2014.03.148.

(21) Lovley, D. R. Powering microbes with electricity: direct electron transfer from electrodes to microbes. Environ. Microbiol. Rep. 2011, 3 (1), 27-35 DOI: 10.1111/j.1758-2229.2010.00211.x.

(22) Nevin, K. P.; Hensley, S. a; Franks, A. E.; Summers, Z. M.; Ou, J.; Woodard, T. L.; Snoeyenbos-West, O. L.; Lovley, D. R. Electrosynthesis of organic compounds from carbon dioxide is catalyzed by a diversity of acetogenic microorganisms. Appl. Environ. Microbiol. 2011, 77 (9), 2882-2886 DOI: 10.1128/AEM.02642-10.

(23) Mateos, R.; Sotres, A.; Alonso, R. M.; Escapa, A.; Morán, A. Impact of the start-up process on the microbial communities in biocathodes for electrosynthesis. Bioelectrochemistry 2018, 121, 27-37 DOI: 10.1016/j. bioelechem.2018.01.002.

(24) Yun, H.; Liang, B.; Kong, D.; Wang, A. Improving biocathode community multifunctionality by polarity inversion for simultaneous bioelectroreduction processes in domestic wastewater. Chemosphere $\mathbf{2 0 1 8 ,}$ 194, 553-561 DOI: 10.1016/j.chemosphere.2017.12.030.

(25) Jourdin, L.; Lu, Y.; Flexer, V.; Keller, J.; Freguia, S. Biologically Induced Hydrogen Production Drives High Rate/High Efficiency Microbial Electrosynthesis of Acetate from Carbon Dioxide. ChemElectroChem 2016, 3 (4), 581-591 DOI: 10.1002/celc.201500530.

(26) Yates, M. D.; Ma, L.; Sack, J.; Golden, J. P.; Strycharz-Glaven, S. M.; Yates, S. R.; Tender, L. M. Microbial electrochemical energy storage and recovery in a combined electrotrophic and electrogenic biofilm. Environ. Sci. Technol. Lett. 2017, 4 (9), 374-379 DOI: 10.1021/acs.estlett.7b00335.

(27) Liang, B.; Wang, L. Y.; Mbadinga, S. M.; Liu, J. F.; Yang, S. Z.; Gu, J. D.; Mu, B. Z. Anaerolineaceae and Methanosaeta turned to be the dominant microorganisms in alkanes-dependent methanogenic culture after long-term of incubation. AMB Express 2015, 5 (1) DOI: 10.1186/s13568-015-0117-4.

(28) Ritalahti, K. M.; Justicia-Leon, S. D.; Cusick, K. D.; Ramos-Hernandez, N.; Rubin, M.; Dornbush, J.; Löffler, F. E. Sphaerochaeta globosa gen. nov., sp. nov. and sphaerochaeta pleomorpha sp. nov., free-living, spherical spirochaetes. Int. J. Syst. Evol. Microbiol. 2011, 62 (1), 210-216 DOI: 10.1099/ijs.0.023986-0.

(29) Sun, L.; Toyonaga, M.; Ohashi, A.; Tourlousse, D. M.; Matsuura, N.; Meng, X. Y.; Tamaki, H.; Hanada, S.; Cruz, R.; Yamaguchi, T.; et al. Lentimicrobium saccharophilum gen. nov., sp. nov., a strictly anaerobic bacterium representing a new family in the phylum bacteroidetes, and proposal of lentimicrobiaceae fam. nov. Int. J. Syst. Evol. Microbiol. 2016, 66 (7), 2635-2642 DOI: 10.1099/ijsem.0.001103.

(30) Morrison, J. M.; Murphy, C. L.; Baker, K.; Zamor, R. M.; Nikolai, S. J.; Wilder, S.; Elshahed, M. S.; Youssef, N. H. Microbial communities mediating algal detritus turnover under anaerobic conditions. Peer 2017, 5 , e2803 DOI: 10.7717/peerj.2803.

(31) Su, X. L.; Tian, Q.; Zhang, J.; Yuan, X. Z.; Shi, X. S.; Guo, R. B.; Qiu, Y. L. Acetobacteroides hydrogenigenes gen. nov., Sp. nov., An anaerobic hydrogen-producing bacterium in the family Rikenellaceae isolated from a reed swamp. Int. J. Syst. Evol. Microbiol. 2014, 64, 2986-2991 DOI: 10.1099/ijs.0.063917-0. 
(32) Lu, L.; Xing, D.; Ren, N. Pyrosequencing reveals highly diverse microbial communities in microbial electrolysis cells involved in enhanced $\mathrm{H} 2$ production from waste activated sludge. Water Res. 2012, 46 (7), 2425-2434 DOI: 10.1016/j.watres.2012.02.005.

(33) Marshall, C. W.; Ross, D. E.; Handley, K. M.; Weisenhorn, P. B.; Edirisinghe, J. N.; Henry, C. S.; Gilbert, J. A.; May, H. D.; Norman, R. S. Metabolic reconstruction and modeling microbial electrosynthesis. Sci. Rep. 2017, 7 (1), 1-12 DOI: 10.1038/s41598-017-08877-z.

(34) Jourdin, L.; Freguia, S.; Flexer, V.; Keller, J. Bringing High-Rate, CO2-Based Microbial Electrosynthesis Closer to Practical Implementation through Improved Electrode Design and Operating Conditions. Environ. Sci. Technol. 2016, 50 (4), 1982-1989 DOI: 10.1021/acs.est.5b04431.

(35) Guo, K.; Hidalgo, D.; Tommasi, T.; Rabaey, K. Pyrolytic carbon-coated stainless steel felt as a highperformance anode for bioelectrochemical systems. Bioresour. Technol. 2016, 211, 664-668 DOI: 10.1016/j. biortech.2016.03.161.

(36) Rothballer, M.; Picot, M.; Sieper, T.; Arends, J. B. A.; Schmid, M.; Hartmann, A.; Boon, N.; Buisman, C. J. N.; Barrière, F.; Strik, D. P. B. T. B. Monophyletic group of unclassified y-Proteobacteria dominates in mixed culture biofilm of high-performing oxygen reducing biocathode. Bioelectrochemistry 2015, 106, 167-176 DOI: 10.1016/j.bioelechem.2015.04.004.

(37) Demler, M.; Weuster-Botz, D. Reaction engineering analysis of hydrogenotrophic production of acetic acid by Acetobacterium woodii. Biotechnol. Bioeng. 2011, 108 (2), 470-474 DOI: 10.1002/bit.22935.

(38) Choe, H.; Joo, J. C.; Cho, D. H.; Kim, M. H.; Lee, S. H.; Jung, K. D.; Kim, Y. H. Efficient CO2-reducing activity of NAD-dependent formate dehydrogenase from Thiobacillus sp. KNK65MA for formate production from CO2gas. PLoS One 2014, 9 (7) DOI: 10.1371/journal.pone.0103111.

(39) Mourato, C.; Martins, M.; da Silva, S. M.; Pereira, I. A. C. A continuous system for biocatalytic hydrogenation of CO2to formate. Bioresour. Technol. 2017, 235, 149-156 DOI: 10.1016/j.biortech.2017.03.091.

(40) Hwang, H.; Yeon, Y. J.; Lee, S.; Choe, H.; Jang, M. G.; Cho, D. H.; Park, S.; Kim, Y. H. Electro-biocatalytic production of formate from carbon dioxide using an oxygen-stable whole cell biocatalyst. Bioresour. Technol. 2015, 185, 35-39 DOI: 10.1016/j.biortech.2015.02.086.

(41) Takahashi, S.; Tomita, J.; Nishioka, K.; Hisada, T.; Nishijima, M. Development of a prokaryotic universal primer for simultaneous analysis of Bacteria and Archaea using next-generation sequencing. PLoS One 2014, 9 (8) DOI: 10.1371/journal.pone.0105592.

(42) Caporaso, J. G.; Kuczynski, J.; Stombaugh, J.; Bittinger, K.; Bushman, F. D.; Costello, E. K.; Fierer, N.; Peña, A. G.; Goodrich, J. K.; Gordon, J. I.; et al. QIIME allows analysis of high-throughput community sequencing data. Nat. Methods 2010, 7, 335.

(43) Quast, C.; Pruesse, E.; Yilmaz, P.; Gerken, J.; Schweer, T.; Yarza, P.; Peplies, J.; Glöckner, F. O. The SILVA ribosomal RNA gene database project: Improved data processing and web-based tools. Nucleic Acids Res. 2013, 41 (D1) DOI: 10.1093/nar/gks1219.

(44) Edgar, R. C. Search and clustering orders of magnitude faster than BLAST. Bioinformatics 2010, 26 (19), 2460-2461 DOI: 10.1093/bioinformatics/btq461.

(45) Wang, Q.; Garrity, G. M.; Tiedje, J. M.; Cole, J. R. Naive bayesian classifier for rapid assignment of rRNA sequencesinto the new bacterial taxonomy. Appl. Environ. Microbiol. 2007, 73, 5261-5267 DOI: 10.1128/ AEM.00062-07. 


\subsection{APPENDICES}

\subsubsection{Characterization of the capacitive electrode}

\subsubsection{Materials and Methods.}

Prior to biotic experiments, two separate cells were assembled for characterization of the capacitive counter electrodes. Each electrochemical cell consisted of two Plexi glass flow compartments, one filled with activated charcoal (AC) granules (Norit®, PK 1-3, from peat, steam activated) and the other filled with plastic spacer material. As a current collector/contactor for the AC granules, a titanium mesh (thickness $2 \mathrm{~mm}$ ) was used. Serving as a counter electrode for water splitting was a platinum/iridium-oxide-coated titanium flat plate. The two compartments were separated by a cation exchange membrane. Two different pre-treatments were conducted on the granules: (i) acid/base washing followed by degassing, or (ii) only degassing. The acid/base washing was applied to investigate whether potentially present (metallic) impurities residing in the macro- or micropores of AC granules might have an influence on the capacitance. The granules were acid/base washed by submerging them in a $\mathrm{HCl} 37 \%$ solution, placing them on a shaker plate for $1 \mathrm{~h}$ with a speed of $140 \mathrm{rpm}$. Granules were then rinsed through a $315 \mu \mathrm{m}$ sieve with milliQ, resubmersed in milliQ and placed on a shaker plate for $55 \mathrm{~h}$. This process was repeated with a base wash, using a $1 \mathrm{M} \mathrm{NaOH}$ solution, with initial shaking taking $24 \mathrm{~h}$ instead of $1 \mathrm{~h}$. After all rinsing steps had been completed, any remaining air bubbles from the pores were extracted by submerging the granules in milliQ, placing them in an vacuum for 1 hour, while slowly increasing the vacuum pump power to prevent damage to the granules. The granules of the second cell were not treated by acid-base wash, and were only exposed to the air extraction step. The total dry weight of the AC granules in each compartment was determined after concluding the tests, by washing them $3 \mathrm{x}$ with milliQ, then filtering with filter paper and drying in an oven at $105{ }^{\circ} \mathrm{C}$ for \pm 24 hours. All compartments of the two cell were filled with electrolyte (see main text for composition) and placed on a frame, with the capacitive compartment connected to an $\mathrm{Ag} / \mathrm{AgCl}$ reference electrode (Prosense, Oosterhout, The Netherlands; +0.203 V vs SHE).

Electrochemical characterizations of the capacitive electrodes were conducted with a potentiostat (multichannel N-stat DC, Ivium Technologies, Eindhoven, The Netherlands) using three-electrode configuration: the titanium current collector of the capacitive compartment functioned as working electrode, the $\mathrm{Pt} / \mathrm{Ir}$-oxide plate as counter electrode and the $\mathrm{Ag} / \mathrm{AgCl}$ probe as reference electrode.

Capacitive electrodes were "charged" at $+5 \mathrm{~mA}$ for 16 hours or until the potential exceeded $+600 \mathrm{mV}$, after which the potentiostat switched to 8 hours 
of "discharging" at $-10 \mathrm{~mA}$ or until the potential dropped below $-300 \mathrm{mV}$. This cycle was repeated 17 times. During the experiments the potential was measured and using the results the capacitance of the two pre-treatments were calculated and compared.

\subsubsection{Results and Discussion.}

Chronopotentiometric and amperometric plots of the two cells is displayed in Figure S1A. Though the unwashed granules displayed a larger drift in potential during all compared cycles, this was compensated by a reduced packing size per gram as it occurred with the washed granules, and specific capacitance for both unwashed and treated granules were similar, as can be inferred from Figures S1B, C and D. The reduced packing size for granules after acid/base treatment was likely caused by the followed procedure for acid/base treatment, including the use of a stirring plate, which damages/erodes the morphology of the granules allowing for more efficient packing. After drying and weighing the contents of the two cells, there appeared to be $11.0 \mathrm{~g}$ of granules in the cell with treated granules, while the unwashed granule bed only contained $7.4 \mathrm{~g}$. Although reduced packing may turn out benificial for system optimization, to reduce preparation time of follow-up experiments, unwashed granules were used for the CMRB experiment. 

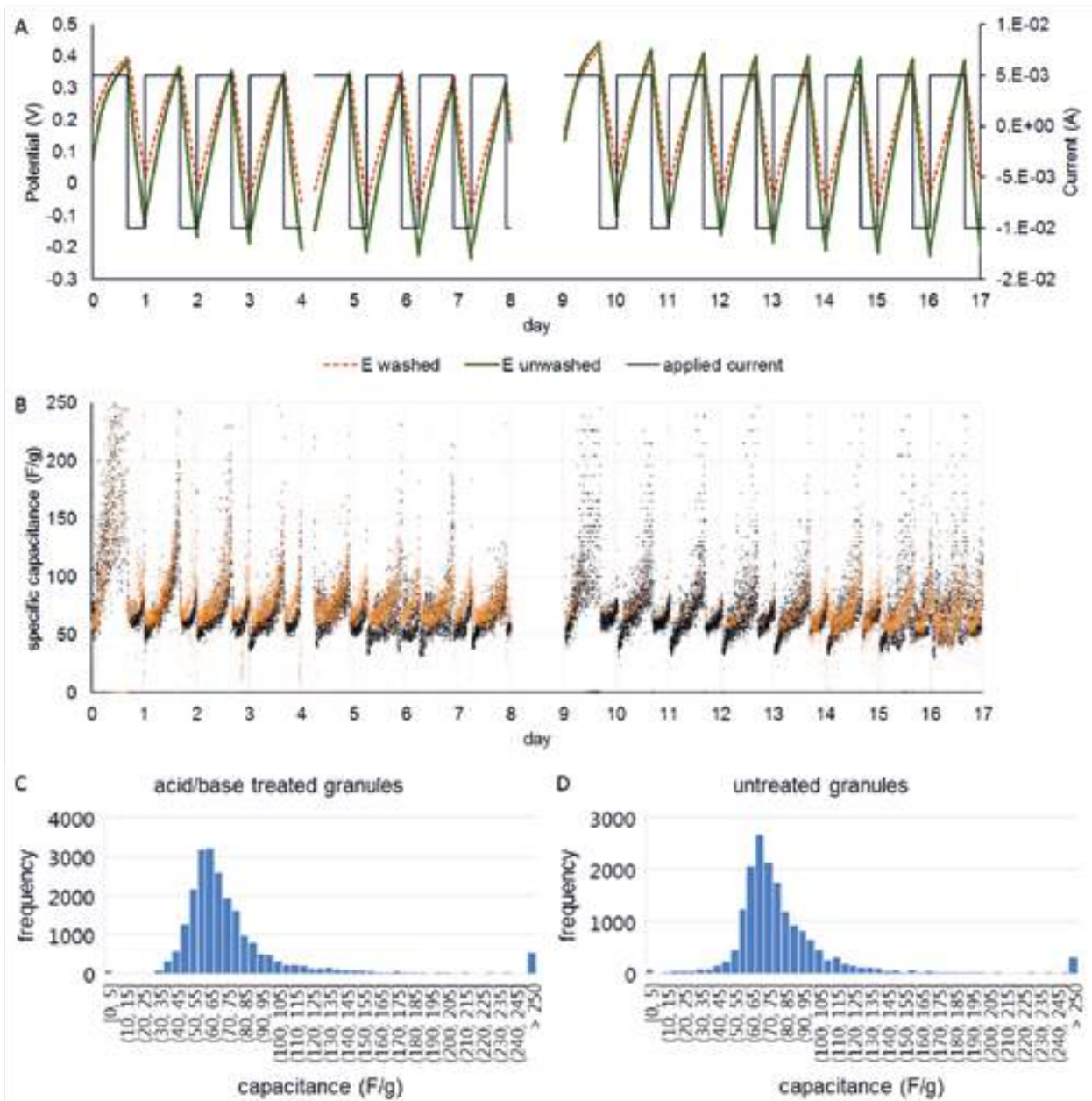

FIGURE S1 | (A) Potentials ( $E$ washed for the acid/base treated granules, $E$ unwashed for the untreated granules) and applied current as recorded during 17 cycles of capacitive counter electrode characterization; (B) specific capacitance as calculated for each measurement interval, based on post-experimental granule weighing, showing large overlap of recorded capacitance for both untreated (orange) as acid/based washed (black) granules, indicating no substantial effect of acid/base treatment; (C\&D) histograms of specific capacitance for both untreated and acid/base treated granules, clearly indicating nominal capacitance of 60-70 F/g regardless pre-treatment. Data captured during day 8-9 was lost due to potentiostat software malfunctioning. 


\subsubsection{DNA extraction methods and 16S rRNA sequencing}

\subsubsection{DNA extraction.}

Frozen graphite felt from bioelectrodes were homogenized using a high speed blender (600-watt motor) for about 5 min (at $45 \mathrm{~s}$ intervals, $15 \mathrm{~s}$ rest) until no large chunks were visible. $0.25 \mathrm{~g}$ of homogenized felt was added to the glass bead tube included in the PowerSoil extraction kit, followed by vortexing for $2 \mathrm{~min}$. The remaining steps of the extraction were performed according to the standard PowerSoil DNA isolation protocol. Extractions were carried out in quadruplicate. Extracted DNA was quantified using the Quantus fluorometer (Promega) and brought to a template concentration of $5 \mathrm{ng} / \mu \mathrm{L}$. After successful extraction had been accomplished, extracts were recombined and put on array in technical duplicates for sequencing.

\subsubsection{16S rRNA sequencing and data processing.}

PCR and subsequent sequencing was done according to the methods described in Takahashi et al $^{41}$. The 16S-based metagenomics analyses were performed using QIIME $^{42}$ version 1.9.1. The primer sequence were removed from the raw sequences reads and then place in a single fasta file using the add_qiime_labels.py script with the options 'cutadapt -m $100-\mathrm{u} 17-\mathrm{u}-21$ '. OTU picking was performed with the script pick_open_reference_otus.py using the SILVA version $128^{43} 16$ S reference database and uclust ${ }^{44}$. The RDP classifier (version 2.2$)^{45}$ was trained with the same SILVA reference database and subsequently used to classify the OTUs. The QIIME script core_diversity_analyses.py was used to calculate alpha- and beta-diversity statistics of the samples. 


\subsubsection{Duplicate plots for Coulombic and energy efficiency}
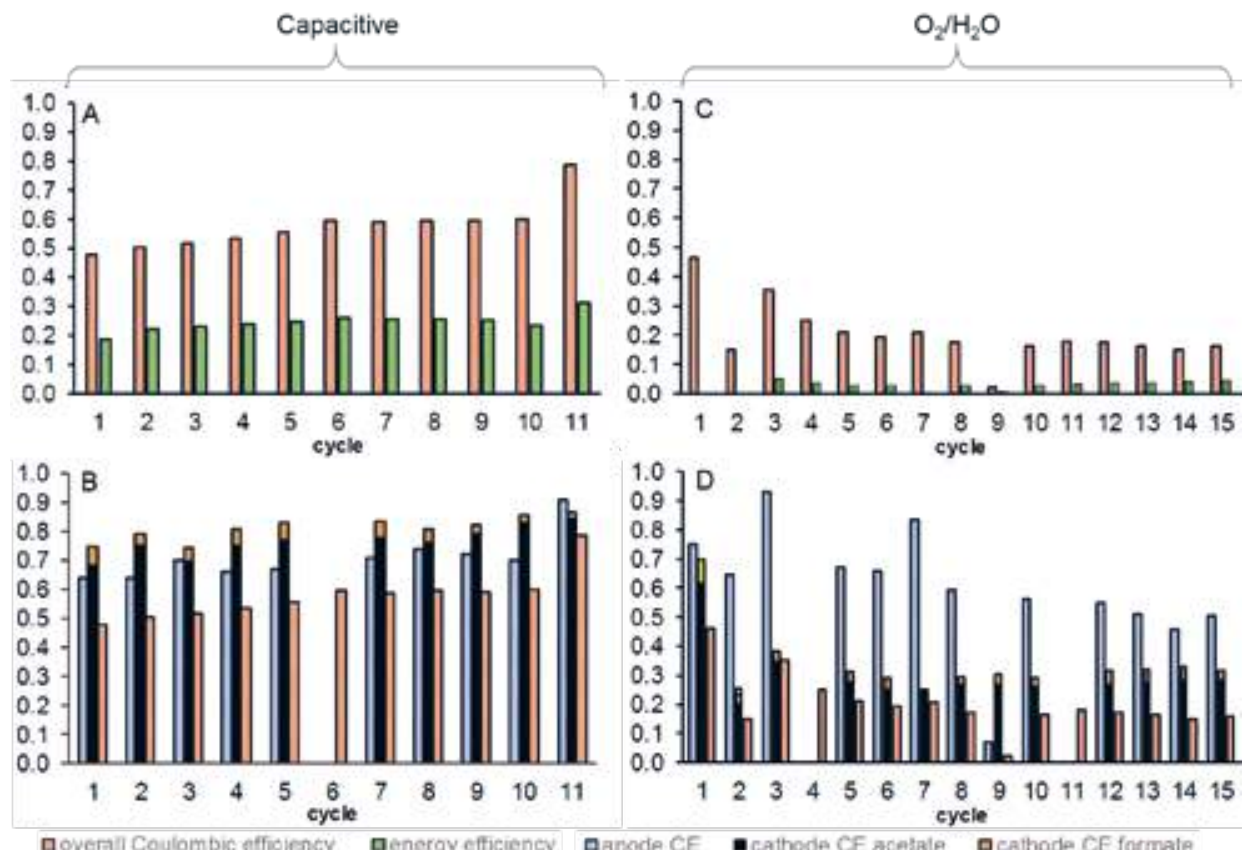

口overall Coulombic efficiency aenergy efficiency aanode CE

- cathode CE acetale a cathode CE formate

FIGURE S2 | Bar plots of the obtained cycle efficiencies for duplicate runs of the capacitive (left, A,B) and oxygen/water redox counter electrode equipped (right, C,D) systems. Upper graphs depict the overall energy efficiency and Coulombic efficiency, while lower graphs show specific anodic and cathodic Coulombic efficiencies, with cathodic efficiency specified per VFA (formate, acetate). During certain cycles no VFA data were available (namely cycle 6 for the capacitive, and cycles 4 and 11 for $\mathrm{O}_{2} / \mathrm{H}_{2} \mathrm{O}$ counter electrode) and thus only overall Coulombic efficiency is reported for these cycles. During the first cycle of the $\mathrm{O}_{2} / \mathrm{H}_{2} \mathrm{O}$ experiments, currents were too high for the counter electrode to sustain positive cell voltage, resulting in a negative EE. At the start of the second cycle for the $\mathrm{O}_{2} / \mathrm{H}_{2} \mathrm{O}$ experiment there was still acetate left-over from the pre-treatment dose administered, and thus no EE is reported for this cycle. At the onset of discharging during the ninth cycle, the potentiostat switched to potential control immediately due to slow response characteristics of the biofilm. This resulted in higher discharge currents and accompanying negative cell voltages, leading to negative EE for this cycle. Prematurely switching of the potentiostat was prevented from cycle 10 onwards. 


\subsection{4 $\mathrm{O}_{2} / \mathrm{H}_{2} \mathrm{O}$ counter electrode characterization}

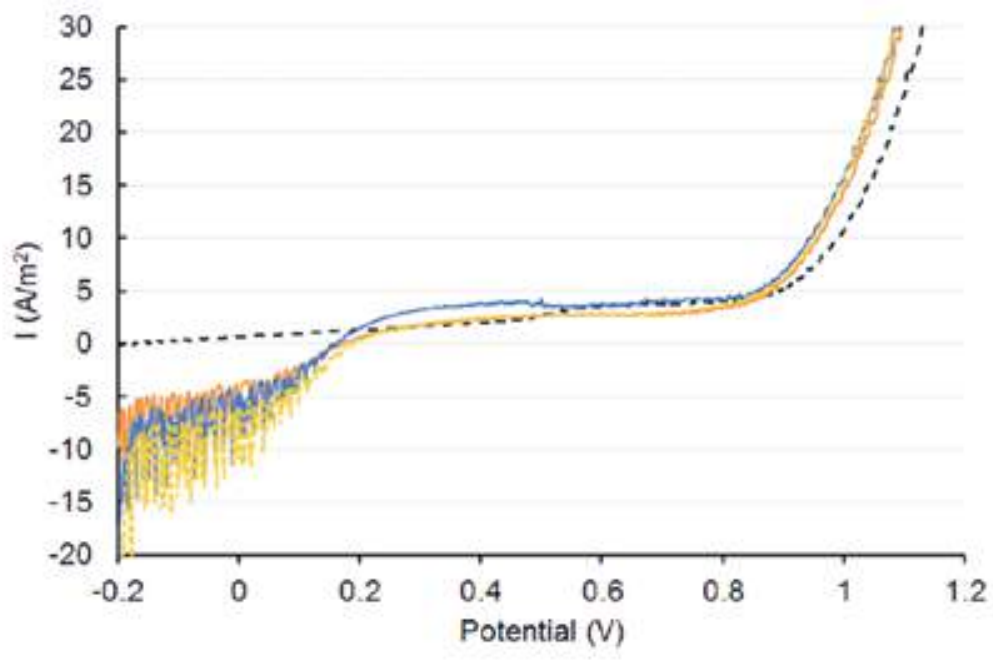

$=-0.3 \mathrm{mg} / \mathrm{L}-4.4-7.3 \mathrm{mg} / \mathrm{L}-14.7-17.95 \mathrm{mg} / \mathrm{L}=15.8-18 \mathrm{mg} / \mathrm{L}$

FIGURE S3 | Polarization plots of the $\mathrm{O}_{2} / \mathrm{H}_{2} \mathrm{O}$ counter electrode at different $\mathrm{O}_{2}$ levels. Prior to the MRB experiments, the $\mathrm{Pt} / \mathrm{IrO}_{2}$ coated titanium electrodes were characterized by performing linear sweep voltammetry (LSV, scan rate $0.01 \mathrm{~V} / \mathrm{s}$, start at negative potential) in the to-be-used electrolyte, but with varying flowrates of administered oxygen supply to the electrolyte. The concentrations indicated in the legend were measured at the start and end of a LSV, respectively. Based on the obtained curves, a flow rate of oxygen of $0.6 \mathrm{~mL} / \mathrm{min}$ was used, which corresponds oxygen concentrations of $4.4-7.3 \mathrm{mg} / \mathrm{L}$. 


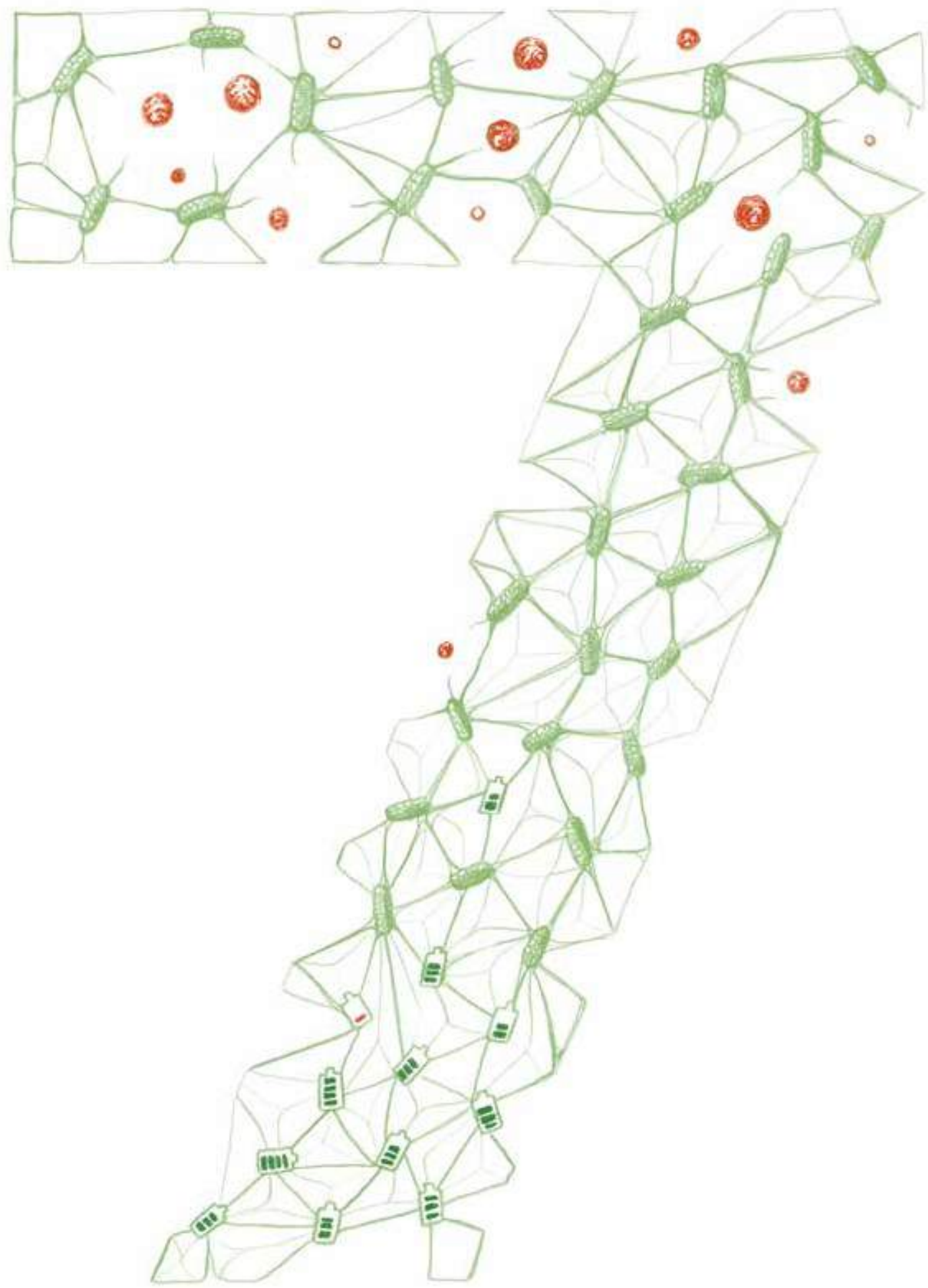




\section{GENERAL DISCUSSION}

Based on the previous chapters, the current status of the microbial rechargeable battery as a future grid energy storage technology is discussed. An overview will be given of the technological hurdles to be taken before the MRB can be successfully scaled up. Based on the disquisition made in the general introduction about the interaction between energy mismatch and feasible ESTs addressing these, a niche market is identified for the MRB. It will be substantiated that, by allowing grassroots implementation of deep decarbonization, the MRB may facilitate the energy transition towards renewable energy sources. 


\subsection{MRB: CURRENT STATUS}

The work presented in Chapter 2 of this thesis described the pioneering work on integrating the carbon dioxide/acetate redox couple in a rechargeable (bidirectional) electrochemical cell. As was shown in the proof-of-concept, it is possible to combine both electrogenic acetate-oxidizing and electrotrophic acetateproducing biota in one system, sharing electrolyte, allowing metabolic activity intermittently for over 15 day-cycles. Despite these successful observations, the continued work on this integrated system as presented in Chapter $\mathbf{6}$ indicated that the counter electrode chemistry used in this proof of concept, ferricyanide, does not qualify as it requires alkaline conditions for stability during practical current densities. This requirement for moderate to high $\mathrm{pH}$ in case ferricyanide is used on the cathode, does not match well with the use of bicarbonate in the anode, as this component tends to cross-over to the catholyte in the form of carbonic acid or gaseous $\mathrm{CO}_{2}$, neutralizing the catholyte $\mathrm{pH}$.

To explore other possibilities for the counter electrode, in Chapter 6 two alternative cathodes were tested, but these showed inferior qualities on all experimental aspects, and pose fundamental challenges for optimization. The use of an oxygen/water counter electrode was accompanied by high overpotentials and dramatically lowered CEs. As a possible strategy for avoiding the large overpotentials typically encountered when using noble catalysts at neutral $\mathrm{pH}$ for oxygen reduction, the future use of ORR biocathodes was mentioned. Indeed, preliminary research on oxygen reducing biocathodes have shown these systems to potentially outperform platina electrodes under these "biological" conditions. However, studies done so far on the stability of oxygen reducing biocathodes do not provide an optimistic outlook, with critical kinetics of dissolved oxygen typically leading to large fluctuations in internal resistances ${ }^{[1,2]}$. For the oxygen evolution reaction, no biologically catalyzed system is known up to date, and performing OER abiotically requires dimensionally stable and oxidation-resistant anodes, usually requiring either high alkalinity (as is the case for nickel anodes) or precious noble metal catalysts ${ }^{[3]}$. Apart from the difficulties encountered at the electrodes using the oxygen/water redox couple, the use of oxygen in combination with the carbon dioxide/acetate electrode brings about the additional challenge of keeping carbon dioxide and oxygen separated. Both being volatile and noncharged, these compounds diffuse relatively easily from anode to cathode and vice versa, lowering CE substantially (in the case of oxygen cross-over) and causing long term stability issues by increased biomass growth and substrate depletion on the side of the anode.

Alternatively, the explored option of using a solid and stationary capacitive counter electrode (Chapter 6), although having appreciable electrode kinetics, had the disadvantages of low charge density (leading to low energy density), and 
a variable electrode potential (requiring more peripheral electronics). Moreover, due to the stationary application of the used activated carbon granules, the tested design lacked the uncoupling of power density and energy density at the side of the counter electrode, normally a specific advantage flow batteries have over other storage technologies.

In Chapter 6 it was suggested a suitable cathode has still to be found, before a MRB might find a suitable application, making a detailed discussion on a specific full electrochemical cell premature. Nevertheless, using water as solvent for electrolyte materials and due to its organic nature, the microbially catalyzed carbon dioxide/acetate redox couple represents a promising anode for future implementation in Aqueous Organic Redox Flow Batteries (AORFB). Therefore, focusing on this biological half-cell, its technical specifications in terms of (i) charge density, (ii) current density, (iii) coulombic efficiency, (iv) (long term) stability and (v) sustainability, will be discussed, and outlooks provided. Moreover, the impracticalities encountered so far with tested counter electrodes underline the importance of accounting for charge transport and separation of compounds between anode and cathode in system design, and guidelines to this end are provided.

\subsubsection{Charge density of the bidirectional carbon dioxide/ acetate bioelectrode}

Achieving a high charge density, in combination with high cell voltage, is crucial for realizing a high energy density. As was reported in Chapter 6, aiming predominantly for achieving high energy efficiency, the experimental part of this thesis did not explore the practical boundaries regarding charge density. For this reason, tested charge densities did not typically exceed $1 \mathrm{MC} \cdot \mathrm{m}^{-3}$, or $0.27 \mathrm{Ah} \cdot \mathrm{L}^{-1}$ (Chapter 2). It thus holds interest to discuss further what kind of charge densities may be achieved for this system based on reported concentrations in the field of MESs and MFCs.

Regarding the cathodic functioning of the bioelectrode, as it is applied in MES, the highest achieved in situ (i.e. as measured in the catholyte) concentration of produced acetate currently reported is by Jourdin et al., who reached $11 \mathrm{~g} \cdot \mathrm{L}^{-1}$ $(186 \mathrm{mM})^{[4]}$. In their study, this was achieved by intermittently adding $\mathrm{NaHCO}_{3}$ and $\mathrm{HCl}$ (so effectively adding both $\mathrm{NaCl}$ and $\mathrm{CO}_{2}$ ) to the catholyte (fed batch). This way, $\mathrm{pH}$ was controlled while preventing - throughout most of the feeding intervals substrate limitation. In a comparable but earlier study, Marshall et al., attained very similar concentrations $\left(10.5 \mathrm{~g} \cdot \mathrm{L}^{-1}\right)$ by continuously sparging the catholyte with $\mathrm{CO}_{2}{ }^{[5]}$. While considering these methods, one could extrapolate that when reversing the process to oxidize acetate, it will require removal of $\mathrm{CO}_{2} /(\mathrm{bi})$ carbonates either 
by gas stripping or salt precipitation in order to maintain $\mathrm{pH}$ neutrality and/or avoid product inhibition (see also section, "MRB integrated design considerations").

Although the concentrations obtained in both studies were similar, it is unlikely that these represent maximally attainable concentrations. Both studies explicitly aimed at achieving high conversion rates and reported achieved acetate accumulation merely for the sake of completeness. However, regarding acetate concentration no steady state was reached and both studies conclude that higher concentrations are likely to be possible, pointing to results obtained in classical fermentation studies, where the production of acetate by a pure culture of Acetobacter Woodii was observed to reach final concentrations of $51 \mathrm{~g} \cdot \mathrm{L}^{-1}$ over the course of less than 4 days, and under atmospheric pressure ${ }^{[6,7]}$. Alternatively to finding microbial strains and conditions allowing for high in situ production of acetate, integrated reactor design may allow for product accumulation by membrane separation. In a study by Gildemyn et al., a cell design was presented in which acetate from an acetate producing biocathode was accumulated by extracting it over an anion exchange membrane up to concentrations of $225 \mathrm{mM}^{[8]}$. Using such an approach could possibly avoid VFA toxicity effects on the biofilm (if present at all).

Presuming that acetate concentrations as high as reported by Straub et al. ${ }^{[6]}$ can be established, either by an electrotrophic microbiome similarly able to tolerate the accompanying (shift in) salinity as reported by Straub et al. ${ }^{[6]}$, or by integrated system design as illustrated by Gildemyn et al. ${ }^{[8]}$, this would convert to a maximum charge density for the carbon dioxide/acetate bioelectrode of $185 \mathrm{Ah} \cdot \mathrm{L}^{-1}$ (under the assumption of $100 \% \mathrm{CE}$ ). Within the field of AORFBs, this would represent an extraordinary high figure; most redox compounds considered for use in AORFBs facilitate only one electron transfer, contrary to the 8 electrons transferred when acetate is oxidized to carbon dioxide, and with maximum solubilities typically in the range of 2-4 M, charge densities thus range from 50 to $100 \mathrm{Ah} / \mathrm{L}^{[9]}$.

The charge density as established by the biocathode is only effective when the acetate formed can be fully oxidized during bioanodic operation. In this aspect the tolerance of anodic biofilm towards high acetate concentrations bears less importance, as with proper substrate loading rate this can be controlled. Especially when a "split electrode" configuration as described in chapters $\mathbf{2}$ and $\mathbf{6}$ is used (i.e. bioanode and biocathode on separate current collectors), or in case acetate accumulation takes place elsewhere (as in the case of the study by Gildemyn et al. ${ }^{[8]}$ ) the bioanode may not even be exposed to high acetate concentrations throughout its entire operation cycle. More importantly, the bioanode should be able to oxidize available substrates as completely as possible, e.g. depleting substrates to sufficiently low concentrations. Fortunately, acetate oxidizing bioanodes have been shown to have high affinity towards their substrate, effectively oxidizing substrates to millimolar concentrations without substantial losses in conversion rates or rising overpotentials ${ }^{[10]}$. 
Potentially further extending the boundaries of charge density may serve the observation by Marshall et al. ${ }^{[5]}$, who reported longer chain carboxylates and alcohols to become more prominent as higher concentrations of acetate were reached, a result which has repeatedly been reported since then also by others ${ }^{[11-14]}$. Although not advantageous in terms of energy efficiency, this would potentially further extend the charge density as more and more reduced carbohydrates are formed (e.g. with 24 electrons stored per molecule glucose versus the previously mentioned 8 of acetate), albeit under the condition they may be efficiently oxidized upon discharging.

\subsubsection{Reaction kinetics of the bidirectional carbon dioxide/ acetate bioelectrode}

In order for the electrode to be practically applicable (i.e. attain high power densities), it will also require fast reaction kinetics to obtain high currents at low overpotentials. As was mentioned in the introduction, various entropygenerating phenomena are in place, in the case of BESs, operating against this desire. Most notably these include (i) electron transfer losses as electrons are transferred between microbe and electrode, (ii) losses occurring within the microbial cell due to metabolic activity / as electrons travel down the electron transfer chain, (iii) (diffusive) charge transfer losses occurring in electrolyte and membrane and (iv) Coulombic losses as undesired processes take place (e.g. hydrogen recycling or oxygen cross-over). Careful and precise characterization of each of these phenomena as they occur in acetate-oxidizing bioanodes and $\mathrm{CO}_{2}$-reducing biocathodes would ideally allow to make robust predictions about and optimization of obtained current densities and overpotentials, provided welldefined system parameters.

Within classical electrochemistry, a convenient amount of potentiodynamic methods to characterize the phenomenological constants involved with reaction kinetics may be found, ranging from as simple as chronoamperometry and cyclic voltammetry to more sophisticated methods like electrochemical impedance spectroscopy (EIS), each having specific strengths, weaknesses and experimental requirements. Illustrative to this end is the well-known experimental design often used when characterizing electrode kinetics in detail, where rotating-(multi) ring disk electrodes, are applied to very elegantly elucidate chemical reaction mechanisms and rates while accurately controlling for mass transfer properties.

Thus, given the common practice of well-controlled and detailed observations done on abiotic systems, when trying to provide an outlook regarding reaction kinetics for the biological carbon dioxide/acetate system, one would initially try to seek for comparable studies applied to BESs. However, although in recent years the application of EIS in bioelectrochemistry has received increased efforts ${ }^{[15,16]}$, 
compared to the level of determinism reached in "abiotic" electrochemistry, the current level of understanding regarding reaction kinetics and their relation to key operational parameters in BESs may be termed preliminary.

There are a number of good reasons for the lack of unambiguous quantitative relationships between reaction rates and operational parameters in BESs. From a practical perspective, the field is relatively young, and the methodology required to perform sophisticated measurements on electrode-respiring bacteria is still under development ${ }^{[17]}$. Moreover, what is making these developments particularly tricky are the relatively long preparations of experiments typical to BESs as biofilms have to develop and require testing on new electrode materials. Growing a homogenous biofilm on a rotating ring disk electrode for instance is something the author hasn't been able to find in literature to be experimented with so far (June 2018).

In addition to these practical challenges, the higher complexity of BESs as compared to non-biological systems requires more extensive modelling, and thus more validation data (and more time): electrolytes used in BESs are multicomponent mixtures featuring, aside from a considerable number of macronutrients, a broad range of metallic and organometallic compounds as co-factors for sustaining microbial growth. Intrinsic to and depending on the presence of (specific) living organisms, the range of possible reactions occurring between these components by workings of enzymatic catalysis expands to more or less anything that is thermodynamically feasible. Furthermore, BESs are far from homogeneous systems, featuring spatially highly structured and (especially using open culture inoculum) diverse biofilms (and potentially also interacting with planktonic cells), which may exhibit unique and specific interactions with (and may even actively alter) specific electrode surfaces. As a result, the additional degrees of freedom the complexity intrinsic to living bacteria introduces both on a molecular level as on higher spatial domains, renders well-established models for electrode reactions (like the Butler-Volmer and Cottrell equation) of limited applicability to BESs. Therefore, quantitative estimates based on these and other "abiotic" models should be regarded more as gross outcomes of specific reactor design and operation, rather than pinpointing intrinsic reaction properties.

What is most troublesome regarding model parameter estimates from studies applying conventional electrochemical models to the domain of bioelectrochemistry, is their validity, and thus predictive power, over time. Apart from non-reversible processes, in non-biotic systems, due to concentration polarization, fluctuations in performance are expected to occur on relatively short time scales, resulting in typically short characterization experiments. However, as biofilms are known to be adaptive towards changes in growth conditions, this extends the relevant time scale on which performance has to be characterized to a scale of potentially multiple generations of cell division. Failure to account for 
this may lead to contradicting findings in literature, and an outstanding example forms the ambiguity regarding the effect of polarization on current density and cell growth ${ }^{[18-21]}$.

With above difficulties and reservations serving as a disclaimer, an assessment of gross reaction rates as they can be achieved by bioanodes and biocathodes can be made on basis of the numerous (chronoamperometric) studies already done on these systems. In doing so, corrections, conversions and/or a selection may have to be applied, so that values are at least "standardized" or reported at similar or representative conditions.

A pragmatic but nevertheless thorough and very recent assessment of literature aiming to make this comparison of electrode kinetics in both MFC anodes and MES cathodes has been recently covered in the works of most notably Baudler et al., Guo et al., Kerzenmacher, Patil et al. and Jourdin and Strik ${ }^{[22-25]}$. Although these studies also explicitly state important reservations related to estimating achievable current densities in bioelectrodes, an order of magnitude estimation from these works seems possible. Focusing on studies with nominal overpotentials of typically 0.2 to $0.3 \mathrm{~V}$ for the anode reaction, stable current densities of 15 to $25 \mathrm{~A} \cdot \mathrm{m}^{-2}$ may be achieved, when results are adequately converted to be expressed towards specific surface area. Conversely for the cathode reaction, by applying considerably higher overpotentials of 0.3 to $0.5 \mathrm{~V}$, current densities between 5 and $10 \mathrm{~A} \cdot \mathrm{m}^{-2}$ have been reported ${ }^{[26]}$.

Regarding the typical overpotentials mentioned in literature, their relative impact on full-system energy efficiency will strongly depend on the choice of counter electrode, and thus, the resulting operational cell voltages. As a short illustration, the resulting VE using two hypothetical counter electrodes are compared in Table 1.

As Table 1 shows, the overpotentials mentioned result in considerable VE losses. Especially the larger overpotentials as occurring at the biocathode for acetate production contribute to this loss. As was explained in more detail in chapter 6 of this thesis, and further described in the review by Jourdin and Strik $^{[26]}$, these higher overpotentials commonly observed for the cathodic reaction

TABLE 1 | Projected attainable voltage efficiencies for two hypothetical counter electrodes, assuming practical overpotentials at the carbon dioxide/acetate anode. The outcome clearly shows the synergistic effect a higher counter electrode potential has on this performance indicator.

\begin{tabular}{|l|l|l|l|}
\hline $\begin{array}{l}\mathrm{CO}_{2} / \mathrm{C}_{2} \mathrm{H}_{3} \mathrm{O}_{2} \\
\mathrm{E}_{0}=-0.29 \text { vs } \mathrm{SHE}(\mathrm{V})\end{array}$ & $\begin{array}{l}\text { Hypothetical counter } \mathrm{E}_{0}=0.75 \\
\text { or } 0.25 \text { vs } \mathrm{SHE}(\mathrm{V})\end{array}$ & Cell Voltage $(\mathrm{V})$ & Round trip VE \\
\hline $\begin{array}{l}\text { Charging: }-0.59 \\
\text { Discharging: }-0.09\end{array}$ & $\begin{array}{l}\text { Charging: } 0.8 \\
\text { Discharging: } 0.7\end{array}$ & $\begin{array}{l}\text { Charging: } 1.39 \\
\text { Discharging: } 0.79\end{array}$ & 0.57 \\
\hline $\begin{array}{l}\text { Charging: }-0.59 \\
\text { Discharging: }-0.09\end{array}$ & $\begin{array}{l}\text { Charging: } 0.3 \\
\text { Discharging: } 0.2\end{array}$ & $\begin{array}{l}\text { Charging: } 0.89 \\
\text { Discharging: } 0.29\end{array}$ & 0.32 \\
\hline
\end{tabular}


are ascribed to $\mathrm{H}_{2}$ being an intermediate for acetate production. However, recent advances in MES have suggested that tailored composition and metabolic adjustment of the microbial community may improve cathodic electron transfer by making use of direct electron transfer mechanisms ${ }^{[27]}$ and experimental efforts seem well on their way to establish this ${ }^{[28]}$.

In order to maintain the surface specific currents into volumetric current densities, a proper cell and electrode design would be required, avoiding steric hindrance due to clogging and assuring good mass transfer while stacking up multiple layers of electrode/biofilm in a confined space. Best practices so far to this end are obtained using metal foam structures, or reticulated vitreous carbon, showing effective surface-to-volume multiplication factors (active $\mathrm{cm}^{2} \cdot \mathrm{cm}^{-3}$ ) roughly between 4 and 10 . Using the previous ranges given for current density, this translates to 6 to $25 \mathrm{~A} \cdot \mathrm{L}^{-1}$ (or kA.m ${ }^{-3}$ ) for bioanodes, and 2 to $10 \mathrm{~A} \cdot \mathrm{L}^{-1}$ for biocathodes. Assuming a hypothetical counter electrode allowing an operational cell voltage of a convenient $1 \mathrm{~V}$, this leads up to indicative power densities of 6 to $25 \mathrm{~W} \cdot \mathrm{L}^{-1}$ upon discharge. Although certainly not record setting power densities within the broader context of energy storage technologies, such volumetric current densities would be comparable to typical AORFB electrodes ${ }^{[0,29,30]}$.

\subsubsection{Coulombic efficiencies of the bidirectional carbon dioxide/acetate bioelectrode}

Voltage losses imply a direct loss of exergy to heat, and as such lower the energy efficiency of the electrode. Although Coulombic losses not necessarily lead to (excessive) entropy production, they still negatively impact energy efficiency, as electrons are lost to processes which do not interface with the (intended) electrode, and therefore these charges are not retrieved upon discharge. As was explained in chapter 5 and the introduction of this thesis, Coulombic losses in bioelectrodes take place inevitably by means of biomass production, for which the boundaries are set by the available free energy. In practice, lower biomass growth is often obtained than what could be expected based on equilibria calculations, as also microbes are not $100 \%$ efficient in harvesting free energy for growth, and may exhibit changes in growth stoichiometry under conditions where catabolic processes are not rate limiting ${ }^{[31]}$. In case no other electron diverting reactions take place, this results in higher CEs. For saline conditions, CE loss due to biomass formation may be hypothesized to decrease further as more energy is spent on maintenance of the intercellular environment.

High CEs for the anode reaction are often reported in studies where artificial wastewater and electrolytes have been used, in combination with enriched inocula or addition of methanogen inhibiting compounds, ensuring low presence or even total absence of methanogenic bacteria. Under these conditions, and at practical 
anode potentials, CE for anodes ranges typically between 90 and $100 \%$ when acetate is used as substrate ${ }^{[32]}$. For acetate producing cathodes, attaining high CEs towards acetate has shown to be slightly more challenging. Often this is due to the formation of $\mathrm{H}_{2}$, which in case of insufficient scavenging by surrounding biofilm may end up in the headspace as unwanted side-product. Best practices for biocathodes however, as reported in multiple studies, have shown high CEs towards production of acetate, up to nearly $100 \%$, to be feasible ${ }^{[26,33]}$.

Both for biocathodes and for bioanodes, however, considerably lower CEs may be obtained in case methanogens are not being suppressed, or kept absent ${ }^{[32]}$. Suggestions for setting operational parameters in such a way that methanogens are outcompeted, have been extensively provided in chapters 3 and $\mathbf{4}$ of this thesis. However, these suggestions were made within the context of continuous systems in which slow growing biomass can be washed out. This is not the case in the closed system of a battery. Therefore, methanogen-inhibiting chemicals were added to the electrolyte used in the experiments covered in this thesis, and so has been done in numerous other studies, and successfully so far.

As a last consideration regarding potential CE losses, the potential crossover of product and substrates to the counter electrolyte must be mentioned. Apart from hydrogen-evasion in biocathodes, especially the crossover of acetate forms a potential CE loss, as this compound, although mainly present in anionic form at neutral $\mathrm{pH}$, may diffuse relatively easily through membranes in its undissociated form, even when cation exchange membranes are used. From this perspective, it would be a big advantage if a counter electrode is found which has no reactivity towards acetate.

To conclude this section, Coulombic efficiency as reported both for MES cathodes and MFC anodes can achieve nearly $100 \%$, with only small but inevitable losses due to biomass growth. If replicable within the setting of a bidirectional electrode, as it is applied within the MRB, CE losses are not expected to significantly influence the energy efficiency of a single charge/discharge cycle, but they may have impact on the long term stability and energy density of the MRB.

\subsubsection{Long term stability of the bidirectional carbon dioxide/ acetate bioelectrode}

In chapters 2 and 6 of this thesis, experiments with the carbon dioxide/acetate electrode were conducted over periods typically within the range of 15-30 days. To become a relevant technology, the stability of the bioelectrode has to be proven also on a considerably longer timescale. Especially the following aspects therefore should be taken into account: (i) the long term impact of biomass growth, (ii) long term inhibition of methanogenesis and (iii) electrode maintenance. 


\subsubsection{Long term impacts of biomass growth}

Biomass growth rate is not so high that it forms major problems on a per cycle basis, but over the course of hundreds of cycles, its formation and lesser degradation will start to accumulate nutrients and carbon. Ultimately, this will form a problem for further proper functioning of the electrode as nutrients available for microbial growth and activity will be depleted. Moreover the inactive biomass may clog up pores in case porous electrodes are used. The non-degradable part of the biomass that thus will form will require (periodic) removal, and the nutrients assimilated within this inert fraction of the biomass will have to be replaced. Foremost the periodicity/rate with which this has to be carried out, depends on the growth rate of the electrogenic biomass, which in term may be related to the composition of the microbial community (especially the presence of heterotrophic organisms may play a role here) and the overpotentials applied. The use of OCT as described in chapter $\mathbf{5}$ could contribute to determine the necessary periodicity of biomass removal, by providing better insight in the relation between biomass thickness, distribution, dynamics and cell performance. Regarding methods for removal of inactive and non-degradable biomass, either sludge removal by integration of a solids separator could be considered, or the system as a whole would need periodic (chemical and/or mechanical) cleaning, after which (the electrolyte is replaced and) the system is restarted.

\subsubsection{Long term inhibition of methanogenesis}

Neither the formation of methanogenic biomass, nor the formation of methane in biocathodes, contributes to electrode performance and therefore needs to be prevented. Although the bespoken addition of 2-BES for inhibiting methanogens is effective on the short term, it remains questionable, also when applied to the closed system of the MRB, how sustainable this practice turns out to be on longer term. Measurements performed in chapter 2 showed a gradual decline in the concentration of this compound, indicating either degradation or adsorption of it by the present biota. Once this compound would lose its effectiveness (and it has been shown in various reports to be able to do $\mathrm{so}^{[34,35]}$, methane formation may lead to rapid inactivation of the electrode system: the gas is poorly soluble, will evade the system quite easily, and can only very slowly be oxidized with bioanodes. As was explained in more detail in chapter 3 and 4, applying high current densities and optimizing reactor design to minimize diffusion limitations for electrode bound biofilms, might allow to outgrow methanogens on short term. However, almost regardless how long it takes, in a closed system where there is no washout of slow growing species, if methanogens grow (albeit slowly), it will require periodic cleaning to wipe them out. The only other possibility would be to prevent presence of methanogens completely, but this would introduce the additional and impractical criterium of axenic construction and operation of the electrode so that contamination is prevented at all times. 


\subsubsection{Criteria with respect to electrode maintenance}

Provided a proper state-of-charge, conventional electrodes may be stored over long periods of time, after which they may be directly put to work again. For a bioelectrode to work, it depends on metabolically active bacteria to fulfill the electrode reactions. Although in some cases bacteria are renowned for their ability to survive extreme environments and periods of inactivity, for a smooth and efficient bioelectrode functioning it bears importance that the biofilms are kept alive and can swiftly resume metabolic activity at all times. Failure to do so would require (potentially extensive) start-up periods, in which bacteria are either reactivated, or have to be regrown. To prevent this, the bioelectrode will require a minimum intermittency in use. In the experiments reported in chapter 2 and 6 of this thesis, it was shown that biocathodes and bioanodes could be operated intermittently in 16-8 hours intervals, allowing active/non-active periods to alternate, without substantial loss of functionality and efficiency. However, robustness towards longer periods of electrode inactivity would need further assessment.

As a second aspect of electrode maintenance, the robustness of the bioelectrode against overcharging or over-discharging currents (i.e. fluctuations in current density and overoxidation/reduction of the electrolyte) needs to be taken into account. As was explained before, the biofilms of these systems are likely to develop specifically to the conditions occurring, with microbial composition, biofilm structure and thickness being key aspects. Current fluctuations during charging may result in CE loss to hydrogen formation, as the present biota is not capable of sufficiently scavenging the gas for production of acetate, or - in more severe situation - may lead to biofilm ablation, which will require time to recover. In the case of anodic functioning, applying current higher than what the biofilm is accustomed to may lead to adaptive changes in the bacterial ETCs, potentially leading to higher overpotentials and loss in energy efficiency. Moreover, in case currents exceed rates with which electrons can be delivered based on acetate oxidation, this will result in oxidative processes that may damage the biofilm, or permanently damage the electrode (for instance by forming passive layers). It seems that by its nature, the bioelectrode thus would need protection against these conditions occurring, and for a practical application, enhanced load leveling thus would need to be considered.

\subsubsection{Sustainability of the bidirectional carbon dioxide/acetate bioelectrode}

As was mentioned in the introduction regarding sustainability requirements of a future EST, it bears importance to assess the availability of a technology's material requirements in combination with the intended use and scale of that use. Although 
at this stage of development not much can be said about the potential scale of use the bioelectrode would be applied at, some optimistic remarks can be given regarding the materials (and energy) required for its construction and operation.

A first thing to consider is the choice of electrode material. Although an almost overwhelming amount of information can be found in literature on this account, fortunately the work done so far on electrode modifications for application in BESs has been reviewed thoroughly from time to time. As most recent and noteworthy may be mentioned the reviews by Guo et $\mathrm{al}^{[23]}$, Jourdin and Strik ${ }^{[26]}$ and Kerzenmacher ${ }^{[24]}$. Presumably the best summary of the insights gained so far on what an ideal electrode would feature, is provided by the review of Guo et al. ${ }^{[23]}$, describing it to have:

"good biocompatibility, high specific and electroactive surface area, strong chemical stability (includes corrosion resistance), fast electron transfer at microbe-electrode interface; high conductivity, excellent mechanical strength, good processability and scalability, low environmental impact (manufacturing footprint, recyclability) and low cost"

Although in the same publication the authors mention that "neither the carbon-based nor the metal-based materials fulfil these criteria completely", in the later review by Jourdin\&Strik ${ }^{[26]}$, a thorough comparison of studies in terms of specific current densities and used potentials, is made, and identifies as best practices the use of flame oxidized stainless steel felt, carbon-nanotube coated reticulated vitreous carbon, or - albeit only validated on a scale of an individual granule - activated carbon. Altogether, it may be hypothesized that the ideal electrode as described above will be a composite material, consisting of a highly conductive backbone (either metallic or glass-like carbon), a surface area-enhancing peripheral structure on micrometer scale (presumably graphite based), and possibly biocompatibility/electron transfer-improving surface modifications (like carbon nanotubes or functional groups) on a (sub)nanometer scale. It can thus be concluded that with steel and carbon, all necessary materials are available for making the microbial electrode of the future. This would be beneficial in terms of the sustainability of this future electrode, as steel alloys are normally made from abundant metal species, and are highly recyclable. Most carbon structures can be made from either renewable (biomass based) sources or from plastic waste ${ }^{[36]}$.

The second component of the bioelectrode to be considered in terms of its sustainability is the electrolyte. Being aqueous, it already has a marked advantage to this point compared to non-aqueous electrolytes, often requiring petrochemical precursors. Moreover, all chemicals used in the experiments described in this thesis, and in other work on BESs so far, contain chemicals typical for microbial growth, and as such are almost by definition abundant, non-toxic and/or biodegradable. To further illustrate this point, at least one 
study has reported high current density in acetate oxidizing anodes using merely compost extracts as electrolyte, with acetate and table salt added. In contrast to conventional flow batteries, metallic species are only required in trace amounts $(<\mu \mathrm{M})$. As a single exception to this sustainability praise regarding bioelectrolytes, may serve the earlier mentioned use of the methanogeninhibitor 2-BES. This compound, applied in considerable amounts (10mM) in the works described in this thesis, forms the only not-naturally occurring additive of the electrolyte, and its lifetime and effect, both within the reactor as in natural ecosystems, is yet to be investigated.

Concluding this section, although a preliminary assessment may provide an optimistic outlook regarding sustainability of the bioelectrode, further extensive LCA will be required to more thoroughly study its environmental impact.

\subsection{MRB INTEGRATED DESIGN CONSIDERATIONS}

The previous section considered specific performance aspects for the carbon dioxide/acetate electrode, most importantly charge density, Coulombic and voltage efficiency. For each of these performance aspects, an indication of their maximally attainable values was provided based on best practices in literature. The assessment provided attractive perspectives for the electrode on these aspects, showing charge densities and Coulombic efficiencies exceeding those typically encountered within AORFBs to be plausible. Moreover, based on figures obtained from separate biocathode and bioanode experiments, competitive current densities can be achieved without excessive overpotentials / energy losses.

However, as the work presented in chapter 2 and 6 of this thesis also showed, notable differences exist in terms of performance achieved between studies involving continuous systems, optimized for a particular purpose, and a rechargeable battery, in which a closed system is being studied and conversions by definition must be able to be completely reversible (i.e. bi-directional). To this end, it bears importance to consider what it requires in terms of system design to close these gaps.

Regarding charge density, proper $\mathrm{pH}$ control is of utmost importance when high concentrations of acetate need to be reached. The reason for this is twofold. On one hand, the reaction stoichiometry requires 8 protons for each acetate molecule formed. As biofilms are by nature prone to diffusion limitations, it would require a high bulk concentration of protons in order to prevent severe overpotentials by concentration polarization. On the other hand, when such a 
high concentration of (easily) dissociated protons would be realized by means of increased acidity, this would conflict with the requirement of most bacteria for (more or less) neutral pH, especially under (momentary) periods of non-turnover. Set aside the possibility of using extremophilic bacteria that can actually cope with such conditions, these two aspects taken together lead to the conclusion that for obtaining high concentrations while keeping the microbes active/intact, heavy $\mathrm{pH}$ buffering is the way to go. Fortunately, the carbonic acid/bicarbonate buffer already required for forming acetate can fulfil this role, but increasing its concentration will in turn result in higher salinity, which may be posing an additional (and potentially mitigatable ${ }^{[37]}$ ) stress factor for the present biota.

Looking deeper into buffer strength requirements, some practical implications on system design can be foreseen when high charge densities are to be reached. For instance when aiming at a final concentration of $0.8 \mathrm{M}$ acetate in the anode, a total of $1.6 \mathrm{M}$ of inorganic carbon would need to be reduced. At the same time, 8 protons consumed per acetate molecule formed, result in approximately $6.4 \mathrm{M}$ of alkalinity that will need to be neutralized (some weak acid effects of the shift in bicarbonate/acetate speciation set aside). This may be done either by (i) completely buffering this or (ii) having a proton coupled electron transfer (PCET) reaction in the counter electrode.

In case of a PCET counter reaction, the protons required for producing acetate can be sourced from the counter electrode. However, to make use of this possibility, it will be crucial having protons/hydroxyls shuttled between anolyte and catholyte efficiently, without large $\mathrm{pH}$ gradients. This would in turn require a buffer which can crossover between the two electrode compartments in both protonated and deprotonated form, while still allowing selectivity of ion transport (preventing acetate to reach the counter electrode or vice-versa, having the counter reactant ending up at the cathode).

A fit example for such a buffer which can effectively support proton transfer between anode and cathode, is for instance when an anion exchange membrane is used to have bicarbonate going from cathode to anode, combined with gaseous/ uncharged $\mathrm{CO}_{2}$ going back from anode to cathode. This situation is schematically depicted in Figure $\mathbf{S 1}$ in the appendix, and also in Figure $\mathbf{1}$ here to allow for direct inspection.

When this concept would be applied to the carbon dioxide/acetate electrode, a next point of attention would be the Coulombic efficiency: employing an anion exchange membrane, it would either require a counter electrode non-reactive to acetate, or a bicarbonate selective membrane should be used while care should be taken regarding the possible transfer of acetate to the counter electrolyte by gas exchange, although the latter might be a smaller problem as the $\mathrm{pK}_{\mathrm{a}}$ of acetic acid is lower than that of carbonic acid. Similarly, depending on the charge and chemical properties of the counter electrode reactant, this may also crossover to the anolyte, and appropriate measures should be taken likewise. 


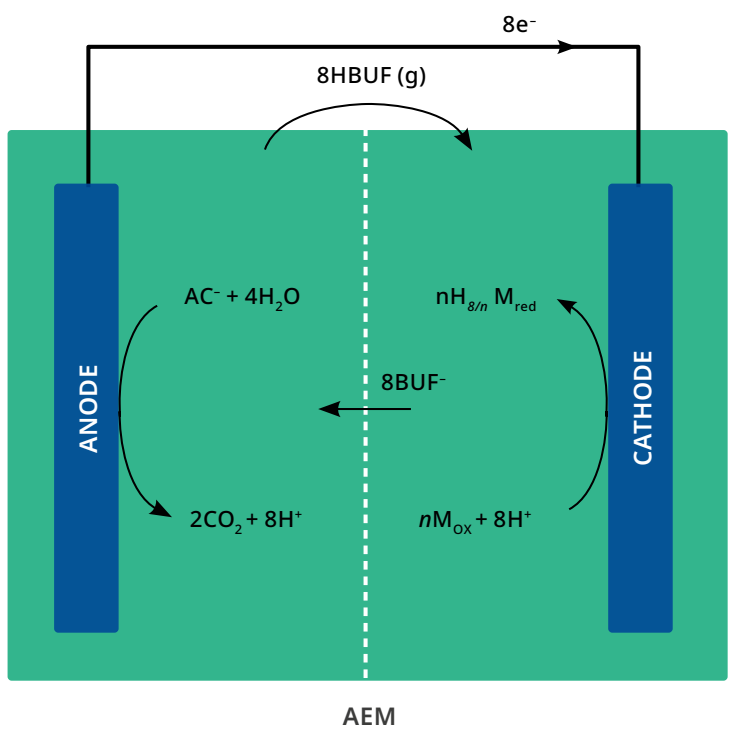

FIGURE 1 | Schematic of a (bio)electrochemical system in which both anode and cathode reactions are proton-coupled. A minimal pH gradient may be achieved when an anion exchange membrane is applied in combination with a volatile buffering compound (e.g. carbon dioxide/bicarbonate) which can effectively shuttle protons as indicated between the two half cells

When the carbon dioxide/acetate electrode is combined with a non-PCET counter reaction, there seems to be no other option left than to neutralize the alkalinity/acidity as it occurs during charge/discharge cycling by addition of a buffer. An important design choice in this case may be whether a non-volatile, or volatile buffer is applied. The latter has the advantage that it can easily be removed again upon later oxidation of the acetate, and the carbonic acid $/ \mathrm{CO}_{2}$ system seems suited to this end as well. Nevertheless, it may be no surprise that this would result in extremely high salinity, progressively established as the process takes place. Where in the system salinity would accumulate depends mainly on membrane choice, as is depicted most clearly when comparing Figure S9 and Figure S11 in the Appendix, which are printed here as Figure 2 and $\mathbf{3}$ for convenience.

Concluding the considerations above, three foremost design choices can be identified which have major impact on the most defining performance aspects, as they to large extent define the mass transfer characteristics of the system:

1. Whether the counter reaction is proton coupled (PCET) or not (NPCET)

2. Whether a volatile or non-volatile buffering compound is used

3. Membrane transfer specificity 


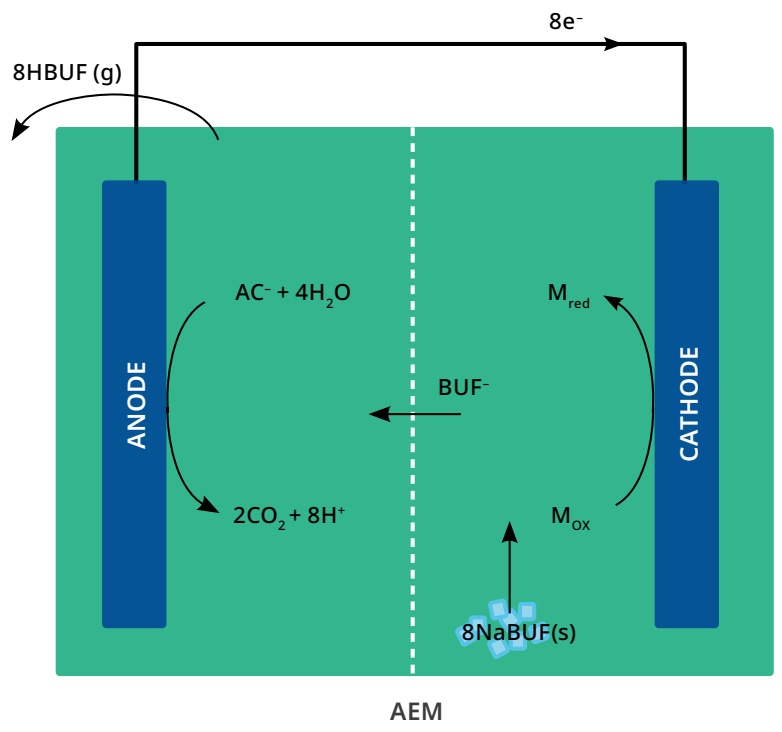

FIGURE 2 | Shows a schematic of a (bio)electrochemical system in which a proton coupled anode reaction is combined with a non-proton coupled cathode reaction and with use of an anion exchange membrane. In order to minimize pH fluctuation by acidification at the anode, a buffering compound has to be transported over the membrane from cathode to anode. Assuming high concentration of acetate to be achieved, required amounts of buffer will exceed solubility constants and thus precipitation of buffer salts will occur at some point throughout the charging/discharging cycle. Using a AEM, this is most likely to occur at the cathode side, as this will be the place where anions and cations both accumulate. In case a volatile buffer system is used, salinity fluctuation can be largely prevented on the anode side by pressure regulation (indicated by the removal of gaseous buffer on the top left).

In designing a MRB to obtain a balanced performance on discussed technical aspects, an integrated approach is required in which the combined effects of these three design choices are considered. The many practical uncertainties involved make this a complex task, and to that end a long road of iterative experiments presumably still lies ahead. For a more extensive exploration of possible cell designs based upon above three design choices and their foreseen pros and cons, the reader is referred to the appendix of this chapter. 


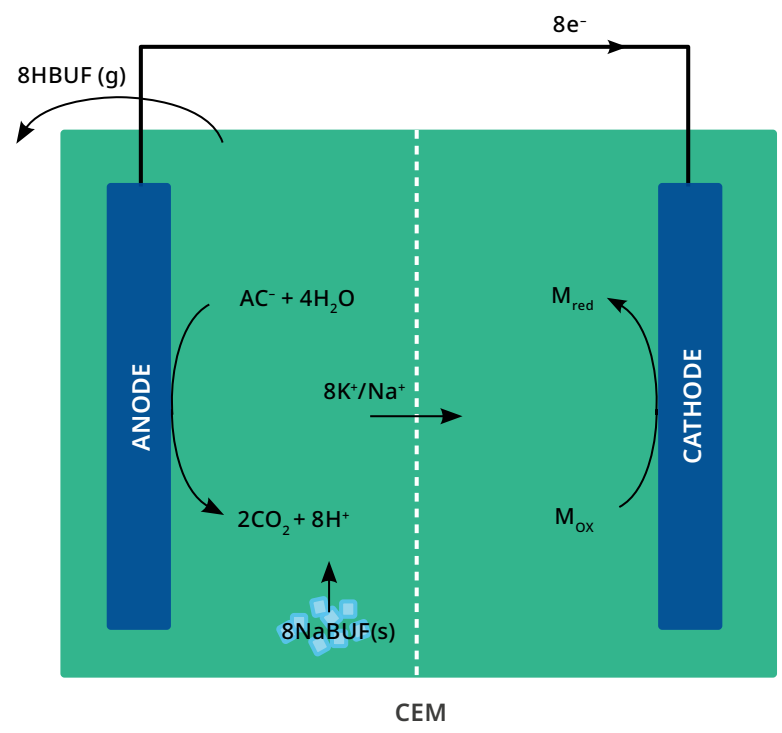

FIGURE 3 | Schematic comparable to figure 3/S9, but now with a cation exchange membrane, which may be required to prevent anionic substrate cross-over causing coulombic efficiency losses. Notable difference with Figure S9 regards the salt deposit location, which is now located at the anode side.

\subsection{FUTURE OUTLOOK OF THE BIDIRECTIONAL CARBON DIOXIDE/ ACETATE BIOELECTRODE}

As the experimental work, developed theory and technical aspects as discussed above for the bidirectional carbon dioxide/acetate electrode have shown, this electrode possesses relevant properties making it a potentially attractive electrode for integration in AORFBs. However, before reaching this phase, a number of key challenges will have to be faced first. Based on the limitations and knowledge gaps as identified in this thesis, the most important points to address are:

- Optimization of charge density by changing system design and operation, allowing $\mathrm{CO}_{2}$ to be used both as reactant and as buffering agent, while preventing acetate to be removed by stripping / cross-over. Especially in case a non-proton bound counter electrode reaction is used, the potential use of halotolerant microbiomes for achieving higher concentrations at increased salinity must be explored.

- Optimization of electrode kinetics and alleviation of mass transfer limitations by improving electrode design in combination with a suitable 
microbial community composition. Concrete recommendations and design considerations regarding bioelectrodes have been provided in literature ${ }^{[23,24,26]}$, while promising results with lowered overpotentials for the cathodic reaction have been achieved recently by microbial culture enrichment using inversed polarity treatments ${ }^{[38]}$. A combined approach applying these best practices both in electrode materials and morphology, and established microbial community composition, may yield synergistic results.

- The impact of key operational parameters on long term stability and performance of the system should be studied by means of a thorough characterization of the obtained kinetics, mass balancing and microbial community analysis. Special attention should be paid to long term effectiveness and biological fate of methanogen inhibitor 2-BES.

Once above key challenges have been properly addressed, the search may start for finding the match regarding a proper counter electrode, so that a real AORFB is formed. In line with the requirements set for the carbon dioxide/acetate electrode, the system compatibility requirements this alternative counter electrode would have to comply with are:

- High concentration / charge density

- High potential

- Good kinetics

- Highly stable, with high CEs, with either the possibility to avoid crossover of reactants from anode to cathode and vice versa (anion, so that a cation exchange membrane can be used), or not affected by compounds possibly crossing over

- Sustainable and safe

Based on a state-of-the-art review of literature on AORFBs, as promising examples of such electrodes may be mentioned the use of the stable free radical TEMPO, and related metallo-organic derivatives, like Ferrocene ${ }^{[9,30]}$. With achievable concentrations up to $4 \mathrm{M}$, charge densities may be reached of $100 \mathrm{Ah} \cdot \mathrm{L}^{-1}$. Depending on $\mathrm{pH}$ and ionic strength, various potentials may be obtained, but formal potentials as high as $0.6 \mathrm{~V}$ (vs. SHE) are possible, with current densities reported reaching over $50 \mathrm{~A} \cdot \mathrm{m}^{-2}$. In addition to these synthetic compounds, substantial research efforts are done on the use of Quinones for application in AORFBs. Although conventionally deemed unsuitable due to poor solubilities, functionalization of carbon slurry by impregnation with Quinones has led to interesting preliminary results, with charge densities of $50 \mathrm{Ah} \cdot \mathrm{L}^{-1}$ at formal potentials of $0.42 \mathrm{~V}$ (vs. $\mathrm{Ag} /$ $\mathrm{AgCl})$ being possible under neutral conditions ${ }^{[39]}$.

Extrapolating a hypothetical situation in which referred Quinone impregnated slurry would be successfully used for establishing a AORFB in combination with 
the carbon dioxide/acetate electrode, energy densities of approximately $25 \mathrm{Wh} \cdot \mathrm{L}^{-1}$ and power densities of $>10 \mathrm{~W} / \mathrm{L}$ (based on symmetric sizing of anode and cathode and assuming the anode to be current limiting) would be on the horizon.

\subsection{THE POSITION OF ENERGY STORAGE AS SOLUTION OF THE ENERGY MISMATCH, AND THE NEED FOR GRASSROOTS IMPLEMENTATION}

As was elaborated on in the introduction of this thesis, the socioeconomic position of energy storage depends heavily on the size and nature of a present energy mismatch. However, much uncertainty exists about the future size and severity of the energy mismatch as it will take shape during the transition to "deep decarbonization". In general, factors that can either mitigate or propagate mismatch are (i) power supply capacity and characteristics, (ii) interconnection or grid inertia, (iii) demand response and (iv) energy storage.

Looking at the grid challenges analysis for the UK as discussed in the introduction of this thesis, energy storage facilities to be sought after are those that can provide backup within seconds up to several days, thus both serving backup due to clouding effects in solar, gusts and lulls in wind, and daily fluctuations caused by end user demands. The implementation in this intermediate phase is most likely to take place in an incremental manner, with intentional communities, like eco-villages, opting in one by one. The scale of implementation thus ranging between single households and several hundreds of them, this will require typical storage capacities in the range of 10-1000 $\mathrm{kWh}$, for which the footprint will have to fit inside the communities property. Additionally, for communities facing strong seasonal patterns in energy mismatch, the backup duration and capacity would need to be expanded by an order of magnitude, lasting typically for several months with corresponding capacities ranging from 1 to $100 \mathrm{MWh}$, would need be that current (western world household) energy consumption patterns be unaltered.

Looking at currently available technologies, the selection then would narrow down to exclusively electrochemical methods, as they feature a broad range regarding discharge duration and operational power density, while characterized by low rates of self-discharge. For mitigating daily mismatches, conventional examples would be lead-acid or LiFePO4 batteries. Currently in development and promising alternative technologies would be redox flow batteries. For seasonal storage, although featuring substantially lower cycle efficiencies, hydrogen based systems with regeneration of electrical power by 
internal combustion engines or fuel cells so far are the only potential options. Only when the geography allows it pumped hydro or CAES would be amongst the possibilities, but given the scarcity of such occurrences limited impact is to be expected regarding their contribution.

Up to date, all of the abovementioned technologies, both those commercially available as those in development, have serious shortfalls regarding either their (ecological) sustainability or safety aspects. Batteries require often scarce and/ or highly toxic catalysts for their functioning (examples are nickel and cobalt for lithium intercalation). Moreover, both their electrodes as electrolyte require highly pure carbohydrates as precursors, often exclusively sourced from fossil resources. The extremely high purity requirements for most of these systems to perform as desired also results in high production energy demands, and turns recycling into a tedious business. Hydrogen-based technologies, apart from having an innate low cycle efficiency caused by the anode reaction, often require extremely scarce noble catalysts (e.g. platinum, ruthenium, iridium) for their functioning, resulting in both a high ecological footprint and excessive capital investments required per acquired power capacity. Although in some cases (e.g. a so called "battolyser") nickel(III)oxide-hydroxide may be used for the water splitting reaction, avoiding the use of noble catalysts, this catalyst is not reversible, and thus power can only be regenerated by oxidation of the formed hydrogen (or any of its derivatives) by means of combustion, further lowering the cycle efficiency and adding the complexity of exhaust management ${ }^{[40]}$.

Redox-flow batteries/cells (RFBs), in which the charge carriers/redox active compounds are dissolved/suspended in the electrolytes, and are pumped through the cell stack while the system is operated, have shown promising perspectives. With power density being scalable independently from energy density in these systems, they can be modified to a wide range of uses. A wide variety of redox active compounds have been tested in RFBs so far, most known ones either exploit the various redox states of a transition metal (often vanadium) or combine this to a halogen (often bromine). However, these technologies so far have been pushed back by typically low energy densities ( $<40 \mathrm{Wh} / \mathrm{L})$, safety risks associated to the use of the included metals, low practical energy efficiency due to heating requirements and last but not least: production price. Promising in terms of sustainability and abundance of used materials are recent developments in RFBs using organic redox molecules, most notably (potentially plant-derived) quinones, but up so far these design are hindered by limited solubilities and low cell voltages, leading to insufficient energy density ${ }^{[30]}$. The bioelectrode described in this thesis may prove to be a welcome addition to the existing world of aqueous organic redox flow batteries. With a foreseen charge density for the bioelectrode as high as $185 \mathrm{Ah} \cdot \mathrm{L}^{-1}$, total system energy densities of over $50 \mathrm{Wh} / \mathrm{L}$ may be feasible, once a compatible counter electrode is identified and a fully integrated system is developed. Considering prementioned energy 
capacities required for domestic/small community use (10-1000 kWh) the storage volume required would then be within realistic proportions $\left(0.2-200 \mathrm{~m}^{3}\right)$.

Thus, although the many technical challenges described in this thesis (and possibly more) will need to be solved before allowing up-scale implementation, a carbon dioxide/acetate based AORFB may show to be sustainable, safe and appropriate energy storage technology for decentralized use in the future. 


\subsection{REFERENCES}

(1) He, Z.; Angenent, L. T. T. Application of Bacterial Biocathodes in Microbial Fuel Cells. Electroanalysis 2006, 18 (19-20), 2009-2015.

(2) Huang, L.; Regan, J. M.; Quan, X. Electron Transfer Mechanisms, New Applications, and Performance of Biocathode Microbial Fuel Cells. Bioresour. Technol. 2011, 102 (1), 316-323.

(3) Gong, M.; Dai, H. A Mini Review of NiFe-Based Materials as Highly Active Oxygen Evolution Reaction Electrocatalysts. Nano Res. 2015, 8 (1), 23-39.

(4) Jourdin, L.; Freguia, S.; Donose, B. C.; Chen, J.; Wallace, G. G.; Keller, J.; Flexer, V. A Novel Carbon Nanotube Modified Scaffold as an Efficient Biocathode Material for Improved Microbial Electrosynthesis. J. Mater. Chem. A 2014, 2 (32), 13093-13102.

(5) Marshall, C. W.; Ross, D. E.; Fichot, E. B.; Norman, R. S.; May, H. D. Long-Term Operation of Microbial Electrosynthesis Systems Improves Acetate Production by Autotrophic Microbiomes. Environ. Sci. Technol. 2013, 47 (11), 6023-6029.

(6) Straub, M.; Demler, M.; Weuster-Botz, D.; Dürre, P. Selective Enhancement of Autotrophic Acetate Production with Genetically Modified Acetobacterium Woodii. J. Biotechnol. 2014, 178 (1), 67-72.

(7) Klemps, R.; Schoberth, S. M.; Sahm, H. Production of Acetic Acid by Acetogenium Kivui. Appl. Microbiol. Biotechnol. 1987, 27 (3), 229-234.

(8) Gildemyn, S.; Verbeeck, K.; Slabbinck, R.; Andersen, S. J.; Prévoteau, A.; Rabaey, K. Integrated Production, Extraction, and Concentration of Acetic Acid from CO 2 through Microbial Electrosynthesis. Environ. Sci. Technol. Lett. 2015, 2 (11), 325-328.

(9) Ye, R.; Henkensmeier, D.; Yoon, S. J.; Huang, Z.; Kim, D. K.; Chang, Z.; Kim, S.; Chen, R. Redox Flow Batteries for Energy Storage: A Technology Review. J. Electrochem. Energy Convers. Storage 2017, 15 (1), 010801.

(10) Esteve-Nuñez, A.; Rothermich, M.; Sharma, M.; Lovley, D. R. Growth of Geobacter Sulfurreducens under Nutrient Limiting Conditions in Continuous Culture. Environ. Microbiol. 2005, 7 (5), 641-648.

(11) Raes, S. M. T.; Jourdin, L.; Buisman, C. J. N.; Strik, D. P. B. T. B. Continuous Long-Term Bioelectrochemical Chain Elongation to Butyrate. ChemElectroChem 2017, 4 (2), 386-395.

(12) Ganigué, R.; Puig, S.; Batlle-Vilanova, P.; Balaguer, M. D.; Colprim, J.; Mikkelsen, M.; Jørgensen, M.; Krebs, F. C.; Haszeldine, R. S.; Rabaey, K.; et al. Microbial Electrosynthesis of Butyrate from Carbon Dioxide. Chem. Commun. 2015, 51 (15), 3235-3238.

(13) Jourdin, L.; Raes, S. M. T.; Buisman, C. J. N.; Strik, D. P. B. T. B. Critical Biofilm Growth throughout Unmodified Carbon Felts Allows Continuous Bioelectrochemical Chain Elongation from CO2 up to Caproate at High Current Density. Front. Energy Res. 2018, 6.

(14) Arends, J. B. A.; Patil, S. A.; Roume, H.; Rabaey, K. Continuous Long-Term Electricity-Driven Bioproduction of Carboxylates and Isopropanol from CO2 with a Mixed Microbial Community.J. CO2 Util. 2017, 20, 141-149.

(15) ter Heijne, A.; Schaetzle, O.; Gimenez, S.; Navarro, L.; Hamelers, B.; Fabregat-Santiago, F. Analysis of Bio-Anode Performance through Electrochemical Impedance Spectroscopy. Bioelectrochemistry 2015, 106, 64-72.

(16) Dominguez-benetton, X.; Sevda, S.; Vanbroekhoven, K.; Pant, D. The Accurate Use of Impedance Analysis for the Study of Microbial Electrochemical Systems. Chem. Soc. Rev. 2012, 41, 7228-7246. 
(17) Santoro, C.; Arbizzani, C.; Erable, B.; Ieropoulos, I. Microbial Fuel Cells: From Fundamentals to Applications. A Review. J. Power Sources 2017, 356, 225-244.

(18) Wei, J.; Liang, P.; Cao, X.; Huang, X. A New Insight into Potential Regulation on Growth and Power Generation of Geobacter Sulfurreducens in Microbial Fuel Cells Based on Energy Viewpoint. Environ. Sci. Technol. 2010, 44 (8), 3187-3191.

(19) Hamelers, H. V. M.; Ter Heijne, A.; Stein, N.; Rozendal, R. a; Buisman, C. J. N. Butler-Volmer-Monod Model for Describing Bio-Anode Polarization Curves. Bioresour. Technol. 2011, 102 (1), 381-387.

(20) Aelterman, P.; Freguia, S.; Keller, J.; Verstraete, W.; Rabaey, K. The Anode Potential Regulates Bacterial Activity in Microbial Fuel Cells. Appl. Microbiol. Biotechnol. 2008, 78 (3), 409-418.

(21) González-Muñoz, M.; Dominguez-Benetton, X.; Domínguez-Maldonado, J.; Valdés-Lozano, D.; PachecoCatalán, D.; Ortega-Morales, O.; Alzate-Gaviria, L. Polarization Potential Has No Effect on Maximum Current Density Produced by Halotolerant Bioanodes. Energies 2018, 11 (3), 529.

(22) Baudler, A.; Schmidt, I.; Langner, M.; Greiner, A.; Schröder, U. Does It Have to Be Carbon? Metal Anodes in Microbial Fuel Cells and Related Bioelectrochemical Systems. Energy Environ. Sci. 2015, 8 (7), 2048-2055.

(23) Guo, K.; Prévoteau, A.; Patil, S. A.; Rabaey, K. Engineering Electrodes for Microbial Electrocatalysis. Curr. Opin. Biotechnol. 2015, 33, 149-156.

(24) Kerzenmacher, S. Engineering of Microbial Electrodes. In Advances in Biochemical Engineering/ Biotechnology; Springer, Berlin, Heidelberg, 2017.

(25) Patil, S. A.; Gildemyn, S.; Pant, D.; Zengler, K.; Logan, B. E.; Rabaey, K. A Logical Data Representation Framework for Electricity-Driven Bioproduction Processes. Biotechnol. Adv. 2015, 33 (6), 736-744.

(26) Jourdin, L.; Strik, D. Electrodes for Cathodic Microbial Electrosynthesis Processes : Key-Developments and Criteria for Effective Research \& Implementation. 2017, 2-5.

(27) Tremblay, P.-L.; Angenent, L. T.; Zhang, T. Extracellular Electron Uptake: Among Autotrophs and Mediated by Surfaces. Trends Biotechnol. 2017, 35 (4), 360-371.

(28) Zaybak, Z.; Logan, B. E.; Pisciotta, J. M. Electrotrophic Activity and Electrosynthetic Acetate Production by Desulfobacterium Autotrophicum HRM2. Bioelectrochemistry 2018, 123, 150-155.

(29) Liu, T.; Wei, X.; Nie, Z.; Sprenkle, V.; Wang, W. A Total Organic Aqueous Redox Flow Battery Employing a Low Cost and Sustainable Methyl Viologen Anolyte and 4-HO-TEMPO Catholyte. Adv. Energy Mater. 2016, 6 (3).

(30) Hu, B.; DeBruler, C.; Rhodes, Z.; Liu, T. L. Long-Cycling Aqueous Organic Redox Flow Battery (AORFB) toward Sustainable and Safe Energy Storage. J. Am. Chem. Soc. 2017, 139 (3), 1207-1214.

(31) Desmond-Le Quéméner, E.; Bouchez, T. A Thermodynamic Theory of Microbial Growth. ISME J. 2014, 8 (8), 1747-1751.

(32) Sleutels, T. H. J. A.; Darus, L.; Hamelers, H. V. M.; Buisman, C. J. N. Effect of Operational Parameters on Coulombic Efficiency in Bioelectrochemical Systems. Bioresour. Technol. 2011, 102 (24), 11172-11176.

(33) Jourdin, L.; Freguia, S.; Flexer, V.; Keller, J. Bringing High-Rate, CO2-Based Microbial Electrosynthesis Closer to Practical Implementation through Improved Electrode Design and Operating Conditions. Environ. Sci. Technol. 2016, 50 (4), 1982-1989.

(34) Bouwer, E. J.; McCarty, P. L. Effects of 2-Bromoethanesulfonic Acid and 2- Chloroethanesulfonic Acid on Acetate Utilization in a Continuous-Flow Methanogenic Fixed-Film Column. Appl. Envir. Microbiol. 1983, 45 (4), 1408-1410. 
(35) Belay, N.; Daniels, L. Production of Ethane, Ethylene, and Acetylene from Halogenated Hydrocarbons by Methanogenic Bacteria. Appl. Environ. Microbiol. 1987, 53 (7), 1604-1610.

(36) Maria-Magdalena Titiric; Robin J. White; Brun, N.; Budarin, V. L.; Su, D. S.; Monte, F. del; Clark, J. H.; MacLachlan, M. J. Sustainable Carbon Materials. Chem. Soc. Rev. 2015, 44, 250-290.

(37) Sorokin, D. Y.; Tourova, T. P.; Muyzer, G. Isolation and Characterization of Two Novel Alkalitolerant Sulfidogens from a Thiopaq Bioreactor, Desulfonatronum Alkalitolerans Sp. Nov., and Sulfurospirillum Alkalitolerans Sp. Nov. Extremophiles 2013, 17 (3), 535-543.

(38) Mateos, R.; Sotres, A.; Alonso, R. M.; Escapa, A.; Morán, A. Impact of the Start-up Process on the Microbial Communities in Biocathodes for Electrosynthesis. Bioelectrochemistry 2018, 121, $27-37$.

(39) Tomai, T.; Saito, H.; Honma, I. High-Energy-Density Electrochemical Flow Capacitors Containing Quinone Derivatives Impregnated in Nanoporous Carbon Beads. J. Mater. Chem. A 2017, 5 (5), 21882194.

(40) Mulder, F. M.; Weninger, B. M. H.; Middelkoop, J.; Ooms, F. G. B.; Scheuders, H. Efficient Electricity Storage with a Battolyser, an Integrated Ni-Fe Battery and Electrolyser. Energy Environ. Sci. 2017, No. 3, 756-764.

(41) Grattieri, M.; Minteer, S. D. Microbial Fuel Cells in Saline and Hypersaline Environments: Advancements, Challenges and Future Perspectives. Bioelectrochemistry 2018, 120, 127-137.

(42) Sun, D.; Call, D.; Wang, A.; Cheng, S.; Logan, B. E. Geobacter Sp. SD-1 with Enhanced Electrochemical Activity in High-Salt Concentration Solutions. Environ. Microbiol. Rep. 2014, 6 (6), 723-729. 


\subsection{APPENDIX: PRACTICAL EXPLORATION OF CHOICES IN MASS TRANSFER CHARACTERISTICS BY CELL DESIGN}

In section 7.2, three prime design considerations were identified which have a defining impact on mass transfer characteristics of the MRB. To further illustrate what such an integrated approach could entail, Table $\mathbf{S 1}$ lists all combinations that can be made using the three mentioned foremost design choices (membrane type, buffer type, counter reaction type). For this illustration, membrane typology has been defined on basis of charge selectivity; and as such, four options, being AEM, CEM or a combination of these (AEM-CEM or CEM-AEM), have been taken into consideration.

Subsequently, for each of the combinations listed in Table S1, a corresponding schematic figure, with major mass transfer flows as expected to occur indicated, has been made. These can be found in the appendix, under the label as indicated in the table (Figure S1-16). It should be explicitly mentioned these schematics, and the processes indicated, by no means aim to provide completeness or physical accuracy, rather, they serve the point of illustration and thought-experiment.

Taking into consideration the various options as they arise from Table S1 and as they are depicted in Figures \$1-16, the next logical question then becomes: which one is the best? Here it becomes tricky, as in making a qualitative comparison of these conceptual scenarios, practical considerations have to be made with regard to the specific risks as they either do or do not apply to each option.

TABLE S1 | Cross-table of all possible cell designs based on combinations of buffer type (volatile or non-volatile), counter electrode reaction type (proton coupled or non-proton coupled) and applied membrane (anion exchange, cation exchange, or bipolar/combined AEM/CEM). Number annotations of combinations (S1-S16) refer to figure numbers in which the designs are schematically depicted.

\begin{tabular}{|c|c|c|c|c|c|c|c|c|}
\hline \multirow[b]{3}{*}{ 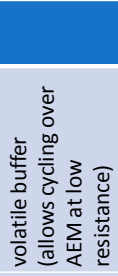 } & \multicolumn{4}{|c|}{ PCET (potentially partially buffered) } & \multicolumn{4}{|c|}{$\begin{array}{l}\text { NPCET (requires full buffering on anode side, salt } \\
\text { required at cathode) }\end{array}$} \\
\hline & AEM & CEM & AEM-CEM & CEM-AEM & AEM & CEM & AEM-CEM & CEM-AEM \\
\hline & S1 & S3 & S5 & S7 & S9 & S11 & S13 & S15 \\
\hline 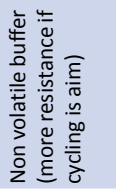 & S2 & S4 & S6 & S8 & S10 & S12 & S14 & S16 \\
\hline
\end{tabular}


In making this "risk assessment", the main factors that pose a risk to full-cell performance can be listed as follows:

- Coulombic efficiency compromising factors:

- crossover issues: requirements regarding membrane selectivity, or, alternatively, requirements regarding the nature of the counter reactant (e.g. immobilization, state of charge). A particularly tricky factor here may be water transfer over the membrane in case large osmotic differences between compartments exist: this may only be compensated by RO, the high operation pressures of which would most likely compromise cell design.

- Voltage efficiency compromising factors (rate limiting / entropy producing processes):

- Membrane resistance: less membranes and less selective membranes in general yields lower resistance, but can be in conflict with high CE

- Scaling tendencies: working with higher salinity is tricky, as this may result in clogging of membranes or electrodes. Even if precipitation is part of the scheme and well controlled (e.g. by strategically placed nucleation sites), low dissolution rates and (re)hydration energy may induce substantial energy losses, or limit current densities severely

- Gas storage: to some extent inevitably for excess $\mathrm{CO}_{2}$ as it is present in discharged state, but especially in case a volatile buffer is used in combination with a design that does not allow for proton shuttling between anode and cathode, gas will need to be stored and returned to the electrolyte throughout charging/discharging cycles. This will require compression energy, and the vaporization and dissolution will induce entropy losses in the form of hydration energy, all negatively affecting energy efficiency.

- Biocompatibility compromising factors: without active bacteria, the MRB will simply not work. Depending on the used inoculum / bacterial communities, and their tolerance towards changes in/levels of $\mathrm{pH}$ and salinity, controlling these variables may or may not play an important role in cell design. However by default, neutral pH and salinities up to $35 \mathrm{~g} / \mathrm{L}$ (marine conditions) may be taken as safe points of departure. (Large) deviations from this will most likely require the identification and application of extremophilic communities, for which less knowledge is currently available regarding their application in BESs.

- Energy density compromising factors: Aside from the charge density of the counter electrode reaction, the obtainable energy density is depending on additional operational volumes required for monitoring and control. Although some parts, like electronics and pumps, need to be present regardless system design, there are two storage volumes which only need to be present in certain specific configurations, and in that case may take up significant amount of space, negatively affecting energy density: 
- Middle compartment: in case the counter reactant is positively charged and mobile, and no membrane can be found which is selective (enough) on basis of steric hindrance, at least two membranes will have to be applied in order to keep oxidant and reductant apart. A most logical approach is then to use the concept of electrodialysis, with a middle compartment where salt/buffer is accumulated/ depleted from throughout charging/discharging (e.g. Figure S15). Although such designs may show to be advantageous regarding $\mathrm{CE}$ and biocompatibility, the middle compartment and its contents will take up space as well, lowering energy density of the technology.

- Gas storage: apart from inducing some energy losses by means of hydration and compression energy, storing gas requires volume (extent which is depending on compression factor). This lowers energy density as well.

In Table S2, the above list of potential risks is applied to the options from Table S1, to obtain a qualitative comparison between cell designs.

Looking at Table S2, it should be noted that a quantitative assessment of the mentioned criteria is almost impossible. Therefore, it is hard - if not impossible to say aforehand which of the assessed options would be a more successful / better performing battery. To illustrate this point, as relevant questions may serve to what extent the inoculum can withstand high salinity and / or fluctuations thereof, or what is known about the $\mathrm{pH}$ range in which bacteria remain active. If strict operational limits apply to these ends, biocompatibility criteria will weigh in considerably more than when known extremophiles are at hand.

Following this line for the sake of demonstration, if for now we assume salinity and $\mathrm{pH}$ tolerances to be restricted by the range as applied in Straub's study ${ }^{[6]}$, there is a focus on achieving biological compatibility as such. In that case, two cell designs stand out, being Figures S1 and S13. These two options, together with Figure S9, seem most suitable for achieving constant and low salinity and neutral $\mathrm{pH}$ at the anode. However, these options are scoring less promising on other criteria: e.g. the requirement of $\mathrm{CO}_{2}$ addition (charging) and stripping (discharging), would pose an additional complexity to the system as it requires a $\mathrm{CO}_{2}$ storage and dosing system. If studies show however that high salt tolerance can be combined with good bacterial activity, then these criteria would be weighed differently and preferred choice could shift to (one of the) other concepts as outlined in the series of figures S1-16.

Due to the foreseen advantages of increased pH buffering and salinity on electrolyte conductivity, the research in halophilic and halotolerant species for BES applications has caught more interest recently ${ }^{[41]}$. For the bioanode, first reports 


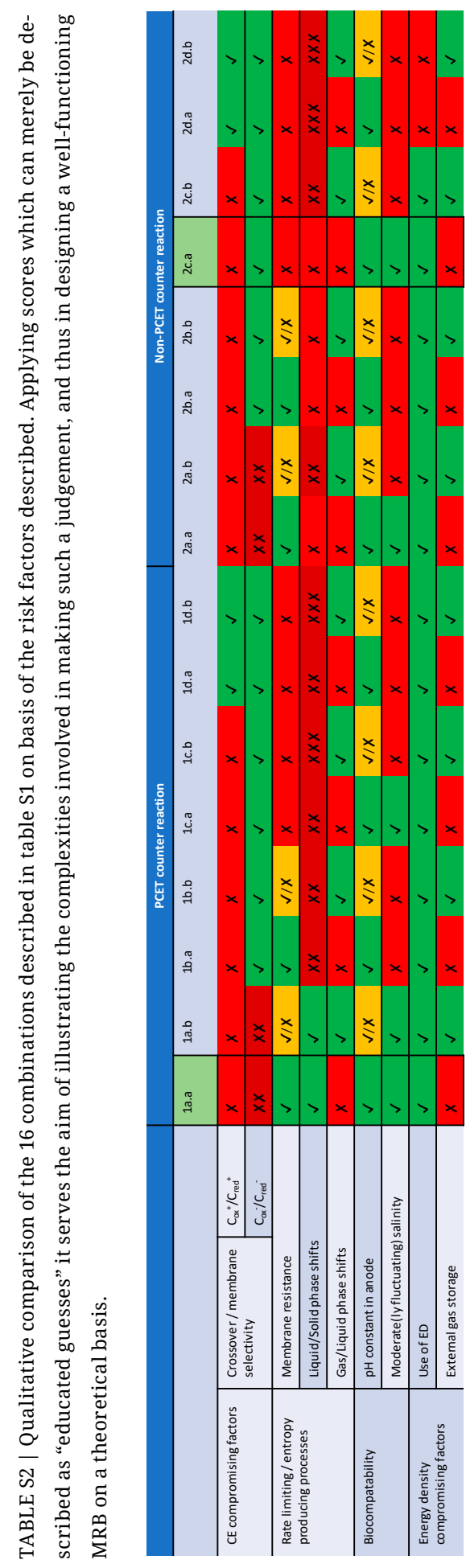


show excellent performance of some electrogenic species for acetate oxidation at salt concentrations around $0.6 \mathrm{M}^{[42]}$. Regarding $\mathrm{CO}_{2}$ reducing biocathodes, less is known about potential tolerance to salt. However, apart from the appreciable salt concentrations used in the works presented in this thesis (around $0.3 \mathrm{M}$ ), the occurrence and activity of acetogens in soda lakes is ubiquitous. In such lakes, salt concentrations typically fluctuate throughout the seasons, varying as extreme as from saturation point to estuarine conditions in some cases. Taking this into account, although challenging, this provides an optimistic outlook for finding suitable microbes, catalyzing the bidirectional electrode, that can tolerate such a highly saline and fluctuating environment.

In conclusion, it is clear that for increasing acetate concentrations for this electrode, while not being compromised in terms of VE/CE and thus EE, or stability, multiple design aspects will have to be considered altogether in order to find a suitable compromise between various pros and cons. As was also concluded in the discussion of this thesis, given the complexity and versality of this task, a long road of experimenting presumably still lies ahead. 
\$1.

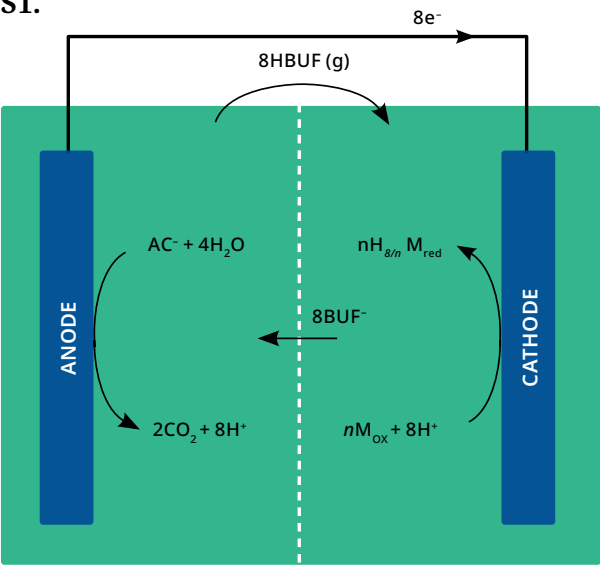

AEM

S3.

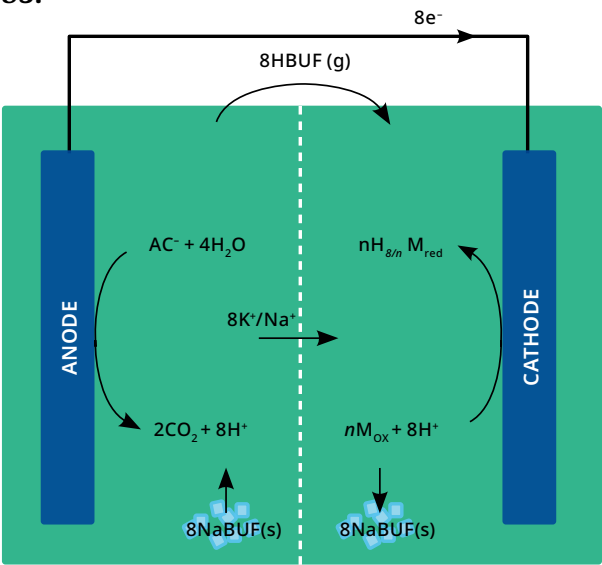

CEM

S5.

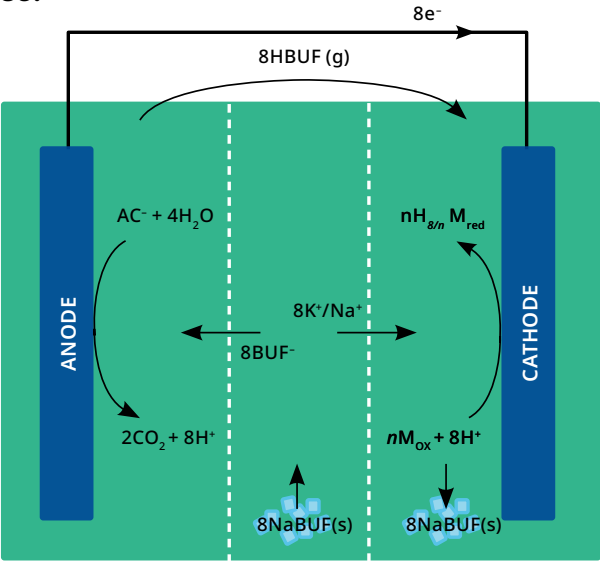

AEM

CEM
S2.

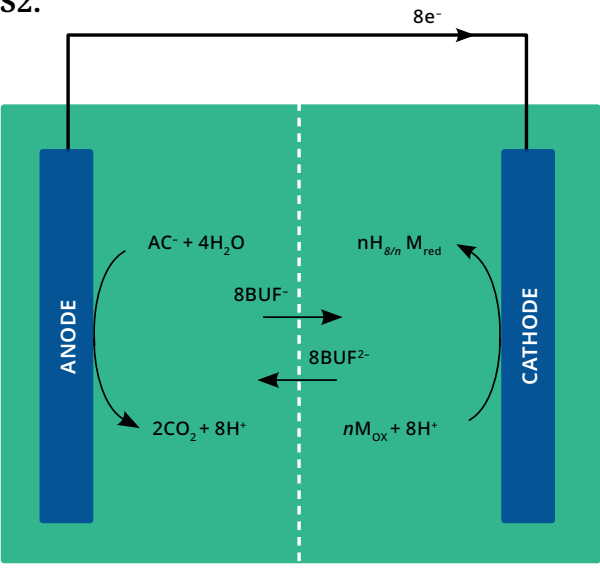

AEM

S4.

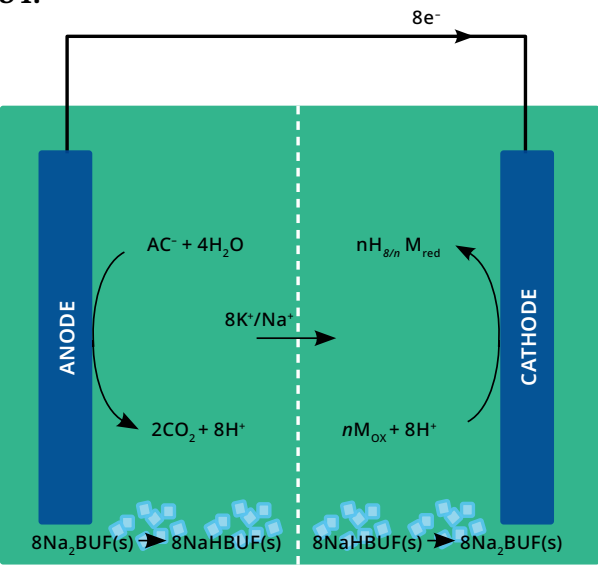

CEM

S6.

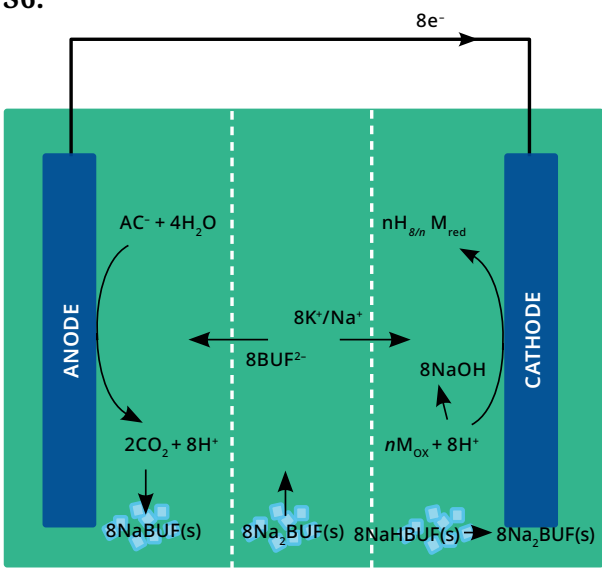

AEM 
S7.

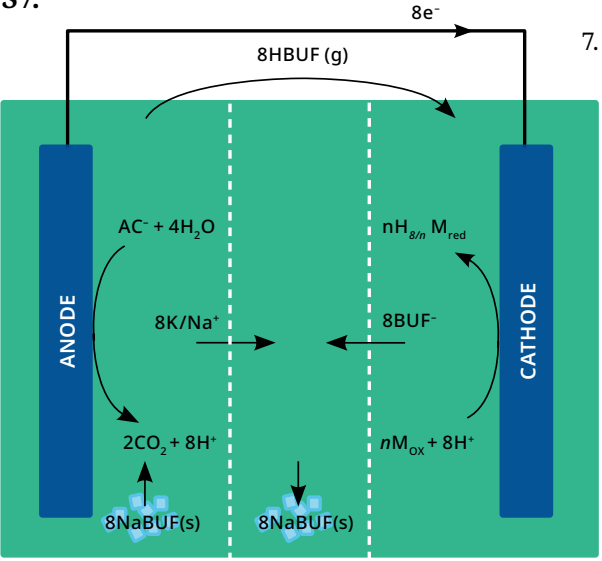

s9.

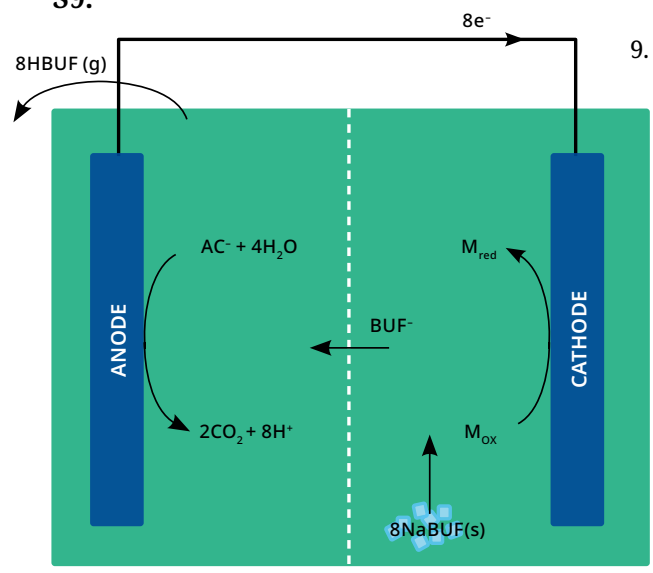

AEM

S11.

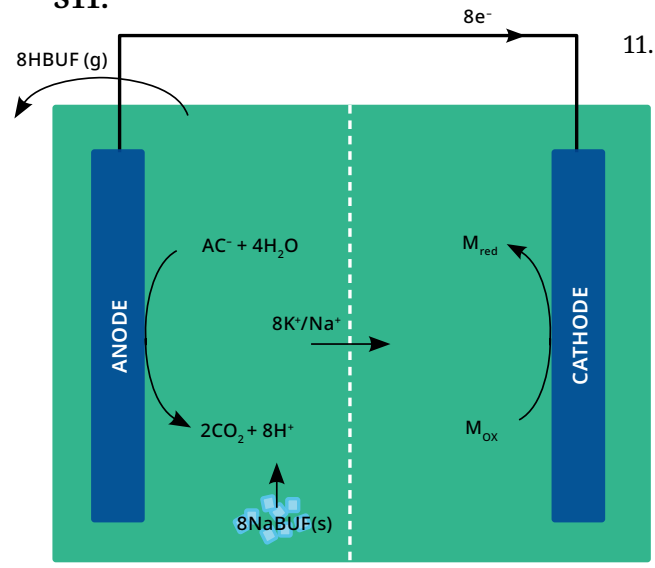

CEM s8.

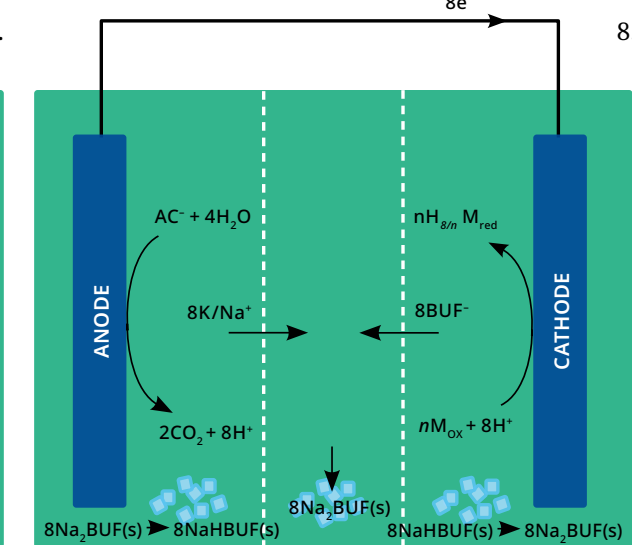

$8 \mathrm{Na}_{2} \mathrm{BUF}(\mathrm{s})>8 \mathrm{NaHBUF}(\mathrm{s})$

CEM AEM

S10.

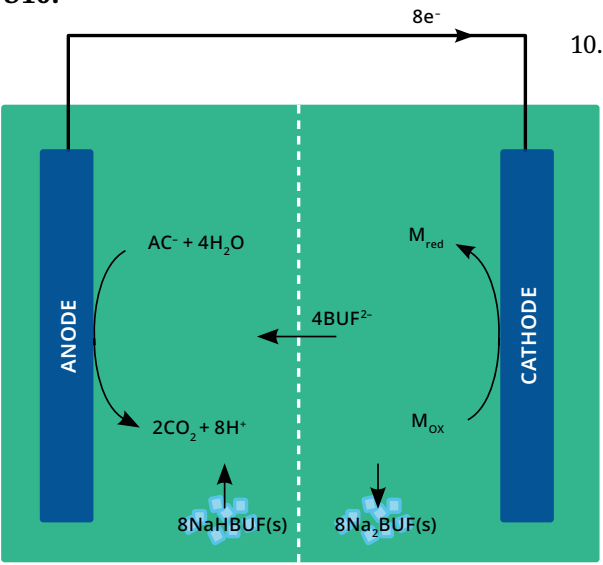

10

S12.

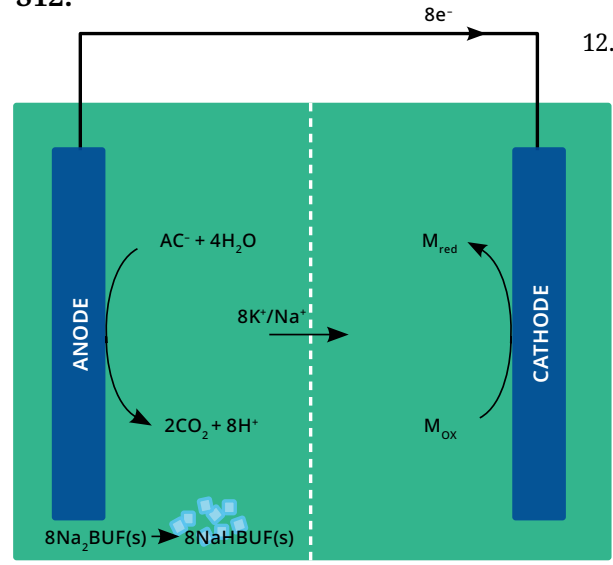

CEM 
S13.

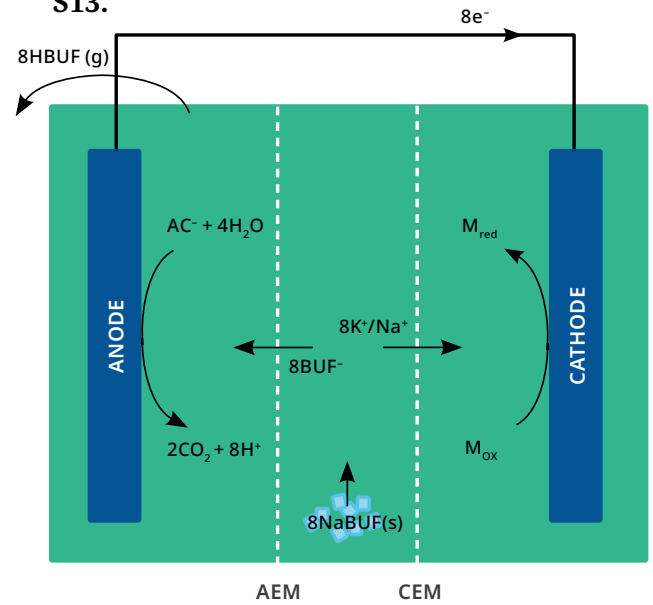

S13.

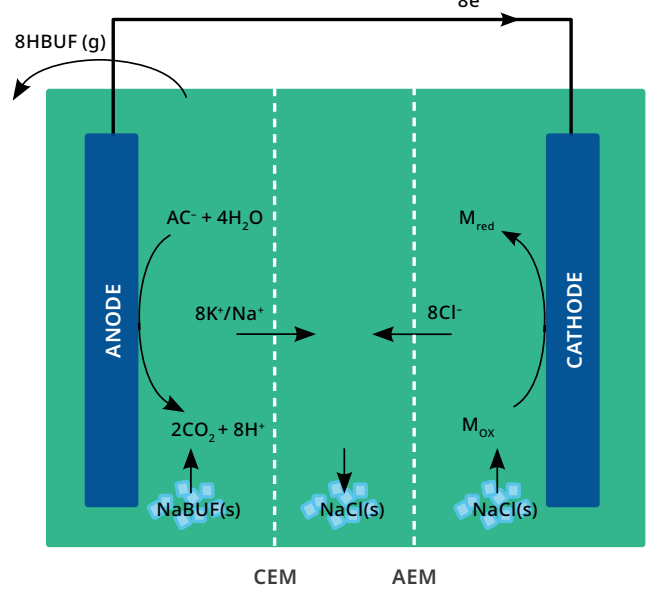

S14.

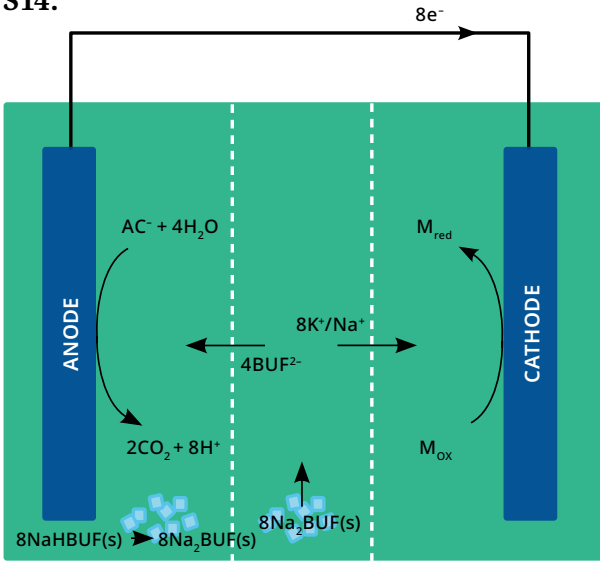

AEM

CEM

S14.

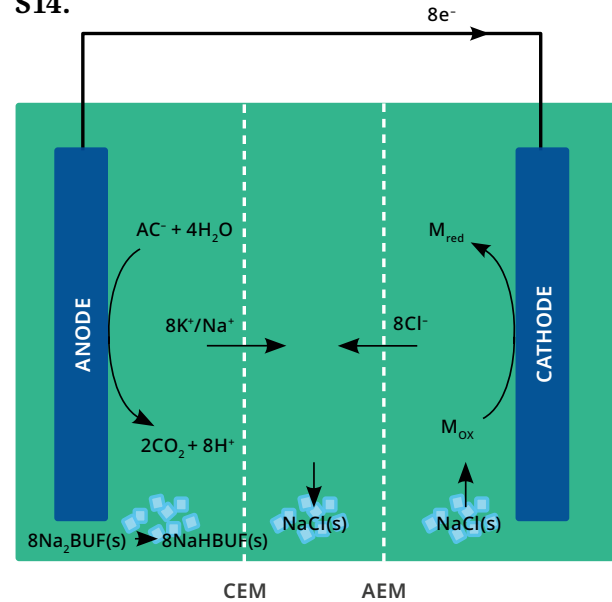

FIGURE S1-S16 | Schematic drawings of electrochemical cells based on the combinations made in Table S1, varying in (a) applied buffer system, (b) type of membranes used, (c) type of counter electrode reaction. Indicated are the major anticipated electrolytic mass and charge transfer flows, which may be accommodated, depending on the exact figure, by (i) volatile buffer system HBUF/BUF;, (ii) non-volatile buffer system BUF-/BUF ${ }^{2}$, (iii) potassium or sodium cations $\left(\mathrm{K} / \mathrm{Na}^{+}\right)$. Whenever accumulation/depletion of both cations and anions in a compartment is inherent to the process, the respective salts likely to deposit/dissolve are indicated, these may be in the case of a volatile buffer system the sodium/potassium salts of the monovalent anions (indicated as NaHBUF), while for the non-volatile/divalent buffer this may be the sodium/potassium salts of both monovalent and divalent anions (indicated as NaHBUF and $\mathrm{Na}_{2} \mathrm{BUF}$ ). In case a volatile buffer is applied, gaseous removal of the protonated buffer (HBUF) may be applied to control $\mathrm{pH}$, at least theoretically. 



\section{SUMMARY}




\section{Microbially catalyzed electrochemical systems for sustainable energy storage}

A smooth and complete transition towards renewable energy is challenged by the variability of generation of power from renewable resources, which cannot by themselves function as baseload generation. Under business-as-usual scenarios, the integration of renewables beyond $30 \%$ of total grid power generation is generally expected to require additional measures to guarantee baseload providence. Energy Storage Technologies (ESTs) which store variable generated renewable energy could effectively replace the current fossil fuel driven baseload generation, and are therefore widely seen as potential key players in the renewable energy transition. However, in anticipation of such an intensified future role for energy storage technologies, it becomes increasingly important for the production, operation and recommissioning of these storage facilities not to be in conflict with the sustainability endeavors which their intended use is part of. In this thesis, the use of Bioelectrochemical Systems (BESs) for energy storage is proposed as a possible technology to address this challenge.

As is described in more detail in Chapter 1, microorganisms in BESs are able to sustain their growth and activity by using electrodes either as the electron acceptor or donor required for driving their cellular respiration. Microorganisms are able to do so by exploiting various mechanisms, transferring electrons between an electrode and the microbial cell. This way, a wide variety of complex electron transfer chains (ETCS) naturally present in microbial communities, carrying out the redox reactions occurring in microbial catabolism, from oxidation of a wide range of carbohydrates, ammonium and fatty acids to the reduction of nitrate, oxygen, carbon dioxide or sulphate, may be ported to the artificial environment of a man-made electrochemical cell.

Bioelectrochemical systems (BES) hold potential both for conversion of electricity into chemicals through microbial electrosynthesis (MES), and the provision of electrical power by oxidation of organics using microbial fuel cells (MFC) and have been intensively studied with regard to these two separate applications. However, when the works covered in this thesis commenced, combining MES and MFC in a single system had not been done before, while obviously this would hold the premise of providing a power-to-power electrical storage technology. It was hypothesized that, by combining MES and MFC, microbially catalysed electrical energy storage may be possible, based on abundant, low-cost and environmentally-friendly chemicals.

Chapter 2 of this thesis provides a proof-of-concept for a Microbial Rechargeable Battery (MRB) allowing storage of electricity by combining MES and a MFC in one system. For this first proof, hexacyanoferrate (II/III) was used as counter redox couple. Duplicate runs showed stable performance during 15 days, with acetate being the main energy carrier. An energy density of around 
$0.1 \mathrm{kWh} / \mathrm{m}^{3}$ (normalized to anode electrolyte volume) was achieved at full-cycle energy efficiency of 30 to $40 \%$, with a nominal power output during discharge of $190 \mathrm{~W} / \mathrm{m}^{3}$ (normalized to anode volume).

\section{Prevention of methanogenesis in bioanodes}

A crucial aspect for a well-performing MRB, and for bioelectrochemical systems (BESs) in general, is the efficient oxidation of substrates by the bioanode, which is reflected in high Coulombic efficiency (CE). In bioanodes, the formation of methane from more reduced substrates impedes CE as these substrates are then no longer available for production of electrical current. As such, methanogenesis forms a major threat to $\mathrm{CE}$ in BESs, while core to the field of conventional anaerobic digestion (AD), and much wanted in methane producing biocathodes for power-to-gas technologies. To obtain high CEs and to prevent the formation of methane in the MRB, in the proof-of-concept the halogenated organic compound 2-bromoethanosulfonate (2-BES) was added as a known selective inhibitor for production of methane. However, the sustainability of this practice, both from environmental and technical perspective, may be disputed, as is discussed with more detail in Chapter 7.

Instead of using (2-BES) to prevent methane formation, in Chapter 3 alternative approaches are explored to achieve high bioanode $\mathrm{CE}$, by providing a competitive advantage to electrogens over methanogens. Factors identified that affect this competition in bioanodes are, amongst others, electrolyte composition (most notably substrate concentration, conductivity and $\mathrm{pH}$ ) and anode potential. Focus is put on acetate as a substrate and the competition between methanogens and electrogens is analyzed from a thermodynamic and kinetic point of view. Based on a review of experimental data from earlier studies, it is proposed that low substrate loading in combination with a sufficiently high anode overpotential results in favorable growth kinetics of electrogens compared to methanogens. Thus, anode potential and substrate loading rate may be used as operational parameters for controlling $\mathrm{CE}$ at desirable levels, especially in open systems, or in semi-open systems where microbial composition cannot be adequately controlled for. To achieve high current density in combination with low substrate concentrations, it is essential to have a high specific anode surface area. New reactor designs with these features are essential for BESs to be successful in wastewater treatment in the future. As is discussed in more detail in chapter 7, the possible implications of the developed theory for the MRB would be that instead of using 2-BES for methane inhibition during discharging, a redox flow cell-like configuration could be adopted in which the bioanode gradually receives acetate at a loading rate ensuring proper CE development, while current density is modulated to optimize anode potential during discharge. 


\section{Prevention of methanogenesis in biocathodes}

As a logical follow up to the work done on CE in bioanodes, Chapter 4 focuses on operational strategies for biocathodes used in Microbial electrosynthesis (MES). Apart from being indispensable to the concept of the MRB, MES is a useful technology for the renewable production of organic commodities from biologically catalyzed reduction of $\mathrm{CO}_{2}$. However, for the technology to become applicable, process selectivity, stability and efficiency needs further improvements. The effects were analysed of different electrochemical control modes (potentiostatic/ galvanostatic) on both the start-up characteristics and steady-state performance of biocathodes using a non-enriched mixed culture inoculum. Based on results obtained, it is concluded that kinetic differences exist between the two dominant functional microbial groups (i.e. homoacetogens and methanogens) and that by applying different current densities, these differences can be exploited to steer product selectivity and reactor performance. This is due mainly to the observation that at high hydrogen partial pressures, acetogens may outgrow methanogens. We pose that, through future optimizations by these means, acetate producing MES can be sustained at high production rates and product selectivity without the need of methanogen-inhibiting chemical additives or pre-enrichment of inoculum, albeit at the (slight) cost of voltage efficiency. As is discussed more extensively in chapter 7, this theory could be implemented to the concept of the MRB - in case required - by increased current densities upon charging, while keeping solid retention time low.

\section{Studying microbial growth dynamics in BES: looking at the biofilm}

Where chapters 3 and 4 established qualitative relationships between operational parameters and system performance indicators, a broadly shared demand for quantitative data exists within BES research. Chapter 5 stresses the need of studying in more detail the microbial growth dynamics in bioelectrochemical systems, in order to allow for their proper design and operation. To address this need, Optical Coherence Tomography (OCT) was applied as a tool for in situ and non-invasive quantification of biofilm growth on electrodes (bioanodes). An experimental platform is designed and described in which transparent electrodes are used to allow for real-time, three-dimensional biofilm imaging. The accuracy and precision of the developed method is assessed by relating OCT results to well-established standards for biofilm quantification (COD and Total N) and show high correspondence to these standards. Biofilm thickness as observed by OCT ranged between 3 and $90 \mu \mathrm{m}$ for experimental durations ranging from 1 to 24 days. This translated to growth yields between 38 and $42 \mathrm{mg} \mathrm{COD}$ biomass $/ \mathrm{g}$ 
$\mathrm{COD}_{\text {acetate }}$ at an anode potential of $-0.35 \mathrm{~V}$ vs. $\mathrm{Ag} / \mathrm{AgCl}$. Time-lapse observations of an experimental run performed in duplicate show high reproducibility in obtained microbial growth yield using the developed method. We identify OCT as a powerful tool for conducting in-depth characterizations of microbial growth dynamics in BESs. Additionally, the presented platform allows concomitant application of this method with various optical and electrochemical techniques.

\section{Making the MRB more sustainable: two cathodes put to the test}

The proof-of-concept described in Chapter 1 shows that bioelectrochemical $\mathrm{CO}_{2}$ reduction and subsequent product oxidation may successfully be combined in one integrated system. However, the ferricyanide/ferrocyanide counter electrode used in this first experiments proofed unstable under the conditions tested after prolonged testing periods. For further development of the MRB, a suitable alternative counter electrode needs to be found. In Chapter 6 , two alternative counter electrodes types are put to the test - namely (i) oxygen/water and (ii) a capacitive electrode - for use in the MRB platform. During daily charge/discharge cycling over periods of 11 to 15 days, experimentally obtained energy efficiencies of 25 and $3.7 \%$ were reported when using the capacitive and the oxygen/water electrodes, respectively. Large overpotentials, resulting in a voltage efficiency of $15 \%$ and oxygen crossover leading to Coulombic efficiencies of $25 \%$ caused the considerably lower efficiency for the oxygen/water systems, despite the theoretical higher voltage efficiency. Although the capacitive electrode equipped systems performed better, energy density is limited by the operational potential window within which capacitive systems can operate reliably.

\section{Go/No Go? A comparative analysis of the MRB and guide to future designs}

Despite the disappointing results regarding so-far tested counter electrodes, in Chapter 7, the microbially catalyzed carbon dioxide/acetate redox couple is identified as a promising anode for future implementation in Aqueous Organic Redox Flow Batteries (AORFB). It is concluded that the bidirectional carbon dioxide/ acetate bioanode may be competitive to, or even outperforms most currently investigated redox chemistries for AORFBs. The bioelectrode described in this thesis may prove to be a welcome addition to the existing world of aqueous organic redox flow batteries. That said, the impracticalities encountered so far with tested counter electrodes underline the importance of accounting for charge transport and separation of compounds between anode and cathode in system design, and as 
most important design considerations are identified (a) membrane type, (b) buffer selection and (c) proton coupled electron transfer at the counter electrode reaction.

It is emphasized that, based partially on standing practice in hydrogenotrophic fermentation, charge density for the bioelectrode as high as $185 \mathrm{Ah} \cdot \mathrm{L}^{-1}$ may be reached. This would translate to total system energy densities of over $50 \mathrm{Wh} / \mathrm{L}$ to be feasible, once a compatible counter electrode is identified and a fully integrated system is developed. Considering energy capacities required for domestic/small community use (10-1000 $\mathrm{kWh}$ ) the storage volume required would then be within realistic proportions $\left(0.2-200 \mathrm{~m}^{3}\right)$.

Provided the many reservations made throughout this thesis, a carbon dioxide/ acetate based AORFB may show to be sustainable, safe and appropriate energy storage technology for decentralized use in the future. 



\section{SENSE}

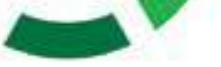

Netherlands Research School for the

Socio-Economic and Natural Sciences of the Environment

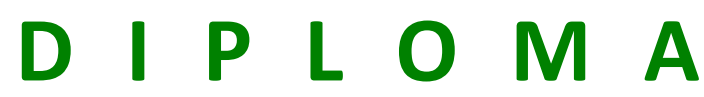

For specialised PhD training

The Netherlands Research School for the Socio-Economic and Natural Sciences of the Environment (SENSE) declares that

\section{Sam Daniël Molenaar}

born on 24 April 1986 in Amsterdam, The Netherlands

has successfully fulfilled all requirements of the Educational Programme of SENSE.

Wageningen, 7 November 2018

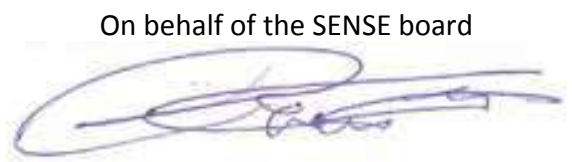

Prof. dr. Huub Rijnaarts the SENSE Director of Education

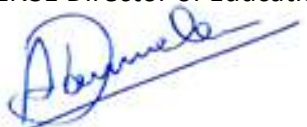

Dr. Ad van Dommelen

The SENSE Research School has been accredited by the Royal Netherlands Academy of Arts and Sciences (KNAW)

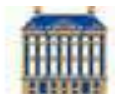




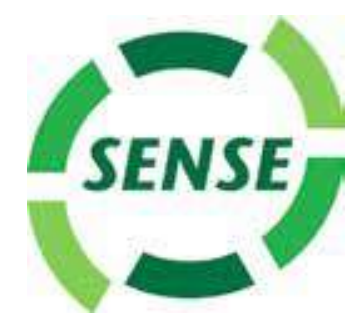

The SENSE Research School declares that Sam Daniël Molenaar has successfully fulfilled all requirements of the Educational PhD Programme of SENSE with a work load of $61.5 \mathrm{EC}$, including the following activities:

\section{SENSE PhD Courses}

- Environmental research in context (2012)

- Research in context activity: 'Developing and organising a workshop during the European Summer School of Electrochemical Engineering (ESSEE) in Leeuwarden' (2015)

\section{Other PhD and Advanced MSc Courses}

- Mathematical principles, Wetsus Academy (2011)

- Chemical reactor design, Wetsus Academy (2011)

- Multicomponent mass transfer in membrane processes, Wetsus Academy (2011)

- Transport phenomena in water technology, Wetsus Academy (2011)

- Colloid chemistry, Wetsus Academy (2011)

- Biological wastewater treatment and recovery technology, Wetsus Academy (2011)

- Supervision of thesis students by PhD researchers, Wetsus (2014)

- European summer school of electochemical engineering, Leeuwarden (2015)

- Project and time management, Wageningen Graduate Schools (2017)

- Career orientation course, Wageningen Graduate Schools (2017)

\section{External training at a foreign research institute}

o Electrochemical sensor development, Endress+ Hauser, Waldheim, Germany (2015)

\section{Management and Didactic Skills Training}

- Supervising 7 MSc students and 2 BSc students with thesis (2014-2017)

- Organizer of ESEE Summer School 2015, Wetsus, Leeuwarden

\section{Oral Presentations}

- Competition between electrogens and methanogens in BESs. Mini-symposium at Cawthron Institute /Massey University, 17-21 February 2014, Nelson/ Palmerston North, New Zealand

- Competition between electrogens and methanogens in BESs. Mini-symposium at Endress Hauser 15 April 2015, Waldhiem, Germany

- Microbial Rechargeable Battery. Mini-symposium at Liandon, 20 May 2016, Duiven, The Netherlands

- Microbial Rechargeable Battery. Award winning poster presentation and technology demonstration at the International Symposium for Microbial Electrochemical Technologies (ISMET), 2016, Rome

SENSE Coordinator PhD Education

Dr. Peter Vermeulen 



\section{Propositions}

1. The bidirectional carbon dioxide/acetate bioanode features excellent propertiesforapplicationwithinaqueousorganicredoxflowbatteries. (this thesis)

2. Methanogenesis poses a major risk to system performance in long-term operation of anaerobic bioelectrochemical systems. (this thesis)

3. In modern agriculture, the importance of maintaining ecosystem integrity as a necessity for long term stability, is structurally overlooked.

4. Instead of accommodating an anticipated increase in human population size, science and technology should aim to accommodate a dignified decline thereof.

5. Truth and freedom are mutually exclusive, making the human pursuit of possessing both, absurd.

6. The role of science and technology in our modern western society, exhibits many features normally associated with religion.

Propositions belonging to the thesis, entitled

'Optimization Of Bioelectrochemical Systems

For Sustainable Energy Storage'

Sam Molenaar

Wageningen, 7 November, 2018 
The research described in this thesis was financially supported by The Dutch Financer.

Financial support from both Wageningen University and Wetsus for printing this thesis is gratefully acknowledged. 
Cover design by Lisa Droës Interior design by Lyanne Tonk 
